\title{
STUDY OF CARRIER INJECTION FOR PERFORMANCE OPTIMIZATION OF A RECONFIGURABLE WAVEGUIDE DIGITAL OPTICAL SWITCH ON InGaAsP/InP
}

by

\author{
Sandy Ng, B.A.Sc., M.A.Sc. \\ A thesis submitted to the Faculty of Graduate Studies and Research \\ in partial fulfillment of the requirements for the degree of \\ Doctor of Philosophy \\ Ottawa-Carleton Institute for Electrical and Computer Engineering \\ Department of Electronics \\ Faculty of Engineering \\ Carleton University \\ Ottawa, Ontario, Canada
}

Copyright $\odot 2006$ by Sandy Ng 


$\begin{array}{ll}\begin{array}{l}\text { Library and } \\ \text { Archives Canada }\end{array} & \begin{array}{l}\text { Bibliothèque et } \\ \text { Archives Canada }\end{array} \\ \begin{array}{l}\text { Published Heritage } \\ \text { Branch }\end{array} & \begin{array}{l}\text { Direction du } \\ \text { Patrimoine de l'édition }\end{array} \\ \begin{array}{l}\text { 395 Wellington Street } \\ \text { Ottawa ON K1A ON4 }\end{array} & \begin{array}{l}\text { 395, rue Wellington } \\ \text { Ottawa ON K1A ON4 } \\ \text { Canada }\end{array}\end{array}$

Your file Votre référence ISBN: 978-0-494-23296-5 Our file Notre référence ISBN: 978-0-494-23296-5

NOTICE:

The author has granted a nonexclusive license allowing Library and Archives Canada to reproduce, publish, archive, preserve, conserve, communicate to the public by telecommunication or on the Internet, loan, distribute and sell theses worldwide, for commercial or noncommercial purposes, in microform, paper, electronic and/or any other formats.

The author retains copyright ownership and moral rights in this thesis. Neither the thesis nor substantial extracts from it may be printed or otherwise reproduced without the author's permission.
AVIS:

L'auteur a accordé une licence non exclusive permettant à la Bibliothèque et Archives Canada de reproduire, publier, archiver, sauvegarder, conserver, transmettre au public par télécommunication ou par l'Internet, prêter, distribuer et vendre des thèses partout dans le monde, à des fins commerciales ou autres, sur support microforme, papier, électronique et/ou autres formats.

L'auteur conserve la propriété du droit d'auteur et des droits moraux qui protège cette thèse. $\mathrm{Ni}$ la thèse ni des extraits substantiels de celle-ci ne doivent être imprimés ou autrement reproduits sans son autorisation.
In compliance with the Canadian

Privacy Act some supporting forms may have been removed from this thesis.

While these forms may be included in the document page count, their removal does not represent any loss of content from the thesis.
Conformément à la loi canadienne sur la protection de la vie privée, quelques formulaires secondaires ont été enlevés de cette thèse.

Bien que ces formulaires aient inclus dans la pagination, il n'y aura aucun contenu manquant.

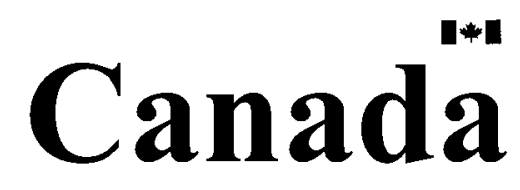




\section{Abstract}

This thesis presents experimental data on the optical performance of InGaAsP/InP active devices, including digital optical switches (DOSs) and bend-loss modulators, actuated by carrier injection as well as the electro-optical effect. It is demonstrated that carrier injection is a viable mechanism for optical switching in InGaAsP material, providing polarization and wavelength independent operation at response speeds on the order of nanoseconds.

Two mask layouts and several wafer layer structures were designed to study carrier injection and optimize performance of DOS devices. Using Mach-Zehnder and FabryPerot interferometer test structures, the relationship between refractive index modification and applied current density was characterized. It was determined that carrier lifetime and material bandgap play vital roles in determining switching contrast, switching speed, propagation loss, and driving power. The primary conclusion is that the switching speed is limited by Shockley-Reed-Hall carrier recombination times, and that increasing defect density reduces switching times at the expense of increasing required switching current. Alternatively, switching times may be reduced through over-driving. The dependence of refractive index modification on InGaAsP alloy was also studied, with the conclusion that a smaller bandgap material provides larger index changes at smaller current densities, with minimal detriment from absorption losses.

Several variations of the DOS device design were investigated, including devices with different waveguide width, branching angle, electrode shape, method of electrical isolation, and InGaAsP/InP heterostructure composition and doping. The optimized DOS devices demonstrated broadband operation, better than $25 \mathrm{~dB}$ contrast, less than $10 \mathrm{kA} / \mathrm{cm}^{2}$ switching current density, and an optical response time of less than $3 \mathrm{~ns}$. Additionally, faster response was achieved by electro-optical actuation. This DOS design is a promising device for optical router configurations. They have been successfully cascaded in a crosspoint configuration to construct a $2 \times 2$ switch. 
A bend loss attenuator actuated by carrier injection was also demonstrated, with significant improvements in performance over a straight waveguide attenuator. The device exhibits better than $15 \mathrm{~dB}$ modulation and $<20$ nanoseconds response time. The attenuator design is compact and can replace existing connection waveguides in a photonic integrated circuit, allowing it to be easily integrated. 


\section{Acknowledgements}

I would like to sincerely thank my supervisors, Dr. Siegfried Janz of the Institute for Microstructural Sciences, NRC, and Professor Barry Syrett of the Department of Electronics, Carleton University. They have provided constant support and constructive suggestions over the past years. I very much appreciate their concern for my wellbeing.

I would also like to recognize Dr. Pedro Barrios, for his expertise in fabricating all of the many devices in this thesis work and for his helpful advice. Thanks also go to my colleague, Shawkat Abdalla, for his support; Dr. Ilya Golub for his guidance; and Dr. André Delâge and Dr. Ross McKinnon for their simulation contributions as well as their valuable discussions and input. I am grateful to all of the researchers and students in IMS at NRC, and to the professors on my committee.

Finally, I would like to acknowledge my family for their encouragement and confidence in me. Thanks Eddie, Mom, Dad, and Baby. 


\section{Contents}

$\begin{array}{lll}\text { ABSTRACT } & \text { II }\end{array}$

ACKNOWLEDGEMENTS _ IV

CONTENTS $\quad$ V

$\begin{array}{ll}\text { LIST OF TABLES VII } & \text { VII }\end{array}$

$\begin{array}{lll}\text { LIST OF FIGURES VIII } & \text { VIG }\end{array}$

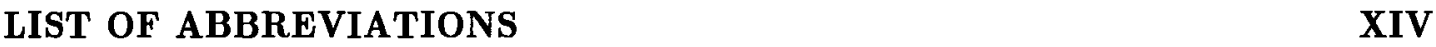

1 INTRODUCTION 1

1.1 Why Optical Switching ....................................................... 1

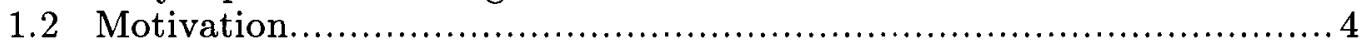

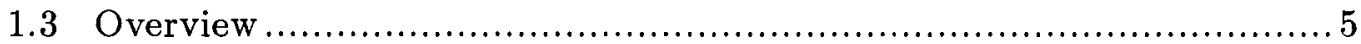

2 OPTICAL SWITCHING $\quad \mathbf{7}$

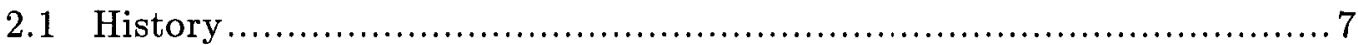

2.2 Review: Switching Mechanisms ....................................... 9

2.2.1 Carrier injection ...................................................... 9

2.2.2 Electro-optical ................................................... 10

2.2 .3 Thermo-optical ................................................... 11

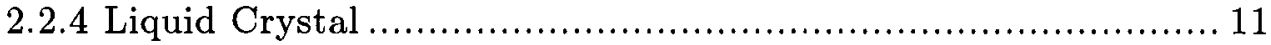

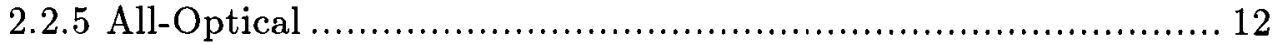

2.3 Literature Review: Switch Designs ................................... 13

2.3.1 Photonic crystal switches ........................................ 14

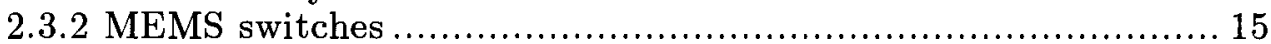

2.3.3 Integrated optical switches ........................................... 17

2.3.4 Waveguide Attenuators............................................ 23

2.4 Summary .................................................................. 24

3 RECONFIGURABLE WAVEGUIDE DIGITAL OPTICAL SWITCH 25

3.1 Device Design ........................................................... 25

3.1.1 Effective Index Method ............................................ 25

3.1.2 Device Configuration and Operation ................................ 26

3.1.3 Advantages of RW-DOS Design.................................. 28

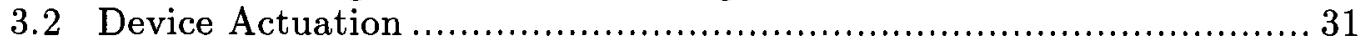

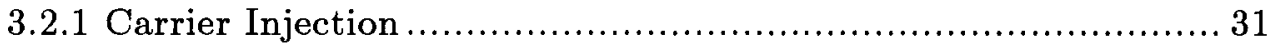

3.2.2 Quantum Confined Stark Effect................................ 35

3.2.3 Thermal Gradient.................................................... 36

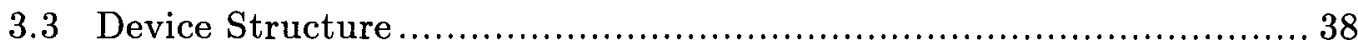

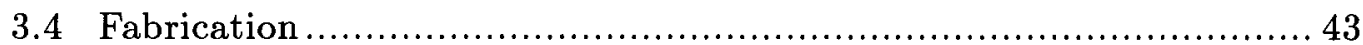

3.5 Device Modeling ....................................................... 45

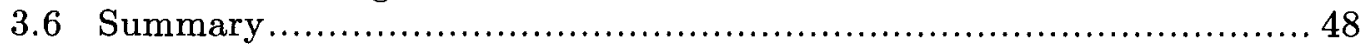


4 STUDY OF CARRIER INJECTION

4.1 Characterization Tools and Techniques................................ 50

4.1 .1 Experimental setup ......................................... 50

4.1.2 Mach-Zehnder interferometer index measurements............... 52

4.1.3 Fabry Perot interferometer index measurements.................. 55

4.2 Modeling ..................................................................... 56

4.2 .1 Carrier Lifetime................................................. 56

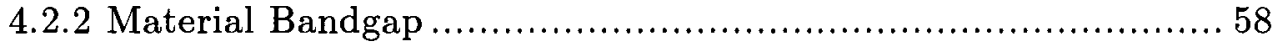

4.2.3 Carrier Diffusion.................................................. 59

4.3 Experimental Results ............................................... 61

4.4 Correction for Carrier Diffusion ........................................6 66

4.5 Quantum Well Structures ............................................... 68

4.5.1 C-band broadband measurements ............................. 70

4.5.2 L-band single wavelength measurements .......................... 70

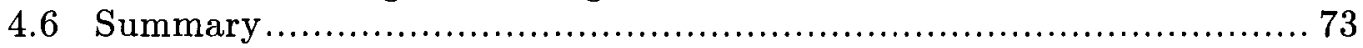

5 CHARACTERIZATION OF RW-DOS DEVICES

5.1 Device Design Parameters .................................................... 75

5.2 Electrical Isolation of Electrodes......................................... 77

5.2 .1 Electrode Isolation ........................................... 79

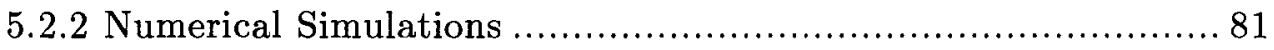

5.2 .3 Experimental Results ......................................... 85

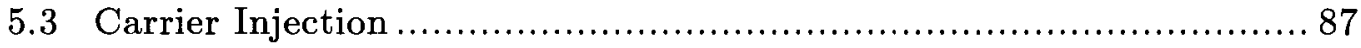

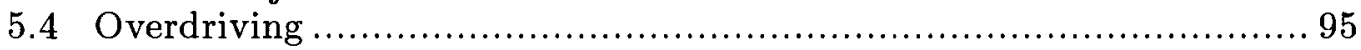

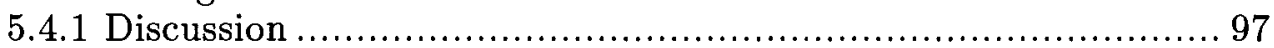

5.5 Quantum Well Implementation ........................................... 98

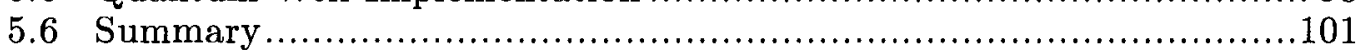

6 CHARACTERIZATION OF ADDITIONAL CARRIER INJECTION DEVICES $\quad \mathbf{1 0 3}$

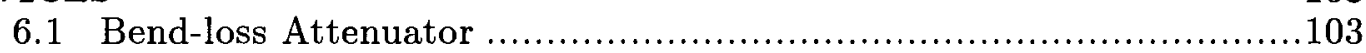

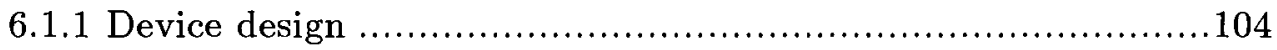

6.1.2 Simulations ........................................................ 106

6.1.3 Experimental results ..................................................108

6.2 Cascaded Switches ....................................................... 113

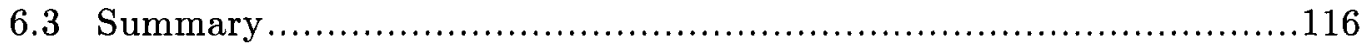

7 CONCLUSIONS AND FUTURE WORK $\quad 117$

7.1 Summary and Conclusions ...........................................117

7.1.1 Study of Carrier Injection ........................................... 117

7.1.2 Device Results ........................................................118

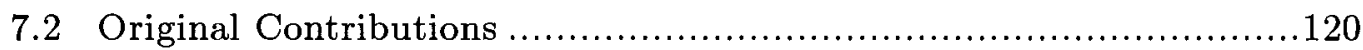

7.3 Directions of Future Research ...................................... 122

7.3.1 Improvements to InGaAsP/InP RW-DOS Devices ................123

7.3 .2 Optical Switches in SOI ........................................124

$\begin{array}{ll}\text { APPENDIX } & 126\end{array}$

$\begin{array}{lr}\text { REFERENCES } & 128\end{array}$

vi 


\section{List of Tables}

Table 3-1 Designed waveguide contrast for TE and TM polarization for the three InGaAsP alloys 41

Table 4-1 Material index change versus current density values from MZ interferometer fringe data with TE polarized broadband light 65

Table 4-2 Index modulation of modal index per applied current density $\mathrm{d} n / \mathrm{d} j$ in $\mathrm{kA}^{-1} \mathrm{~cm}^{2}$ for MOCVD and MBE grown wafers............................... 70 


\section{List of Figures}

Figure 1-1 "The evolution toward photonic networking" [1] .....................

Figure 2-1 Illustration of liquid crystal phase

[http://plc.cwru.edu/tutorial/enhanced/files/textbook.htm]

Figure 2-2 Illustration of dispersion relation (band structure), frequency $\omega$ versus wave number $\mathrm{k}$, of photonic crystal with two bandgaps shaded in grey. ... 15

Figure 2-3 Illustration of 3D MEMS OXC with optical signals focused by a lens array onto the mirror array which is positioned to switch the signals to the desired output fibers [20].

Figure 2-4 Layout of SOA switch with SOA gates on each output branch...... 17

Figure 2-5 Layout of MMI switch for which an signal applied to the dashed rectangular electrode will modify the refractive index so that input light interferes to couple to the desired output port. The electrode shape is only an example.

Figure 2-6 Layout of DC switch for which an signal applied to the dashed rectangle will modify the refractive index so that input light couples to the desired output port.

Figure 2-7 Layout of a MZI switch with an actuation element on the bottom arm to control phase so that the interference between the two arms cause the signal to couple to the desired output port.

Figure 2-8 Illustration of ring resonator switch with input at bottom left and actuation signal applied to dashed circle to control resonance so that the signal is switched to either output.

Figure 2-9 Illustration of $\mathrm{Y}$-splitter switch with input at left and actuation signal applied to dashed rectangle to control refractive index so that the signal is switched to either output.

Figure 2-10 Illustration of X-splitter switch with input at left (a) switching to top output arm with no applied bias and (b) switching to bottom output arm as the input signal reflects off the edge of dashed rectangular control pad....22

Figure 3-1 Illustration of ridge, slab, and mode effective refractive indices..... 26

Figure 3-2 (a) Configuration of the proposed DOS Y-junction switch with input at the left and outputs at the right and (b) Effective waveguide configuration when the index under the biased electrode matches the refractive index of the surrounding slab region. 


\section{LIST OF FIGURES}

Figure 3-3 (a) Adiabatic mode evolution Y-branch switch design with tapered waveguides and (b) a TIR X-branch switch with bowtie shape electrodes, designed to allow larger branching angles [45]....

Figure 3-4 Switching contrast in $\mathrm{dB}$ between arms of RW-DOS, modal evolution, and TIR switches versus product of index modulation and electrode length.

Figure 3-5 Model of refractive index change as a function of carrier concentration for $\mathrm{Q}=1.3 \mu \mathrm{m}$ InGaAsP alloy according to [58-60]. The contributions of free carrier absorption, band-gap shrinkage (bgs), and bandfilling after bandgap shrinkage (bf $+\mathrm{bgs})$, are shown in dashed, dotted, and solid lines respectively.

Figure 3-6 Illustration of tilting of the bands with applied field [62] (a) resulting in a redshift [63] (b) of the exciton transition peak in the absorption spectrum.

Figure 3-7 Calculated temperature variation with horizontal (in the wafer plane indicated by dashed line) distance in an InGaAsP/InP waveguide with a 5 $\mu \mathrm{m}$ wide heat source (centered at $\mathrm{x}=0 \mu \mathrm{m}$ ) embedded in the InGaAsP core layer, at different times after the heat source is turned on. The dashed line indicates the edge of the heat generating region [67]. 37

Figure 3-8 Measured output intensities vs. time (a) and peak current (b) for the biased and unbiased arms of the DOS switch for a $1.5 \mu$ s applied current pulse [67].... 38

Figure 3-9 Schematic diagram illustrating the layer structure of the CBE grown InGaAsP/InP wafers. 39

Figure 3-10 Schematic diagram illustrating the layer structure of the MOCVD grown InGaAsP/InP wafers.

Figure 3-11 Schematic diagram illustrating the layer structure of the MBE grown InGaAsP/InP wafers.

Figure 3-12 Energy band structure of the three QW superlattice structures (a) RQWa (b) RQWb, and (c) 3SQW.

Figure 3-13 (bottom) Configuration of DOS with (a) physical isolation trench and (b) ion implant isolation and (top) cross-section of the DOS at the position indicated by the dashed line. 44

Figure 3-14 BPM simulation results showing normalized optical output power of $\mathrm{TE}$ polarization as a function of applied negative index change in the biased (bottom) and unbiased (top) arms, comparing layer designs for (a) MOCVD and (b) MBE wafers with InGaAsP cores of bandgaps $\mathrm{Q}=1.2 \mu \mathrm{m}, 1.3 \mu \mathrm{m}$, and $1.4 \mu \mathrm{m}$. 


\section{LIST OF FIGURES}

Figure 3-15. BPM simulation results showing calculated switch contrast ratio of power between the two DOS arms as a function of applied negative index change comparing $\mathrm{TE}$ and $\mathrm{TM}$ polarizations for $\mathrm{Q}=1.3 \mu \mathrm{m}$.

Figure 3-16 Band diagram through the center of the waveguide ridge from the top p-doped layer down, at (s) zero applied bias and (b) with $1.4 \mathrm{~V}$ forward bias.

Figure 4-1 Experimental setup for device characterization illustrating the optical source and detection systems, and the electrical signal system............. 52

Figure 4-2 Plot of BPM generated fringes of $M Z$ interferometer versus ridge

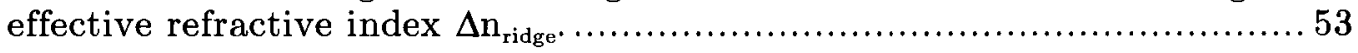

Figure 4-3 Plot of Matlab simulation results comparing MZ fringes using a broadband (solid line) and a single wavelength (dotted line) source........ 54

Figure 4-4 Carrier lifetimes as a function of carrier concentration modeled with Auger, Shockley-Reed-Hall (SRH), and optical recombination. The total lifetime is shown by the thick solid black line, whereas the lifetime with no SRH mechanism (i.e. only Auger and optical recombination) is shown by the thin solid black line.

Figure 4-5 Modeled values of index change at $1550 \mathrm{~nm}$ versus carrier concentration $N$ for InGaAsP of bandgap (Q) $1.4 \mu \mathrm{m}, 1.3 \mu \mathrm{m}$, and $1.2 \mu \mathrm{m}$ using models of bandgap shrinkage according to (a) Weber [58] and (b) Botteldooren and Baets [60].

Figure 4-6 Modeled hole concentration along the cross section through a $\mathrm{Q}=1.3 \mu \mathrm{m}$ InGaAsP core layer as a function of lateral position.

Figure 4-7 Illustration of Mach-Zehnder interferometer device.

Figure 4-8 Measured optical output power from a MZ waveguide interferometer driven by a triangular current pulse, for a wafer grown by MOCVD with a core layer alloy of bandgap Q1.4 $\mu \mathrm{m}$.

Figure 4-9 Variation of index change per current density with wavelength from theory (lines) and from measurements of MZ interferometer fringes for devices on MOCVD grown $\mathrm{Q}=1.4 \mu \mathrm{m}, 1.3 \mu \mathrm{m}$, and $1.2 \mu \mathrm{m}$ wafers (points). 63

Figure 4-10 Index change versus current density calculated from Fabry-Perot (FP) fringes measured using TE polarized broadband light for devices on $\mathrm{Q}=1.4 \mu \mathrm{m}$ wafers with waveguides widths $5 \mu \mathrm{m}, 4 \mu \mathrm{m}$, and $3 \mu \mathrm{m}$.

Figure 4-11 Modeled (lines) and measured (points) values of index change at $1550 \mathrm{~nm}$ versus carrier concentrations for MOCVD-grown InGaAsP of bandgap (Q) $1.4 \mu \mathrm{m}, 1.3 \mu \mathrm{m}$, and $1.2 \mu \mathrm{m}$ 


\section{LIST OF FIGURES}

Figure 4-12 Spectral transmission of (a) the three QW wafers corresponding to the designs in Figure 3-12, and (b) RQWa with applied reverse bias of $0 \mathrm{~V}$, $1 \mathrm{~V}$, and $-2 \mathrm{~V}$.

Figure 4-13 Plots of index change versus current density calculated from observed $\mathrm{FP}$ and MZ interference fringes for the QW wafers (a) RQWa (b) RQWb and (c) $3 \mathrm{SQW}$. The source was a tunable laser at $1600 \mathrm{~nm}$.

Figure 4-14 Plots of index change versus current density calculated from observed MZ interference fringes for QW wafer RQWa in comparison with bulk wafers in TE polarization.

Figure 5-1 (a) Calculated output power in the biased (bottom) and unbiased (top) RW-DOS arms as a function of the induced refractive index change for three branching angles, and (b) the corresponding output power measured in fabricated RW-DOS devices as a function of bias current. ...

Figure 5-2 Comparison of fabrication steps of electrode isolation by (a) ionimplant and (b) trench isolation. .......................................... 78

Figure 5-3 Calculated ion implantation concentrations versus depth. 80

Figure 5-4 SEM images of a DOS isolation trench (a) from the top, (b) magnification of the area in the dashed box, and (b) in cross section.

Figure 5-5 Calculated distribution of holes in the core layer for implant and trench isolation at cross sections where the electrodes are (a) $3 \mu \mathrm{m}$, (b) 1.5 $\mu \mathrm{m}$, and (b) $0.5 \mu \mathrm{m}$ wide. The dotted vertical lines show the position of the overlying electrode. 82

Figure 5-6 Drift diffusion modeling of incomplete etching of trench. 83

Figure 5-7 Illustration of current spreading and carrier diffusion in structures with (a) implant isolation and (b) trench isolation.

Figure 5-8 BPM results comparing $0.9^{\circ}$ branching angle DOS without (a) and with (b) $0.5 \mu \mathrm{m}$ wide isolation trench at junction. ......................... 85

Figure 5-9 Experimental measurements of current versus voltage between the contacts.

Figure 5-10 Experimental measurements of optical power in biased and unbiased arms of DOS devices with branching angle of $1.2^{\circ}$.

Figure 5-11 Experimental data from RW-DOS devices on MOCVD wafers with core of alloy (a) Q1.4 $\mu \mathrm{m}$, (b) Q1.3 $\mu \mathrm{m}$, and (c) Q1.2 $\mu \mathrm{m}$. TE polarization is shown in the left column and, and TM in the right column. The bias current pulse is $100 \mathrm{~ns}$ long with an amplitude of $12 \mathrm{~mA}$. 88 


\section{LIST OF FIGURES}

Figure 5-12 Experimental data from digital optical switch (DOS) devices on (a) MOCVD and (b) MBE wafers switched with $200 \mathrm{~ns}$ pulses of $5 \mathrm{~mA} \ldots \ldots . .90$

Figure 5-13 Experimental data from digital optical switch (DOS) devices on MBE and MOCVD wafers.

Figure 5-14 Experimental data comparing the (a) optical output in TE polarization and (b) contrast of $1.35^{\circ} \mathrm{DOS}$ devices with core alloys of $\mathrm{Q}=1.2$ $\mu \mathrm{m}, 1.3 \mu \mathrm{m}$, and $1.4 \mu \mathrm{m}$.

Figure 5-15 Experimental data in TE polarization of index change versus current density as calculated from MZ fringes. .94

Figure 5-16 Experimental output from switched DOS comparing no overdrive (12 $\mathrm{mA})$ and overdriving $(22 \mathrm{~mA}$ and reverse bias of $-1.5 \mathrm{~V})$ with a $50 \mathrm{~ns}$ pulse for (a) TE and (b) TM polarizations. The core alloy is InGaAsP with $\mathrm{Q}=1.4$

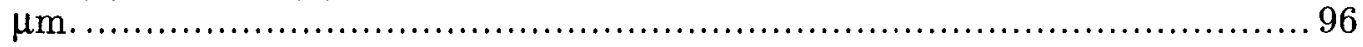

Figure 5-17 Oscilloscope trace of $0.78^{\circ}$ DOS on wafer RQWa in TE polarization. 98

Figure 5-18 Experimental results from the RQWa wafer showing (a) optical output power versus time and (b) switching contrast versus applied reverse bias. .... 100

Figure 6-1 BPM simulation results of a bend loss attenuator with input at the left and output at the right showing (a) propagation through the unmodulated device, and (b) propagation with current applied to the electrode causing a negative refractive index change in the waveguide below.

Figure 6-2 BPM results showing loss due to the bend structure for bends of different radii.

Figure 6-3 BPM results showing loss due to mode mismatch for straight and bent waveguide attenuators.

Figure 6-4 BPM results for straight and bent waveguide attenuators. The calculation is for TE polarization and includes carrier induced absorption losses.

Figure 6-5 Experimental results for straight and bent waveguide attenuators. The inset shows the bent waveguide data plotted over a larger range. ... 108

Figure 6-6 (a) BPM results for bend loss attenuators and (b) experimental results of the same attenuators for $\mathrm{TE}$ polarization.

Figure 6-7 (a) Drift diffusion modeling of carrier concentration across the waveguide, and (b) the corresponding temperature increase due to the thermo-optical effect. 


\section{LIST OF FIGURES}

Figure 6-8 Modeling results showing effective refractive index profile due to carrier injection (solid line) and both carrier and thermal (dashed line) effects at applied currents of $10 \mathrm{~mA}, 50 \mathrm{~mA}$, and $100 \mathrm{~mA}$.

Figure 6-9 Experimental measurements of output power measured from fabricated bend loss attenuator and voltage from pulse generator versus time for an applied pulse length of $100 \mathrm{~ns}$.

Figure 6-10 Illustration of $2 \times 2$ switch in (a) cross configuration and (b) bar configuration. The solid electrodes indicate (forward) biased electrodes. 113

Figure 6-11 Plots of relative output power from ports of a $2 \times 2$ switch when signal is input to the (a) top arm and (b) bottom arm. Bar and cross configurations are indicated....

Figure 7-1 Illustration of buried waveguide-heterostructure. 


\section{List of Abbreviations}

$\begin{aligned} \text { 2D } & \text { two dimensional } \\ \text { 3D } & \text { three dimensional } \\ \text { ASE } & \text { amplified spontaneous emission } \\ \text { ATM } & \text { asynchronous transfer mode } \\ \text { BGS } & \text { bandgap shrinkage } \\ \text { BPM } & \text { beam propagation method } \\ \text { CAIBE } & \text { chemically assisted ion beam etch } \\ \text { CBE } & \text { chemical beam epitaxy } \\ \text { DC } & \text { directional coupler } \\ \text { DOS } & \text { digital optical switch } \\ \text { DUT } & \text { device under test } \\ \text { DWDM } & \text { dense wavelength division multiplexing } \\ \text { EDFA } & \text { erbium doped fiber amplifier } \\ \text { EM } & \text { electromagnetic } \\ \text { EO } & \text { electro-optic } \\ \text { FP } & \text { Fabry-Perot } \\ \text { FTTP } & \text { fiber-to-the-premises } \\ \text { ICP-RIE } & \text { inductively coupled plasma reactive ion etching } \\ \text { InP } & \text { indium phosphide } \\ \text { IP } & \text { internet protocol } \\ \text { IR } & \text { infrared } \\ \text { LC } & \text { liquid crystal } \\ \text { MBE } & \text { molecular beam epitaxy } \\ \text { MEMS } & \text { micro-electromechanical system } \\ \text { MMI } & \text { multimode interferometer } \\ \text { MOCVD } & \text { metal-organic chemical vapour deposition } \\ \text { MPLS } & \text { multiprotocol label switching } \\ \text { MZ } & \text { Mach-Zehnder } \\ \text { MZI } & \text { Mach-Zehnder interferometer } \\ \text { NA } & \text { numerical aperture } \\ \text { O } & \text { oxygen ion } \\ \text { OXC } & \text { optical cross connect } \\ \text { PDL } & \text { polarization dependent loss } \\ \text { PLC } & \text { planar lightwave circuit } \\ \text { QCSE } & \text { quantum confined Stark effect } \\ \text { QoS } & \text { quality of service } \\ \text { ROADM } & \text { reconfigurable optical add-drop multiplexer } \\ \text { RW-DOS } & \text { reconfigurable waveguide digital optical switch } \\ \text { SEM } & \text { scanning electron microscope } \\ \text { SOA } & \text { semiconductor optical amplifier } \\ \text { SOI } & \text { silicon-on-insulator } \\ \text { SRH } & \text { Shockley-Reed-Hall } \\ \text { SONET } & \text { synchronous optical networking } \\ \text { TIR } & \text { total internal reflection } \\ \text { VOA } & \text { variable optical attenuator } \\ & \end{aligned}$

xiv 


\section{Chapter 1}

\section{INTRODUCTION}

\subsection{Why Optical Switching}

All of the arguments in favour of optical switches over their electrical counterparts are as valid today as they were during the height of the optical technology boom. Optical switches eliminate optical-to-electrical conversion, thereby reducing the delays currently suffered by information passing through network switching nodes. In addition, an optical implementation offers reduced footprint, weight, and power requirements compared to an electrical implementation. Yet despite these advantages, the holy grail of internet protocol (IP) over an all-optical core has yet to manifest itself. So is there any reason to believe that it ever will?

The replacement of electronic technology by optical was prompted by the need for increased bandwidth. While bandwidth requirements do still continue to grow, a stronger demand has overtaken bandwidth: the demand for service. A simple pointto-point connection is no longer sufficient, no matter its speed or capacity. Users are requiring quality-of-service (QoS) and multiple service capabilities. In particular, the introduction of triple play service (high speed internet, video on demand, and telephony) has placed varying constraints on network performance and has led to the convergence of network layers. New network protocols have emerged, such as multiprotocol label switching (MPLS) (Figure 1-1(b)), which employs a control system to 
set up specific routes or Label Switched Paths (LSPs) based on network contraints. Each label-switching router (LSR) attaches a simple forwarding label to IP packets to direct its next hop, streamlining the routing process for all-optical cores while maintaining the traffic engineering capabilities of older asynchronous transfer mode (ATM) and synchronous optical networking (SONET) protocols.

This convergence of network layers has led to a simplification but at the same time improvement of network intelligence and control. A key factor behind this has been device integration, of which optical switching devices is a prime example. As of this date, MPLS has already been adopted by the marketplace to replace ATM. In future, as MPLS and generalized MPLS (GMPLS) become prevalent, it is predicted that the switching functions of SONET will be replaced by optical cross connects (Figure 1-1(c) and $(d))$.

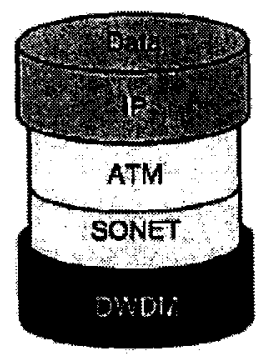

(a)

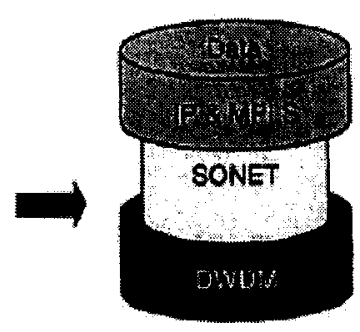

(b)
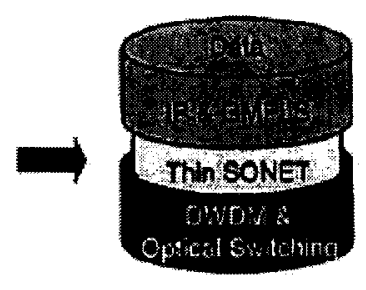

(c)

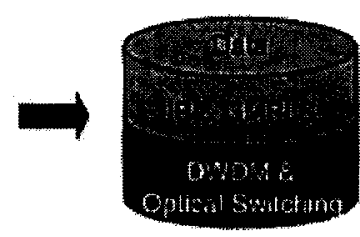

(d)

Figure 1-1 "The evolution toward photonic networking" [1].

Thus, within the past few years, the optical telecommunications industry has moved away from the development of large port-count optical switches that provide merely switching functionality. The movement has been towards integration of smaller portcount switches with other dense wavelength division multiplexing (DWDM) components. One such example is Siemens' shift in focus from their two-dimensional (2D) microelectromechanical systems (MEMS)-based TransXpress product to their Multiservice Integrator (MSI-160). The name itself expresses the intended versatility of the product, which integrates multiplexing and crossconnect functionality.

Currently, optical switches have found niche markets in several areas. Test equipment and instrumentation in laboratories is an example of one of the larger markets. 
Another example is as remote test, monitoring, and even reconfiguration equipment within optical networks. Optical switches are used, for example, to reconfigure networks to compensate for fiber breaks or to switch to diagnostic equipment. The use of switching for this purpose has allowed automatic and remote network diagnosis and restoration. In particular, testing and fiber management of the countless access networks that will provide fiber-to-the-premises (FTTP) will benefit greatly from remotely controlled optical switches. Optical switching for routing has still not settled between a large-scale optical cross connect (OXC) implementation, and a solution based on smaller grooming switches built into reconfigurable add-drop multiplexers (ROADM). Both are commercially available. Finally, Japanese companies such as Nozomi Photonics and Yokogawa Electric are racing to provide optical switches for burst or packet switching of Ethernet and IP traffic. In all, the optical switching market is one of the fastest growing ones within the optical equipment industry, with 25 percent growth in just one quarter at the end of last year [2].

In terms of convergence, the collapse of networking layers is limited by the ability to perform optical packet switching, which is currently limited as much if not more by optical storage capabilities as by optical switching speed. However, routing at the IP level using MPLS is ripe for optical implementation as it requires only nanosecond switching, which is feasible with current optical switching technology, and has reduced buffering requirements. In fact, leading telecommunications companies Cisco, Lucent, and JDS Uniphase, among others, are now working on Defense Advanced Research Projects Agency (DARPA) funded programs ${ }^{1}$ to develop an all-optical terabit router. A crucial factor for these projects will be the extremely high degree of optical device integration required.

Current injection based integrated optical switches are well positioned to fill this new role. They have well established reliability, the active element being a simple diode, as opposed to MEMS-based devices which are mechanical systems with moving parts. Additionally, the III-V material system is compatible with existing semiconductor

\footnotetext{
${ }^{1}$ Two DARPA optical router programs are the Label Switched Optical Router (LASOR) and Integrated Router Interconnected Spectrally (IRIS)
} 
manufacturing. Finally, switching speeds are in the target nanosecond range for MPLS.

\subsection{Motivation}

The present DOS project is a collaboration between Prof. B. Syrett, his graduate students from Carleton University, and several National Research Council (NRC) scientists in the Nanofabrication and Optoelectronic Devices groups of the Institute for Microstructural Sciences (IMS). The title of the originally funded project was "High Speed Reconfigurable Optical Router and its Applications to Optical Switching in IP Networks", and it was funded by the National Capital Institute of Telecommunications (NCIT). As such, one goal of the project was to integrate the DOS into a multi-port photonic switching circuit.

This work in this thesis was motivated by the demand for an integrable optical switch with an actuation speed on the order of a nanosecond, which would be suitable for use in an optical router operating at the IP or MPLS level. The indium gallium arsenide phosphide (InGaAsP) on indium phosphide (InP) material system was chosen for its source and detector functionalities, and carrier injection and electro-optical modulation were identified as actuation mechanisms satisfying nanoseconds speeds.

The primary device design is a $1 \times 2$ digital optical switch (DOS) based on the reconfiguration of the waveguides at the junction of a $1 \times 2$ Y-branch, a concept developed and patented [3] by J.J. He and A. Delâge at the Institute for Microstructural Sciences (IMS) at the National Research Council (NRC) of Canada. The novel shape of the electrodes allows switching to be accomplished by a small, local modulation of the waveguide refractive index. This index modulation may be accomplished through a variety of methods, including electrical carrier injection, thermo-optical, electro-optical, or nonlinear optical effects.

The particular aims of this thesis were the in-depth study of carrier-injection index modifications and the demonstration of the DOS device employing carrier injection in 
InP. However, the development of optical switching and routing technology must necessarily include integration of optical components and functionalities. So the secondary aim of this thesis is the exploration of accompanying devices with which switches may be integrated, as well as the study of integration of discrete switch devices into larger cascaded architectures. Additionally, electro-optical actuation was investigated for sub-nanosecond operation.

To these ends we have explored:

1. the carrier injection mechanism in the $\operatorname{InGaAsP} / \mathrm{InP}$ material system, based on systematic experimental measurements,

2. digital optical switch (DOS) and variable optical attenuator (VOA) designs using carrier injection and electro-optic actuation mechanisms, and

3. integration of multiple DOS switches on a single chip - the first step to forming integrated photonic circuits.

\subsection{Overview}

This thesis aims to contribute in two areas: one is the development of waveguide based integrated photonic devices on InGaAsP/InP, in particular a novel digital optical switch design, and the other is the study of carrier injection dynamics in InP to optimize carrier injection actuated devices.

Chapter 2 introduces the past and current research into optical switch and waveguide attenuator design, and describes the different mechanisms employed in their actuation. In Chapter 3, the DOS device structure and operating principles are described, as well as the details as regards design and fabrication. Chapter 4 presents the modeling and study of carrier injection in bulk and quantum well InGaAsP, including a description of experimental methods and equipment. The results of performance optimization of our carrier injection actuation reconfigurable waveguide digital optical switch devices 
are presented in Chapter 5. As well, the results of electro-optically modulated quantum well switches are presented. Chapter 6 details the characterization and results of additional carrier injection devices, in particular bend-loss attenuators, which are an essential component in integrated optical circuits, and $2 \times 2$ cascaded switches, which are the first step towards large scale integration. Conclusions in Chapter 7 point future work towards fine-tuning switch design to meet commercial criteria and exploring designs for the semiconductor on insulator material system. Also included here is a summary of original contributions. 


\section{Chapter 2}

\section{OPTICAL SWITCHING}

\subsection{History}

The replacement of electronics by photonics in telecommunication and data transmission links and networks was forced by the demand for increasing information carrying capacity. However, one fundamental network component, the simple space switch that directs a signal to a desired destination, has yet to be entirely replaced by a photonic counterpart. Such space switches are useful in many network applications, including protection switching, network reconfiguration, and dynamic lightpath setup. Each of these has different requirements and so different switching technologies have emerged in each application as the best candidate.

Optical switches were first applied to provide alternative routing in the event of network failure. Such protection switches called for small port-count (1x2 or $2 \times 2)$ and slow switching speeds (tens of milliseconds), thus thermo-optic waveguide-based switches and even optomechanical fiber switches were sufficient. Subsequently, the desire to remotely reconfigure networks led to the installation of optical cross connects (OXC) for mesh topologies, and reconfigurable add-drop multiplexers (ROADMs) for ring topologies.

Provisioning of lightpaths in mesh networks requires large port-counts and currently OXCs of up to $160 \times 160$ ports are commercially available. In this area, three dimen- 
sional (3D) MEMS based switches have significant advantages. Several OXCs based on MEMS are available from companies such as Glimmerglass Networks. Mirrors in an array are tilted to redirect optical signals to desired output ports. The disadvantages of MEMS solutions are slow speed, low yield, large footprint, and packaging complexity. There is also often a perception that mechanical systems are unreliable, and MEMS do have complications with stiction, shock, vibration, and manufacturing tolerance. Still, they are the leading technology in the OXC market and have not only nearly replaced optomechanical implementations, but they are also becoming prevalent in the protection switching market. In comparison, OXCs implemented from integrated optical switches are far fewer in number. These guided wave devices are desirable for their integrability and undesirable for their high loss and lack of scalability. ROADMs on the other hand, which add and drop specific traffic channels from a network, require simple $1 \times 2$ or $2 \times 2$ switching, thus opening the door for integrated optical switches.

Integrated optical switches cover a wide variety of devices, including all planar lightwave circuit (PLC) devices. For example, thermo-optic PLC switches are available on silica-on-silicon as well as polymer, and they switch at speeds of several milliseconds (ms). Research groups are also investigating switching in photonic bandgap crystals. This thesis itself proposes an electro-optically driven switch in indium phosphide (InP). In comparison to MEMS, integrated optic switches are a less mature technology, but a 2002 Communications Industry Researchers (CIR) market report predicts that integrated optics-based switches will soon account for " 12 percent of the optical-switching-device market" [4]. One driving factor behind this trend is the speed advantage that integrated optical switches offer.

IP over an all-optical core is slowly becoming a reality, and the next step towards its realization is the optical switching of data. There is already a platform on hand which facilitates the implementation of IP in the optical domain: MPLS provides a control plane to configure optical devices that cannot directly read routing information. Further driving the development of integrated optical switches is the imminence of optical burst and packet switching, which will allow sub-wavelength switching of clusters (burst) or individual packets respectively. These switching systems require 
speeds of several nanoseconds or less. Such high speed is currently feasible only by solid-state switching. Indeed, Japanese companies are aggressively developing burst switching technology with the anticipation of deployment within the next two years [5]. As well, packet switching is being developed for use with optical interconnects in high speed computing [6].

Finally, beyond OXCs, ROADMs, and packet switching, another great challenge to agile all-optical networks will be the self-direction of optical signals. Such optical routing will be accomplished by a system comprising a switching fabric along with the controls to route signals through that fabric based on reading information encoded in the optical signals. Thus integration will be even more essential for future optical switching technologies.

\subsection{Review: Switching Mechanisms}

As mentioned previously, there are many different methods of actuating waveguidebased switches. The choice of method is largely dependent upon the material system, and it will influence device design, switching speed, and other aspects of device performance. In this work, I focus on actuation by carrier injection, although electrooptical and thermo-optical methods are also examined.

\subsubsection{Carrier injection}

An electric field injects free carriers into semiconductor materials, inducing an index modification through the effects of free carrier absorption, bandfilling, and bandgap shrinkage. These effects are explained in more detail in Section 3.2.1. Carrier induced index changes are wavelength and polarization insensitive. They have an actuation speed determined by carrier lifetimes, generally on the order of nanoseconds; however this can be reduced through for example the application of electric fields to sweep out carriers. 


\subsubsection{Electro-optical}

Electro-optical (EO) effects are those in which a change in refractive index is generated by an applied electric field. The EO response speed is dependent upon the EO material but is in general a very fast response, and so switching speed is limited by the driving circuit, usually to tens of picoseconds. The index change may be considered as either a direct electric field induced polarization of the material, or a change in material absorption which is related to an index change through Kramers-Krönig transformation [7]. Direct effects include the linear Pockels effect and the second order-Kerr effect. Absorption changes include the Franz-Keldysh and Stark effects in bulk, and the quantum-confined Stark effect (QCSE) in quantum well materials.

Index change can be described as a series expansion dependent upon the electric field, due to the relation between polarization density $P$ electric field $E$ through electric susceptibility $\chi$ :

$$
P=\varepsilon_{0} \chi^{(1)} E+\varepsilon_{0} \chi^{(2)} E E+\varepsilon_{0} \chi^{(3)} E E E+\ldots
$$

where $\varepsilon_{0}$ is the vacuum permittivity $=8.854 \times 10^{-12} \mathrm{~F} / \mathrm{m}$. Refractive index $n$ is related to susceptibility through:

$$
n=\sqrt{\varepsilon_{r} \mu_{r}}=\sqrt{(1+\chi) \mu_{r}}
$$

where $\varepsilon_{r}$ is the relative permittivity, and $\mu_{r}$, the relative permeability, is close to unity for nonmagnetic materials.

The linear terms relating index change to electric field describe the Pockels effect and are nonzero only for materials without inversion symmetry, such as lithium niobate. All materials exhibit the nonlinear Kerr effect but this effect is small and so high intensities, such as produced by short pulsed lasers, are required to induce any substantial change in refractive index. The Franz-Keldysh and Stark effects are also small. The former involves a change in absorption due to a change in electron/hole wave functions. The latter is analogous to the magnetic field induced Zeeman effect, in that an electric field breaks the degeneracy of electron energy states. This effect is 
greatly enhanced for electrons and holes bound together (excitons) in quantum well layers. Thus the quantum confined Stark effect is a common method of optical device actuation and is described in more detail in Section 3.2.2.

Transmission through semiconductor based EO materials generally incurs larger losses because design of the materials for large index modifications requires operation near the band edge, which results in higher absorption through the Urbach tail. Hybrid integration with lower loss materials provides higher transmission through passive waveguide sections and limits higher losses to the length of the electrodes. However this causes additional fabrication complexity and requires index matching to reduce losses at the material interfaces. In this manner, a Y-branch switch with extinction ratio better than $20 \mathrm{~dB}$ has been fabricated using low-loss polymer passive waveguides and EO polymer at the switching junction [8].

\subsubsection{Thermo-optical}

Heating causes refractive index modifications through a combination of thermal expansion of the lattice and changes to the electronic energy states. The former tends to cause a negative index change with increasing temperature, while the latter, which is dominant in semiconductor materials, has the opposite effect [9]. Thermo-optical effects operate on the order of milliseconds; however a thermal gradient heating effect inadvertently discovered and employed by researchers at IMS for switching reduces actuation times to hundreds of nanoseconds. This effect is described in more detail in Section 3.2.3.

\subsubsection{Liquid Crystal}

Liquid crystals (LC) are long polarizable molecules in a phase state between solid and liquid, wherein the molecules align along a director axis. This anisotropy makes liquid crystals birefringent. The molecules guide electric fields so that twisted crystals can rotate the polarization of incident light. An electric charge applied to liquid crystal molecules causes them to untwist and transmit incident light with the polarization unchanged. Thus an electrically-actuated free space switch can be constructed using liquid crystals and bulk optic polarization beam splitters [10]. Additionally, the 
rotation of the crystals alters its refractive index allowing it to be useful in interferometric waveguide based switches.

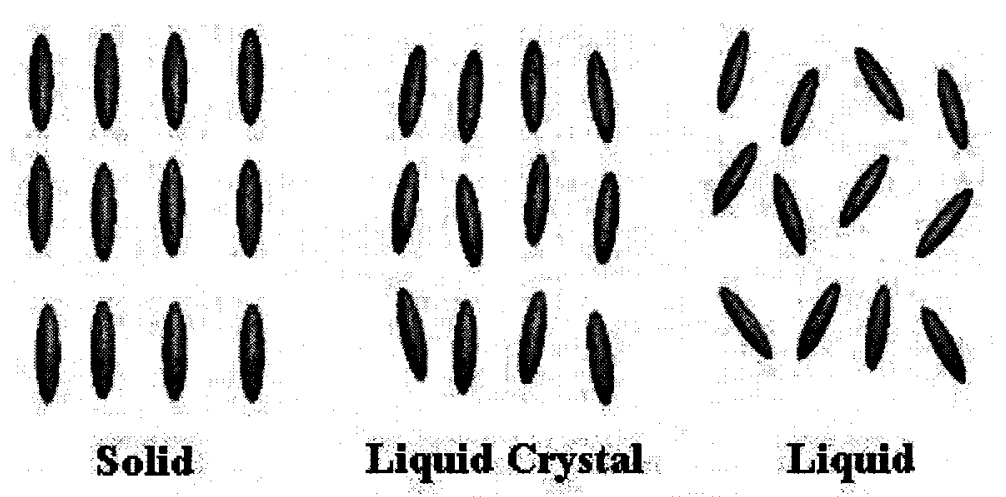

Figure 2-1 Illustration of liquid crystal phase

[http://plc.cwru.edu/tutorial/enhanced/files/textbook.htm].

LC response times range from microseconds to milliseconds, dependent upon such factors as LC material, thickness, and applied voltage. Furthermore, the large birefringence of LC materials introduces considerable polarization dependence.

\subsubsection{All-Optical}

Recently, there have been some developments in the area of all-optical switches. For such switches, the actuation signal is delivered optically, typically by a light beam absorbed through the top surface of the PLC. This mode of delivery negates the advantages of a PLC implementation and requires that the desired region of index modification be defined by either beam shaping, which requires additional optics, or an exposure window, which renders much of the optical power useless. Also, focusing and alignment of the control light are key to delivery of sufficient photon intensity to induce switching through electron-hole pair generation or nonlinear optical effects. Thus switching power depends largely on the efficiency of the optical power delivery and device design. Device switching speeds are generally limited by carrier lifetime in the case of carrier injection, and system response speeds in the case of nonlinear effects which have very fast response times.

One example of optical-actuation achieves switching by TIR at the junction of a Ybranch [11]. The reflecting interface is generated by photon-induced carrier generation. 
Another example is a directional-coupler-like design, in which a central waveguide between the arms of the directional coupler is modified by control light to decouple from the input waveguide [12]. Advantageously, in this design the optical control signal is delivered through an in-plane waveguide. Also well-suited to all-optical actuation is the ring resonator design, which requires a small index modification and a circular exposure area.

\subsection{Literature Review: Switch Designs}

The pace of research in optical switching has slowed considerably since the downturn of the telecommunications industry several years ago. Companies that had scrambled to enter the telecommunications switching market from such diverse fields as inkjet printing ${ }^{2}$ and graphic arts $^{3}$ have now returned to their own domains. However that is not to say that the field is stagnant. There are many optical switching implementations in early development stages and others that are suitable for fields other than telecommunications. For example, microfluidic switches flow various liquids through micro-channels to induce total internal reflection at switching points. Switching speeds are slow $(\sim 20 \mathrm{~ms})$ but the design is compatible with and suitable for biophotonic labs-on-a-chip [13]. Additionally, reflection/transmission switches are impractical for industry; however they are desirable for laboratory use because of their simple construction. The optical signal is delivered in free space to the switching material, which may be controlled to either reflect or transmit. The switching material may be for example a magnetic fluid whose refractive index is modified by an external magnetic field [14], or it may be a nonlinear optical (NLO) material controlled by a pump beam [15].

However, for telecommunications optical switching, response speed, port count, and device footprint have limited the competing technologies to only a few. MEMS-based

\footnotetext{
${ }^{2}$ In 2000, Agilent developed an optical switch actuated by reflection off of bubbles generated using its inkjet technology.

${ }^{3}$ In 2001, Creo Products (now a division of Kodak) developed a magnetically bent fiber optical switch based on its laser printing technology.
} 
technology is the current leader, integrated optics is advancing quickly, and photonic crystals are nearly ready to move off the drawing board. Furthermore, after the glut and confusion of the high tech boom, a few key applications for optical switches are emerging with distinct requirements. Thus, the choice of device design may depend on its intended application, and it will influence switching contrast, power requirements, insertion loss, and other aspects of device performance.

\subsubsection{Photonic crystal switches}

Photonic crystal materials are finding application in many areas, including optical switching. A photonic crystal is a lattice of dielectric material with periodicity that is designed to give desired band structures for electromagnetic (EM) radiation. This is similar to semiconductor crystal lattices which define band structures for electrons. Propagation of EM waves through the photonic crystal is only allowed for certain frequencies $\omega$ with respect to wave number $\kappa$. The gaps in frequency $\omega$ are associated with energy states that cannot exist, i.e. photonic band gaps (see Figure 2-2). Through control of the crystal lattice, the band structure of the material may be controlled to prohibit the propagation of light at specific frequencies $\omega$. Such control may be realized, for example, using LC-filled holes in the lattice. The LC refractive index is dependent on temperature and electric field and so may be modified to destroy one waveguide so that the optical signal is coupled to the desired output waveguide [16]. Such devices are currently still in the design stages, but photonic crystals offer the possibility of extremely compact optical switches on the scale of the signal wavelength. 
$\omega$

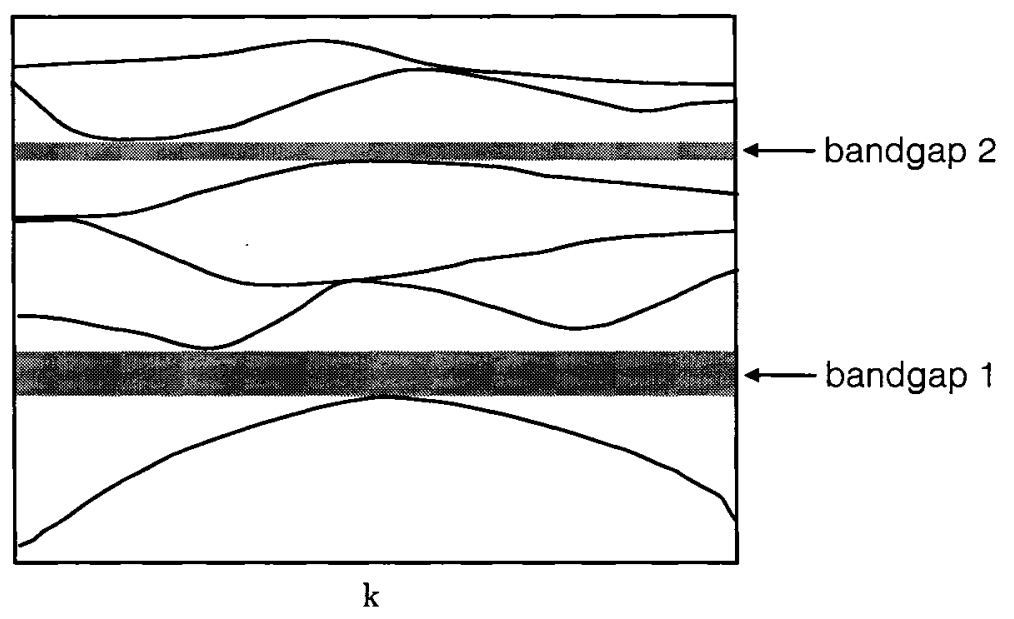

Figure 2-2 Illustration of dispersion relation (band structure), frequency $\omega$ versus wave number $k$, of photonic crystal with two bandgaps shaded in grey.

\subsubsection{MEMS switches}

MEMS are micro-structures that are fabricated using conventional micro-lithography techniques. Most MEMS optical switches are free space designs, consisting of an array of mirrors that are independently tilted to direct optical signals to desired output ports. Actuation of the mirrors is most commonly achieved electrostatically, though electromagnetic actuation offers the benefit of latching, which reduces power requirements [17]. A great deal of MEMS switch research is focused on feedback control methods, especially of the three dimensional (3D) mirrors, to deal with critical tilt angles, stability, and repeatability. A recent proposal has been the use of micromotors, which have fixed positions, so that analog control is not required [18]. Packaging is another area of research that is important for the integration of MEMS switches with other devices such as variable optical attenuators (VOAs) and demultiplexers. Being a free-space implementation, the insertion loss for MEMS switches is generally low, and the 3D design allows for large port counts. Diffraction of the unguided optical signals limits the size of the MEMS device so that port counts of greater than $\sim 1000 \times 1000$ will require a cascaded switch architecture [19]. 


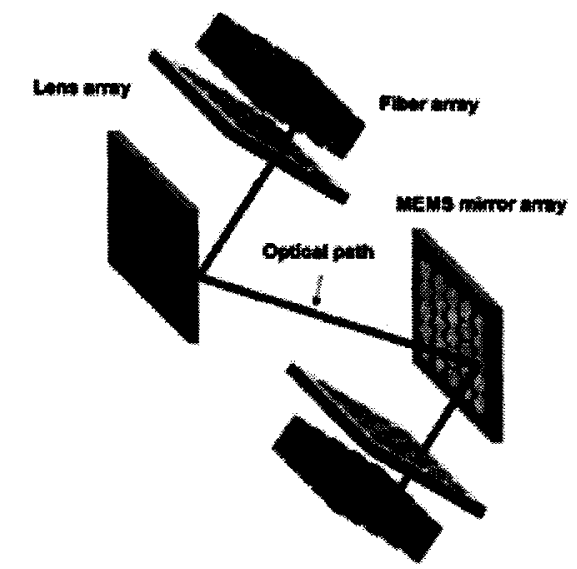

Figure 2-3 Illustration of 3D MEMS OXC with optical signals focused by a lens array onto the mirror array which is positioned to switch the signals to the desired output fibers [20].

Lately several switch designs have appeared that combine MEMS and waveguide components. For example, a wavelength selective OXC based on interferometric switching employs MEMS piston mirrors located at the output edge of a PLC to change optical path lengths and direct signals to different output ports [21]. Another design comprises waveguides that can be re-positioned to allow evanescent coupling to an adjacent waveguide, similar to a directional coupler [22]. Yet other designs involve the insertion of MEMS mirrors into the optical path of $\mathrm{Y}$ - or X-splitter switches [23, 24]. Such waveguide-based designs are much more compact than the free-space designs using mirrors, and they also switch more quickly (on the order of $\mu \mathrm{s}$ ) because of the smaller translational distances required.

Dicon Fiberoptics offers a 24x24 matrix switch with switching time of less than $35 \mathrm{~ms}$ and power consumption of less than $500 \mathrm{~mW}$. In addition to the switching matrix, commercial products are now often packaged with control system and network management cards and software, capable of supporting GMPLS. Glimmerglass offers such a 160x160 OXC with $20 \mathrm{~ms}$ switching speed and $3.6 \mathrm{~dB}$ insertion loss, and Calient offers a $256 \times 256$ OXC with switching speeds of $10 \mathrm{~ms}$ and insertion loss of $\sim 4.5$ dB. 


\subsubsection{Integrated optical switches}

Research in PLC switches is still a broad field with a great deal of variation in material systems (polymer, III-V semiconductors, silica-on-silicon), actuation mechanisms (thermo-optical, electro-optical, current injection, quantum effects), and switching mechanisms (interference-based, reflection-based, modal evolution). PLC switches offer the benefit of efficient coupling to fiber, easy integration with other components, and small footprint. However, their weakness lies in the area of scalability. The basic $1 \times 2$ or $2 \times 2$ unit must be cascaded to build larger switching fabrics. Each successive stage of switches introduces propagation losses and crosstalk.

The main focus of current optical switching research is the reduction of power, insertion loss, polarization dependence, and device size. Switching speeds vary greatly among the different actuation mechanisms, as mentioned previously. Thermo-optical actuation has provided switching speeds on the order of milliseconds. Carrier injection is limited by carrier recombination lifetimes to several nanoseconds (ns) and electrooptical actuation can reduce that time to tens of picoseconds.

A) Semiconductor Optical Amplifier: A semiconductor optical amplifier (SOA) switch is a splitter with SOA gates placed along each path. The SOAs absorb in the off state and transmit or amplify in the on state. Therefore the SOAs are used simply to block unwanted paths and transmit or provide gain to the desired paths. The loss due to the splitter is compensated by amplification in the SOA, however splitting into a large number of channels $(>4)$ may require more than one stage of SOAs for compensation. This is a drawback since noise is amplified at each SOA stage. Such a switch design is suited though for a broadcast and select architecture, where a signal is transmitted to multiple destinations, as the SOAs can ensure lossless transmission for every output.

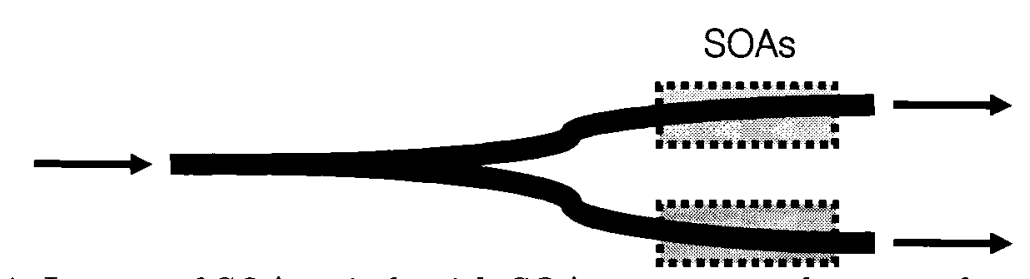

Figure 2-4 Layout of SOA switch with SOA gates on each output branch. 
Corning and IBM are developing the Optical Shared Memory Supercomputer Interconnect System (OSMOSIS) employing SOAs with a star coupler for optical switching [6]. The optical interconnect offers increased transmission bandwidth, however buffering of packets is still performed electronically. Additionally, refractive index modifications in SOAs have recently been used in interferometric switch configurations for ultrafast (sub-picosecond) switching using a pump-probe setup [25]. This implementation is rather new and the mechanisms involved are not yet fully understood.

B) Multi-mode interference: A multi-mode interference (MMI) switch generates multiple modes from the input signal through widening of the input waveguide. The modes then interfere such that the optical signal will couple to the desired output port. The benefit of the MMI design is its compactness, especially as the number of ports increases since scaling simply requires lengthening of the widened waveguide section, as opposed to cascading of devices as is required in other designs. The disadvantage, common to all interferometric designs, is the wavelength dependence and periodic nature of the output signal, which necessitates control of the switching signal and fabrication precision to optimize switching contrast.

Agashe et al. recently published an article on a 2x2 MMI switch in InGaAsP-InP with carrier injection actuation [26]. It was only $1.3 \mathrm{~mm}$ in length but required $160 \mathrm{~mW}$ of power to switch. Variation in polarization was less than $2 \mathrm{~dB}$ for the cross and bar states. A thermo-optical implementation in polymer requires less power $(22 \mathrm{~mW})$, however switching speeds are longer $(4 \mathrm{~ms})$ and the device is larger $(3.6 \mathrm{~mm})[27]$.

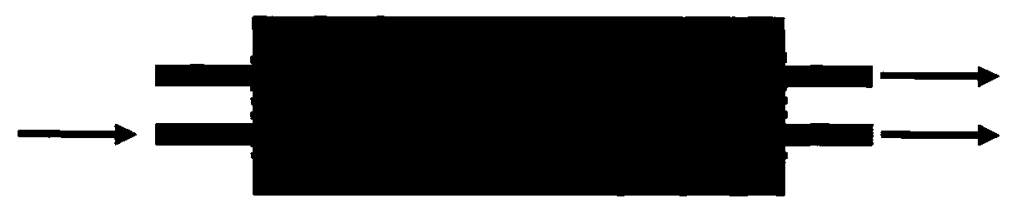

Figure 2-5 Layout of MMI switch for which an signal applied to the dashed rectangular electrode will modify the refractive index so that input light interferes to couple to the desired output port. The electrode shape is only an example.

C) Directional Coupler: A directional coupler (DC) switch has a section where the input waveguide is close enough to an adjacent waveguide that their modes are coupled, allowing optical power to move back and forth between the two waveguides. 
The optical path length of the coupled section determines to which output port the optical signal will couple. Similar to the MMI structure, a DC is sensitive to optical wavelength and the optical path length of the active region, making it very difficult to fabricate and control. In fact research into DC switches over the past few years is minimal, with the exception of some proposed designs using photonic crystals [28].

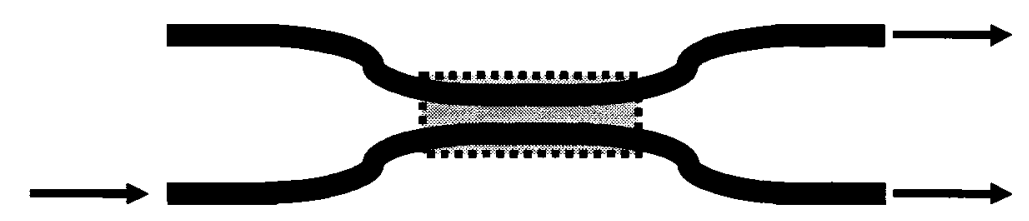

Figure 2-6 Layout of DC switch for which an signal applied to the dashed rectangle will modify the refractive index so that input light couples to the desired output port.

D) Mach-Zehnder interferometer: A Mach-Zehnder interferometer (MZI) switch splits an input signal between two paths and causes the signals in those paths to interfere such that the optical signal will couple to the desired output port. The MZI design is commonly employed and has been implemented in semiconductors [29, 30], polymers [31], high-index contrast materials [32], and photonic crystals [33]. The advantage of the MZI design is the small refractive index variation required for switching. The disadvantages again as an interferometric-based switch are precise control signal and fabrication tolerances and wavelength dependence.

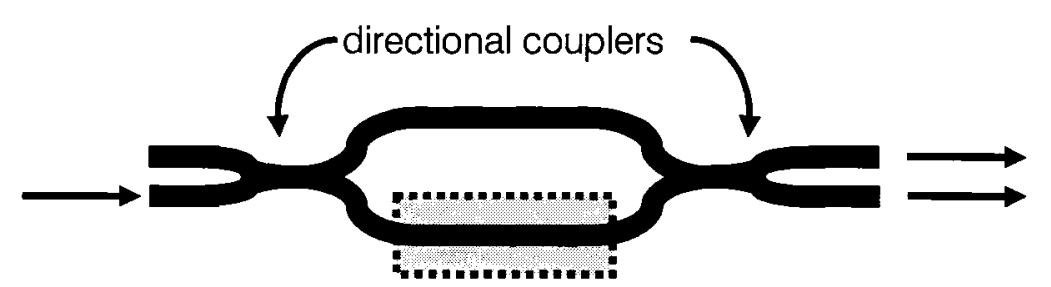

Figure 2-7 Layout of a MZI switch with an actuation element on the bottom arm to control phase so that the interference between the two arms cause the signal to couple to the desired output port.

MZI switches have been actuated by a variety of techniques, from the very common thermo-optical [31, 32], carrier injection [30], electro-optical [29], and all-optical [33] methods, to the more unconventional use of a liquid crystal cladding to effect a waveguide index modification [10]. A recent development has been the use of ring resonators coupled to the arms of a MZI as the means of inducing the phase shift [34, 
35]. By taking advantage of the small changes that can destroy resonant coupling, the required switching power is appreciably reduced; however, relying on the resonator structure results in very strong wavelength dependence.

E) Ring Resonator: A ring resonator is a ring-shaped waveguide with adjacent straight waveguides placed sufficiently close to form directional couplers that enable input and output coupling. Such a device has a resonant frequency, dependent on the ring circumference, at which the optical signal will be coupled through the ring to the second waveguide. Modification of the refractive index of the ring will change its resonance, enabling an optical signal to be switched between either the input waveguide or the adjacent output waveguide. The ring radius and the coupling coefficient between the ring and bus are designed to obtain the desired switching power and speed. Devices are very compact, with fabricated ring radius down to $5 \mu \mathrm{m}$ [36]. Switching speeds of $10.5 \mathrm{ps}$ have been achieved in a silicon ring-resonator microswitch [37]. This ring design and the resonant nature of the switching allow a very compact device to be fabricated on high index contrast materials and to require little power $(\sim 6 \mathrm{~mW})$. However, the resonant nature of the switching limits operation to a narrow wavelength band of less than $1 \mathrm{~nm}[38]$.

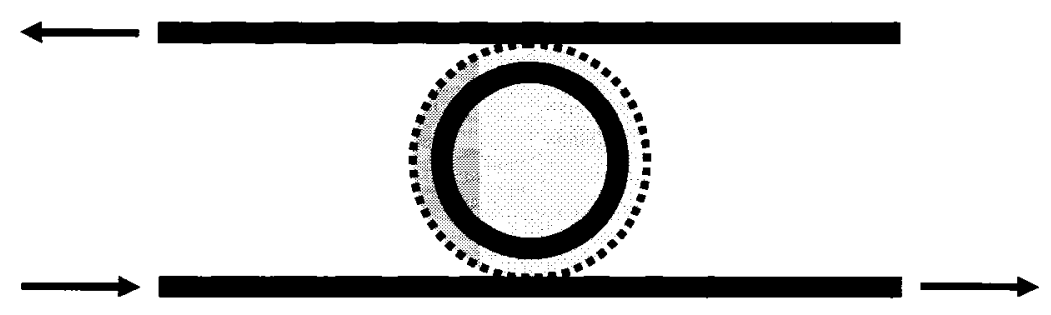

Figure 2-8 Illustration of ring resonator switch with input at bottom left and actuation signal applied to dashed circle to control resonance so that the signal is switched to either output.

SOI is also a popular material for ring resonators because the strong waveguide confinement allows for small bend radii and thus smaller rings. Tuning has been accomplished both thermo-optically [39] and all-optically [36].

F) Digital Optical Switch: Most of the PLC designs mentioned so far, however, are not digital. A digital optical switch (DOS) [40] is one that remains in the switched state above a given threshold. Compared to switches that rely on interference, DOS are 
attractive because they are wavelength independent and have relaxed bias tolerances. It is therefore important to find improved DOS designs and to optimize their performance.

The most prevalent DOS designs are $\mathrm{Y}$ - and X-splitters. Y-splitters switch by manipulation of the input mode into the desired output port by modification of the refractive index of one arm of the Y-junction. One example is the adiabatic modal evolution switch, which requires only a small index modification but a small branching angle so that the output waveguides are close enough to interact over a long propagation length. While improvements can be made by shaping the branches [41, 42], tapering electrodes and waveguides [43], and using double-etched waveguides with variable height and width [44], low crosstalk and compactness are difficult to achieve simultaneously. For devices to have better than $20 \mathrm{~dB}$ crosstalk the electrodes had to be several millimeters long and the total device length was on the order of $1 \mathrm{~cm}[43,44]$. In addition to high power consumption, the capacitance of such long electrodes can limit the switching speed even though the switching mechanism itself can be intrinsically fast.

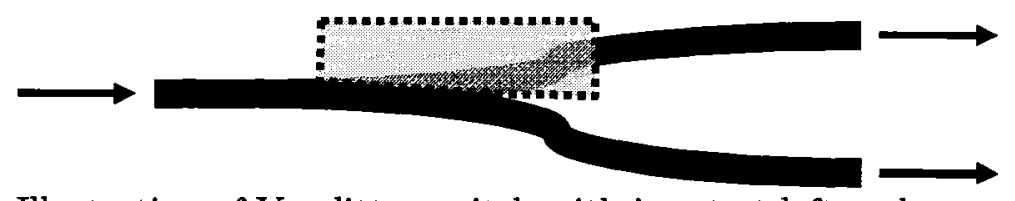

Figure 2-9 Illustration of $Y$-splitter switch with input at left and actuation signal applied to dashed rectangle to control refractive index so that the signal is switched to either output.

$\mathrm{X}$-splitters switch by total internal reflection (TIR) at the junction of two crossing waveguides, as shown in Figure 2-10. With no actuation, input light travels from the bottom left waveguide straight through the junction to the top right output. Upon actuation of the electrode, outlined by the dashed line, the input signal is reflected into the bottom right output waveguide. DOS designs using TIR have larger branching angles and shorter electrodes. However, even with improvements such as bow-tie electrodes [45], the smallest reported electrode length was $0.6 \mathrm{~mm}$ with an overall device length of $5 \mathrm{~mm}$. 
(a)

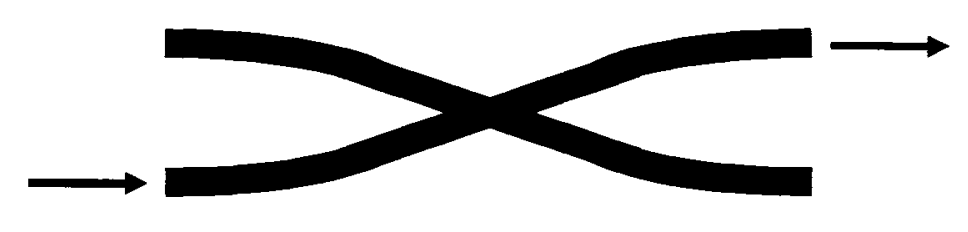

(b)

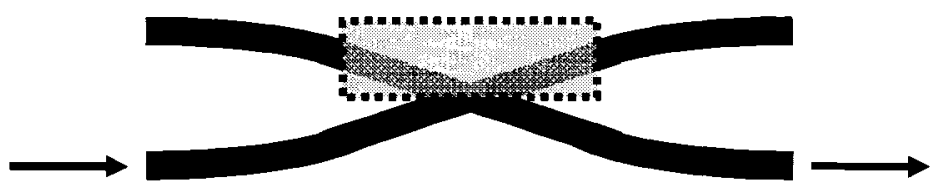

Figure 2-10 Illustration of X-splitter switch with input at left (a) switching to top output arm with no applied bias and (b) switching to bottom output arm as the input signal reflects off the edge of dashed rectangular control pad.

Further comparison, with more detailed illustrations, of the $\mathrm{X}$ - and $\mathrm{Y}$-splitters is given in Section 3.1.

G) Commercial development: Development of most waveguide-based switches is currently targeted towards the protection switching market. Lynx Photonic Networks' $8 \mathrm{x} 8$ bypass switch has the same design as NEL's, with switching speeds of $<2 \mathrm{~ms}$ and a lower insertion loss of $<1.5 \mathrm{~dB}$. Dupont currently offers an $8 \times 8$ OXC switch in polymer with switching speeds of $3 \mathrm{~ms}$ achieved thermo-optically. The insertion loss is $5 \mathrm{~dB}$ and the device dissipates $4 \mathrm{~W}$ of power. The constituent switch devices are DOSs. NTT Electronics (NEL) offers a similar product, however the implementation is a MZI design on silica-on-silicon.

Only two companies so far have targeted optical burst and packet switching. Nozomi Photonics uses $(\mathrm{Pb}, \mathrm{La})(\mathrm{Zr}, \mathrm{Ti}) \mathrm{O} 3$ (PLZT) thin-film waveguides in a DC configuration for electro-optical switching at $<6 \mathrm{~ns}$ using $10 \mathrm{~mW}$. A $2 \times 2$ configuration of such switches incurs a loss of $6 \mathrm{~dB}$. Yokogawa Electric's $1 \times 2$ switch is a TIR current injection X-splitter design fabricated on GaAs $[46,47]$. Waveguide widths vary from 4 to $8 \mu \mathrm{m}$ and branching angles vary from 4 to $8^{\circ}$. Switch rise and fall times are less than $2 \mathrm{~ns}$ and insertion loss is $10 \mathrm{~dB}$, including coupling from fiber to waveguide. With $200 \mathrm{~mA}$ applied current, switching contrast is $15 \mathrm{~dB}$. Higher extinction ratios and buffering may be achieved by cascading switches. Such a $6 \times 6$ structure has a $12 \times 1.2 \mathrm{~mm}$ footprint. These devices represent the leading edge of switching technology and have comparable performance to the RW-DOS devices developed in this thesis. 


\subsubsection{Waveguide Attenuators}

Attenuators can generally be classified into two main categories: those based on extinction, and those based on switching. Attenuation based on switching employs the switching structures described in the last section with attenuation achieved by switching the signal to an unterminated output. Attenuators based on extinction are generally more compact than those based on switching and in fact can make use of existing connection waveguides in a photonic integrated circuit. Such extinction-based attenuators are generally straight or bent waveguides, and the optical signal is attenuated through absorption or radiation loss due to reduced confinement.

A) Straight waveguides: Gemfire Corporation offers a VOA implemented as a straight waveguide in polymer and actuated using resistive heaters, with $>30 \mathrm{~dB}$ attenuation and consuming $<10 \mathrm{~mW}$ per port [48]. Attenuation is achieved through waveguide spoiling by heating the top surface of the waveguide to create an asymmetric vertical index profile. Optical power is thus attenuated through radiation loss into the substrate. Variations on such a design include widening the waveguide out to a multimode width and encouraging coupling to higher order modes through an index perturbation across the multimode region. The higher order modes are then lost when the waveguide is tapered back to a single mode width [49]. Straight waveguide designs require large refractive index modulations to induce attenuation and so are generally implemented in polymer materials, which have larger responses and thus require lower actuation powers. However, material absorption and the long-term stability of polymers is a concern and more conventional materials such as silica are preferred platforms. A silica-core/polymer-cladding hybrid modulator has been designed that attempts to exploit the best of both material systems [50]. However, polymer materials with refractive indices similar to silica are rare and the device has yet to be fabricated, although a hybrid EO polymer/sol-gel straight waveguide modulator has been fabricated [51].

B) Bent waveguides: The inclusion of a bend in a waveguide attenuator enhances loss by supplementing the mode mismatch loss incurred by a straight guide with bend losses as the waveguide effective index decreases. A polymer and silicon oxynitride 
(SiON) bent waveguide modulator has been fabricated that attenuates by decreasing core index and thus waveguide confinement [52]. However, it requires a high power of $>1 \mathrm{~W}$. Response times were also generally long, on the order of milliseconds. Corning has also developed a bent waveguide VOA in polymer that employs a vertical thermal gradient to distort the waveguide mode down into the lower cladding [53]. Power consumption was still high, on the order of several hundred milliwatts.

A modulated index profile for the lateral cladding surrounding the waveguide core has been modeled as another method of inducing attenuation through a waveguide bend. It is a novel implementation that has not yet been fabricated due to the lack of suitable polymeric material [54]. As well, fabrication would involve multiple steps of etching and deposition to obtain the required lateral profile of thermo-optical materials

At present, straight or bent waveguide attenuators are largely dominated by polymer materials. Polymers are sensitive to heat, vibration, and optical power, and are often birefringent as well. These issues, along with higher absorption and long-term instability have prevented polymers from being widely used. A more compatible material system would allow waveguide attenuators to be integrated into PLCs and even placed over existing connection waveguides, so that its footprint is minimized.

\subsection{Summary}

In the telecommunications market, increasing demand for greater network functionality is driving the development of faster optical switches for switching of data. At the moment only integrated optical switches, including the RW-DOS, have sufficient speeds on the order of several nanoseconds for burst or packet switching. Straight or bent waveguide based attenuators may beneficially be integrated with waveguide-based switch devices. 


\section{Chapter 3}

\section{RECONFIGURABLE WAVEGUIDE DIGITAL OPTICAL SWITCH}

\subsection{Device Design}

\subsubsection{Effective Index Method}

Two-dimensional beam propagation method (BPM) simulations using the effective index approximation were used to design the RW-DOS devices. The effective index method comprises representing a multi-layer structure with an effective refractive index. The effective indices were calculated using MENU, a mode solver written by A. Dêlage at IMS, NRC, by solving the modal index of the equivalent slab waveguides. In the 2D-BPM simulations, the waveguides are represented by a ridge effective index $\mathrm{n}_{\text {ridge }}$ surrounded by a slab effective index $\mathrm{n}_{\text {slab }}$, as illustrated in Figure $3-1$. This model describes a sharp refractive index step and does not include the effects of lateral diffusion of carriers, as is explained later. A waveguide mode effective index $\mathrm{n}_{\text {mode }}$ can subsequently be calculated as the modal index of an equivalent slab waveguide with a core index of $n_{\text {ridge }}$ and a cladding index of $n_{\text {slab }}$. 


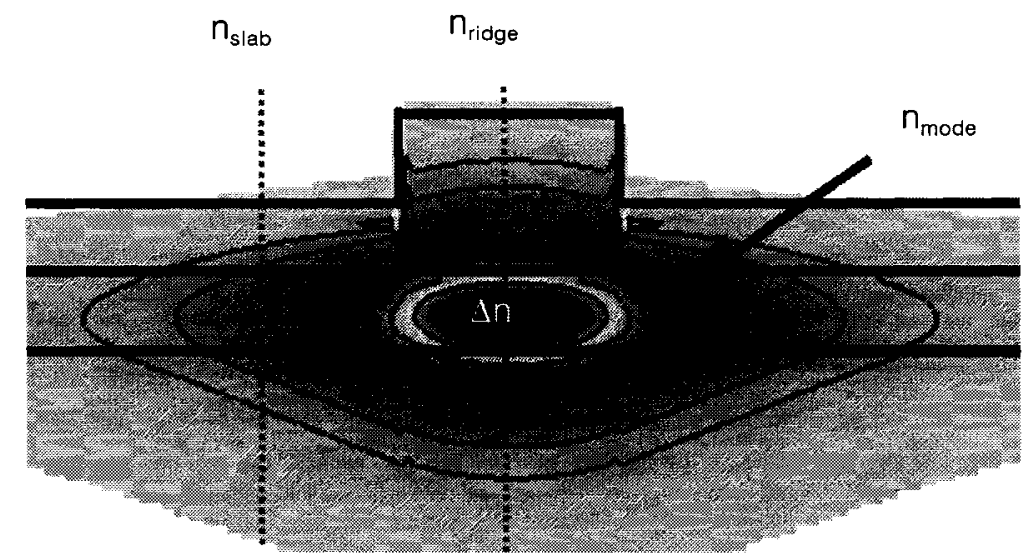

Figure 3-1 Illustration of ridge, slab, and mode effective refractive indices.

Only changes in $n_{\text {mode }}$ may be experimentally measured. To relate these to modeled changes in material refractive index $\Delta \mathrm{n}$, a series of calculations were performed in MENU to fit and extract functions relating $\Delta \mathrm{n}, \Delta \mathrm{n}_{\text {ridge }}$, and $\Delta \mathrm{n}_{\text {mode }} \cdot \Delta \mathrm{n}_{\text {ridge }}$ is linearly proportional to $\Delta \mathrm{n}$, though 20 to $30 \%$ smaller. $\Delta \mathrm{n}_{\text {mode }}$ is a nonlinear function of $\Delta \mathrm{n}$.

\subsubsection{Device Configuration and Operation}

The configuration of the proposed reconfigurable waveguide (RW)-DOS is shown in Figure 3-2 (a) with waveguide ridges illustrated by the shaded regions. It is comprised of a straight input waveguide, diverging into two S-bend waveguides with straight waveguides at their output ends. Without an applied switching signal, the input power is split evenly between the two output branches. The inner edge of each electrode follows the curvature of the opposite waveguide but is displaced outwards by a $1 \mu \mathrm{m}$ gap to allow for carrier diffusion. Note that the electrodes are only in electrical contact with the device where they overlap the waveguide ridges. With no applied bias, the input optical power is split evenly between the two output branches.

A negative index change, $\Delta \mathrm{n}$, under the biased electrode causes the electrode edge to form the outer boundary of the unbiased waveguide. Figure 3-2 (b) shows the effective index distribution in the $\mathrm{Y}$-junction and surrounding slab waveguide when one electrode is forward biased, decreasing the effective index under the electrode. When the induced index change $\Delta \mathrm{n}$ is approximately equal to the effective index step $\delta n_{\text {eff }}$ 
between the ridge waveguide and the adjacent slab waveguide region, the input waveguide and unbiased branch will effectively form a single curved waveguide guiding the light through the unbiased arm as shown in Figure 3-2 (b). Thus the input signal does not pass through or suffer additional losses from the carrier injection region. In fact, the total optical power transmitted through the device may increase with biasing due to decreased scattering losses at the $\mathrm{Y}$-junction, as will be demonstrated later.

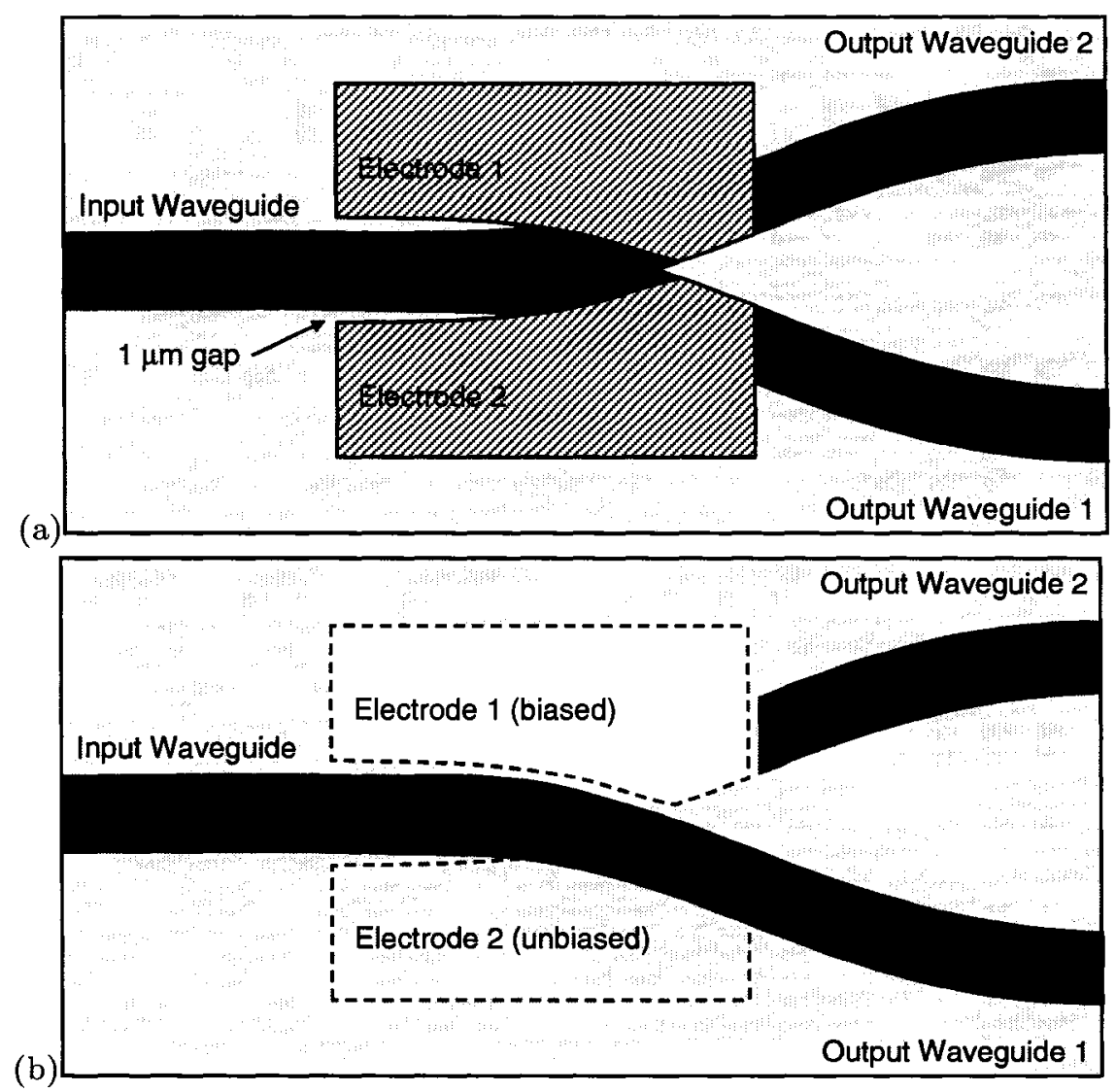

Figure 3-2 (a) Configuration of the proposed DOS Y-junction switch with input at the left and outputs at the right and (b) Effective waveguide configuration when the index under the biased electrode matches the refractive index of the surrounding slab region.

As mentioned previously, index modulation may be accomplished through a variety of methods, including electrical carrier injection, the quick establishment of a thermal gradient, the quantum confined stark effect (QCSE) in quantum wells, or nonlinear effects. However, a negative index step, as provided by carrier injection, is required for RW-DOS operation. 
A positive index step, as provided by the thermo-optical effect, will also facilitate switching in the RW-DOS structure, though through a different mechanism. An increase in effective refractive index in the biased arm of the y-splitter will refract the input signal into that arm. Unlike operation of the RW-DOS using a negative index change, refraction-based switching results in the existence of an optimum branching angle, related to the angle of refraction, at which the DOS will switch a maximum power. Also, since the input signal is switched through the biased arm, it now passes through the index modified region and could therefore suffer additional losses. Thus although it is possible to operate the RW-DOS with a positive index change, a negative index change is preferred.

\subsubsection{Advantages of RW-DOS Design}

Comparative device simulations show that this DOS design is superior to many previous switch designs reported in the literature in terms of power requirements and size. Switching performance was simulated and compared for the proposed reconfigurable waveguide digital optical switch (RW-DOS), an adiabatic mode coupling switch, and a bow-tie total internal reflection (TIR) switch. For the modeling of all three of the following examples, the ridge width was 3 micrometers and the effective indices were $n_{\text {ridge }}=3.2745$ and $n_{\text {slab }}=3.2709$, corresponding to the same layer structure as used in the fabricated RW-DOS devices (described in Section 3.2). The remaining switch design parameters were chosen to keep the insertion loss below $1 \mathrm{~dB}$, achieve at least $20 \mathrm{~dB}$ switch contrast ratio, and have comparable Y-branch angles as in our RWDOS design (i.e. $\sim 0.9^{\circ}$ ). The light was assumed to be TE polarized in all cases. Simulations were done using a two dimensional beam propagation method (BPM) model and the effective index approximation.

A common DOS design is the adiabatic mode evolution Y-branch switch. Such switch designs are usually characterized by long active electrode lengths (e.g. $30 \mathrm{~mm}$ ), small branching angles of less than $0.2^{\circ}$ (e.g. $0.048^{\circ}$ ), and "high drive voltage (or power)" [55]. The large device size is due both to the long electrode lengths as well as the small branching angles, which result in longer devices because of the need to establish sufficient waveguide separation $(\sim 20 \mu \mathrm{m})$ at the outputs. 
In an attempt to reduce drive voltage while maintaining acceptable crosstalk, several variations to the conventional $\mathrm{Y}$-branch have been suggested. The arms of the conventional Y-branch are composed of two linearly diverging waveguides. One variation comprises arms formed by tapered waveguides such as in the linear and shaped DOS designs. Another variation comprises a single large taper at the branching point [56]. In our simulations we used a linear DOS design from reference [55], illustrated in Figure 3-3 (a).

A total internal reflection (TIR) DOS design is the X-branch switch [57] shown in Figure 3-3 (b). This switch design is usually characterized by large index modulation required for switching $(-0.01)$. X-branch switches differ from Y-branch switches in that in the "off" state of the X-branch switch light is directed to one output arm rather than split between the two output arms of a Y-branch switch.

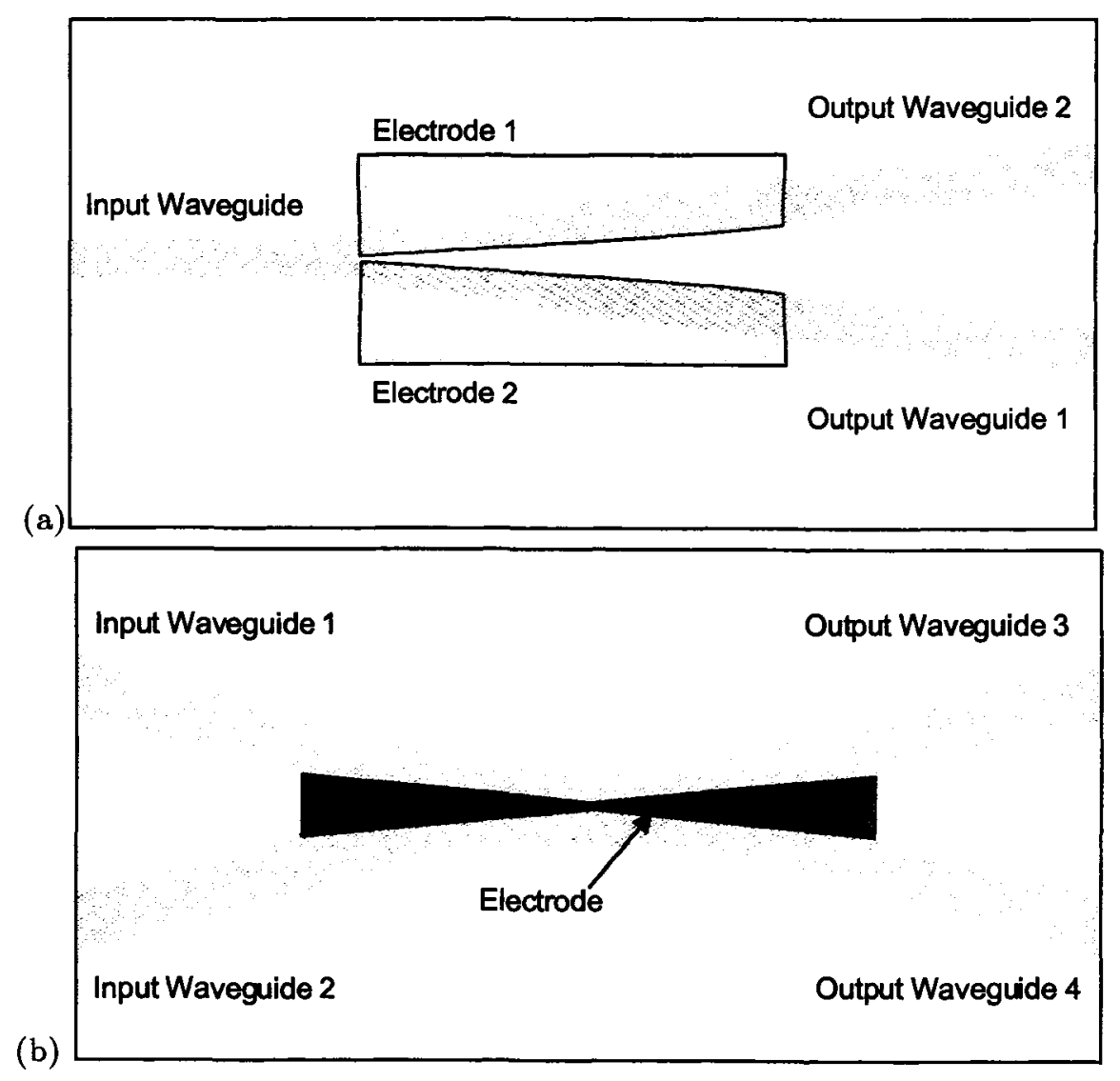

Figure 3-3 (a) Adiabatic mode evolution Y-branch switch design with tapered waveguides and (b) a TIR X-branch switch with bowtie shape electrodes, designed to allow larger branching angles [45]. 
The best way to compare different DOS designs is to examine the variation of their switching contrast ratio with $\Delta \mathrm{n} \cdot \mathrm{L}$, the product of induced index change $\Delta \mathrm{n}$ and electrode length L. For a given ridge width, this optical path length change is proportional to the injected current, and hence the required switching power. The calculated contrast ratio is defined as ten times the logarithm of the ratio of the powers from the two switch output ports. The calculated contrast ratios versus $\Delta \mathrm{n} \cdot \mathrm{L}$ are shown in Figure 3-4.

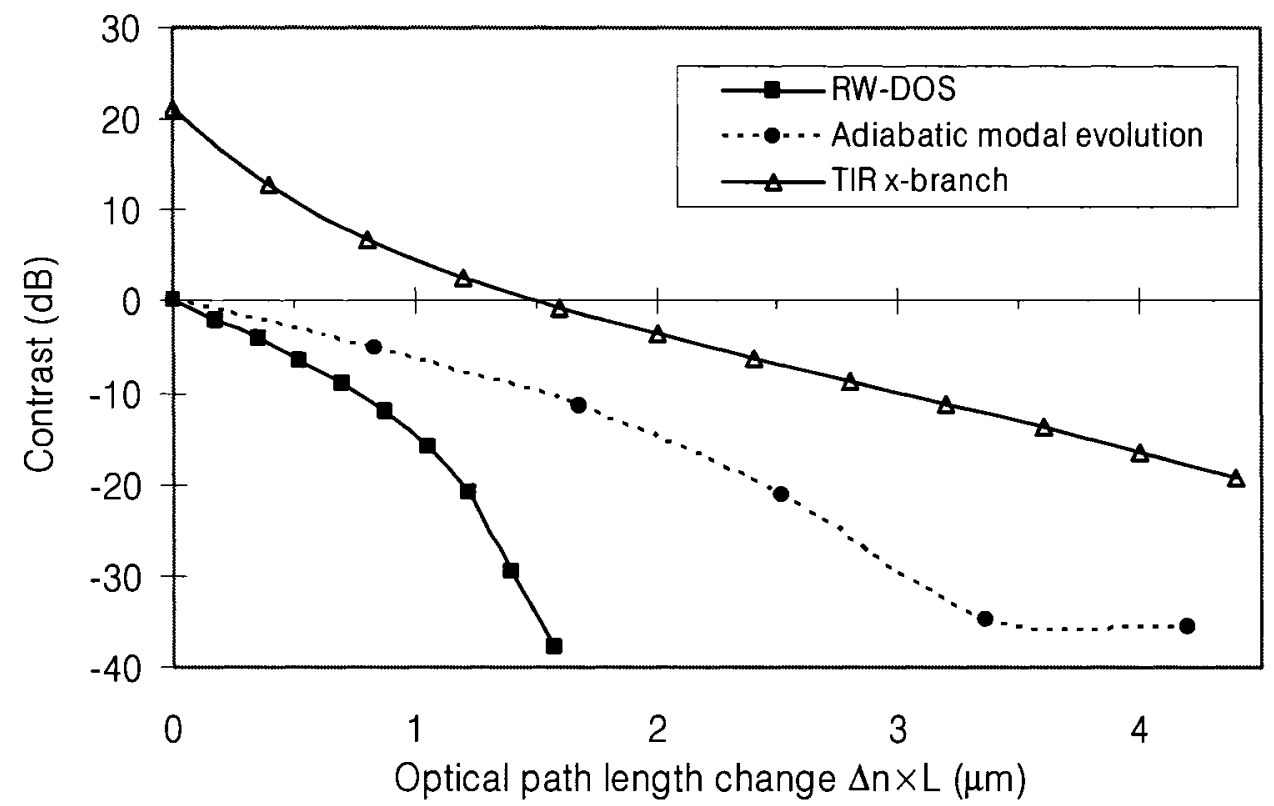

Figure 3-4 Switching contrast in $\mathrm{dB}$ between arms of RW-DOS, modal evolution, and TIR switches versus product of index modulation and electrode length.

The proposed RW-DOS switch (solid squares) clearly achieves the largest contrast at the smallest index length product. The TIR X-branch switch with a bow-tie electrode (Figure 3-3 (b)) requires a large index modulation to switch because unlike all other designs, at zero index modulation the contrast is near maximum rather than zero. Our simulations show that the $0.1^{\circ}$ adiabatic mode evolution DOS achieves high extinction $(>20 \mathrm{~dB})$ at low index modulations on the order of $\Delta \mathrm{n} \sim 0.001$. However, because the angle is small and the electrodes are very long, the required switching power (or $\Delta \mathrm{n} \cdot \mathrm{L}$ ) is higher, and the device is much longer than the RW-DOS. Increasing the Y-branch angle to $0.9^{\circ}$ in the adiabatic switch causes the switch contrast ratio to decrease by an order of magnitude for any given $\Delta \mathrm{n} \cdot \mathrm{L}$. 


\subsection{Device Actuation}

As mentioned in the Introduction above, actuation of the RW-DOS may be accomplished through a variety of methods. The bulk of the work in this thesis centers on actuation by carrier injection because it provides the necessary negative refractive index modification, and because of its adequate response speed and wavelength and polarization independence. However, work is also done to increase response speed through electro-optical actuation. Thermal gradient actuation is also explored because although it induces a positive index change, it provides the local index gradient required for switching of the RW-DOS device very quickly, resulting in fast thermal switching.

\subsubsection{Carrier Injection}

Electrical carrier injection offers the benefits of wavelength and polarization insensitivity. Carriers injected into the waveguide core layer modify the refractive index through free carrier absorption, bandgap shrinkage, and bandfilling. Figure 3-5 shows the total calculated index modulation $\Delta \mathrm{n}$ (thick solid line) due to carrier injection for the InGaAsP alloy with bandgap $\mathrm{Q}=1.3 \mu \mathrm{m}$, at an operating wavelength $\lambda=1.55 \mu \mathrm{m}$. The model for free carrier absorption and values of carrier mobility and effective mass are taken from Weber [58]. The model for bandfilling is taken from Bennett et al. [59], and the model for bandgap shrinkage is taken from Botteldooren and Baets [60]. The concentration of holes is assumed to be equal to the concentration of electrons, $\mathrm{N}=\mathrm{P}$. Also plotted in Figure 3-5 are the individual refractive index changes due to each of the three contributions to carrier induced index modulation. 


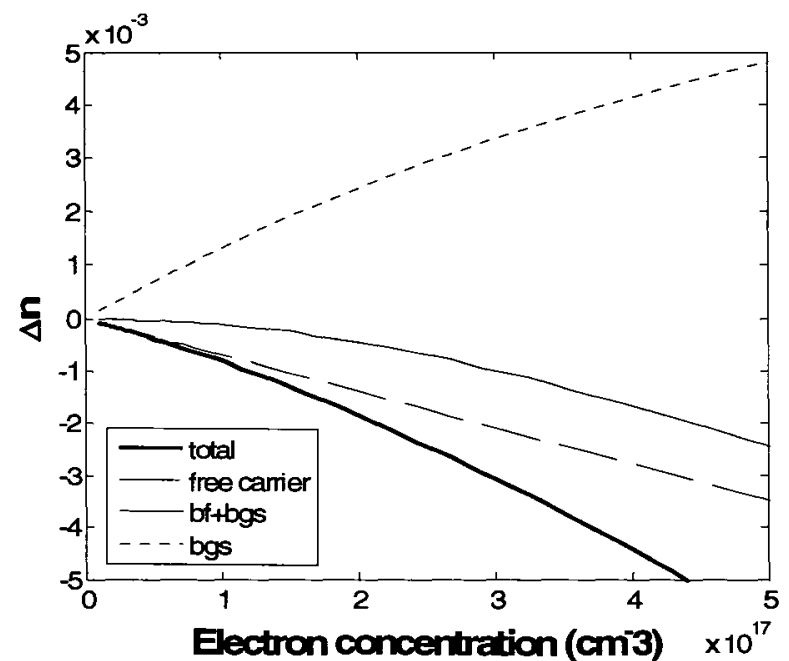

Figure 3-5 Model of refractive index change as a function of carrier concentration for $\mathrm{Q}=1.3 \mu \mathrm{m}$ InGaAsP alloy according to [58-60]. The contributions of free carrier absorption, band-gap shrinkage (bgs), and bandfilling after bandgap shrinkage (bf+bgs), are shown in dashed, dotted, and solid lines respectively.

Free carrier absorption, also termed the plasma effect, modifies refractive index through absorption of photons that excite free carriers to higher energy states through scattering with the crystal lattice, defects, or impurities. The resulting decrease in refractive index $\Delta n_{\text {fca }}$ can be obtained from modeling of the electric field induced polarization density. This was calculated by Henry et al. [61] to be:

$$
\Delta n_{f c}=-\frac{q^{2} \lambda^{2}}{8 \pi^{2} c^{2} \varepsilon_{0} n}\left(\frac{N}{m_{e}}+\frac{P}{m_{h}}\right)
$$

where $q=1.602 \times 10^{-19}$ Coulombs (C) is the electron charge, $c=2.998 \times 10^{8} \mathrm{~m} / \mathrm{s}$ is the speed of light, $\varepsilon_{0}=8.854 \times 10^{-12} \mathrm{C}^{2} / \mathrm{J} \cdot \mathrm{m}$ is the permittivity of free space, $n$ is refractive index, and $m_{e}\left(m_{h}\right)$ and $N(P)$ are the electron (hole) mass in kg and density in $\mathrm{m}^{-3}$ respectively.

Alternatively, the refractive index change can be determined through Kramers-Krönig transformation of measured absorption curves. The Kramers-Krönig relations relate the real $\varepsilon_{\mathrm{r}}$ and imaginary $\varepsilon_{\mathrm{i}}$ terms of material permittivity as a function of frequency $\omega$, and therefore the refractive index and absorption: 


$$
\begin{aligned}
& \varepsilon_{r}(\omega)=\frac{2}{\pi} P \int \frac{\omega^{\prime} \varepsilon_{i}\left(\omega^{\prime}\right)}{\omega^{\prime 2}-\omega^{2}} d \omega^{\prime} \\
& \varepsilon_{i}(\omega)=-\frac{2 \omega}{\pi} P \int \frac{\varepsilon_{r}\left(\omega^{\prime}\right)}{\omega^{\prime 2}-\omega^{2}} d \omega^{\prime}
\end{aligned}
$$

where $P$ indicates the principle value ${ }^{4}$ of the integral.

Weber [58] took the Kramers-Krönig transformation of absorption measurements for $\mathrm{p}$ type InGaAsP to determine the index change due to free hole carriers. The index change resulting from free carrier absorption that is plotted with a dashed line in Figure 3-5 is calculated according to Weber [58].

Bandgap shrinkage and bandfilling are determined through modeling of how the carrier density modifies the absorption spectrum. Starting with the simplest model for a direct-gap semiconductor assuming parabolic bands, the absorption $\alpha_{0}$ is [59]:

$$
\alpha_{0}=C * \frac{\sqrt{E-E_{g}}}{E}
$$

where $C$ is a constant specific to the material, $E$ is the photon energy, and $E_{g}$ is the material bandgap energy.

Bandgap shrinkage $\Delta E_{g}$ occurs as injected carriers occupying states at the top and bottom respectively of the valence and conduction bands screen each other out. According to [60], the bandgap shrinkage is given by:

$$
\Delta E_{g}=\frac{e^{2}}{2 \pi^{2}} \int \frac{1}{4 \pi \varepsilon \varepsilon_{0}}\left[\frac{1}{1+\exp \left\{\left[E(q)-E_{f}\right] / k_{b} T\right\}}\right] \frac{1}{q^{2}} d q^{3} \hbar
$$

where $E_{f}$ is the Fermi level, $T$ is the temperature, and $E(q)=\hbar^{2} q^{2} / 2 m$. Bandgap shrinkage increases refractive index and is plotted with a dotted line in Figure 3-5.

\footnotetext{
${ }^{4}$ The principle value is the integration over the integration range except at the function singularity
} 
Bandfilling, or the Burstein-Moss effect, is due to the filling of energy states at the top of the valence and bottom of the conduction band. The absorption is modified by a term representing the probability $f$ that a state $E$ is unavailable:

$$
f_{v}\left(E_{a}\right)-f_{c}\left(E_{b}\right)
$$

where $f_{v}\left(f_{c}\right)$ is the probability that an energy level $E_{a}\left(E_{b}\right)$ in the valence (conduction) band is occupied by an electron.

This bandfilling term is applied to the absorption spectrum after it has undergone bandgap shrinkage. Thus the effects of both bandgap shrinkage and bandfilling are modeled as:

$$
\alpha(E)=C * \frac{\sqrt{E-E_{g}-\Delta E_{g}}}{E}\left[f_{v}\left(E_{a}\right)-f_{c}\left(E_{b}\right)\right]
$$

The index change $\Delta \mathrm{n}_{\mathrm{bgs}+\mathrm{bf}}$ due to bandgap shrinkage and bandfilling is then determined by applying Kramers-Krönig transformation to the total change in absorption $\left(\Delta \alpha=\alpha-\alpha_{0}\right)$

$$
\Delta n_{b g s+b f}=\frac{\hbar c}{\pi|e|} P \int_{0}^{\infty} \frac{\Delta \alpha}{x^{2}-E^{2}} d x
$$

where $P$ indicates the principle value ${ }^{5}$ of the integral.

These two contributions of bandgap shrinkage and bandfilling have opposite effects and largely cancel each other, especially at small carrier concentrations, as is illustrated by the thin solid line in Figure 3-5.

The carrier induced index modification due to all three contributions is then the sum of equations (3-1) and (3-8). This is plotted with a thick solid line in Figure 3-5.

\footnotetext{
${ }^{5}$ The principle value is the integration over the integration range except at the function singularity
} 


\subsubsection{Quantum Confined Stark Effect}

Index modulations can also be applied through the quantum confined stark effect (QCSE) where a reverse bias voltage applied perpendicular to the QW layers tilts the quantum well band structure (Figure 3-6 (a)) so that the absorption spectrum redshifts (Figure 3-6 (b)). This results, through the Kramers-Krönig relation, in a positive refractive index modulation. The QCSE is faster than carrier injection since only the $\sim 10$ ps RC time constant of the device limits how fast the electric field can be set up. Thus, the QW devices operating in reverse bias are expected to operate at speeds on the order of tens of picoseconds.

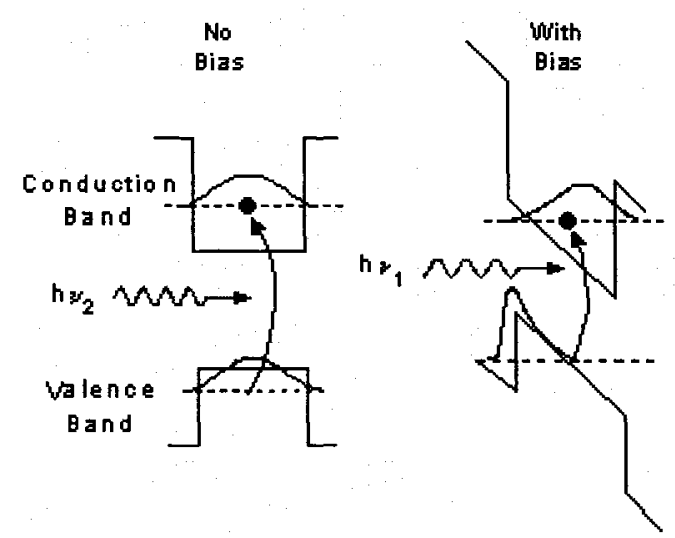

(a)

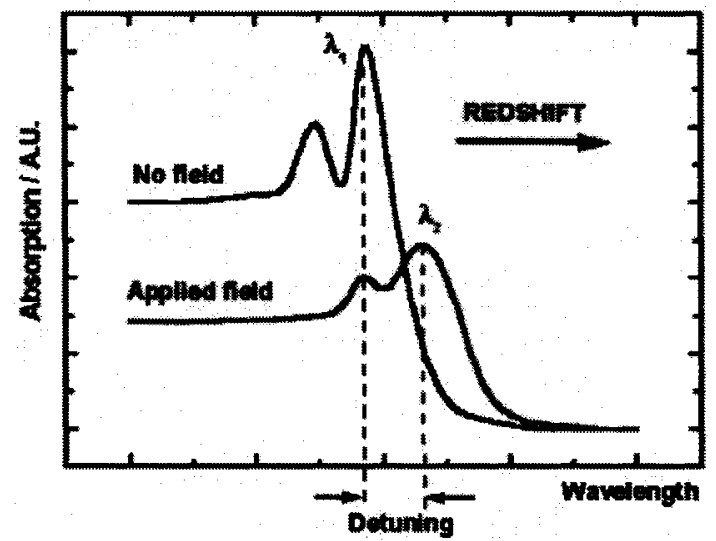

(b)

Figure 3-6 Illustration of tilting of the bands with applied field [62] (a) resulting in a redshift [63] (b) of the exciton transition peak in the absorption spectrum.

Similar to carrier induced refractive index modification, the magnitude of index change is larger at wavelengths closer to the material bandgap, but so is absorption due to the Urbach tail. To characterize the devices at $1550 \mathrm{~nm}$, the bandgap of the material was designed with this tradeoff in mind. Additionally, in QW superlattices, valence band energy is split into heavy and light holes. The heavy holes couple preferentially to TE polarizations resulting in a polarization dependent absorption spectra [64]. The wells are often strained to compensate for this polarization dependence; however, another method of compensation is to operate at a wavelength that is far from the band edge, where the polarization dependence is smaller [64]. According to literature [64, 65], a wavelength detuning of $140 \mathrm{~nm}$ is a good compromise between index change and absorption. 
The Stark shift depends on the depth and width of the quantum wells and the applied voltage. Common methods of enhancing refractive index modification while maintaining low absorption are the use of symmetric and asymmetric coupled quantum wells. However, these require alternating very thin layers of alloys having large differences in bandgap. Stepped QW, on the other hand, require neither the thin layers nor the diverse alloy compositions. A three-step QW structure was reported to enhance the electro-optical effect by threefold [65]. Finally, quantum well superlattices may be designed so that carrier injection induces negative changes in refractive index [66].

\subsubsection{Thermal Gradient}

Another method of fast index modulation is, through the thermo-optical effect, the establishment of a thermal gradient. Figure 3-7 shows the calculated temperature profiles at different times near a $5 \mu \mathrm{m}$ wide heat source embedded in the InGaAsP waveguide core [67]. After $500 \mathrm{~ns}$, a steep thermal gradient is already established at the edge of the heat source, and this local gradient remains unchanged as the peak temperature approaches steady state over about one millisecond. After the heat source is switched off, the gradient decays within a few hundred nanoseconds. The conclusion is that a switch using a local thermal gradient should operate several orders of magnitude faster than a conventional thermo-optical device.

Local heating under the electrodes is induced by current injection under forward bias, resulting in a positive index change. This effect is present in all carrier injection actuated devices. Through appropriate design of layer structure to reduce resistivity, heating can be minimized and the thermally induced index change made small in comparison with carrier induced index change. 


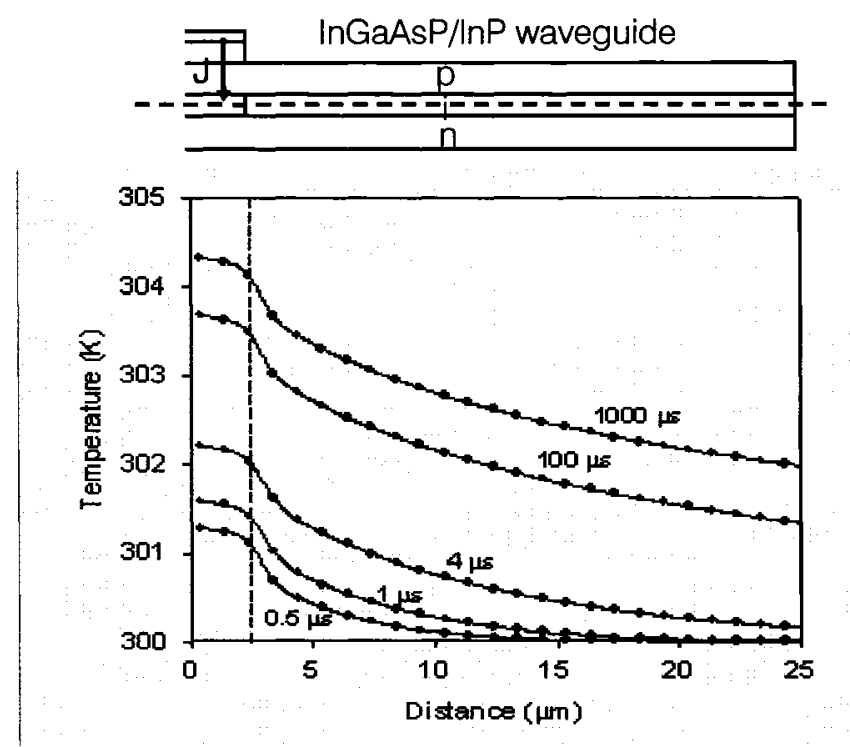

Figure 3-7 Calculated temperature variation with horizontal (in the wafer plane indicated by dashed line) distance in an InGaAsP/InP waveguide with a $5 \mu \mathrm{m}$ wide heat source (centered at $\mathrm{x}=0 \mu \mathrm{m}$ ) embedded in the InGaAsP core layer, at different times after the heat source is turned on. The dashed line indicates the edge of the heat generating region [67].

Alternatively, the layer structure may be designed to exploit the thermal gradient effect for optical switching. Such a switch was demonstrated with InGaAsP/InP waveguides. As with most thermo-optical switches, the thermal response time was approximately one millisecond. However, switching in this device does not depend on the absolute temperature, but on the thermal gradient across the waveguide. A simple analysis of the thermal diffusion equations suggests that the thermal gradient over a distance $x$ should establish itself within a response time of $\tau \sim x^{2} / \kappa$, where $\kappa$ is the thermal diffusion coefficient. For InP, $\tau$ is approximately 100 nanoseconds for $x=2$ $\mu \mathrm{m}$ [67]. Furthermore, once established this local thermal gradient is independent of time, although the peak temperature continues to rise. This analysis is confirmed by a thermal simulation of the switch waveguide after a bias step, using a three-dimensional transmission line matrix solution of the heat flow equations in a structure corresponding to fabricated devices [67].

The measured switching response for the biased and unbiased arms is shown in Figure $3-8$. Light from a broad band EDFA source $(\lambda=1530-1560 \mathrm{~nm})$ is coupled into the input waveguide. The light is directed into the biased arm, consistent with the 
positive index change expected for the thermo-optical effect in InGaAsP. A switching contrast ratio of better than $10 \mathrm{~dB}$ was achieved. The measured contrast ratio may be degraded by a second leaky mode supported in this heterostructure waveguide. The response times are about 500 nanoseconds, in qualitative agreement with the thermal simulations. These results confirm that switching is a result of the thermal gradient across the waveguide, which refracts light into the biased arm.

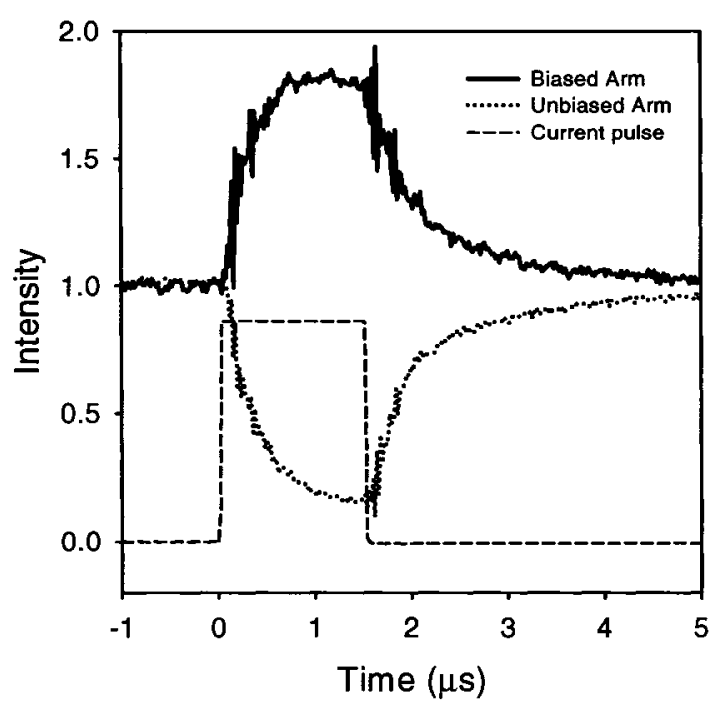

(a)

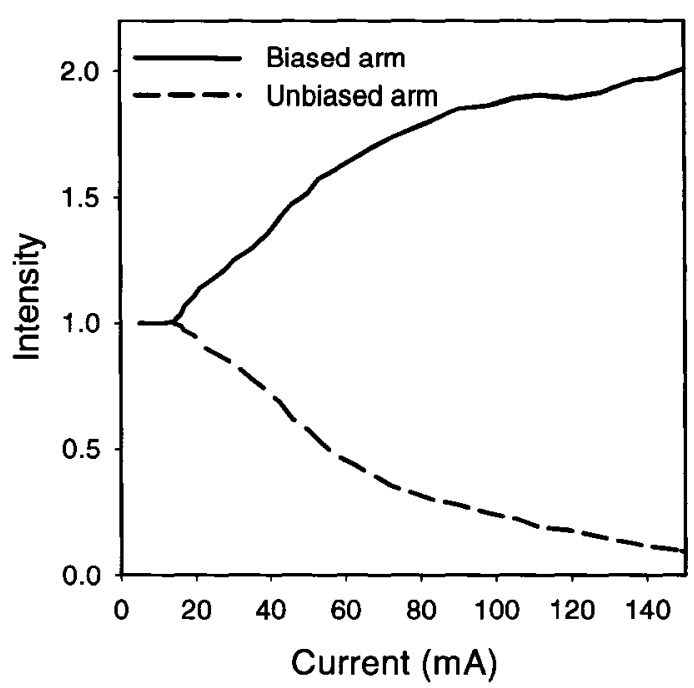

(b)

Figure 3-8 Measured output intensities vs. time (a) and peak current (b) for the biased and unbiased arms of the DOS switch for a $1.5 \mu$ s applied current pulse [67].

\subsection{Device Structure}

The RW-DOS switches were initially fabricated on InGaAsP/InP wafers with a layer structure as shown in Figure 3-9. The waveguide heterostructure was grown on an InP substrate by chemical beam epitaxy (CBE) and has a $0.5 \mu \mathrm{m}$ thick undoped InGaAsP $(\mathrm{Q}=1.3 \mu \mathrm{m})$ waveguide core. The core is surrounded by $p$ and $n$ doped upper and lower InP cladding layers, separated from the core by layers of intrinsic InP that prevent diffusion of dopants from the cladding layers into the core. An InGaAsP etch 
stop defines the height of the ridge and a cap layer of highly doped p-InGaAs provides contact for the metal electrodes deposited on top of the ridges.

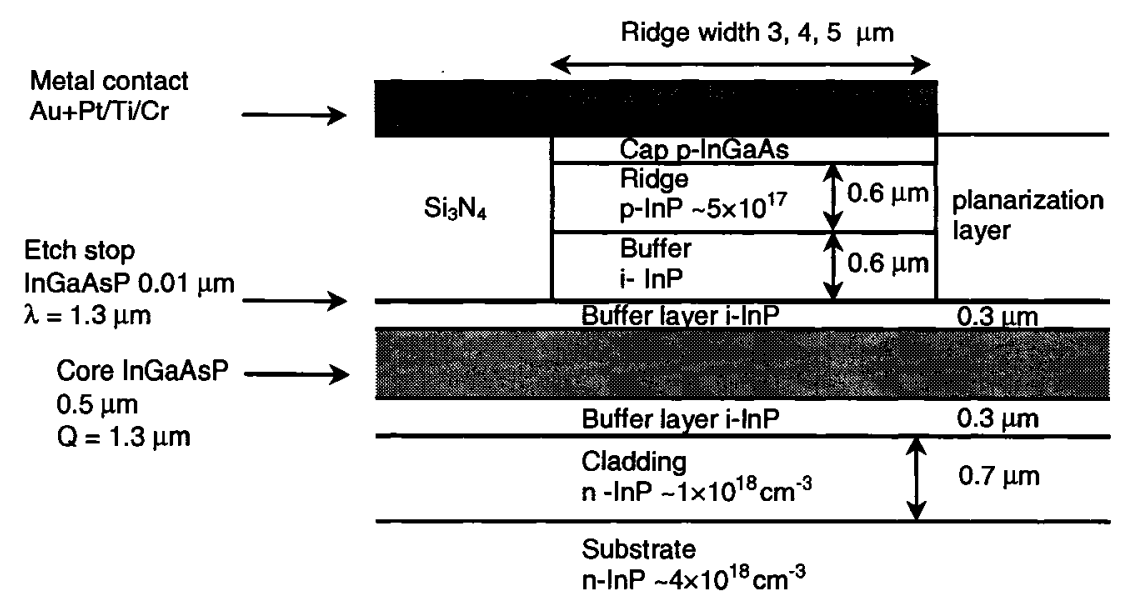

Figure 3-9 Schematic diagram illustrating the layer structure of the CBE grown InGaAsP/InP wafers.

The Y-junction was defined by etched ridges with widths of $3 \mu \mathrm{m}, 4 \mu \mathrm{m}$, and $5 \mu \mathrm{m}$. Each device had electrical contacts on each branch as shown in Figure 3-2. In our standard process, oxygen ion implantation was used to electrically isolate the contacts, which were separated by gaps of $0.5 \mu \mathrm{m}$ to $2.0 \mu \mathrm{m}$ at the Y-junction vertex. The electrode lengths ranged from $300 \mu \mathrm{m}$ to $500 \mu \mathrm{m}$ on different devices, and the switches had branching angles ranging from $0.9^{\circ}$ to $2.22^{\circ}$. For all devices, the overall length was $1.4 \mathrm{~mm}$.

A subsequent set of devices was fabricated on wafers with a thicker core $(0.6 \mu \mathrm{m})$ to increase the height of the optical mode making it more symmetric and improving coupling to and from fiber. The cores consisted of undoped InGaAsP of bandgaps 1.03 $\mathrm{eV}, 0.95 \mathrm{eV}$, and $0.88 \mathrm{eV}(\mathrm{Q}=1.2 \mu \mathrm{m}, 1.3 \mu \mathrm{m}$, and $1.4 \mu \mathrm{m}$ in that order $)$, and they were grown on the $\mathrm{InP}$ substrate by metal-organic chemical vapour deposition (MOCVD). Due to an error during growth, the undoped InP layer in the waveguide ridge between the etch stop and the upper p-doped cladding was not included. This likely contributed to the observed reduction in resistivity but had a negligible effect on waveguide mode profile. This layer structure is shown in Figure 3-10. 


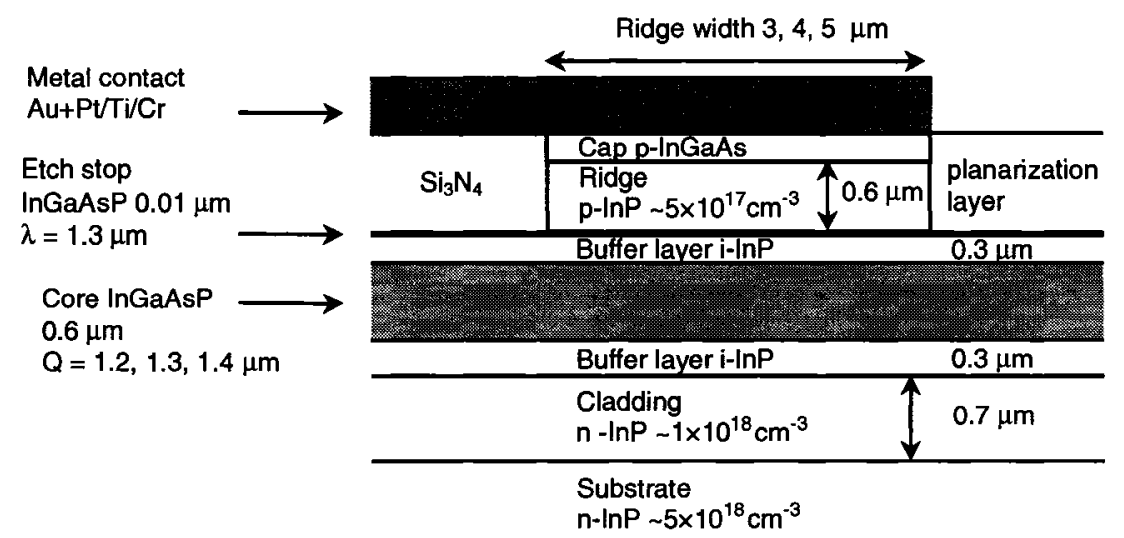

Figure 3-10 Schematic diagram illustrating the layer structure of the MOCVD grown InGaAsP/InP wafers.

The layer structures were identical for the three wafers, except for the composition of the core alloy. However, because the quaternaries have different refractive indices $(\mathbf{n}=$ $3.32,3.37,3.42$ for $\mathrm{Q}=1.2 \mu \mathrm{m}, 1.3 \mu \mathrm{m}$, and $1.4 \mu \mathrm{m}$ InGaAsP alloy respectively [58]), this resulted in different ridge and adjacent slab effective indices. More significantly, the index steps between ridge and slab regions were not uniform. The larger refractive index of the $\mathrm{Q}=1.4 \mu \mathrm{m}$ alloy resulted in waveguides with the lowest confinement. The index step was 0.0039 for the $\mathrm{Q}=1.2 \mu \mathrm{m}$ alloy, 0.0025 for the $\mathrm{Q}=1.3 \mu \mathrm{m}$ alloy, and 0.0017 for the $\mathrm{Q}=1.4 \mu \mathrm{m}$ alloy.

The difference in ridge-slab index contrast complicates a comparison of switching performance among the different core alloys fabricated on these MOCVD grown wafers. Performance is affected not only by the difference in carrier injection induced refractive index, but also by the difference in waveguide confinement. To isolate the contribution of InGaAsP alloy core to the performance of the switches, the surrounding layers were redesigned to compensate for this difference in core index. Subsequently, a final set of wafers were designed such that the lateral waveguide contrast in TE polarization is the same among the three $\operatorname{InGaAsP}$ core alloys $(\mathrm{Q}=1.2$, $1.3,1.4 \mu \mathrm{m})$. Specifically, they were designed to have $\Delta \mathrm{n}_{\mathrm{TE}}$ approximately equal to 0.0025 , as in the $\mathrm{Q}=1.3 \mu \mathrm{m}$ alloy of Figure 3-10. The designed values are shown in the table below. The $\Delta \mathrm{n}_{\mathrm{TM}}$ of the wafers could not be made equal and so varies between the three alloys, with the $\mathrm{Q}=1.4 \mu \mathrm{m}$ alloy having the largest $\mathrm{TM}$ contrast. 
Table 3-1 Designed waveguide contrast for TE and TM polarization for the three InGaAsP alloys.

\begin{tabular}{|l|l|l|}
\hline & $\Delta \mathbf{n}_{\mathrm{TE}}$ & $\Delta \mathrm{n}_{\mathrm{TM}}$ \\
\hline $\mathbf{Q}=1.2 \mu \mathrm{m}$ & $\mathbf{0 . 0 0 2 6}$ & 0.0040 \\
\hline $\mathbf{Q}=1.3 \mu \mathrm{m}$ & 0.0025 & 0.0043 \\
\hline $\mathbf{Q}=\mathbf{1 . 4} \mu \mathrm{m}$ & 0.0026 & 0.0047 \\
\hline
\end{tabular}

The control of the effective index step was achieved by varying the thickness of the undoped InP layer situated directly above the core from $230 \mathrm{~nm}$ for the $\mathrm{Q}=1.4 \mu \mathrm{m}$ to $360 \mathrm{~nm}$ for the $\mathrm{Q}=1.2 \mu \mathrm{m}$ material. The wafers were grown by molecular beam epitaxy (MBE) and the layer structure is shown in Figure 3-11.

Additionally, a graded doping profile was incorporated into the wafer layer design. The graded profile consisted of intrinsic layers next to the core, followed by a lower doping $\left(1 \times 10^{17}\right)$ cladding layer, and then a higher doping $\left(1 \times 10^{18}\right)$ cladding layer furthest from the core. The graded profile was incorporated to reduce potential barriers to current flow for the majority carriers while maintaining a relatively abrupt refractive index variation. Measured IV curves show a resistivity smaller than the CBE-grown wafers and comparable to the MOCVD-grown wafers with the missing undoped InP cladding layer.

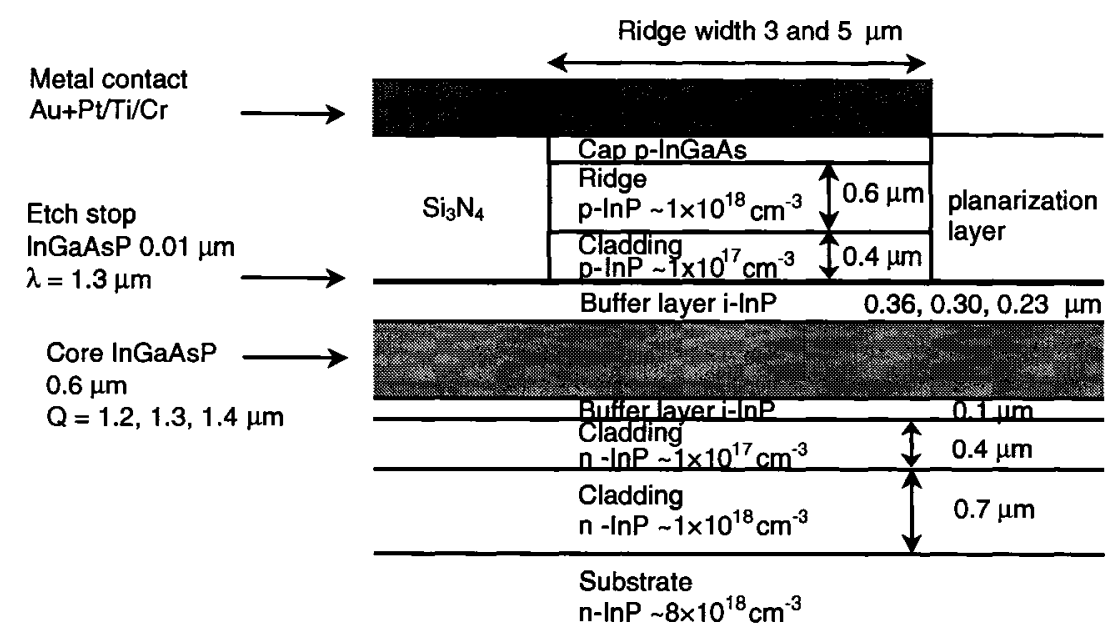

Figure 3-11 Schematic diagram illustrating the layer structure of the MBE grown InGaAsP/InP wafers. 
As mentioned previously, to compensate for the different refractive indices of InGaAsP quaternaries, the thickness of the undoped buffer layer above the core was made thicker $(0.36 \mu \mathrm{m})$ for the $\mathrm{Q}=1.2 \mu \mathrm{m}$ core alloy and made thinner $(0.23 \mu \mathrm{m})$ for the $\mathrm{Q}=1.4 \mu \mathrm{m}$ core alloy. This allows all three alloys to have the same effective index step between ridge and slab regions so that waveguide confinement remains consistent.

Finally, to increase switching speed, quantum well superlattice structures were designed for the core layer so that reverse biasing of the diode would induce electrooptical switching.

The wafer layer structures were identical to the MBE wafers described above except that the bulk InGaAsP core layer was replaced with QW superlattices. Two of the superlattice designs are simple barrier plus well structures (Figure 3-12 (a) and (b)). Larger electro-optical effects are observed for QW designs with bigger energy steps between barrier and well, with the exception of $\operatorname{InGaAs} / \operatorname{InP}$ structures for which As diffusion was thought to contaminate the InP barriers [68]. Thus we chose to grow Q1.1 barrier and Q1.5 well InGaAsP, similar to other QW optical switch designs [56]. A script (QuatAsP) written in the Quantum Physics Group of IMS calculates the bandgap of quantum well superlattice structures. It was used to design the width of the barrier and well layers to have a bandgap at $1420 \mathrm{~nm}$ at $300 \mathrm{~K}$.

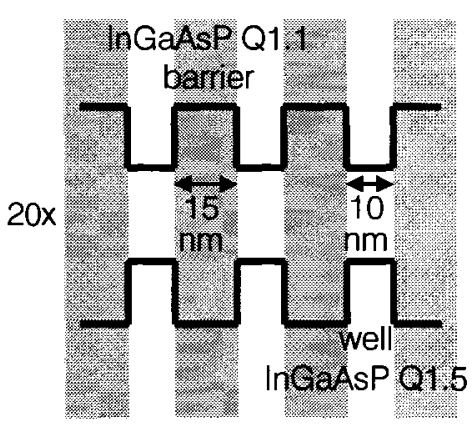

(a)

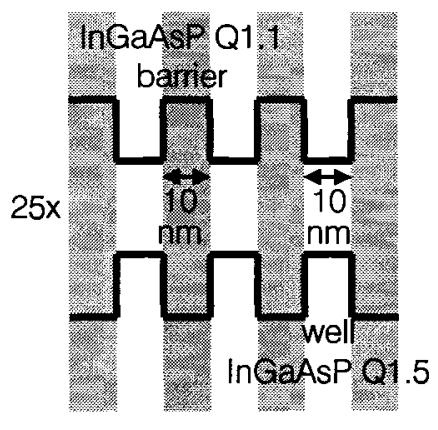

(b)

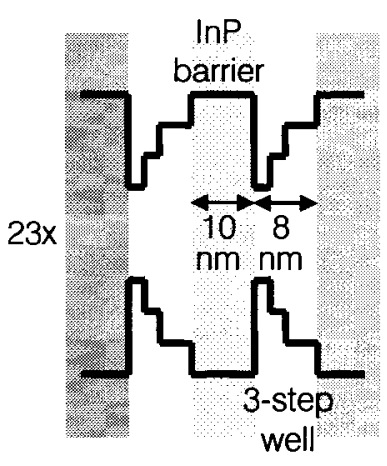

(c)

Figure 3-12 Energy band structure of the three QW superlattice structures (a) RQWa (b) RQWb, and (c) 3SQW.

The third QW wafer (Figure 3-12 (c)) uses a three-step quantum well (3SQW) design described by Mohseni et al. [65]. In this design, each well consists of a $2.1 \mathrm{~nm}$ layer of 
$\mathrm{In}_{0.54} \mathrm{Ga}_{0.46} \mathrm{As}$, a $2.1 \mathrm{~nm}$ layer of $\mathrm{Q}=1.55 \mu \mathrm{m}$ InGaAsP, and a $3.8 \mathrm{~nm}$ layer of $\mathrm{Q}=1.35$ $\mu \mathrm{m}$ InGaAsP. This 3SQW promises "higher electro-optical coefficient compared to conventional rectangular quantum wells (RQW) at very low optical loss values".

The waveguides were oriented along the secondary [011-] crystal axis in which direction the linear Pockels effect causes a positive index change. This allowed the linear effect to enhance the electro-optical effect, which is independent of crystal orientation. Further explanation is provided in the Appendix.

\subsection{Fabrication}

For this thesis, I designed a new mask that included many different variants of the RW-DOS device, in which all key design parameters were varied. These included DOS variations in branching angle $\left(0.78^{\circ}-2.03^{\circ}\right)$, waveguide width $(3 \mu \mathrm{m}, 4 \mu \mathrm{m}$, and $5 \mu \mathrm{m})$, electrode length $(264 \mu \mathrm{m}-484 \mu \mathrm{m})$, and electrode offset $(0.5 \mu \mathrm{m}, 0.75 \mu \mathrm{m}, 1 \mu \mathrm{m}, 1.5$ $\mu \mathrm{m}$, and $2 \mu \mathrm{m})$. Also included on the mask were straight waveguide and MZ interferometer test structures with the same variations in width, and for the MZ interferometers, variations in branching angle $\left(0.9^{\circ}\right.$ and $\left.1.05^{\circ}\right)$ and correspondingly electrode length $(2.8 \mathrm{~mm}$ and $3.2 \mathrm{~mm})$. The waveguide devices were designed using Optiwave 2D BPM_CAD 4.0 and exported in .dxf format to Autocad LT 2002 for layout. Labels, alignment marks, and dicing lines were inserted at this stage.

The mask was written by Adtek Photomask on a 4" plate of 0.090 " thick quartz glass. The devices were divided between two $12 \mathrm{~mm}$ square areas (labeled Mask A and Mask B) to be processed separately on quarters of 3 " InP wafers. Fabrication required two mask layers, one to define the waveguides and one to define the electrodes. The digitized data was chrome for the waveguide layer (light field) and clear for the electrode layer (dark field). The mask was written with right reading chrome down, address size of $25 \mathrm{~nm}$, and critical dimension of $0.5 \pm 0.1 \mu \mathrm{m}$. A supplementary mask included two additional layers, one for removing the edge bead (dark field) and another for ion-implantation (light field). 
Devices were patterned by contact photolithography and the waveguides were etched by a dry etch followed by a wet-etch to the etch stop [69]. The CBE samples were dry etched by chemically assisted ion beam etching (CAIBE), while the MOCVD and MBE samples were dry etched by inductively coupled plasma reactive ion etch (ICPRIE). Silicon nitride $\left(\mathrm{Si}_{3} \mathrm{~N}_{4}\right)$ was deposited for insulation and planarization, and metal stack of $500 \AA \mathrm{Ti} / 600 \AA \mathrm{Pt} / 5000 \AA \mathrm{Au}$ were deposited by metal sputtering and patterned by liftoff. The metal provided not only contact points, but also served as self-aligned shadow masks for the curved edges of the implantation region. Also, after samples were thinned and polished to $140 \mu \mathrm{m}$, the back of the substrate was coated with metal.

Since the two electrodes are separated by less than one micron at the Y-junction vertex, a method of electrical isolation was essential to create a high resistivity barrier between the contacts. Such isolation was achieved through ion implantation, using the electrodes as a mask, so that carrier injection would occur only directly beneath the electrode contact area. Good switch performance requires minimal current spreading, however the electrical isolation should not perturb the underlying guided optical fields.
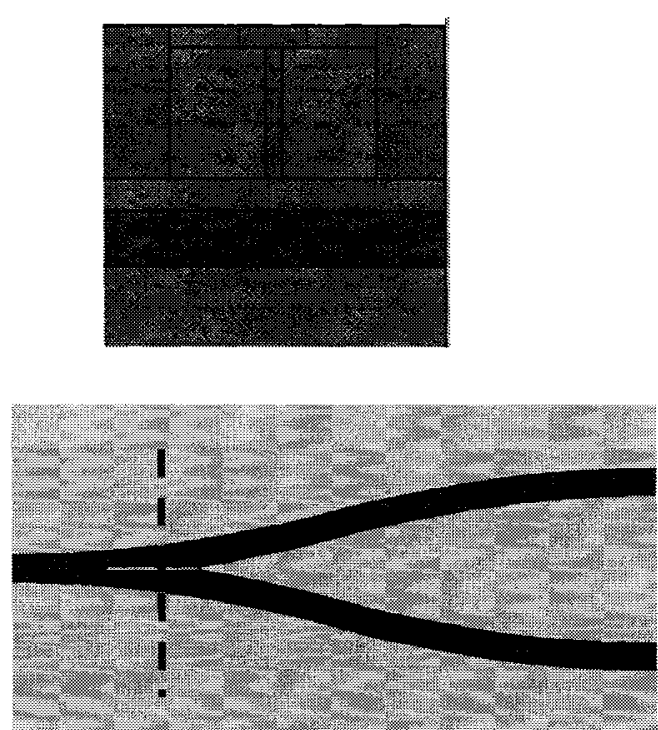

(a)
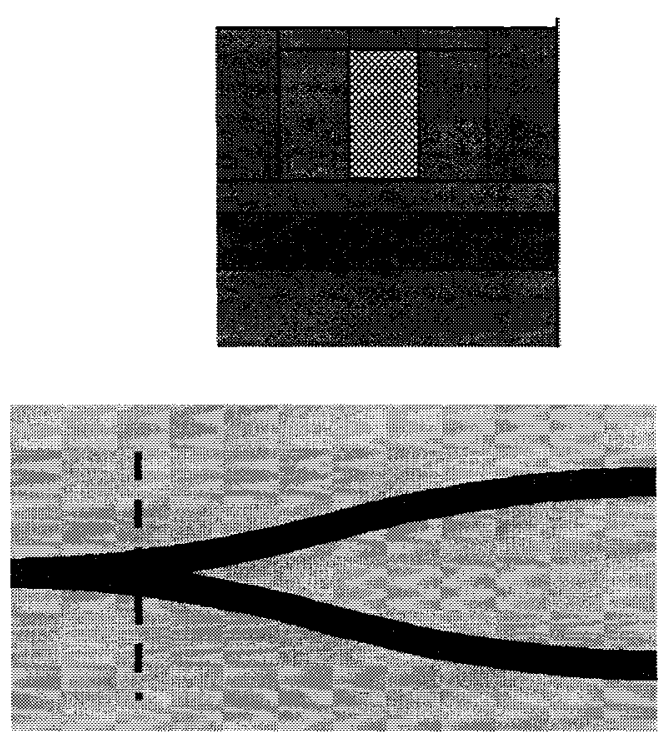

(b)

Figure 3-13 (bottom) Configuration of DOS with (a) physical isolation trench and (b) ion implant isolation and (top) cross-section of the DOS at the position indicated by the dashed line. 
Because ion implantation is costly and time consuming, an alternative method of electrode isolation was proposed and tested, i.e. a $0.5 \mu \mathrm{m}$ wide etched trench between the electrodes as shown in Figure 3-13 (a). A second set of DOS devices were thus fabricated on the MOCVD wafers using the isolation trench. The narrow trench width was smaller than the photolithographic patterning resolution so the devices were patterned by electron-beam (e-beam) lithography. The ridge waveguides and isolation trench were etched in the same step by inductively coupled plasma reactive ion etching (ICP-RIE) followed by a wet etch. The results of this study are presented in Section 5.2 .

\subsection{Device Modeling}

A two-dimensional beam propagation method (BPM) simulation, based on the waveguide structures of Figure 3-10 and Figure 3-11 and the effective index approximation, was used to calculate the optical output power in each arm of the switch as a function of negative index change. Figure 3-14 shows the normalized output for the biased and unbiased DOS arms as the refractive index is varied in the biased section. These simulations were carried out for the three different InGaAsP alloy wafers, using refractive index values calculated from equations in [58]. At the point of maximum switching contrast between the biased and unbiased arms, the output power of the unbiased arm is more than the sum of the output powers from both arms at zero index modulation. This is due to the low waveguide confinement, particularly of the $\mathrm{Q}=1.4$ $\mu \mathrm{m}$ MOCVD wafer, which allows a significant amount of input light to be lost at the Y-branch vertex when there is no applied bias. However, as the effective index of the biased arm is decreased and the DOS structure reduces to a single bent waveguide, this stray light is captured by the reconfigured single waveguide arm and appears at the unbiased output. Thus, the power output from the unbiased arm increases by both the power that had previously been guided by the biased arm as well as the power that had previously been lost at the vertex. Note that since the switch is always biased in operation, this junction loss is not a factor in the insertion loss. 
For the MOCVD wafers (Figure 3-14(a)), the InGaAsP alloy with the smallest bandgap, i.e. $0.88 \mathrm{eV}(\mathrm{Q}=1.4 \mu \mathrm{m})$, has the best calculated switching contrast at a small applied index modulation as compared to the InGaAsP alloys having larger bandgaps of $0.95 \mathrm{eV}(\mathrm{Q}=1.3 \mu \mathrm{m})$ and $1.03 \mathrm{eV}(\mathrm{Q}=1.2 \mu \mathrm{m})$. This is due to the lower ridge-slab index contrast of structures on the $\mathrm{Q}=1.4 \mu \mathrm{m}$ bandgap wafer. This smaller contrast requires a smaller index modulation to reduce the waveguide effective index enough to form the outer boundary of the unbiased waveguide arm. This difference is eliminated for the MBE grown wafers and reflected in the plot of Figure 3-14(b) which shows the same switching behaviour for the simulated devices of all three quarternaries.

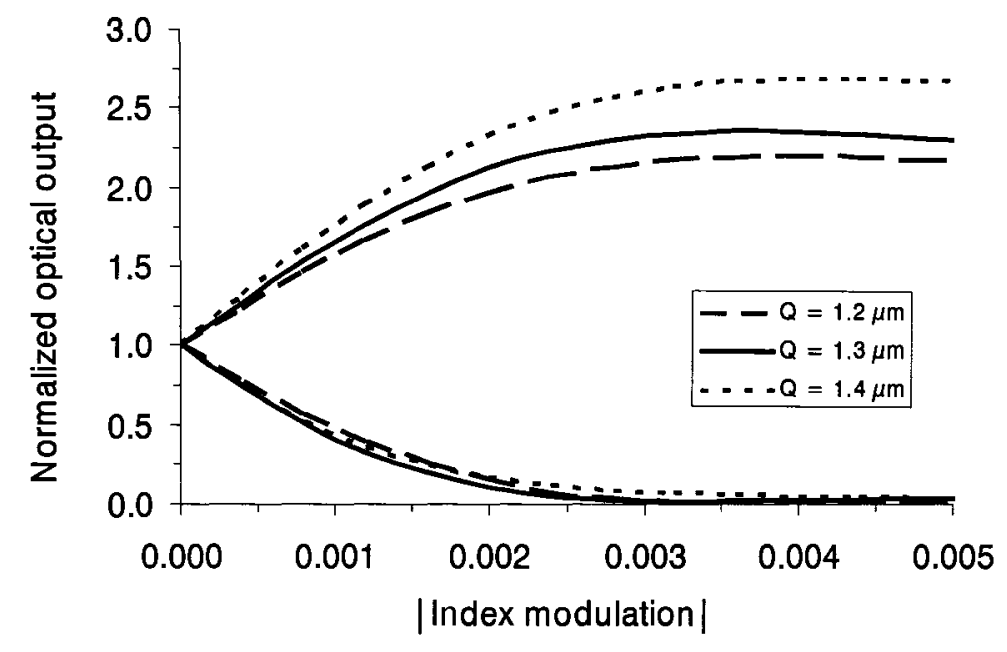

(a)

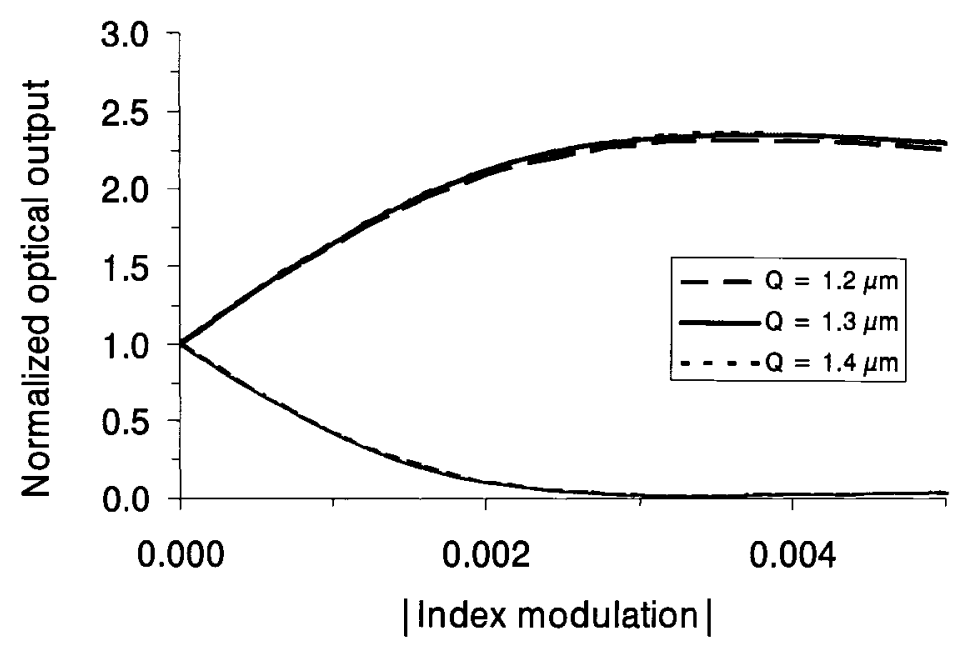

Figure 3-14 BPM simulation results showing normalized optical output power of TE polarization as a function of applied negative index change in the biased (bottom) and unbiased (top) arms, comparing layer designs for (a) MOCVD and 
(b) MBE wafers with InGaAsP cores of bandgaps $\mathrm{Q}=1.2 \mu \mathrm{m}, 1.3 \mu \mathrm{m}$, and 1.4 $\mu \mathrm{m}$.

The calculated contrast ratio between the two arms for the $\mathrm{Q}=1.3 \mu \mathrm{m}$ InGaAsP quaternary is shown in Figure 3-15. The contrast is highest when the electrically induced $\Delta \mathrm{n}$ is equal to the effective index difference, $\delta \mathrm{n}_{\text {eff }}$, between the ridge waveguide and the adjacent slab region. This occurs at $\Delta \mathrm{n}=-0.0025$ for TE polarized light, and at $\Delta \mathrm{n}=-0.0043$ for TM polarized light. This difference arises from the intrinsic polarization dependence of the ridge and slab waveguide effective index values used in our idealized effective index model. For either polarization, the contrast ratio decreases for $\Delta \mathrm{n}$ larger than $\delta \mathrm{n}_{\text {eff }}$ due to the mode mismatch between the carrierinduced waveguide near the $\mathrm{Y}$-junction and the passive output waveguide section. However, the contrast ratio remains better than $20 \mathrm{~dB}$ for both TE and TM light, for an induced $\Delta \mathrm{n}$ greater than about -0.003 .

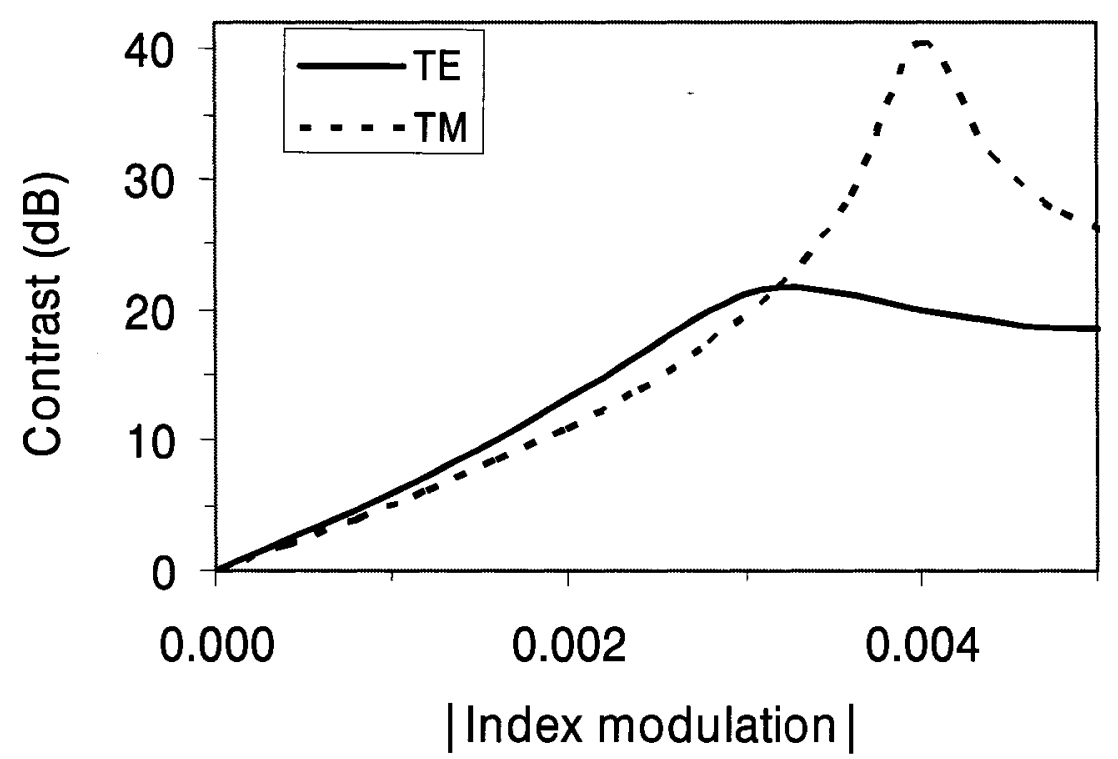

Figure 3-15. BPM simulation results showing calculated switch contrast ratio of power between the two DOS arms as a function of applied negative index change comparing $\mathrm{TE}$ and $\mathrm{TM}$ polarizations for $\mathrm{Q}=1.3 \mu \mathrm{m}$.

The optical model described above assumes a sharp refractive index step at the electrode edge and hence neglects current spreading and carrier diffusion, which may broaden and smooth the carrier distribution and resulting index profile well beyond the electrode edges. Our studies, detailed later in Section 5.2, compare electrical 
isolation of the two electrodes using ion implantation and an etched trench. It demonstrates that current spreading is an important factor limiting the performance of the RW-DOS. Switches using implant isolation had better performance not because of the electrical isolation, which was comparable for both methods, but because implant isolation is better able to suppress current spreading at the electrode edges as the carriers propagate through the upper cladding layers of the pin diode structure [70]. These results underline the benefit of using the electrodes as a shadow mask for the ion implant.

Two-dimensional drift-diffusion carrier dynamics simulations were performed using values for electron and hole mobility from [71] to determine the extent of carrier diffusion and current spreading under the biased electrode. The simulated band structure according to the layer structure of Figure 3-9 is illustrated in Figure 3-16. The calculated distribution of holes in the core layer drops to half of its maximum value at a position about $1 \mu \mathrm{m}$ beyond the edge of the electrode. To compensate for the broadened carrier distribution in the waveguide core, the electrodes on the fabricated devices were displaced outward by $1 \mu \mathrm{m}$ from their nominal position in our optical model, as indicated in Figure 3-2 (a). The index gradient resulting from carrier diffusion was not included in the BPM modeling results presented here. Although possible in principle, simulating these effects accurately requires full three-dimensional calculations of both carrier dynamics and optical propagation, an undertaking well beyond the scope of this work.

\subsection{Summary}

The RW-DOS allows for smaller electrodes and device footprint than many existing waveguide-based switch designs. Actuation by carrier injection provides wavelength independent and broadband operation at nanosecond speeds. Optical and electrical simulations were performed prior to the design of the RW-DOS to define device parameters such as electrode offset and core InGaAsP alloy. Subsequently, improvements were made to the layer structure to optimize performance, such as reducing 
current by grading doping profiles and increasing switching speed by employing electro-optical effects in quantum well cores.

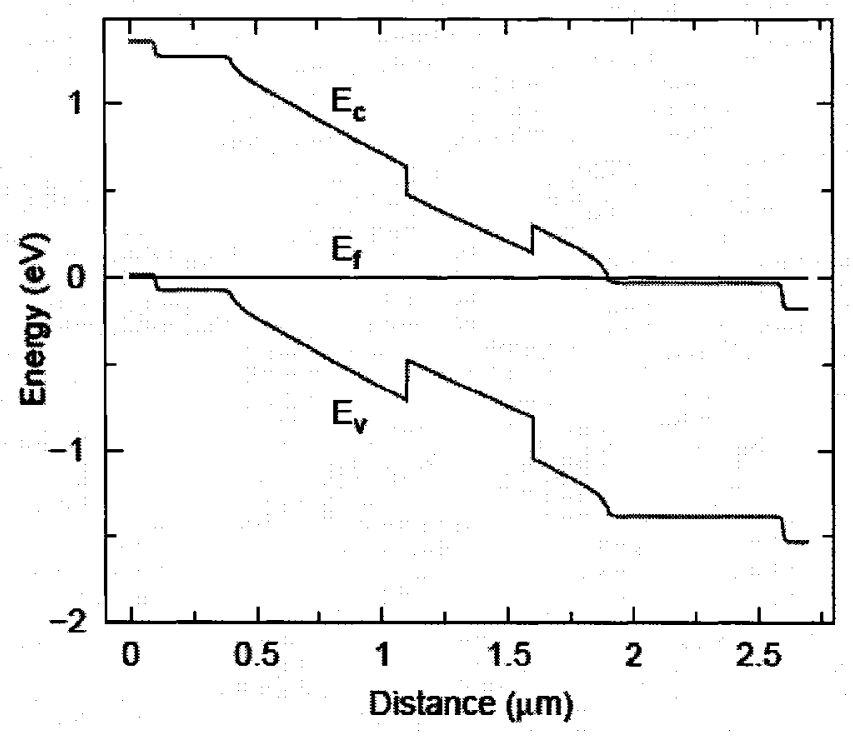

(a)

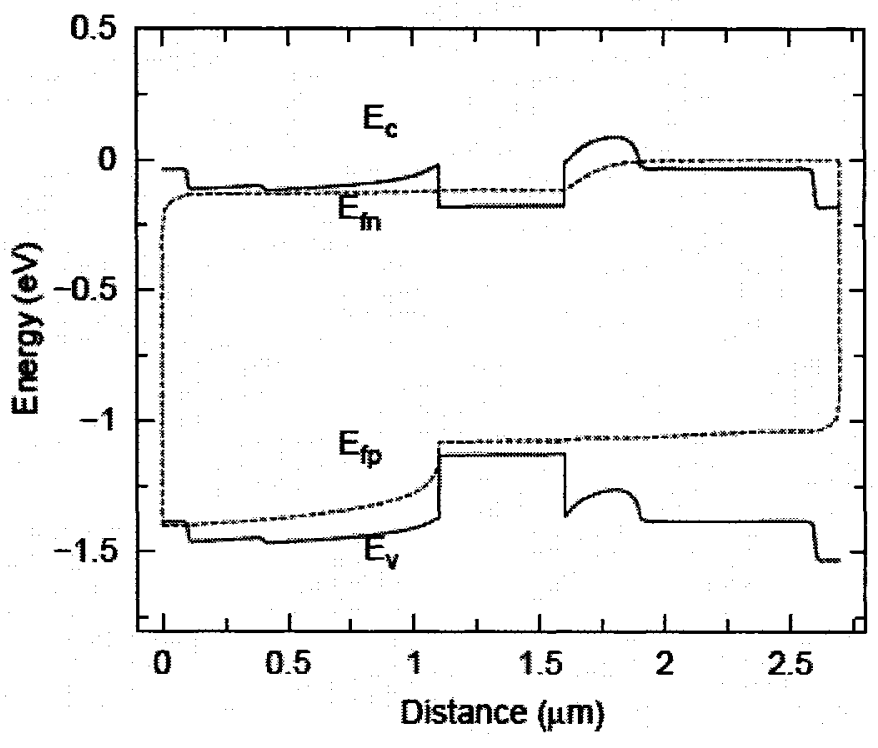

Figure 3-16 Band diagram through the center of the waveguide ridge from the top p-doped layer down, at (s) zero applied bias and (b) with $1.4 \mathrm{~V}$ forward bias. 


\section{Chapter 4}

\section{STUDY OF CARRIER INJECTION}

\subsection{Characterization Tools and Techniques}

\subsubsection{Experimental setup}

All device characterization was performed at IMS facilities using the experimental setup shown in Figure 4-1. For C-band broadband measurements, an erbium amplified spontaneous emission (ASE) unit (Agilent 83438A) amplified by an Oprel erbium doped fiber amplifier (EDFA) serves as the optical source. The maximum output power of the two in series is $\sim 10 \mathrm{~mW}$ at $-1550 \mathrm{~nm}$. For measurements at individual wavelengths or in the L-band, two tunable lasers were employed: Agilent 81640A 1600 $\mathrm{nm}$ and $81682 \mathrm{~A} 1550 \mathrm{~nm}$.

The output from each source is connected through an FC/APC connectorized polarization-maintaining fiber to a free space adjustable half wave plate and polarizer. It is then coupled to another polarization maintaining fiber with a taper at the output end. This lensed fiber is placed in a fiber chuck, which is mounted onto a Melles Griot xyz translation stage for alignment of the fiber to the device under test (DUT). The DUT is mounted on a Melles Griot xz translation stage with pitch and yaw positioning. A microscope (Nikon SMZ800) is placed above the DUT to view the top of the sample. At the output side of the DUT, another lensed fiber mounted to another translation stage collects the output light. For slow speed measurements, this light is sent to a 
photodetector (Thorlabs PDA 255), which is connected to a $100 \mathrm{MHz}$ oscilloscope (Tektronix TDS 3012). To record the time response of short pulses, a sampling oscilloscope (Agilent Infinium 86100A) with a detector (86109A) capable of $30 \mathrm{GHz}$ frequency is employed.

A pulse generator (HP 8116A) provides electrical signals through a probe (Picoprobe 7-60 and 7-22) to the electrodes on the samples. The probe is mounted on an XYZ translation stage (Quater Research and Development XYZ 500 MLS) and is grounded to the bottom of the samples by an alligator clip attached to the aluminum sample mount. The pulse generator provides rectangular pulses with minimum rise and fall times of 3 to 4 nanoseconds. The response time of the system is about 7 ns as determined by a measurement of a rectangular electrical pulse transmitted through an electrode contacted by two probes. Two pulse generators are connected in series when triangular pulses are required. One generator supplies rectangular pulses and serves as the trigger to the second generator, which outputs triangular pulses at the frequency of the trigger. A faster pulse generator (Philips PM 5770) capable of up to $100 \mathrm{MHz}$ and rise/fall times of $<4 \mathrm{~ns}$ stopped functioning, and so some results are limited by the experimental setup to several nanoseconds while others are limited to $7 \mathrm{~ns}$. The current drawn from the pulse generator is measured by passing the bias lead through an inductive current loop (American Laser Systems $711 \mathrm{~S}$ ) before the DUT. The current probe has a bandwidth of $8 \mathrm{kHz}$ to $100 \mathrm{MHz}$ with a minimum rise time of 3.5 ns.

During initial alignment of a DUT, the output scheme described above is replaced with the alignment schemes illustrated in the dashed grey box of Figure 4-1. First, the lensed fiber at the output is replaced with a $10 \times$ microscope objective with 0.25 numerical aperture (NA), which focuses the output light into an infrared (IR) camera (Electrophysics micronviewer 7290A). The images of the input and output facets are displayed on a television monitor to assist this initial coupling into the waveguide. Then the lensed fiber is replaced and the output sent to a power meter (Newport $11830-\mathrm{C})$, where the alignment is adjusted to maximize measured output power. 


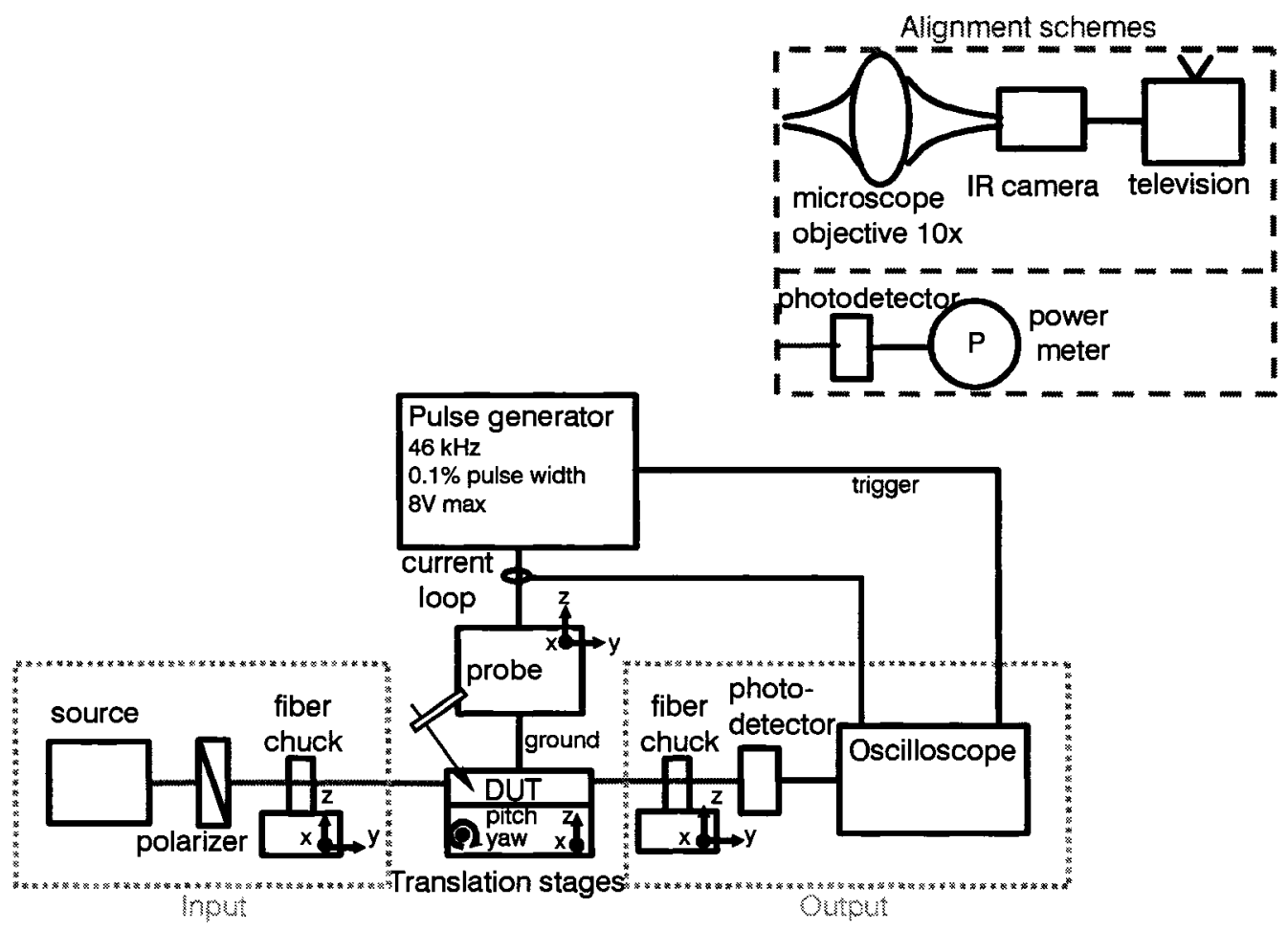

Figure 4-1 Experimental setup for device characterization illustrating the optical source and detection systems, and the electrical signal system.

\subsubsection{Mach-Zehnder interferometer index measurements}

Several test structures were included in the masks to allow material characterization and a study of carrier injection. One of these was the Mach-Zehnder interferometer, which is a device in which input light is split into two paths and then recombined. When the two path lengths are the same, the recombined signals constructively interfere. A difference of half a wavelength, or $\pi$ in phase, in the optical path length $\Delta\left(n_{\text {mode }} \times L\right)$ between the two paths result in destructive interference of the recombined signals. Thus by observation of the interferometer output power, the change in optical path length can be determined. MZ interferometers are often used to determine changes in path length $L$ and waveguide effective refractive index $n_{\text {mode }}$, the two components of optical path length. 
The output power of a MZ interferometer is a sinusoidal function of the phase difference $\Delta \phi$ between its two arms. This phase difference is related to the change in modal refractive index $\Delta n_{\text {mode }}$ by the following equation:

$$
\Delta \phi_{M Z}=2 \pi \frac{\Delta \mathrm{n}_{\text {mode }} L}{\lambda}
$$

where $\lambda$ is the wavelength of the light. Thus the output power is periodic with index modulation, with period $\Delta n_{\text {mode }}=\lambda / L$ (see Figure 4-2, mode index). That is, the index modulation may be correlated to the MZ output power periodicity.

In the proposed DOS, carrier injection directly alters the core material refractive index, which determines the modal index. However, the relationship between the two is not linear, and so the variation of output power with applied current does not have a fixed period as shown in Figure 4-2. This nonlinear relationship must be determined through a mode solver, as described in Section 3.1.1.

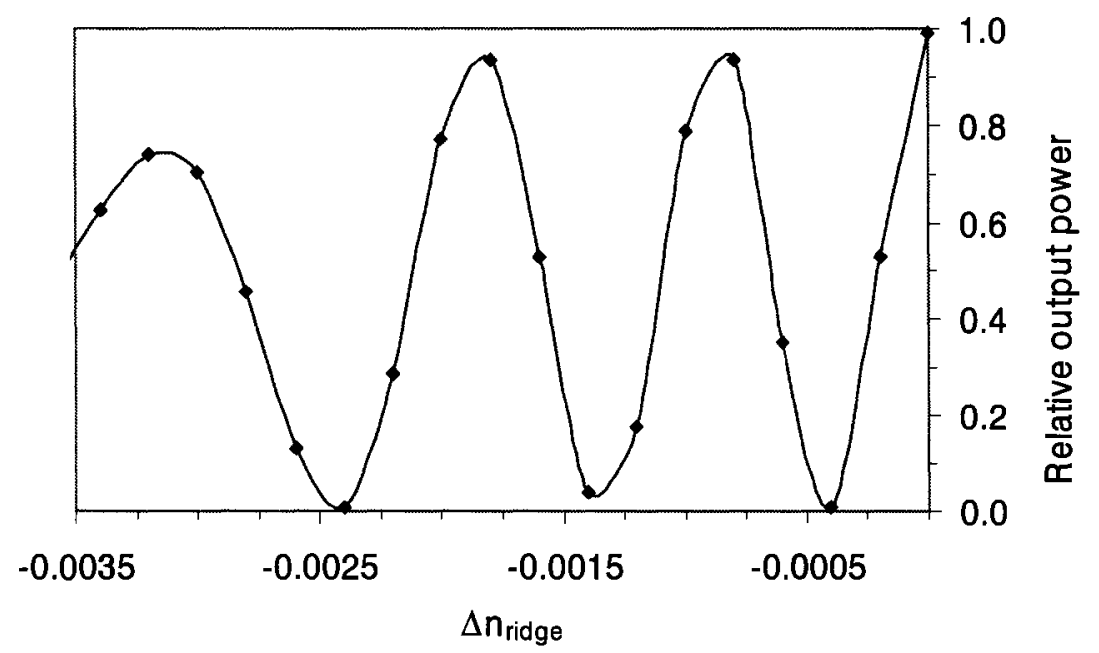

Figure 4-2 Plot of BPM generated fringes of $\mathrm{MZ}$ interferometer versus ridge effective refractive index $\Delta \mathrm{n}_{\text {ridge }}$.

Although the fringe periods are wavelength dependent, a broadband source with a wavelength spread of $30 \mathrm{~nm}$ produces nearly the same fringe spacing as a single wavelength source at the center of the broadband range. Compared with a single wavelength laser source, a broadband source has the benefits of: 
1. washing out Fabry Perot fringes from facet reflections, and

2. providing higher power.

Matlab simulation results ${ }^{6}$, presented in Figure 4-3, compare the broadband (solid line) and single wavelength (dotted line) interference fringes for a $3.2 \mathrm{~mm}$ long electrode. The results, show that the fringe spacing is nearly the same for both sources over an index range of 0.02 , although the fringe amplitude is modulated by a beat pattern due to the presence of multiple wavelengths. However, since only measurements of the fringe periods are required to measure index change, the broadband source will provide the same values of index change as a single wavelength source at $1550 \mathrm{~nm}$.

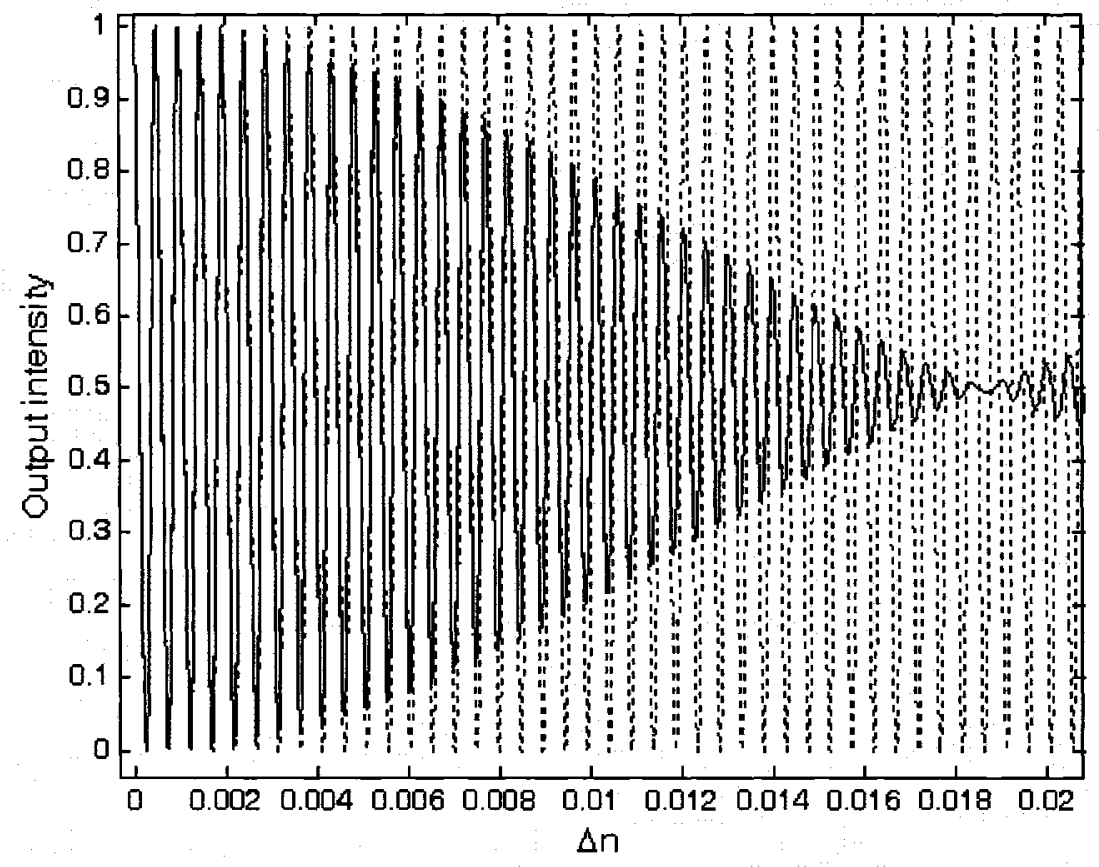

Figure 4-3 Plot of Matlab simulation results comparing MZ fringes using a broadband (solid line) and a single wavelength (dotted line) source.

From the MZ interferometer fringe data, the index change $n$ is determined as a function of applied current density $j$. This is done by relating the experimentally

${ }^{6}$ According to the ideal $\mathrm{MZ}$ output formula, intensity $=1 / 4^{*}\left(2+2^{*} \cos (2 \pi \Delta \mathrm{nL} / \lambda)\right)$. For the broadband source, the total intensity is integrated over a $1525-1565 \mathrm{~nm}$ range in wavelength. 
measured current density to the calculated refractive index change of each fringe period. The index change was calculated using a mode solver to relate the material refractive index change in the waveguide core to the ridge effective index change simulated in the BPM program. A slope $d n / d j$ is then extracted from a first order fit of the $n$ versus $j$ data.

\subsubsection{Fabry Perot interferometer index measurements}

Another test structure is the Fabry Perot interferometer, in which multiple reflections of light from the two end facets of a straight waveguide produce interference fringes with intensity:

$$
I=I_{0} \frac{(1-R)^{2} e^{-\alpha L_{w}}}{1+R^{2}-2 R \cos \left((2 \pi / \lambda)\left(2 n L_{w}+2 \Delta n L\right)\right)}
$$

where $I_{0}$ is the input intensity, $R$ is the reflection coefficient of each end facet, $\lambda$ is the signal wavelength, $\alpha$ is the loss coefficient, $L_{w}$ is the waveguide length, and $L$ is the electrode length.

The depths of the fringes are much smaller than those of the MZ interferometer and are dependent upon the reflectivity $R$ of the facets as well as the waveguide loss $e^{-a L_{w}}$. Thus the loss can be calculated from the maximum $I_{\max }$ and minimum $I_{\min }$ intensities of the fringes [72]:

$$
e^{-\alpha L}=\frac{1-\sqrt{1-\gamma^{2}}}{\gamma R}
$$

where

$$
\gamma=\frac{I_{\max }-I_{\min }}{I_{\max }+I_{\min }}
$$

The phase difference, given the same electrode length, is double that of a MZ interferometer because reflected light must travel twice the length of the waveguide to return to the output facet. Thus, the FP interferometers provide greater resolution. By observing the period of the FP interferometer fringes, the change in optical path 
length under the electrode can be determined in the same manner as for a $\mathrm{MZ}$ interferometer, with the phase difference related to the change in refractive index $\Delta n_{m}$ by:

$$
\Delta \phi=2 \pi \frac{\Delta \mathrm{n}_{\mathrm{mode}} 2 L}{\lambda}
$$

\subsection{Modeling}

\subsubsection{Carrier Lifetime}

Upon the application of a forward biased potential gradient across the DOS pin diode, carriers are injected into the waveguide core at a rate proportional to the applied current density, and are removed at a rate dominated by carrier recombination. The carrier concentration $N$ can therefore be related to applied current density $j$ and carrier lifetime $\tau$ by the following equation:

$$
j=\frac{q N d}{\tau}
$$

where $q$ is the electron charge and $d$ is the thickness of the core layer. This assumes that all carriers are injected into the core layer beneath the ridge, remaining there until they recombine, and therefore does not include the effects of current spreading or diffusion of carriers in the core. The total carrier recombination rate is determined by the combined effect of radiative (optical) and nonradiative (Shockley-Reed-Hall (SRH) and Auger) recombination:

$$
\frac{1}{\tau}=\frac{1}{\tau_{\text {aug }}}+\frac{1}{\tau_{S R H}}+\frac{1}{\tau_{\text {rad }}}
$$

In this simple picture, the intrinsic time response of a carrier injection based modulator or switch will be determined by the carrier lifetime. The solution to a simple first order rate equation $\mathrm{dN} / \mathrm{dt}=-\mathrm{N} / \tau$, predicts that based on carrier injection and 
recombination rates both the rise and decay time of the carrier density, in response to a square current pulse, are equal to the carrier lifetime $\mathrm{N}=\mathrm{N}_{0} \mathrm{e}^{-\mathrm{t} / \tau}$.

The effects of each of the above recombination mechanisms on carrier lifetime are shown in Figure 4-4. For the figure, a SRH lifetime of $7 \mathrm{~ns}$ is used, based on experimental values obtained as described later in Section 5.3. The Auger coefficient is $3.33 \times 10^{-29} \mathrm{~cm}^{6} / \mathrm{s}$, and the radiative coefficient is $1.25 \times 10^{-10} \mathrm{~cm}^{3} / \mathrm{s}$, interpolated from Agrawal and Dutta [73]. According to Figure 4-4, in the operating region of the device (carrier concentration $<4 \times 10^{17} \mathrm{~cm}^{-3}$ ), the SRH recombination largely determines carrier lifetime. Optical recombination is only significant at sufficiently high carrier concentrations or with sufficiently pure device material such that radiative and SRH recombination times are comparable. From the observed response times of 5 to $40 \mathrm{~ns}$ of our various devices, we conclude that our material is limited by $\mathrm{SRH}$ recombination in the region of operation below $4 \times 10^{17} \mathrm{~cm}^{-3}$. Thus the response time of the RW-DOS devices depends on the density of recombination centers in the InGaAsP alloy due to impurities and crystalline defects.

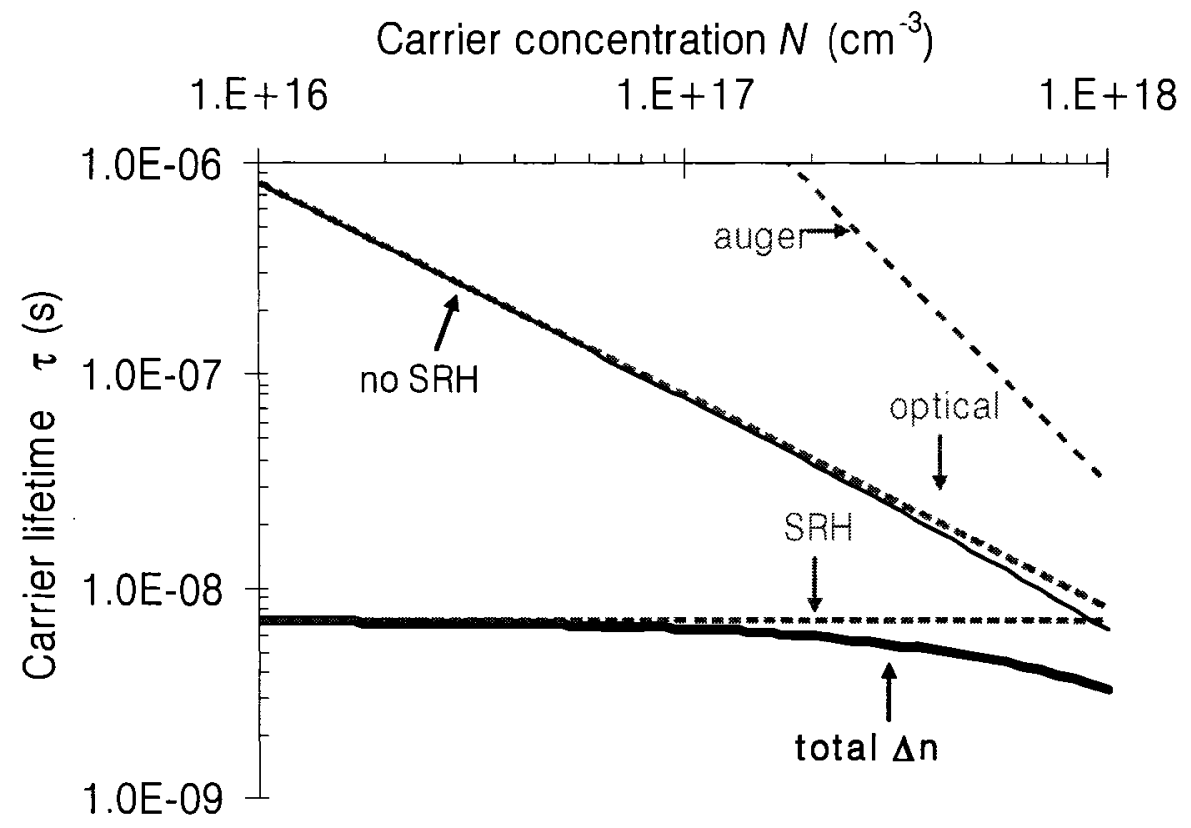

Figure 4-4 Carrier lifetimes as a function of carrier concentration modeled with Auger, Shockley-Reed-Hall (SRH), and optical recombination. The total lifetime is shown by the thick solid black line, whereas the lifetime with no SRH mechanism (i.e. only Auger and optical recombination) is shown by the thin solid black line. 


\subsubsection{Material Bandgap}

The presence of free carriers in a semiconductor modifies its absorption spectrum, and through the Kramers-Krönig relations the corresponding refractive index change can be calculated as described previously in Section 3.2.1. The wavelength dependence of the absorption spectrum translates to a dependence of refractive index modification on material bandgap. Thus at the fixed signal wavelength, the magnitude of index change induced by an applied current density is larger for an InGaAsP quaternary with a smaller bandgap. This comes at the expense however of increased material absorption.

Free carriers cause an index change through a combination of free carrier absorption, bandfilling, and band-gap shrinkage (BGS) [58-60]. This total index change is calculated for three InGaAsP alloys $(\mathrm{Q}=1.4 \mu \mathrm{m}, 1.3 \mu \mathrm{m}$, and $1.2 \mu \mathrm{m})$ at a wavelength of $1.55 \mu \mathrm{m}$ and is plotted in Figure 4-5(a). The model for free carrier absorption and values of carrier mobility and effective mass, are taken from Weber [58]. The model for bandfilling is taken from Bennett et al. [59], and the model for bandgap shrinkage is taken from Botteldooren and Baets [60].

The models of BGS in [59] and [58] assume approximations that predict a region of positive index change between carrier concentrations of about $5 \times 10^{17}$ and $2 \times 10^{18} \mathrm{~cm}^{-3}$, as shown in Figure 4-5(a). However, using Mach-Zehnder (MZ) or Fabry-Perot (FP) interferometer measurements, neither we nor previous studies [74, 75] have ever experimentally observed this positive index change region. In contrast, the BGS equation according to Botteldooren and Baets [60] takes temperature dependence into account and predicts a much smaller BGS shift at room temperature. The corresponding carrier induced index changes including this model for BGS are presented in Figure 4-5(b) and show no region of positive index change. 


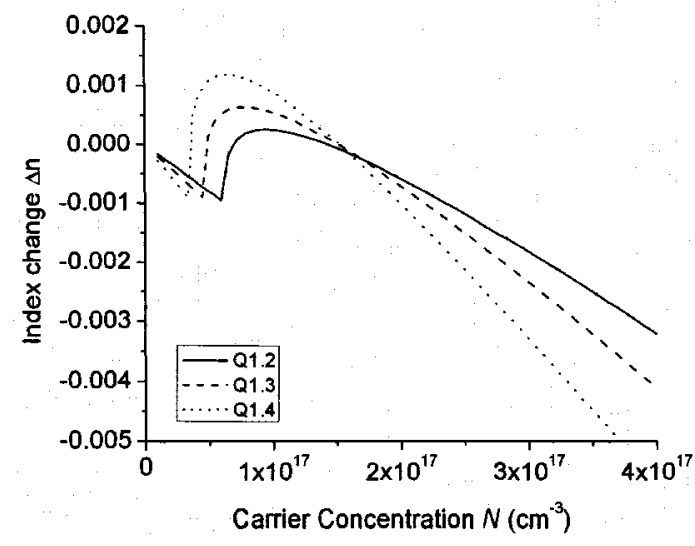

(a)

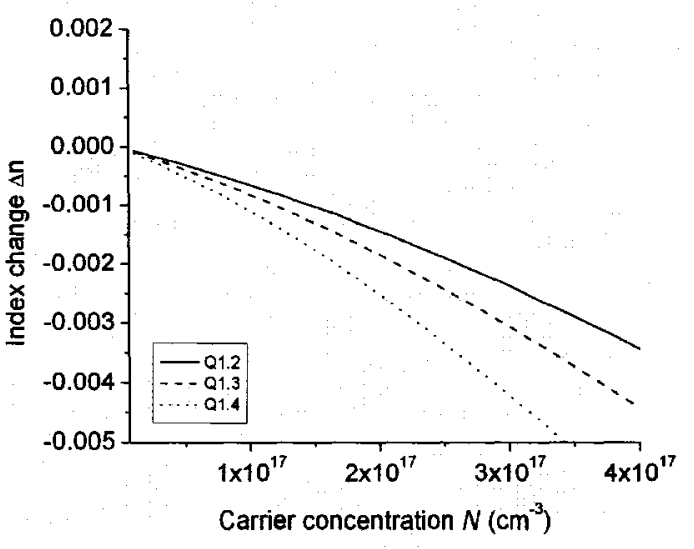

(b)

Figure 4-5 Modeled values of index change at $1550 \mathrm{~nm}$ versus carrier concentration $N$ for InGaAsP of bandgap (Q) $1.4 \mu \mathrm{m}, 1.3 \mu \mathrm{m}$, and $1.2 \mu \mathrm{m}$ using models of bandgap shrinkage according to (a) Weber [58] and (b) Botteldooren and Baets [60].

Figure 4-5(b) shows that alloys with a smaller bandgap, closer to the interrogating wavelength, produce larger index changes for a given carrier concentration than alloys with a larger bandgap. This is because carrier induced shifts in the absorption spectrum modify material absorption, and consequently refractive index, predominantly near the band edge. Thus use of a smaller bandgap alloy should reduce current, and therefore power requirements, of a switch device employing carrier induced index modulation. However this may result in higher material absorption through the Urbach tail [76], an exponential rise in absorption due to localized states at the bandedge from structural (e.g. doping) and thermal disorder. The higher material absorption results in higher device insertion loss, which is in fact experimentally observed when comparing the device insertion loss and spectral transmission of the different quaternary InGaAsP alloys.

\subsubsection{Carrier Diffusion}

The RW-DOS operates through a reconfiguration of branch waveguides using a localized negative refractive index gradient. Thus the shape of the index modulation is very important to the operation of this switch design, as it defines the reconfigured 
index profile. Establishing an accurate index modulation shape is dependent upon several factors, including the extent of carrier diffusion.

Electrical performance was simulated with 2D drift-diffusion calculations using mobilities from Sotoodeh et al. [71]. Figure 4-6 shows the simulation results of the hole concentration in the core layer for SRH recombination times covering the range of experimentally observed response times, namely 4,15 , and 40 ns. The peak hole concentration was chosen to correspond to the index change required for optimal switching, $\delta \mathrm{n}_{\text {eff }}$, as described earlier. The positions of the biased and unbiased electrodes are indicated at the top of the graph. The calculated hole distributions show that a smaller current density $\left(0.51 \mathrm{kA} / \mathrm{cm}^{2}\right.$ versus $\left.1.53 \mathrm{kA} / \mathrm{cm}^{2}\right)$ will produce a comparable carrier density when the recombination time is larger (40 ns versus $4 \mathrm{~ns}$ ). However, for longer lifetimes, the hole distribution is also broader because the carriers have more time to diffuse sideways away from the biased electrode thereby washing out the sharp index step required for switching. Therefore, while a longer carrier lifetime will result in higher carrier density for a given current, the increase in lateral carrier diffusion can cause the achievable switching contrast ratio to deteriorate.

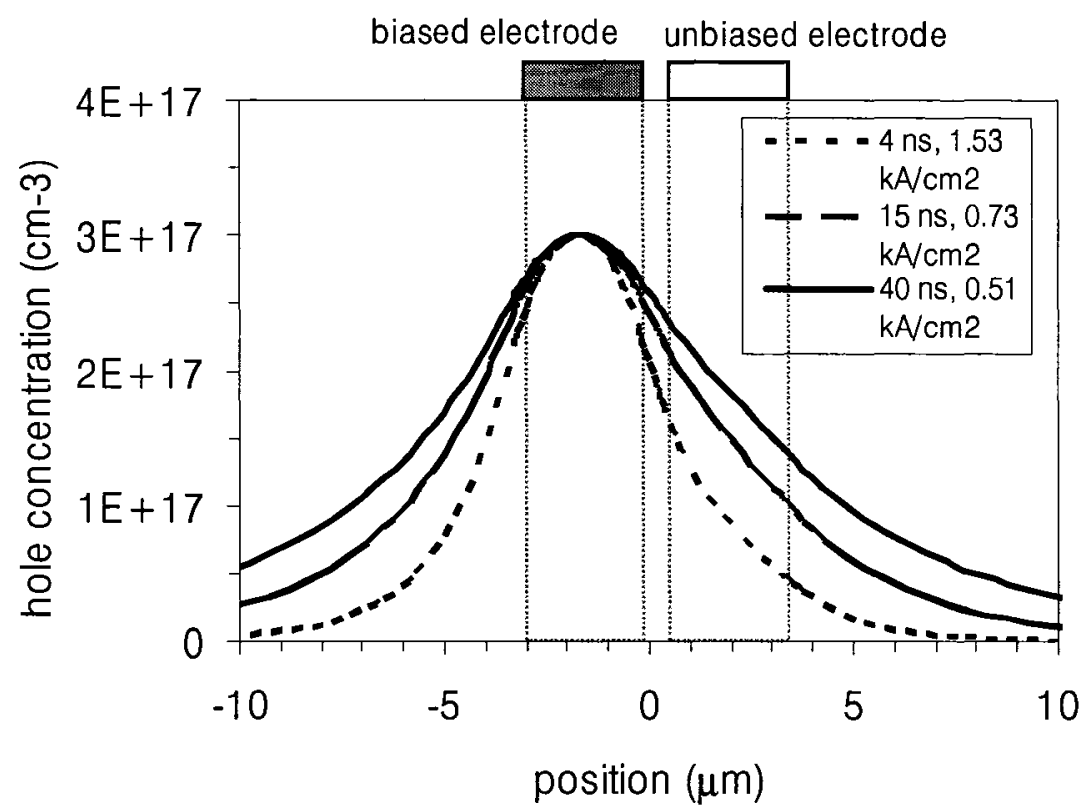

Figure 4-6 Modeled hole concentration along the cross section through a $\mathrm{Q}=1.3 \mu \mathrm{m}$ InGaAsP core layer as a function of lateral position. 


\subsection{Experimental Results}

MZ interferometer devices were fabricated from the three InGaAsP quaternaries using the same wafers as described in Section 3.3. The interferometers consist of a Yjunction branching into two $3.2 \mathrm{~mm}$ long straight waveguides, which then combine through a second Y-junction into one output waveguide, as shown in Figure 4-7. The branching angles of both Y-junctions are $1.05^{\circ}$, an electrode on one arm is $3.2 \mathrm{~mm}$ long, and the ridge waveguides are $3 \mu \mathrm{m}$ wide. The layer structure and fabrication are identical to the previously described RW-DOS devices.

Figure 4-7 Illustration of Mach-Zehnder interferometer device.

Light from a broadband ( $\lambda=1530-1560 \mathrm{~nm}$ ) erbium doped fibre source was coupled into the input waveguide of a $\mathrm{MZ}$ interferometer through a tapered fibre. The broadband source produces the same fringe pattern as a single wavelength source at $1550 \mathrm{~nm}$, but washes out FP interference fringes and provides larger optical power as discussed in the previous section. The optical power in the output arm was collected using a tapered fibre coupled to a fast InGaAs photodiode. By applying a triangularshaped current pulse to one arm of the MZ devices, the output power is swept through a series of interference fringes, as shown in Figure 4-8. The negative excursion after the triangular current pulse is an artifact of the current probe. The rapidly oscillating short period fringes follow the applied bias exactly, to within the $7 \mathrm{~ns}$ time resolution of our setup. On the other hand, the long tail, which decays away slowly following the current pulse, is caused by a thermo-optically induced index change due to heating of the biased waveguide. This tail corresponds to only a small fraction of one fringe. It demonstrates the smaller size of the thermally induced index change relative to the carrier induced index modulation, as well as the longer thermal recovery time. 


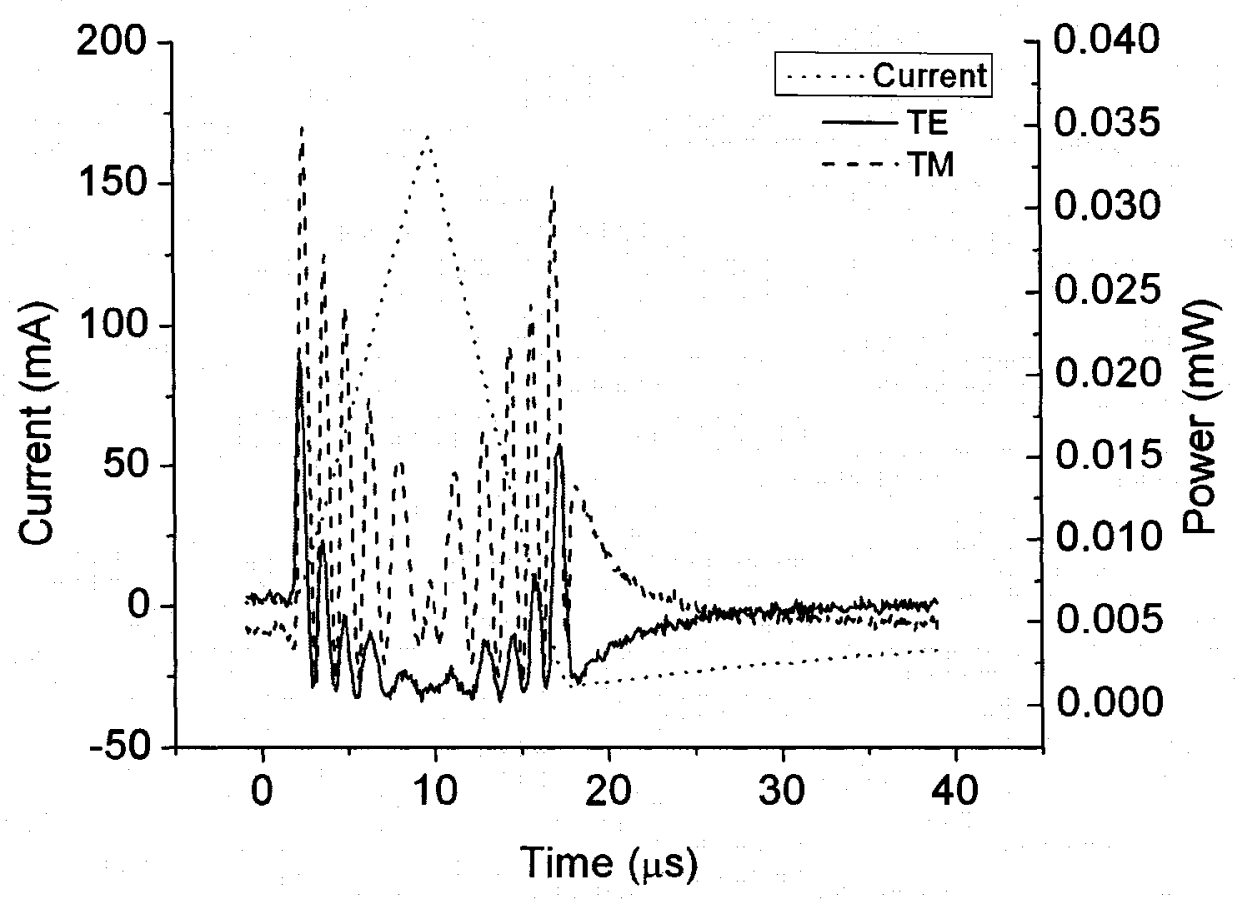

Figure 4-8 Measured optical output power from a MZ waveguide interferometer driven by a triangular current pulse, for a wafer grown by MOCVD with a core layer alloy of bandgap Q1.4 $\mu \mathrm{m}$.

From this MZ interferometer fringe data, the index change $n$ in the core layer induced by the applied current density $j$ could be determined. Since the electrode length is known, the measured current density is easily related to the effective refractive index change corresponding to each fringe oscillation. The corresponding index change in the waveguide core is then determined using the two-dimensional effective index model described previously. As mentioned, the optical model neglects lateral carrier diffusion, and assumes that the material index change occurs only beneath the waveguide ridge and is spatially uniform within this region.

The wavelength dependence of index modifications was determined by interrogating the MZ interferometers with tunable lasers operating in the $C(1450$ to $1590 \mathrm{~nm})$ and L-band (1500 to $1650 \mathrm{~nm}$ ). At each wavelength, the change in core material index with applied current density, $d n / d j$, was determined from the experimental data using the effective index model as described. The experimental results are shown in Figure 4-9 for waveguides with $\mathrm{Q}=1.2,1.3$ and $1.4 \mu \mathrm{m}$ InGaAsP alloy cores. 


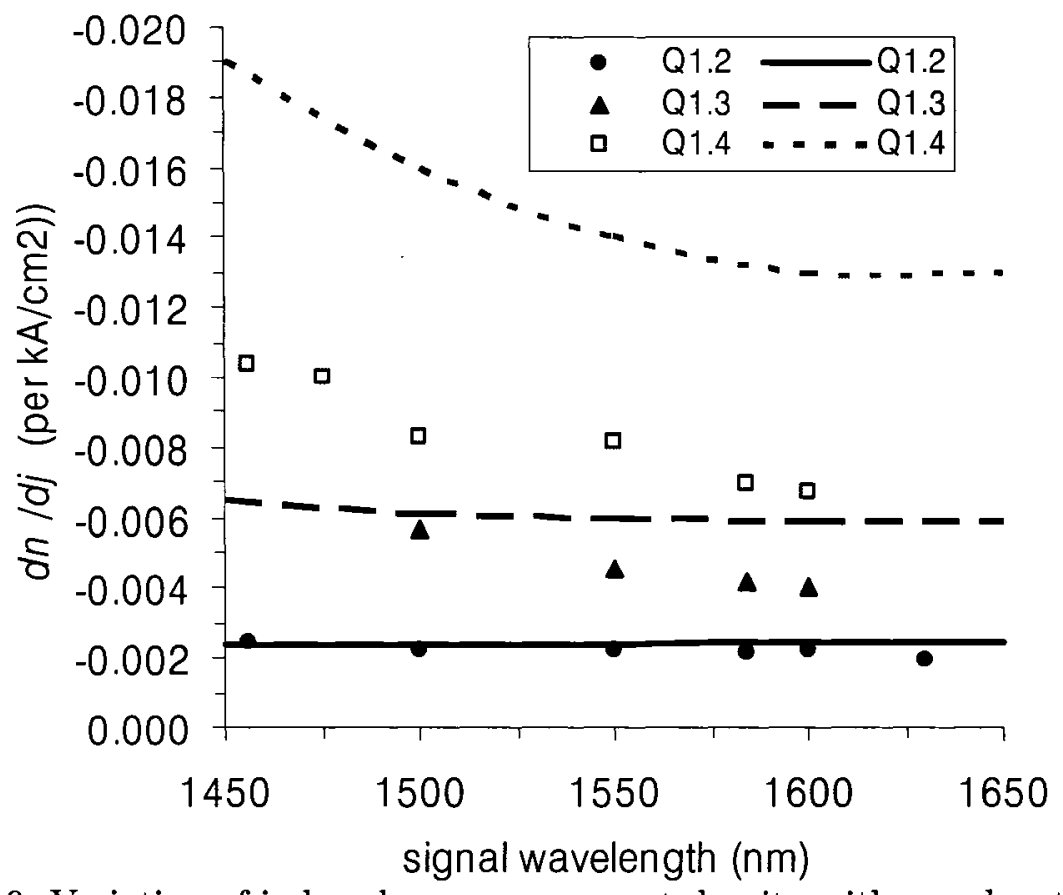

Figure 4-9 Variation of index change per current density with wavelength from theory (lines) and from measurements of MZ interferometer fringes for devices on MOCVD grown $\mathrm{Q}=1.4 \mu \mathrm{m}, 1.3 \mu \mathrm{m}$, and $1.2 \mu \mathrm{m}$ wafers (points).

To enable a comparison of the measured index modulation with theory presented in Figure 4-9, the theoretical results in Figure 4-5 for the three InGaAsP alloys were used to calculate the predicted $d n / d j$ for our waveguide material and geometry. In each case, the current density required to generate the corresponding carrier concentration in Figure 4-5 was estimated using (1) and assuming lifetimes of 3, 7, and $17 \mathrm{~ns}$ respectively for the $\mathrm{Q}=1.2,1.3$, and $1.4 \mu \mathrm{m}$ alloys. These lifetimes were determined from measured switching speeds, as described later. The experimental and theoretical values of $d n / d j$ in Figure 4-9 were determined for a carrier concentration of $2.5 \times 10^{17}$ $\mathrm{cm}^{-3}$.

This is the first reported comparison of experimental and theoretical values of index change for different InGaAsP alloys using the BGS model of Botteldooren and Baets [60]. The index change due to bandgap shrinkage $[58,59]$ is opposite in sign and hence partially cancels the index shift caused by bandfilling. The total calculated index change can therefore be very sensitive to the accuracy of the models used to calculate the two counteracting effects. Additionally, several approximations were made in our 
device model, such as neglecting carrier diffusion. A more detailed analysis of the modal field overlap with the carrier distribution for a few specific cases indicates that our simple model overestimates the $d n / d j$ values derived both from experiment and from the theoretical curves of Figure 4-5 by less than a factor of two. This is discussed further below.

Nevertheless, while the experimentally determined values of $d n / d j$ are smaller than theoretical values, the experimental results are in good qualitative agreement with the theoretical predictions. As expected, the values of $d n / d j$ are largest for the smallest bandgap $(\mathrm{Q}=1.4 \mu \mathrm{m})$ material, and the values increase as the signal wavelength approaches the band edge of the material, with the $\mathrm{MZ}$ device on the $\mathrm{Q}=1.4 \mu \mathrm{m}$ wafer exhibiting the largest index modulation slope. In contrast, the theoretical curve for $\mathrm{Q}=1.2 \mu \mathrm{m}$ InGaAsP alloy shows a slight decrease in $d n / d j$ as the signal wavelength decreases. This is because the signal wavelengths are far from the band edge for this alloy and so bandfilling and BGS have a smaller effect compared with free carrier absorption, for which index change is proportional to the square of the wavelength as given by equation (3-1). Experimental results show no such decrease in $d n / d j$ with decreasing wavelength, suggesting that bandfilling and BGS are a bigger effect relative to the effect of free carrier absorption than theory predicts.

However absorption losses are slightly higher for this larger bandgap quaternary. The measured difference in insertion loss between a $2 \mathrm{~mm}$ straight waveguide on $\mathrm{Q}=1.4 \mu \mathrm{m}$ and $\mathrm{Q}=1.3 \mu \mathrm{m}$ material is only about $1 \mathrm{~dB}$ for light within the C-band.

Similar MZ interferometer fringe measurements were also performed on devices fabricated on the MBE grown wafers. The slopes $d n / d j$ were extracted from the MZ interferometer measurements for all of the wafers and are presented in Table 4-1. The value of $d n / d j$ is about three times larger for the otherwise identical devices on MBE than MOCVD wafers. This suggests that the MBE material has a longer lifetime which, from equation (4-6) allows a larger carrier concentration to collect in the core for a given applied current. This is further supported by characterization of RW-DOS devices. 
Table 4-1 Material index change versus current density values from MZ interferometer fringe data with TE polarized broadband light

\begin{tabular}{|c|c|c|c|}
\hline & \multicolumn{3}{|c|}{$\mathrm{dn} / \mathrm{dj}\left(\mathrm{kA} / \mathrm{cm}^{2}\right)^{-1}$} \\
\hline Wafer growth & $\mathbf{Q}=\mathbf{1 . 2} \mu \mathrm{m}$ & $\mathbf{Q}=1.3 \mu \mathrm{m}$ & $\mathbf{Q}=1.4 \mu \mathrm{m}$ \\
\hline MBE & -0.004 & -0.015 & -0.025 \\
\hline MOCVD & -0.0022 & -0.0047 & -0.0086 \\
\hline
\end{tabular}

Fabry-Perot (FP) interferometers formed by simple straight waveguides with a $1.4 \mathrm{~mm}$ long injection electrode, and terminated by the cleaved facets, were fabricated to elucidate the effects of diffusion on the induced index change. Similarly to MZ devices, refractive index $n$ can be determined as a function of applied current density $j$ from FP fringe oscillations. The experimentally determined index modulation is shown in Figure 4-10 for devices with waveguide widths of $5 \mu \mathrm{m}, 4 \mu \mathrm{m}$, and $3 \mu \mathrm{m}$ in $\mathrm{TE}$ polarization. Results in TM polarization are identical to those shown here. Poor transmission through the $5 \mu \mathrm{m}$ waveguide limited its measurement range. The $d n / d j$ values increase with waveguide width suggesting that the wider optical mode profile has a better overlap with the injected carrier distribution, and thereby allows a larger modulation in the modal effective index. In addition to carrier diffusion, narrower waveguides are also more adversely affected by current spreading between the electrode contact and the waveguide core layer, which can reduce the mode overlap with the carrier distribution even further.

Incidentally, fabrication on the MBE wafers resulted in waveguides with slanted side walls and waveguide widths $\sim 0.5 \mu \mathrm{m}$ less than the designed width. Thus in the comparison of $\mathrm{d} n / \mathrm{d} j$ between MOCVD and MBE wafers, the MBE results may be slightly lower than expected for nominal waveguide widths. 


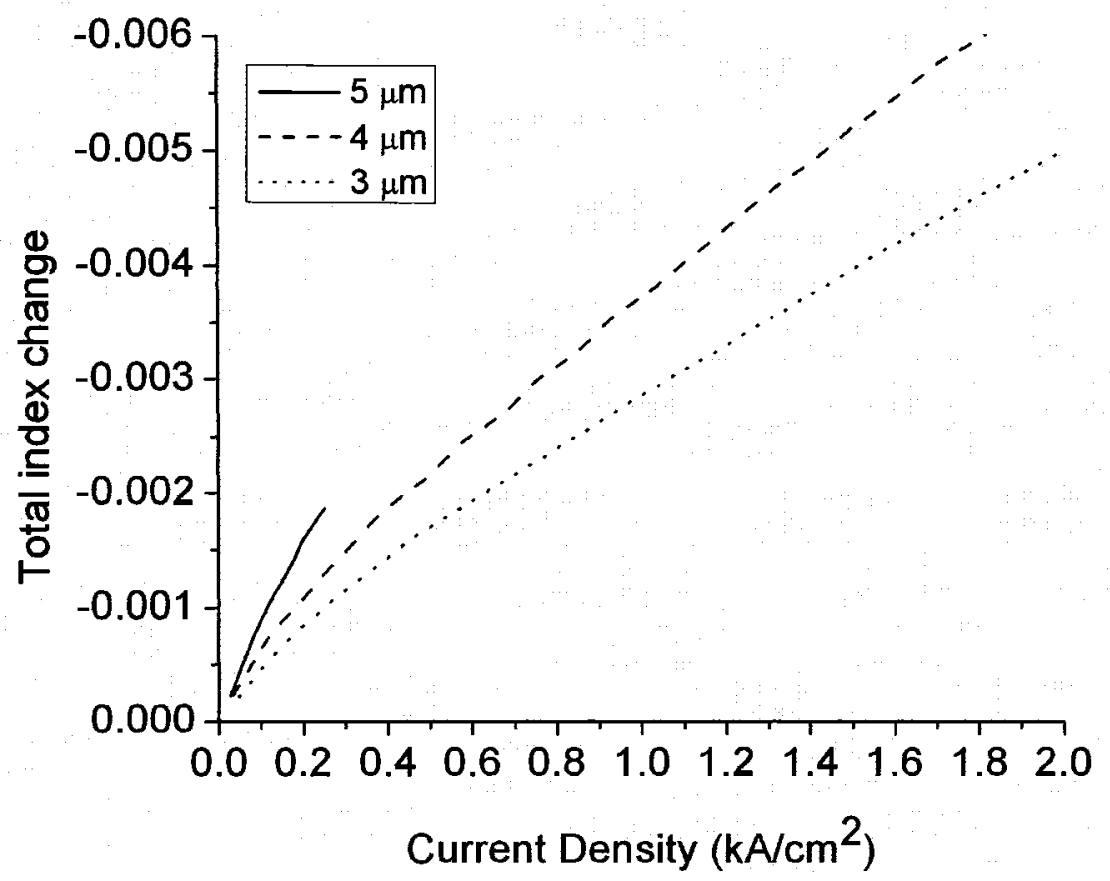

Figure 4-10 Index change versus current density calculated from Fabry-Perot (FP) fringes measured using TE polarized broadband light for devices on $\mathrm{Q}=1.4$ $\mu \mathrm{m}$ wafers with waveguides widths $5 \mu \mathrm{m}, 4 \mu \mathrm{m}$, and $3 \mu \mathrm{m}$.

\subsection{Correction for Carrier Diffusion}

Carrier diffusion results in a reduced peak carrier concentration and a broadened index profile as shown in Figure 4-6. As mentioned previously, the optical model assumes that the material index change is constant and confined beneath the waveguide ridge. Thus by not considering carrier diffusion, the values of index change and carrier concentration determined from measurements, such as those plotted in Figure 4-9, are overestimated.

A correction for carrier concentration can be derived from the diffusion equation when concentration gradients in the vertical direction can be neglected, which is a reasonable approximation for a thin core layer with high energy barriers to prevent escape of electrons and holes. For a ridge of width $w$, the concentration $N$ at the center of the ridge is: 


$$
N=\frac{j \tau}{q d}\left(1-e^{-w / 2 L_{D}}\right)
$$

where $L_{D}=\sqrt{D_{a} \tau}$ is the diffusion length, $\tau$ is the carrier lifetime, and $D_{a}$ is the ambipolar diffusion coefficient for the quaternary. Values of $D_{a}$ were calculated from the mobilities given in [60].

The correction for index change requires a calculation of the mode overlap of the carrier distribution with the optical mode profile. The mode profile depends upon the refractive index profile, which is related to the carrier distribution. An accurate simulation would thus include the dependence of lifetime on carrier density and the evolution of the waveguide mode with changes in material index. These require dynamic simulations that are not within our current capabilities. An estimate may be calculated using the carrier distribution from drift diffusion simulations at a given current density and the mode profile of the ridge waveguide assuming no index change. Such an estimate revealed that the simpler model overestimates index change. A calculation of the extreme case where the index change occurs throughout the core layer provides an upper limit on the required correction factor.

Calculated values of index change based on $\mathrm{MZ}$ measurements for the three InGaAsP alloys at $1550 \mathrm{~nm}$ are plotted as solid points in Figure 4-11 for comparison with theory. They are plotted at the carrier concentration corresponding to $0.1 \mathrm{kA} / \mathrm{cm}^{2}$ applied current density, as related by equation (4-6). By using equation (4-8) and assuming lifetimes of $3 \mathrm{~ns}, 7 \mathrm{~ns}$, and $17 \mathrm{~ns}$ for the $\mathrm{Q}=1.2 \mu \mathrm{m}, 1.3 \mu \mathrm{m}$, and $1.4 \mu \mathrm{m}$ alloys respectively, calculated values of carrier concentration are reduced and the experimental points shift to the left according to the arrow in the plot. Using the extreme cases described above of index change occurring only under the ridge and throughout the core layer, a range of index change values is indicated with shaded rectangles. As seen in Figure 4-11, this range of index change values straddles the theoretical data. Thus while there is a discrepancy between the values of index change calculated from measurements and determined theoretically, there is qualitative agreement in the relative values amongst quaternaries, and preliminary calculations of correction factors 
reduce this discrepancy. Uncertainty as to the accuracy of the idealized model limits comparison with the real device and so a more detailed analysis is unwarranted.

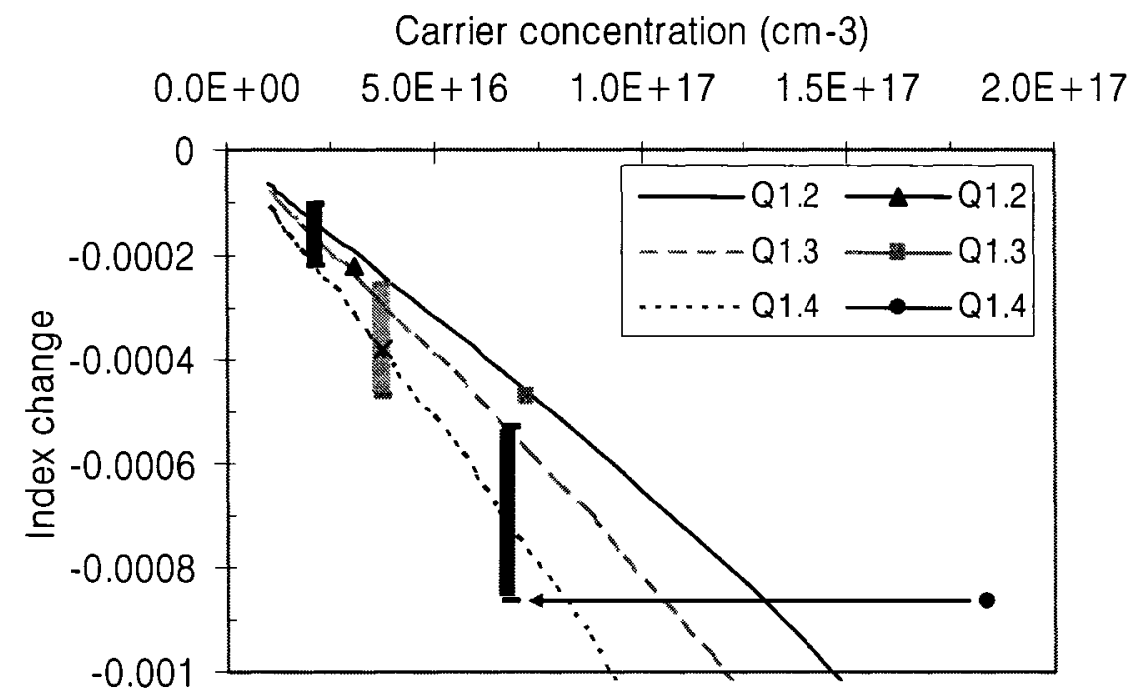

Figure 4-11 Modeled (lines) and measured (points) values of index change at $1550 \mathrm{~nm}$ versus carrier concentrations for MOCVD-grown InGaAsP of bandgap (Q) $1.4 \mu \mathrm{m}, 1.3 \mu \mathrm{m}$, and $1.2 \mu \mathrm{m}$.

\subsection{Quantum Well Structures}

To characterize carrier injection in quantum well (QW) structures, Mach-Zehnder (MZ) and Fabry-Perot (FP) devices on the QW wafers were tested under forward bias. The wafers were designed to have a band edge at $1420 \mathrm{~nm}$ to be characterized at $1550 \mathrm{~nm}$, however, the transmission data of Figure 4-12 shows onset of absorption for wafers RQWa, RQWb, and 3SQW to be around $1570 \mathrm{~nm}, 1580 \mathrm{~nm}$, and $1540 \mathrm{~nm}$ respectively, resulting in very low transmission at $1550 \mathrm{~nm}$. Photoluminescence measurements at room temperature with a $980 \mathrm{~nm}$ laser diode source show emission peaks between 1475 and $1478 \mathrm{~nm}$ for all three wafers indicating the room temperature band gap wavelength was approximately $50 \mathrm{~nm}$ to the red of the original specification. Thus, the devices were characterized with a tunable laser at $1600 \mathrm{~nm}$ and $1630 \mathrm{~nm}$. Although transmission through the QW wafers was very poor in the C-band, 
measurements on MZ were taken using an EDFA source to directly compare the performance of carrier injection in QW and bulk materials.

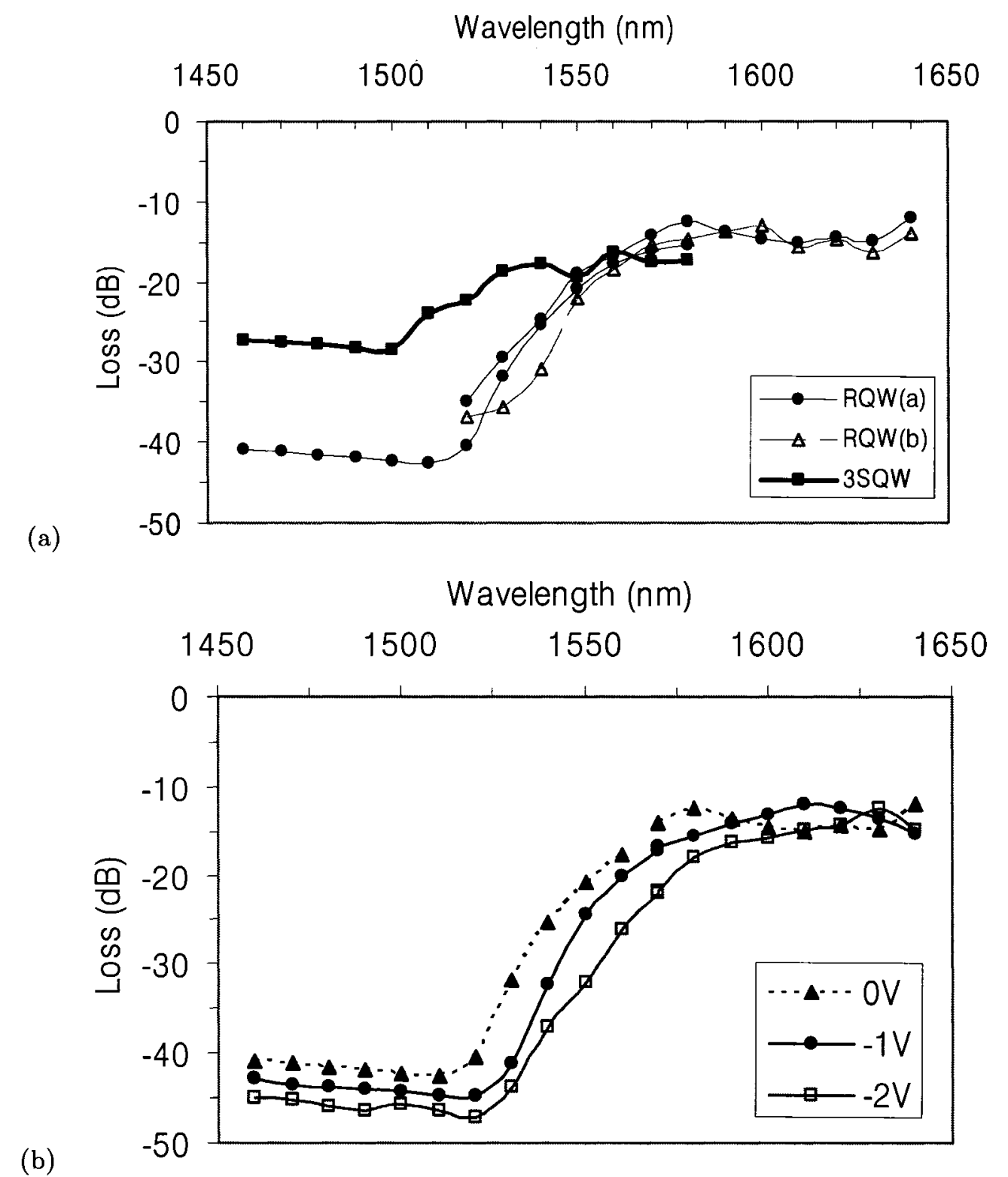

Figure 4-12 Spectral transmission of (a) the three QW wafers corresponding to the designs in Figure 3-12, and (b) RQWa with applied reverse bias of $0 \mathrm{~V},-1 \mathrm{~V}$, and $-2 \mathrm{~V}$. 


\subsubsection{C-band broadband measurements}

Experimental data relating index change with current density were collected using a broadband EDFA source. Values of $\mathrm{d} n / \mathrm{d} j$ were then taken as the linear coefficient of a fitted function relating index change to current density. These values are shown in comparison with the values for the bulk MOCVD and MBE wafers in Table 4-2. In general, the QW wafers showed comparable $\mathrm{d} n / \mathrm{d} j$ to the bulk MBE wafers, however, the devices had large transmission loss and large polarization dependent loss.

Table 4-2 Index modulation of modal index per applied current density $\mathrm{d} n / \mathrm{d} j$ in $\mathrm{kA}^{-1} \mathrm{~cm}^{2}$ for MOCVD and MBE grown wafers.

\begin{tabular}{|l|l|l|l|}
\hline $\mathrm{dn} / \mathrm{dj}$ (mode) & MOCVD TE & MBE TE & MBE TM \\
\hline $\mathbf{Q}=\mathbf{1 . 2} \mu \mathrm{m}$ & $-1 \times 10^{-3}$ & $-2 \times 10^{-3}$ & $-3 \times 10^{-3}$ \\
\hline $\mathbf{Q}=1 . \mathbf{3} \mu \mathrm{m}$ & $-2 \times 10^{-3}$ & $-7 \times 10^{-3}$ & $-6 \times 10^{-3}$ \\
\hline $\mathbf{Q}=1.4 \boldsymbol{\mu m}$ & $-3 \times 10^{-3}$ & $-14 \times 10^{-3}$ & $-15 \times 10^{-3}$ \\
\hline $\mathbf{R Q W a}$ & & $-9 \times 10^{-3}$ & $-5 \times 10^{-3}$ \\
\hline RQWb & & Power too low & $-1 \times 10^{-3}$ \\
\hline 3SQW & & $-5 \times 10^{-3}$ & $-2 \times 10^{-3}$ \\
\hline
\end{tabular}

Transmission through the three wafers was polarized, with TE polarization highly attenuated as expected since absorption in a quantum well superlattice is anisotropic. Absorption of TM polarization is weaker due to its reduced heavy hole interaction [77]. In wafer RQWb, which has the smallest bandgap, transmission of TM and TE polarization were comparable (both low), but in wafer 3SQW, which has the largest bandgap, TM transmission was about $10 \mathrm{~dB}$ better than $\mathrm{TE}$.

\subsubsection{L-band single wavelength measurements}

Transmission is better in the L-band, above the absorption edge of the QW wafers. Calculated values of index change from MZ interferometers (with $3.2 \mathrm{~mm}$ electrodes) and FP fringes from long straight waveguides (with $5.2 \mathrm{~mm}$ electrodes) were consistent, except for one wafer. The long FP structure provides better resolution, while the MZ structure provides measurements of index change over a larger range, as seen below. Results are shown in Figure 4-13. 


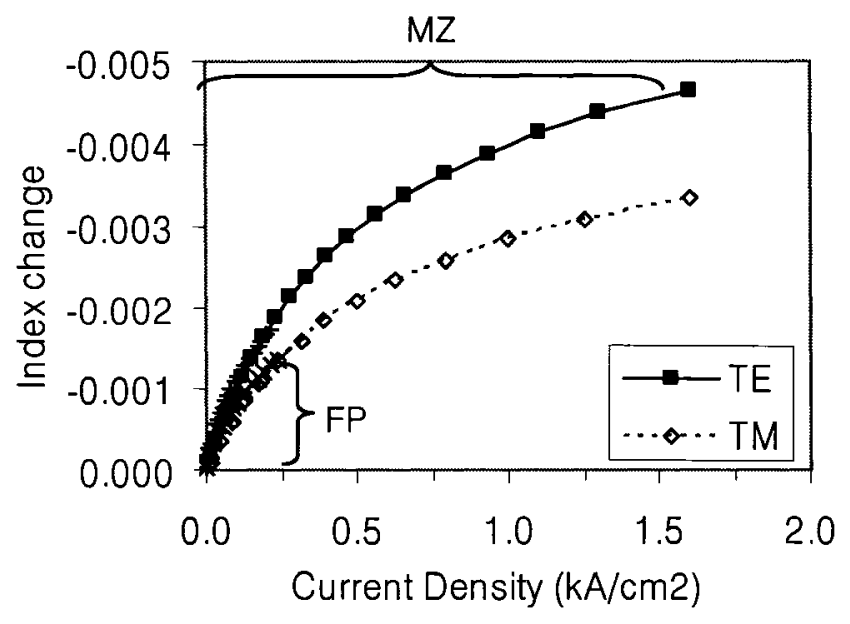

(a)

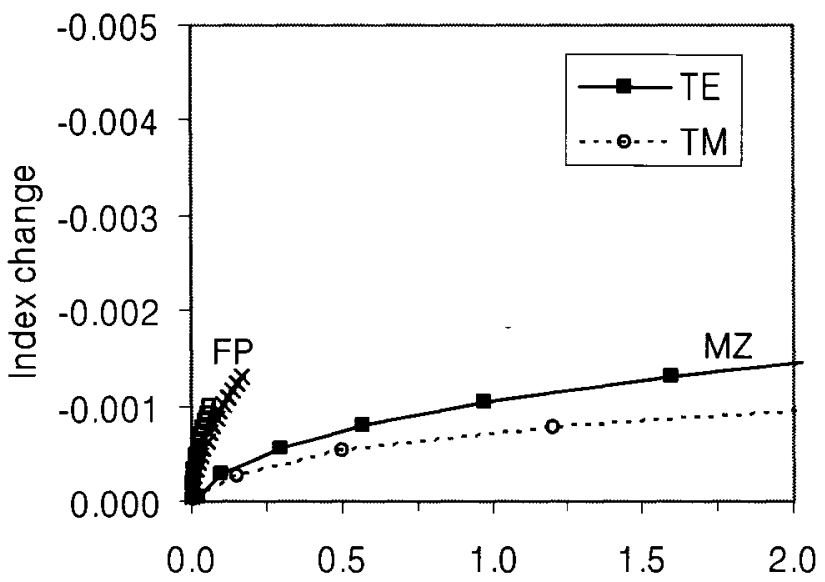

(b)

Current Density $(\mathrm{kA} / \mathrm{cm} 2)$

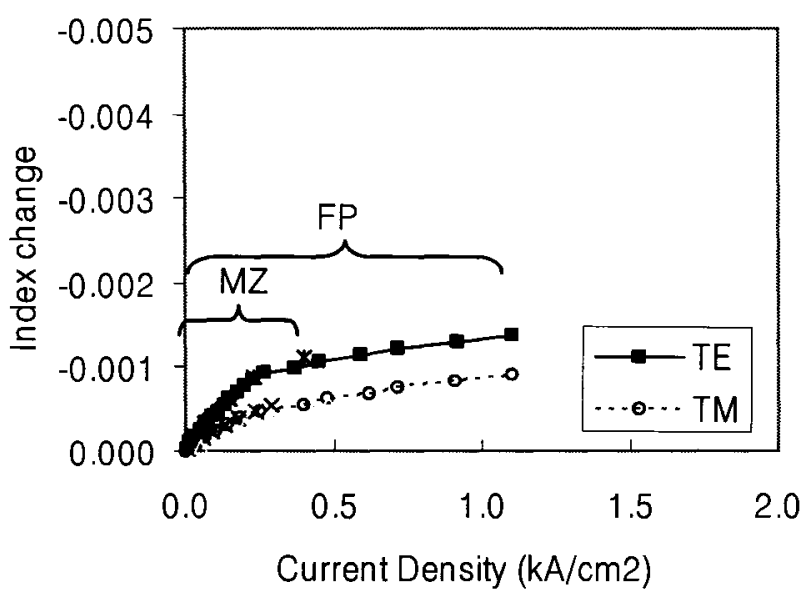

Figure 4-13 Plots of index change versus current density calculated from observed FP and MZ interference fringes for the QW wafers (a) RQWa (b) RQWb and (c) 3SQW. The source was a tunable laser at $1600 \mathrm{~nm}$. 
FP and MZ results were effectively the same for wafer RQWa and 3SQW; wafer RQWb was the only wafer for which the MZ and FP results were inconsistent. Its MZ results give a much smaller index change with applied currrent density, while the FP results give larger index changes comparable to the results of wafer RQWa. The MZ results could not be verified by measurements on duplicate structures because there was only one working $\mathrm{MZ}$ device on that chip, thus the one available MZ device is suspect, given that the switching performance and FP results are similar to those for wafer RQWa.

The index changes calculated from FP fringes from short straight waveguides (with 1.8 $\mathrm{mm}$ electrodes) were larger than values calculated from the $\mathrm{MZ}$ interferometers or the longer straight waveguides with $5.22 \mathrm{~mm}$ electrodes. Additionally, some of the short waveguides exhibited a "beat pattern" as two different periods appeared to be present in the fringes. This may have been due to the presence of a higher order waveguide mode. For further comparisons, I use the MZ results because they cover a larger range of current density than the FP results.

The experimentally measured index change versus current density for wafer RQWa is similar to though smaller than previously published results [78]. That paper has three recommendations for enhancing carrier induced index changes in quantum wells: a larger number of wells, a smaller bandgap barrier, and a larger bandgap well material. The latter two conditions, in effect, realize a structure with a "small band discontinuity between the well and the barrier" [78]. Thus the suggested quantum well structure in that paper is 15 periods of $Q=1.55 \mu \mathrm{m}$ InGaAsP well and $Q=1.4 \mu \mathrm{m}$ InGaAsP barrier. In contrast, the $\mathrm{QW}$ structures that we fabricated had a large energy step between well and barrier since it was designed for reverse bias electrooptical actuation. Thus, carrier injection switching performance may be improved by a redesign of the quantum well materials.

The nonlinear relationship of the index change with current density makes it difficult to characterize $\mathrm{dn} / \mathrm{dj}$ using a single number, so for comparison with the bulk materials, the data for bulk InGaAsP are plotted along with the results for QW wafer RQWa in Figure 4-14. The $\mathrm{QW}$ material has an initial slope similar to that of bulk $\mathrm{Q}=1.3 \mu \mathrm{m}$ 
InGaAsP, but appears to have a higher degree of nonlinearity. In general, the QW results show the following trends: values of $\mathrm{dn} / \mathrm{dj}$ were $10-20 \%$ smaller at $1630 \mathrm{~nm}$ than at $1600 \mathrm{~nm}$, and they were slightly larger for TE than for TM polarization.

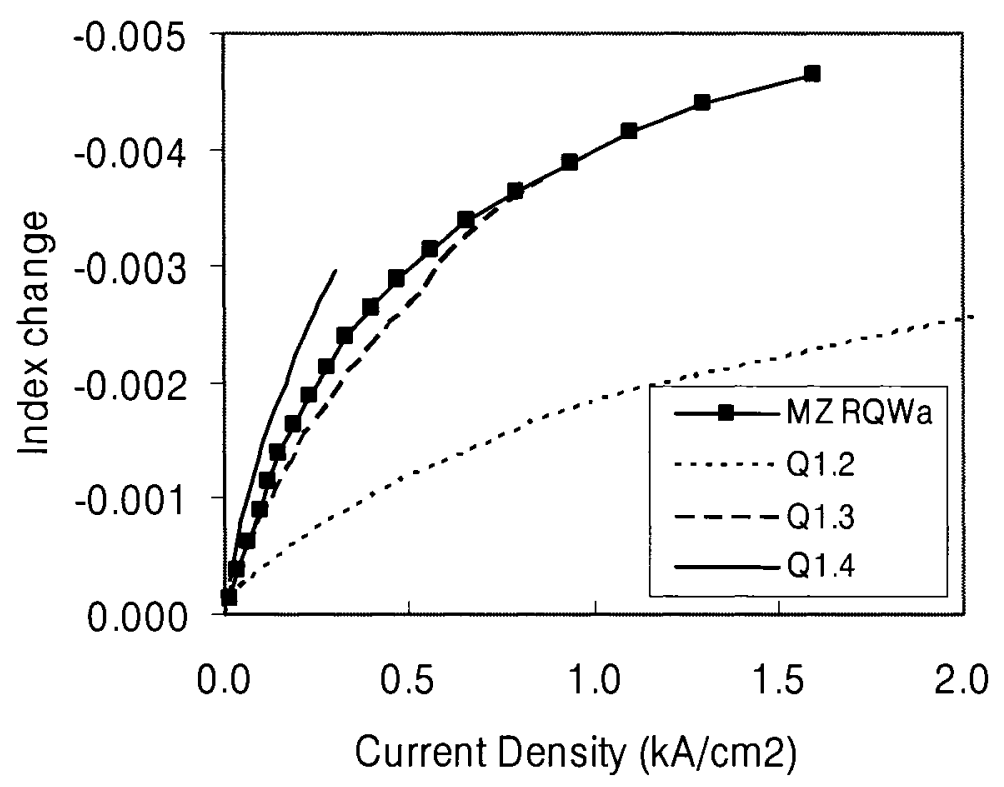

Figure 4-14 Plots of index change versus current density calculated from observed MZ interference fringes for QW wafer RQWa in comparison with bulk wafers in TE polarization.

\subsection{Summary}

Using Mach-Zehnder and Fabry-Perot interferometer devices, we have performed a comparison of measured index change against modeling according to Bennett et al. [59], Weber [58], and Botteldooren and Baets [60] for three different InGaAsP alloys. Although the effects of diffusion were not included in the modeling, the experimental and theoretical results agree qualitatively. In particular, the refractive index change per applied current density increases as the signal wavelength approaches the band edge of the material. Quantum well structures demonstrate comparable magnitudes of index change per applied current density, even though they were not optimized for operation by current injection. The results of our studies of carrier injection are 
applied to our RW-DOS design in the next chapter and to further carrier injection devices later. 


\section{Chapter 5}

\section{CHARACTERIZATION OF RW-DOS DEVICES}

\subsection{Device Design Parameters}

Device design was further optimized through experimental characterization of many variations of DOS devices. Figure 5-1(a), shows the variation of the output power (normalized to the input power) with $\Delta \mathrm{n}$ in the two DOS switch arms, calculated for three different branching angles $\alpha$. The decreased contrast ratio and higher insertion loss for larger angles are explained by high bend losses at the $\mathrm{Y}$-junction. The measured output powers for the biased and unbiased DOS arms as a function of the applied current for three different branching angles are shown in Figure 5-1(b). These results are in good agreement with simulations for the DOS switching contrast vs. the branching angle. The measured switching contrast ratio is $\sim 25 \mathrm{~dB}$, limited by noise in the experimental setup. The DOS operation was wavelength independent over a $50 \mathrm{~nm}$ bandwidth around $1550 \mathrm{~nm}$.

The variations in electrode offset described previously in Chapter 3.4 did not significantly affect switching performance and so the nominal value of $1 \mu \mathrm{m}$ was used as a standard in subsequent designs. 


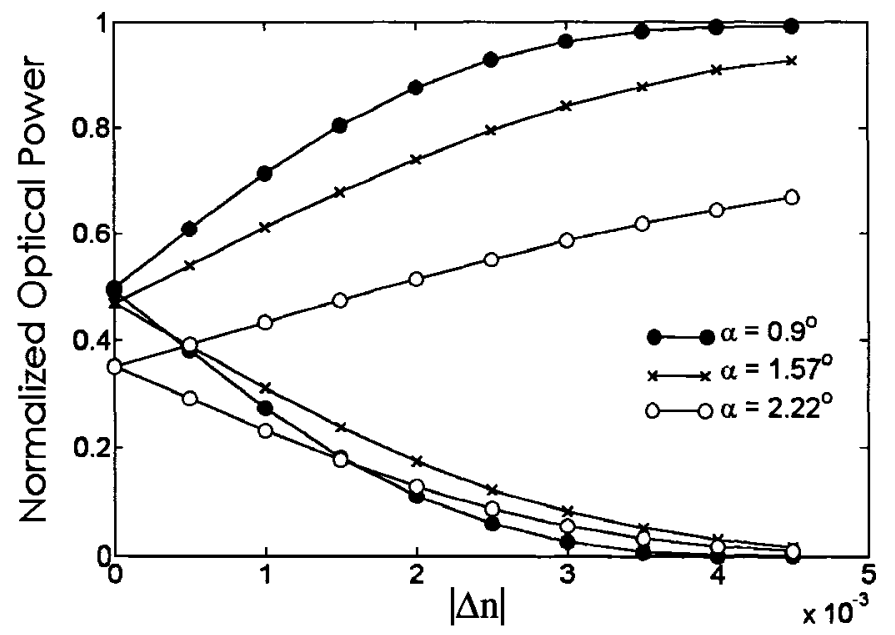

(a)

(b)

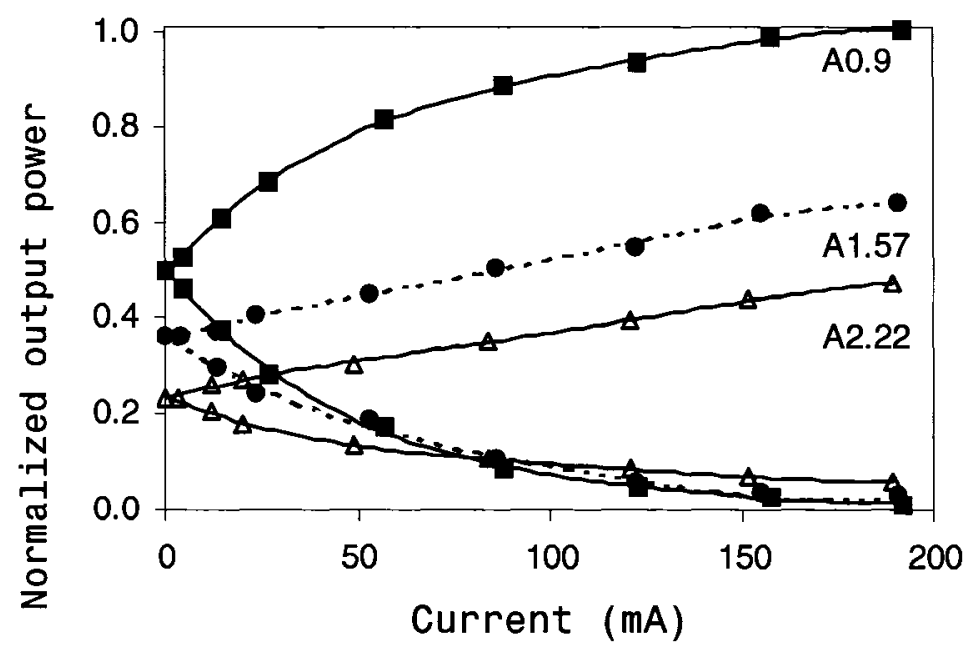

Figure 5-1 (a) Calculated output power in the biased (bottom) and unbiased (top) RW-DOS arms as a function of the induced refractive index change for three branching angles, and (b) the corresponding output power measured in fabricated RW-DOS devices as a function of bias current.

Waveguide loss was calculated using equation (4-3) and the measured minimum $I_{m i n}$ and maximum $I_{\max }$ intensities of Fabry Perot interferometer fringes from a $2 \mathrm{~mm}$ long straight waveguide. The propagation loss for TE and TM polarizations through the straight waveguide were 2.2 and $2.5 \mathrm{~dB}$ respectively. The best total output power including both polarizations coupled out of a RW-DOS was about $400 \mu \mathrm{W}$. Given an input power of $10 \mathrm{~mW}$, the total loss, including fiber to waveguide coupling and device loss, is thus about $14 \mathrm{~dB}$. 


\subsection{Electrical Isolation of Electrodes}

Practical aspects of fabrication of the DOS affect optical performance and play a role in the development of viable devices. For example, the DOS electrodes are separated by less than $1 \mu \mathrm{m}$ at the closest point, a unique feature of our devices necessitating a means of electrical isolation between the two arms.

Electrical isolation of neighbouring devices is an important issue in the development of semiconductor integrated circuits in general. Isolation reduces crosstalk and leakage current, and most importantly it reduces parasitic capacitances that may slow down a device. Effective isolation allows devices to be placed close together, thus reducing overall chip size. Field effect transistors and lasers are practical examples of devices that routinely require electrical isolation. In the past, such isolation has been achieved through both ion implantation $[79,80]$ and the processing of shallow [81] or deep trenches [82].

Electrical isolation within an optical device is less common, and it introduces different constraints. Namely the isolation mechanism must function on a sub-micron scale and it cannot perturb the underlying guided optical fields. Trenches have previously been reported for use in isolating contacts in an optical switch [83], however not for this particular DOS design. As well, the performance of trench and implant isolation have not previously been compared and assessed.

Ion implantation maintains the planarity of a device surface, which is important if there are subsequent processing steps. Implantation also perturbs stress fields less than a trench, as further discussed below. However, the lateral diffusion, or straggle, of ions during implantation shifts the edges of the isolation region from the edge of the implant mask. Another disadvantage of implantation isolation is that it is a multi-step process that requires careful design of parameters, such as mask thickness and implantation energies. However, implant isolation is well established and well understood. Therefore the implant process can be easily optimized to minimize the effects of straggle by using appropriate ions, implant energies, multiple implants, and implant angle. 
In the case of the DOS, the trenches are formed in the same etch step as the device ridge waveguides, as shown in Figure 5-2. Removing the step of ion-implantation also eliminates the need for the mask layer that is used as a window during ion implantation. These reduce device fabrication time and cost. An air filled trench has lower capacitance than semiconductor material, and so physical isolation with the trench is ideal for high-speed devices. However, an isolation trench introduces non-planarity of the device surface, complicating subsequent deposition of metal layers. In our case, the DOS ridge waveguides are non-planar, so planarization is already an essential step in device fabrication. Undercutting, which is inherent to wet etching, can distort the edges of the isolation region. This process bias must be taken into account in the mask design. Finally, the trench can contribute stresses, which have been shown to affect carrier mobility [84], material bandgap, and refractive index. This leads to leakage currents around the bottom corners of the trench [82].

lon-implant isolation

0

2

4

6

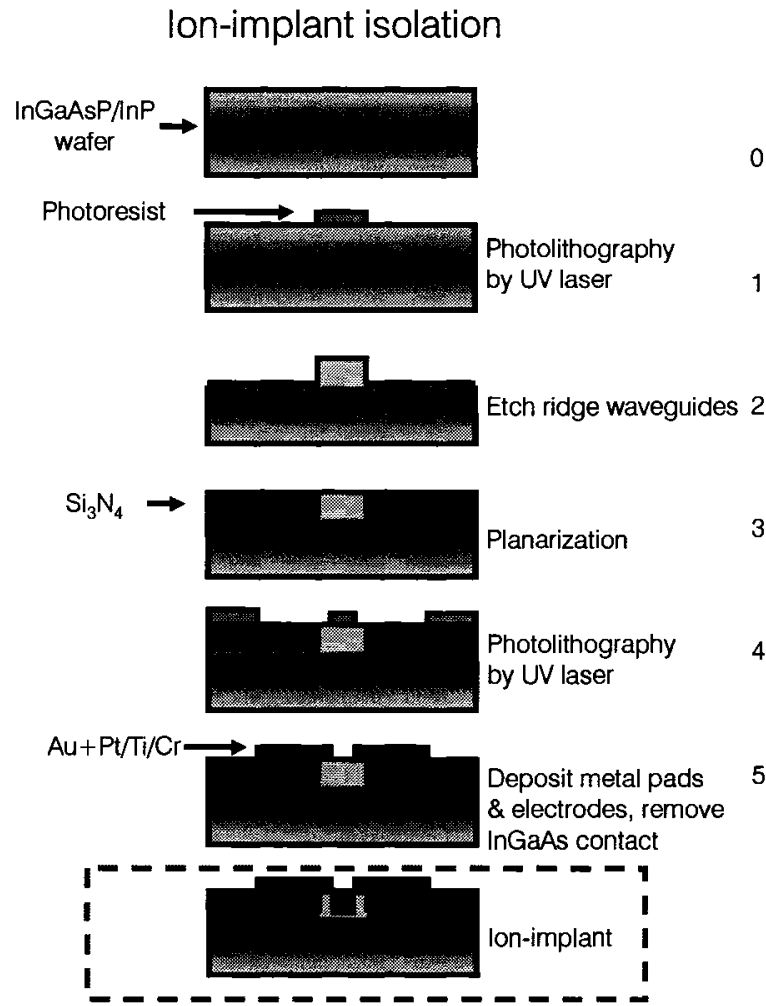

(a)
Trench isolation
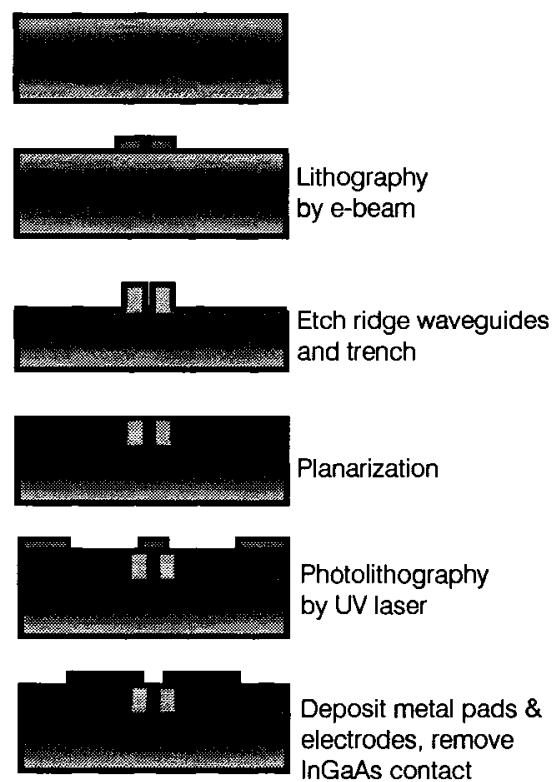

Photolithography by UV laser

Deposit metal pads \& electrodes, remove InGaAs contact

(b)

Figure 5-2 Comparison of fabrication steps of electrode isolation by (a) ionimplant and (b) trench isolation. 
We compare implant and trench isolation of two electrodes separated by a gap of less than $1 \mu \mathrm{m}$. Hole carrier distributions are calculated and optical beam propagation simulations are presented for both methods of electrical isolation. Electrical and optical experimental results are also presented.

\subsubsection{Electrode Isolation}

The electrodes in one set of switches are electrically isolated by an oxygen ion $\left(\mathrm{O}^{+}\right)$ implant as illustrated in Figure 3-13 (b). Isolation is accomplished through the creation of deep-level centers that trap free carriers [80]. The electrodes are composed of a metal stack of $500 \AA \mathrm{Ti} / 600 \AA \mathrm{Pt} / 5000 \AA \mathrm{Au}$, which also serves as a self-aligned shadow mask for the curved edges of the implantation region.

The ion implant parameters are calculated using ProfileCode by Implant Sciences Corporation. The results of the ion concentration calculations are presented in Figure $5-3$, which show a relatively flat implantation profile down to a depth of about $600 \mathrm{~nm}$, the first $100 \mathrm{~nm}$ being a silicon nitride $\left(\mathrm{Si}_{3} \mathrm{~N}_{4}\right)$ layer that reduces straggle. After removing the InGaAs contact layer in between DOS branches, samples are irradiated with three consecutive implants of $85 \mathrm{keV}, 175 \mathrm{keV}$ and $350 \mathrm{keV} \mathrm{O}^{+}$to doses of $7 \times 10^{12} / \mathrm{cm}^{2}, 9.5 \times 10^{12} / \mathrm{cm}^{2}$, and $2 \times 10^{13} / \mathrm{cm}^{2}$, respectively. The samples are then annealed in $\mathrm{N}_{2}$ for 5 minutes at a temperature of $350{ }^{\circ} \mathrm{C}$. This implant is designed such that the summation of the three implantation processes gives a flat O-doping profile of $5 \times 10^{17} / \mathrm{cm}^{3}$ along the first $500 \mathrm{~nm}$ of InP material and decays by two orders of magnitude in the following $250 \mathrm{~nm}$. Simulations show a lateral straggle of about $120 \mathrm{~nm}$. 


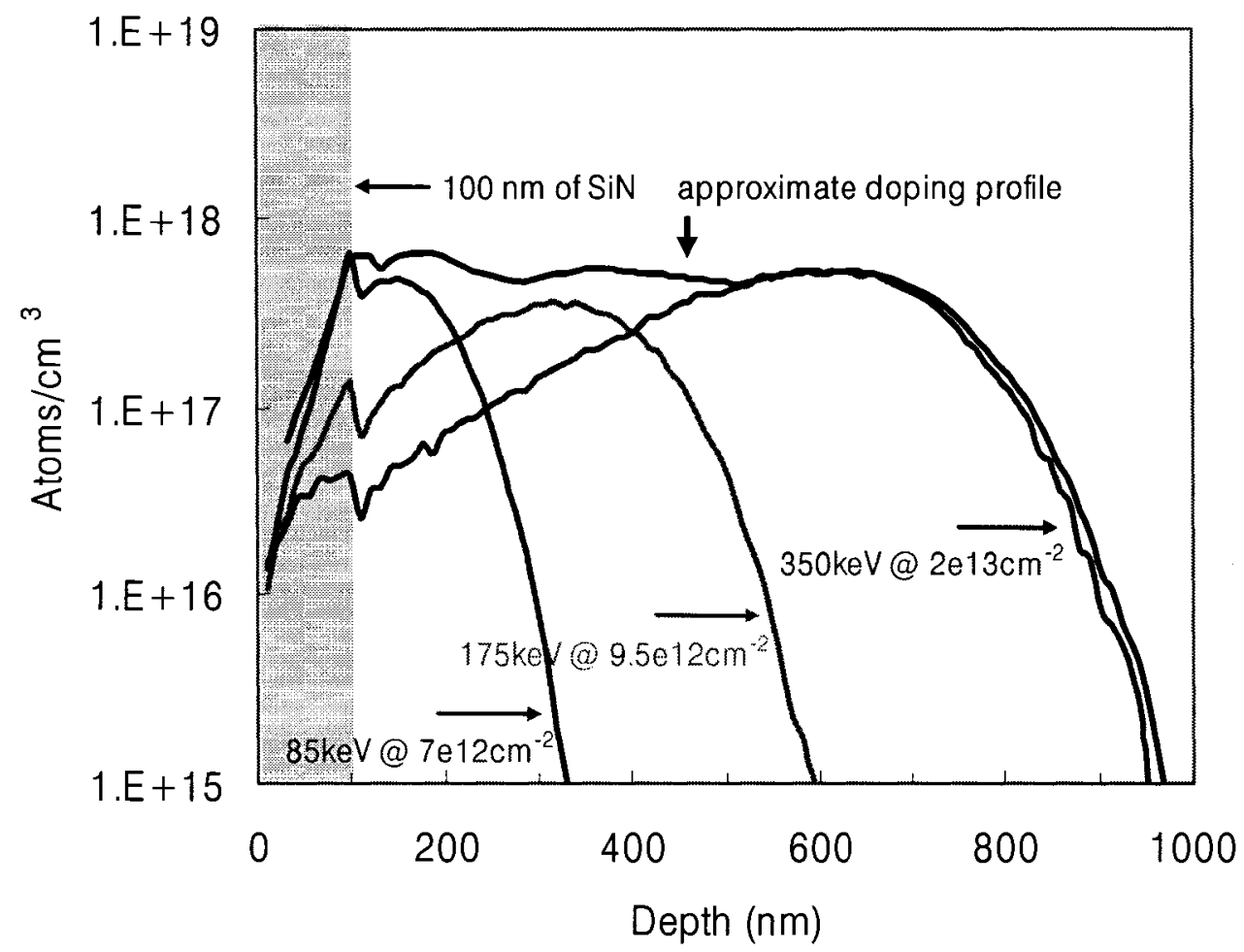

Figure 5-3 Calculated ion implantation concentrations versus depth.

Another set of switches is electrically isolated by the presence of a $0.5 \mu \mathrm{m}$ wide trench between the contacts as illustrated in Figure 3-13 (top left). The trench extends from the start of the contacts to the vertex of the Y-branches, a distance of about $300 \mu \mathrm{m}$ as shown in Figure 3-13 (bottom left). The trench is patterned along with the waveguides by electron-beam (e-beam) lithography and etched by inductively coupled plasma reactive ion etching (ICP-RIE) followed by a wet etch down to the etch-stop layer. Scanning electron microscope (SEM) images of the trench are shown in Figure 5-4. A trench width of $0.52 \mu \mathrm{m}$ was measured, and the trench depth was determined to be within $20 \mathrm{~nm}$ of the etch stop. 
(a)
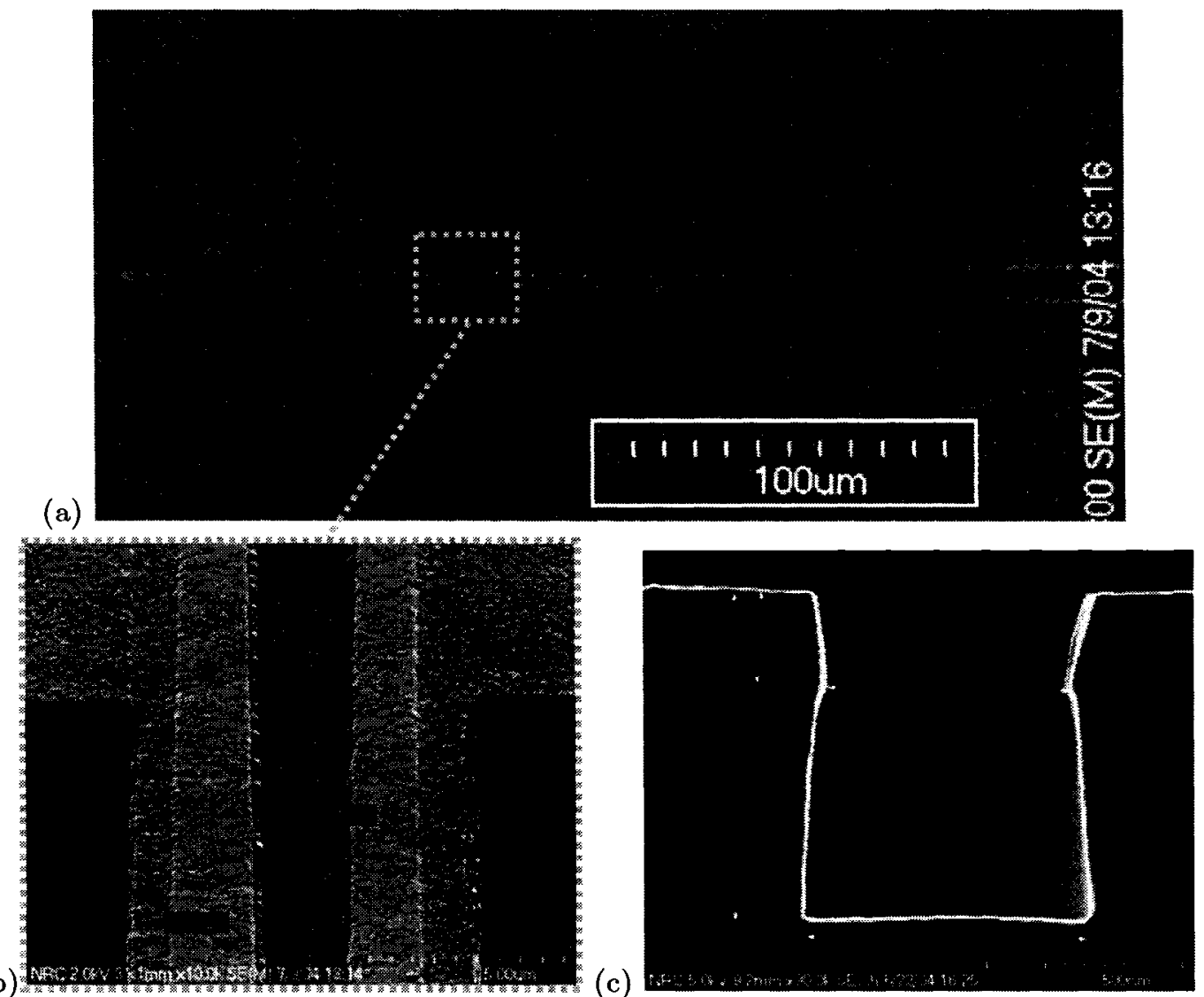

Figure 5-4 SEM images of a DOS isolation trench (a) from the top, (b) magnification of the area in the dashed box, and (b) in cross section.

\subsubsection{Numerical Simulations}

The electrical performance for both structures is simulated with $2 \mathrm{D}$ drift-diffusion calculations using mobilities from Sotoodeh et al. [71] and a recombination time of 1 ns. The ion implant is modeled as constant across the implantation region with sharp cutoffs at the implant mask boundaries. This is a reasonable approximation and would not affect carrier diffusion profiles as the implant straggle is only $0.12 \mu \mathrm{m}$, which is an order of magnitude smaller than carrier diffusion lengths. Figure 5-5 (a) shows the simulation results of the hole concentration in the core layer for devices with ion implant and with physical trench isolation. Hole distributions show similar quantities of injected carriers in both cases, thus it is expected that the magnitude of refractive index modulation due to carrier injection be similar in both cases, approximately $10^{-2}$ at the maximum of the carrier distribution [67]. 

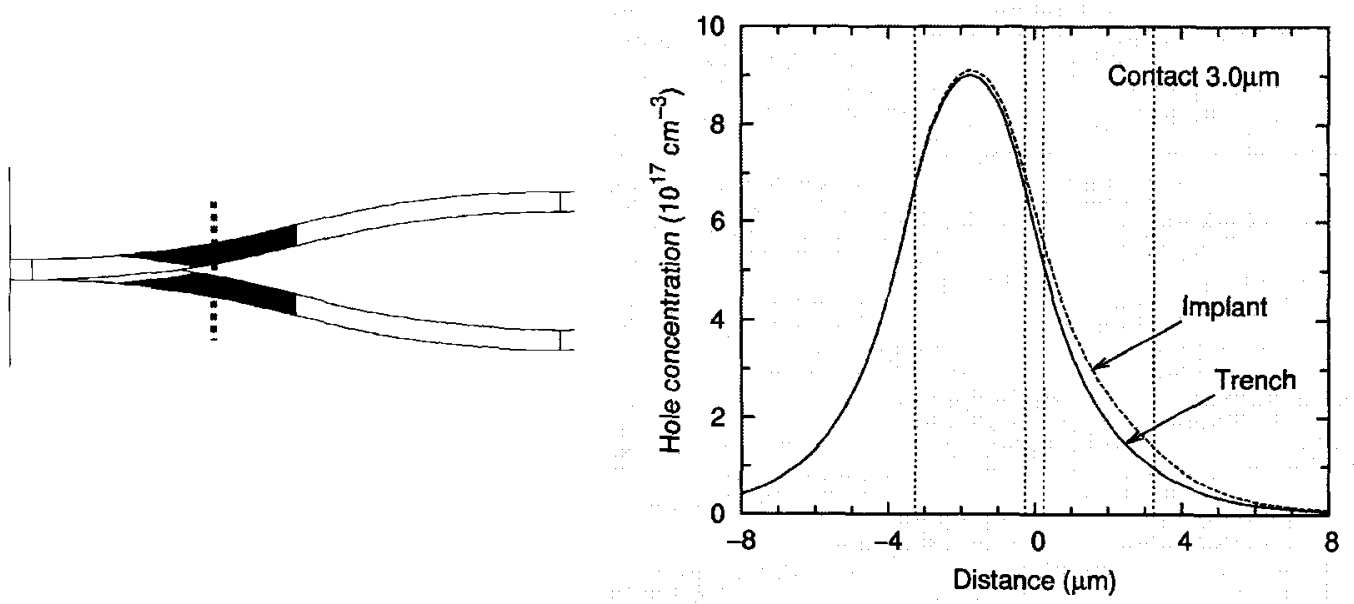

(a)
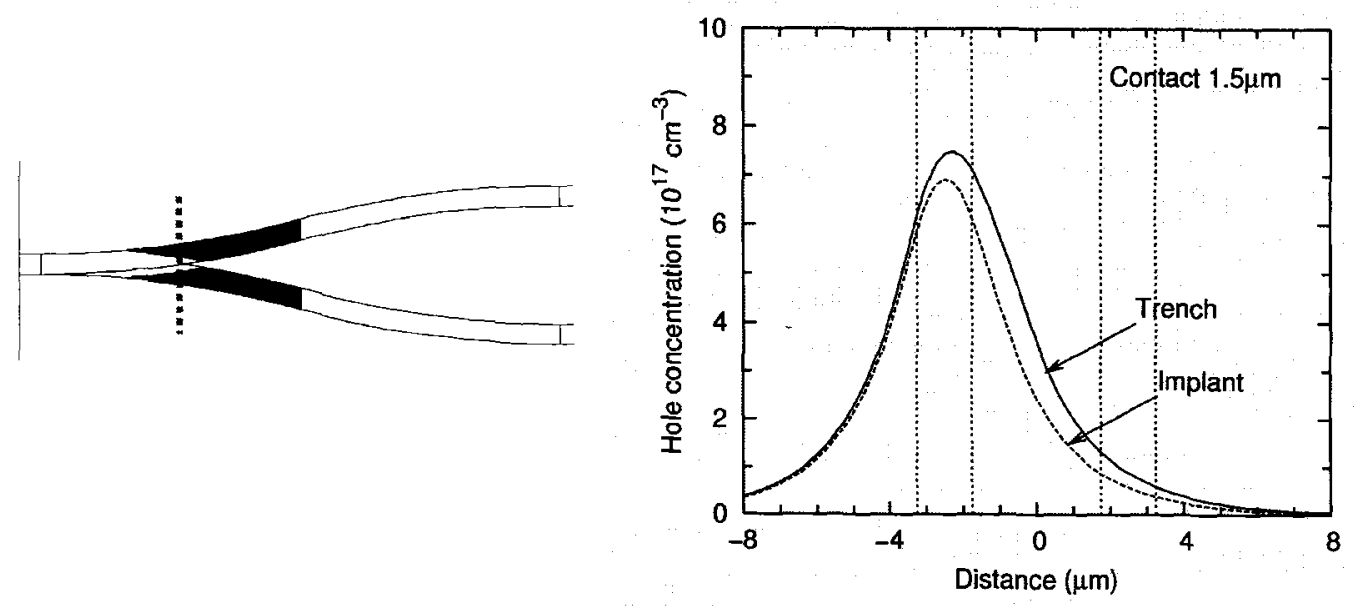

(b)
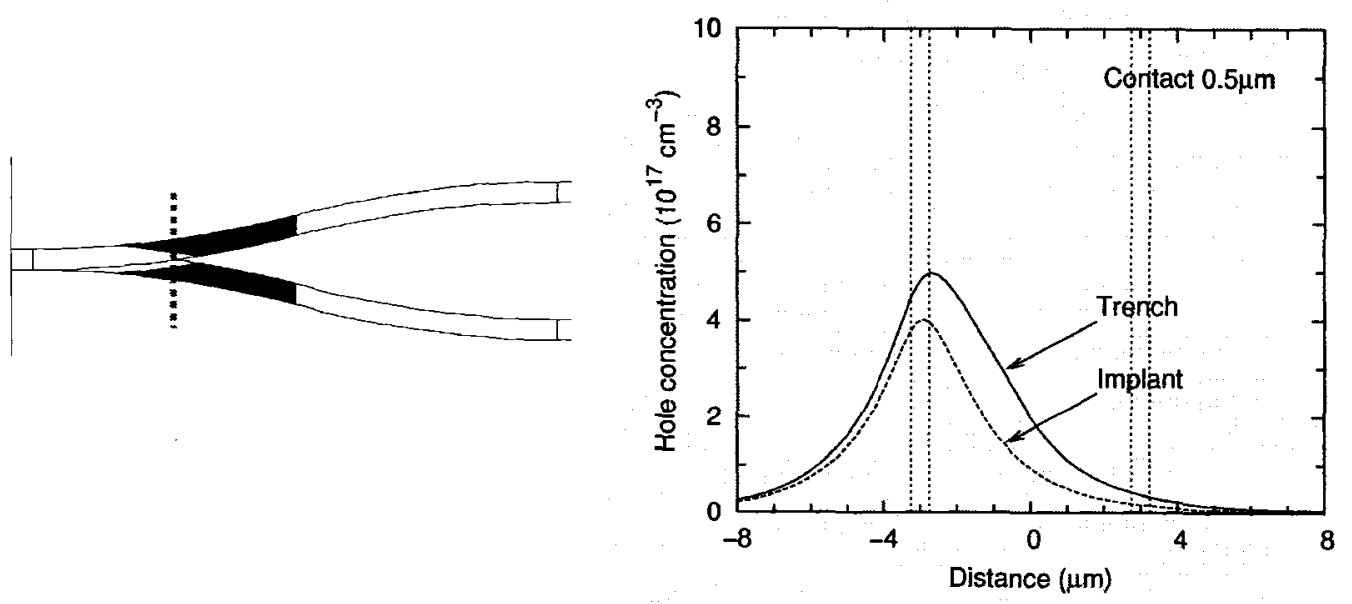

(c)

Figure 5-5 Calculated distribution of holes in the core layer for implant and trench isolation at cross sections where the electrodes are (a) $3 \mu \mathrm{m}$, (b) $1.5 \mu \mathrm{m}$, and (b) $0.5 \mu \mathrm{m}$ wide. The dotted vertical lines show the position of the overlying electrode. 
There was a concern that because the trench was very narrow $(0.5 \mu \mathrm{m})$, it might not be possible to etch down to the etch stop layer, and residual conductive p-doped material would allow a leakage current between the two electrodes. However simulation results (Figure 5-6) show that leaving an unetched layer of up to $300 \mathrm{~nm}$ would not significantly affect hole concentration. Also, SEM images show that the trench is etched within $20 \mathrm{~nm}$ of the etch stop so incomplete etching is not of concern.

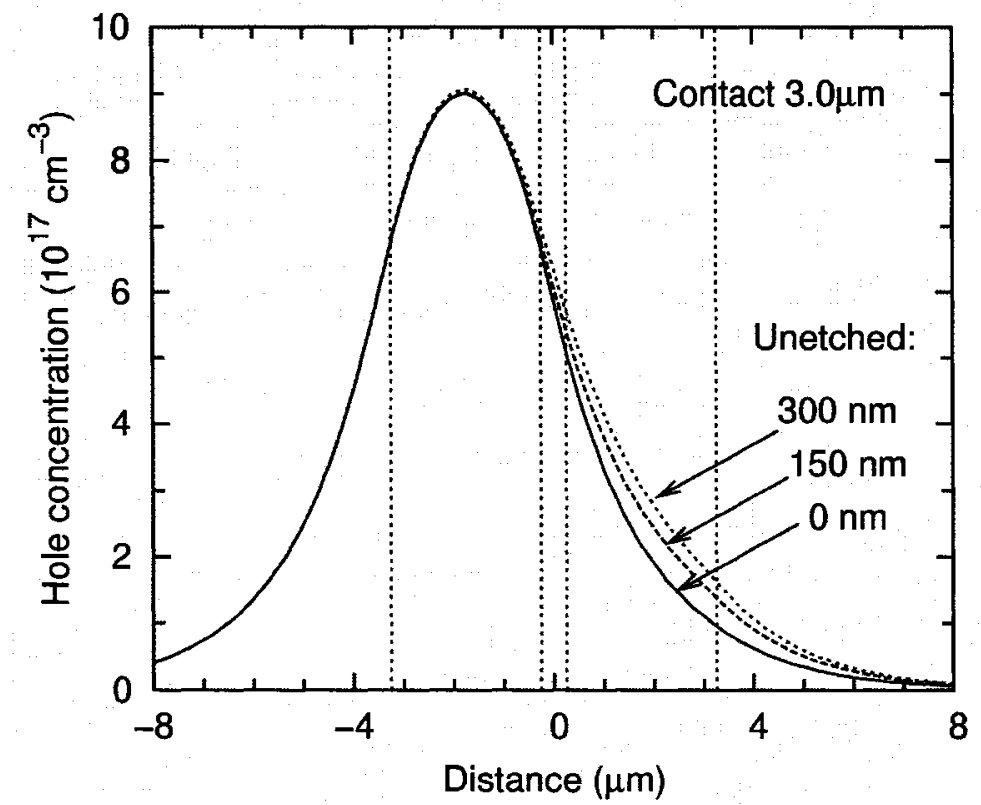

Figure 5-6 Drift diffusion modeling of incomplete etching of trench.

While replacing the implant with the trench was shown to successfully isolate the two contacts of the DOS, the effect of isolation technique on the active performance of the switch is more difficult to model. It is desirable that the carrier distribution follow the electrode shape; however the electrical current of holes flowing down from the contact through the $p$-layer can flow laterally and distort that shape. With an ion implant, this current spreading is almost negligible because the implant drastically reduces the conductivity outside the region under the contacts, thereby suppressing the lateral flow of current (Figure 5-7 (left)). This is not the case with the isolation trench, as shown in Figure 5-7 (right). Figure 5-5 (b) and (c) show the hole distribution in the core at a cross-section along the length of the electrode where the contact covers only 1.5 and $0.5 \mu \mathrm{m}$ of the ridge. With the ion implant, there is a large lateral shift in the hole distribution which follows the shape of the metal electrodes as it narrows, in compari- 
son with Figure 5-5 (a) where the contact covered the full width of the ridge. This shift in hole distribution position is larger with the implant isolation than the trench isolation, and it more accurately reproduces the shape of the overlying electrode on the waveguide. With the trench isolation, there is no high resistivity region immediately adjacent to the electrode, thus the current density spreads out within the ridge into the region beside the contact and up to the trench. Thus, use of the trench for isolation will result in greater current spreading that will cause a larger distortion of the shape of the induced index modulation region.

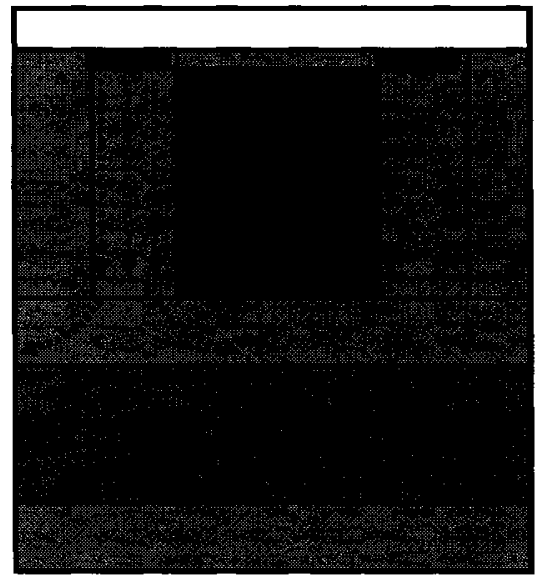

(a)

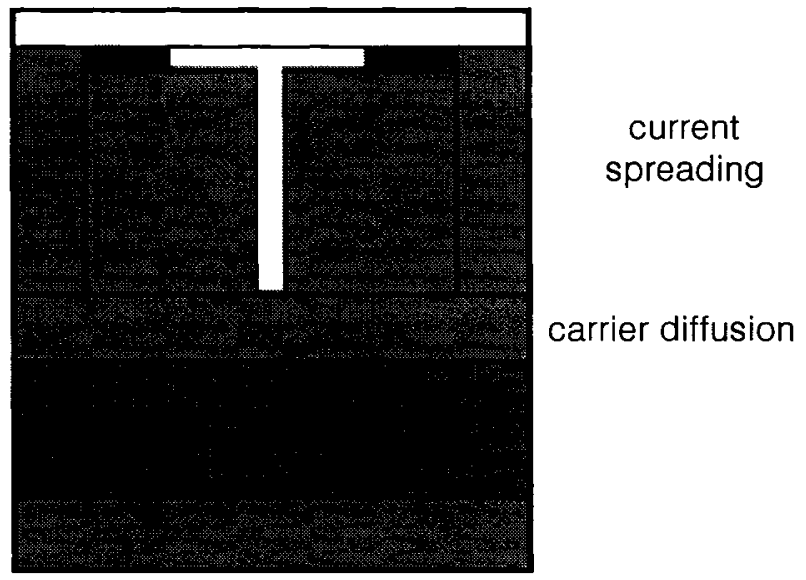

(b)

Figure 5-7 Illustration of current spreading and carrier diffusion in structures with (a) implant isolation and (b) trench isolation.

Due to a concern that the presence of the trench affects the optical performance of the switch, both structures are simulated with two dimensional beam propagation method (BPM) calculations using the effective index method (Figure 5-8). Some scattering can be observed at the trench, however the power difference at the output between the two designs is less than $0.5 \mathrm{~dB}$ for a switch with a branching angle of $0.9^{\circ}$. Thus, the presence of the trench does not significantly increase excess loss. This is a result of the waveguide mode being tightly confined vertically to the InGaAsP core layer, thereby preventing the trench, which does not reach the core layer, from greatly influencing the mode. 


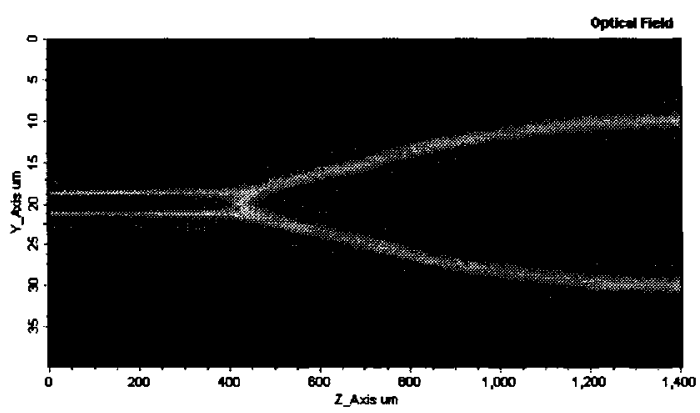

(a)

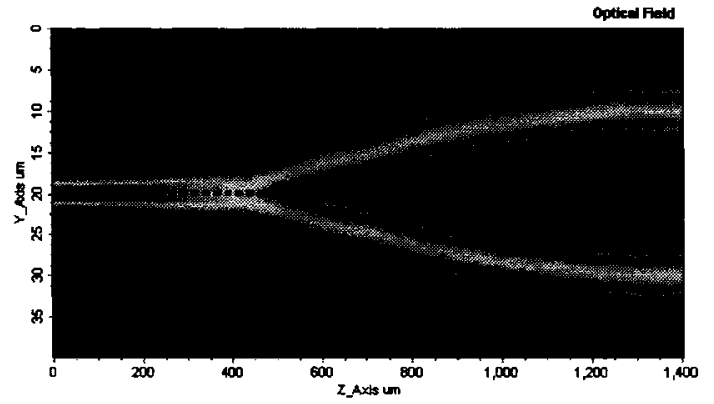

(b)

Figure 5-8 BPM results comparing $0.9^{\circ}$ branching angle DOS without (a) and with (b) $0.5 \mu \mathrm{m}$ wide isolation trench at junction.

\subsubsection{Experimental Results}

The current-voltage (IV) characteristics measured between the two contacts at different stages of fabrication are presented in Figure 5-9 (a). The top curve was taken after removal of the InGaAs contact layer before any isolation process. The bottom two curves show the IV characteristics of the final devices: after InGaAs removal for the devices with trenches, and after ion implantation for the devices without trenches. The ion implantation achieved about three orders of magnitude increase in the resistance between contacts when compared to just after the InGaAs contact layer was removed. The trench process also achieved a large increase in the resistance between contacts when compared to samples without the trench. However, the implantation process produced slightly more isolation than the trench scheme, possibly due to $\mathrm{O}^{+}$ going past the etch-stop layer and perhaps into the core. Additionally, the trench may have increased leakage current by introducing stresses at the bottom corners and defects at the unpassivated sidewalls. 


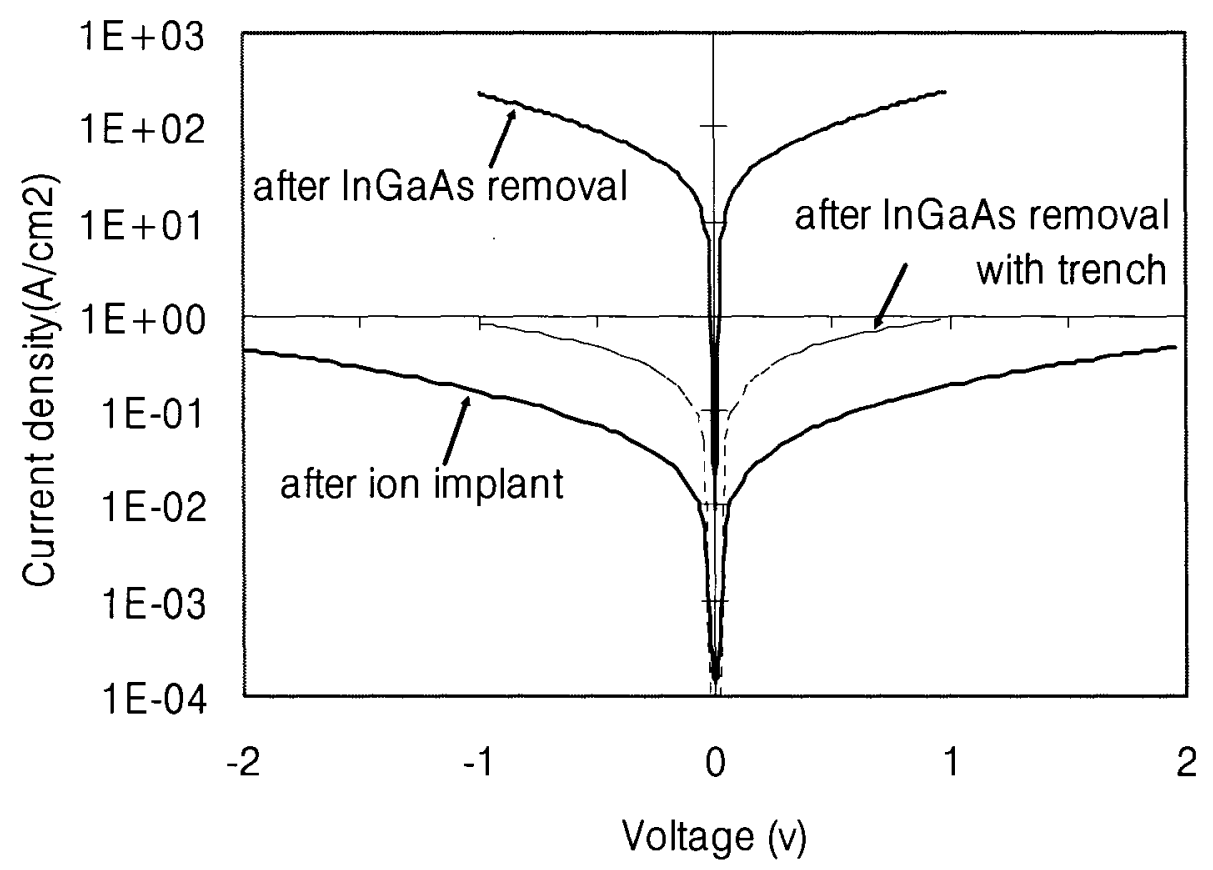

Figure 5-9 Experimental measurements of current versus voltage between the contacts.

Optical characterization of the DOS comprised coupling light from a broadband erbium doped fiber amplifier source $(\lambda=1530-1560 \mathrm{~nm})$ into the devices and collecting the output with a tapered fiber connected to an InGaAs photodetector. Figure 5-10 shows the measured switching curves versus applied current for devices with ion-implant and trench isolation. The DOS had branching angles of $\sim 1.2^{\circ}$ and were characterized with TE polarized light.

In general, the devices with the isolation trench do switch at approximately the same current but do not achieve as large a contrast as the ion implantation processed devices. The reason for this is likely the additional current spreading that occurs in the devices with the isolation trench. A contrast ratio of better than $20 \mathrm{~dB}$ is measured for the otherwise identical DOS with implant isolation, while those with trench isolation achieved less than $10 \mathrm{~dB}$ contrast. Both methods of electrical isolation demonstrated switching times of about $5 \mathrm{~ns}$, determined by carrier diffusion and recombination, and about $15 \mathrm{~mA}$ switching current. All RW-DOS devices discussed in the remainder of this thesis used $\mathrm{O}^{+}$-implant isolation. 


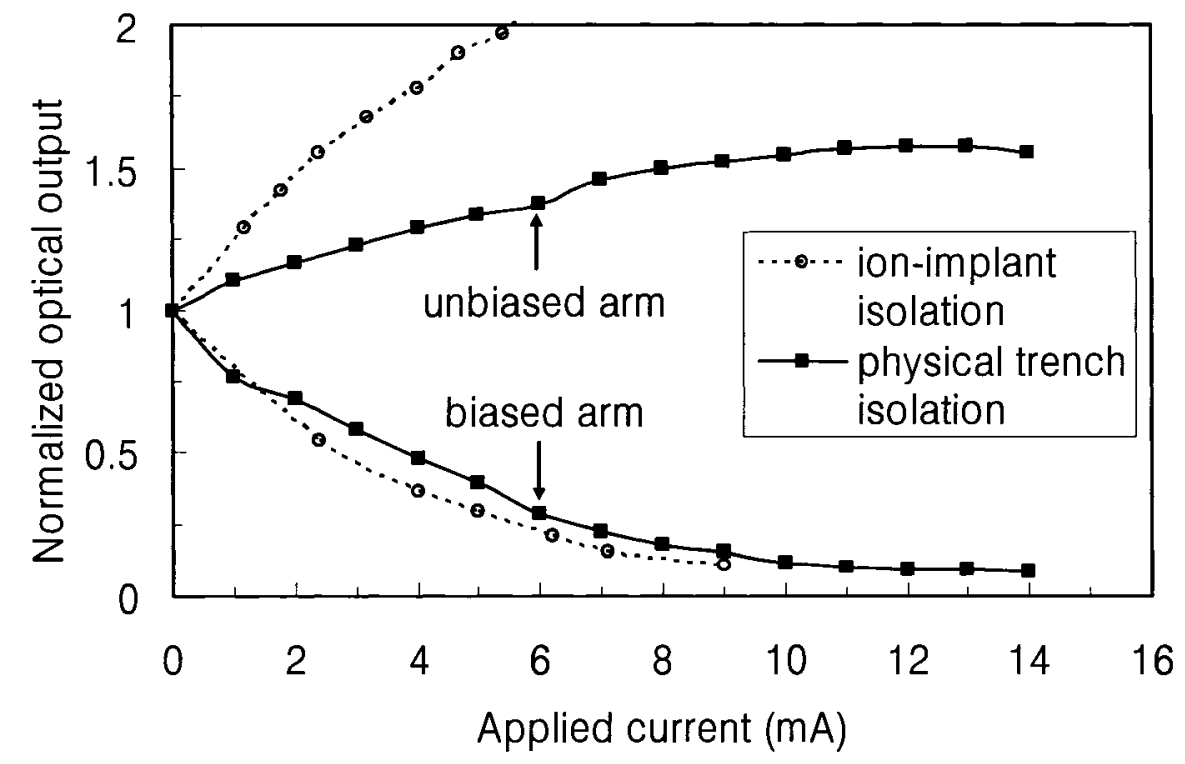

Figure 5-10 Experimental measurements of optical power in biased and unbiased arms of DOS devices with branching angle of $1.2^{\circ}$.

\subsection{Carrier Injection}

DOS devices were also characterized with broadband light centered near $1550 \mathrm{~nm}$ from an $\mathrm{Er}^{+}$-doped ASE source. Figure 5-11 shows the measured output power from the unbiased and biased arms of a DOS upon application of a 100 ns current pulse of 12 $\mathrm{mA}$. Plots are shown for devices with three different core alloys (a) $\mathrm{Q}=1.4 \mu \mathrm{m}$, (b) 1.3 $\mu \mathrm{m}$, and (c) $1.2 \mu \mathrm{m}$, and for both polarizations. The data are normalized to the output power appearing in each arm when no bias is applied.

As predicted by $\mathrm{BPM}$ modeling, for the $\mathrm{Q}=1.4 \mu \mathrm{m}$ alloy the power in the unbiased arm is more than double the power at zero bias because of the decrease in loss at the Y-junction. The measured switching contrast ratio is largest for the $\mathrm{Q}=1.4 \mu \mathrm{m}$ material. This confirms the predictions of both the optical modeling in Figure 3-14(a) and the carrier induced index change calculations presented in Figure 4-5. Specifically, at a $12 \mathrm{~mA}$ bias current the contrast ratio is better than $25 \mathrm{~dB}$ for $\mathrm{TE}$ and $12 \mathrm{~dB}$ for TM polarizations. As discussed previously, this polarization dependence arises because the effective index step between the ridge mode and the slab mode of the etched region 
adjacent to the ridge is smaller for TE polarized light than for TM polarized light. Contrast ratios for the larger bandgap $\mathrm{Q}=1.3$ and $1.2 \mu \mathrm{m}$ materials are smaller $(14$ and $7 \mathrm{~dB}$ respectively), although transmission power is higher.
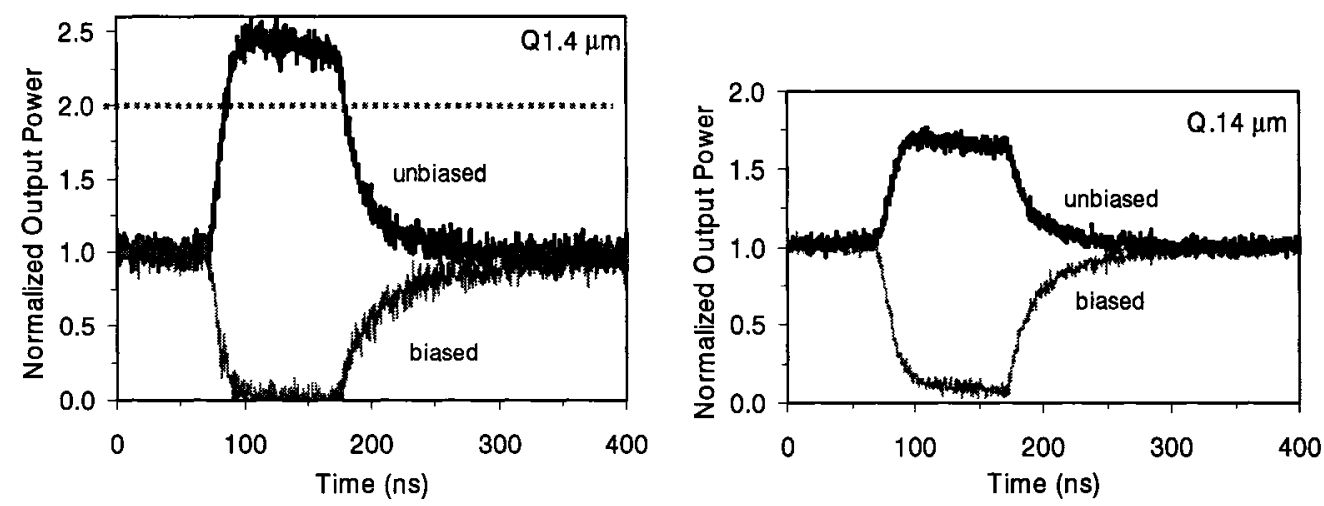

(a)
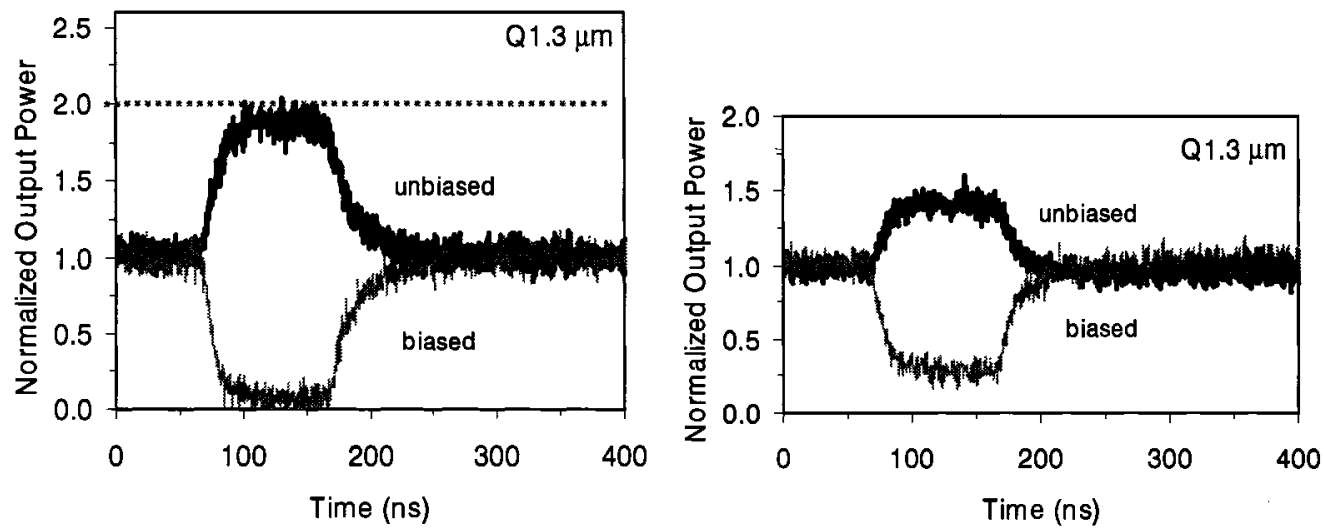

(b)
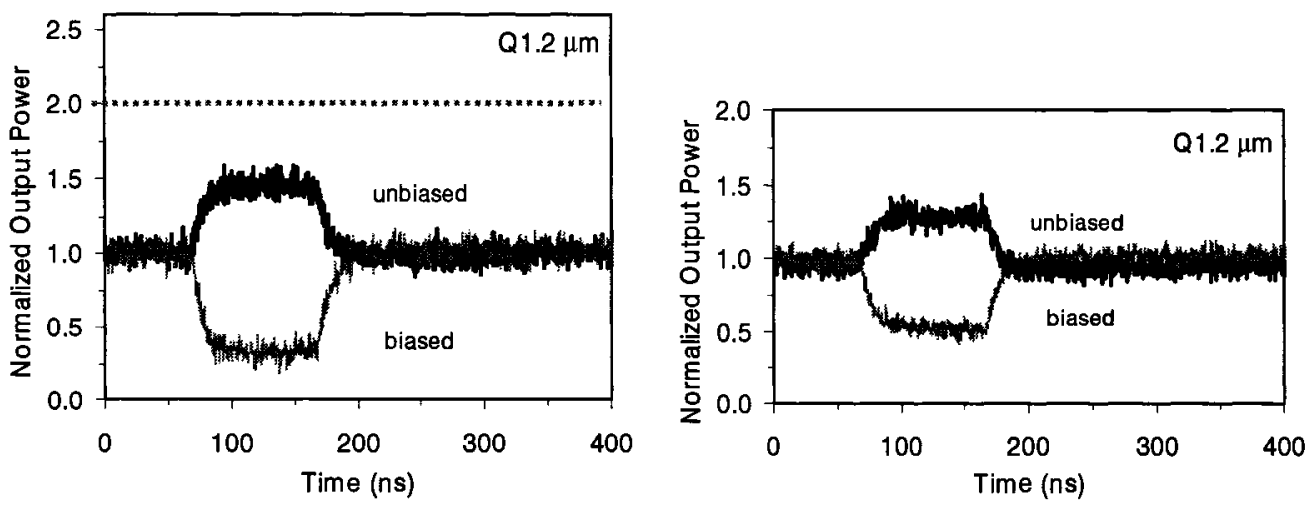

(c)

Figure 5-11 Experimental data from RW-DOS devices on MOCVD wafers with core of alloy (a) Q1.4 $\mu \mathrm{m}$, (b) Q1.3 $\mu \mathrm{m}$, and (c) Q1.2 $\mu \mathrm{m}$. TE polarization is 
shown in the left column and, and TM in the right column. The bias current pulse is $100 \mathrm{~ns}$ long with an amplitude of $12 \mathrm{~mA}$.

Wafers grown by different methods and under different conditions have different densities of recombination centers. These determine the SRH recombination time, which as mentioned previously dominates the carrier lifetime $\tau$ of our devices. Thus we expect to be able to observe differences in device response speeds attributable to varying material defect density. Indeed, the measured $1 / e$ fall times of the DOS devices were $10 \mathrm{~ns}, 15 \mathrm{~ns}$, and $25 \mathrm{~ns}$ for the $\mathrm{Q}=1.2 \mu \mathrm{m}, 1.3 \mu \mathrm{m}$, and $1.4 \mu \mathrm{m}$ alloys respectively. Through a deconvolution with the measured $7 \mathrm{~ns}$ system response time, the corresponding material response times are determined to be 3,7 , and $17 \mathrm{~ns}$. This trend is similar to previously reported values of lifetime for $\mathrm{Q}=1.2 \mu \mathrm{m}, 1.3 \mu \mathrm{m}$, and 1.4 $\mu \mathrm{m}$ bandgap InGaAsP of $11 \mathrm{~ns}, 20 \mathrm{~ns}$, and $32 \mathrm{~ns}$ respectively [85].

The differences in defect density should also have an effect on the index change per applied current density $d n / d j$ slope as well as the current and power required for switching. To test these hypotheses, a second set of RW-DOS and MZ devices were fabricated on wafers grown by molecular beam epitaxy (MBE) for comparison.

Figure 5-12 shows the optical output power from the output arms of DOS devices on MOCVD and MBE grown wafers of $\mathrm{Q}=1.4 \mu \mathrm{m}$ InGaAsP upon application of $100 \mathrm{~ns}$ current pulses of $5 \mathrm{~mA}$. The $20-80 \%$ rise time of DOS devices on the MOCVD wafers is $20 \mathrm{~ns}$, whereas the rise time of devices on the MBE wafers is about $40 \mathrm{~ns}$. The longer switching time suggests a longer SRH lifetime due to a lower defect density of the MBE material. 


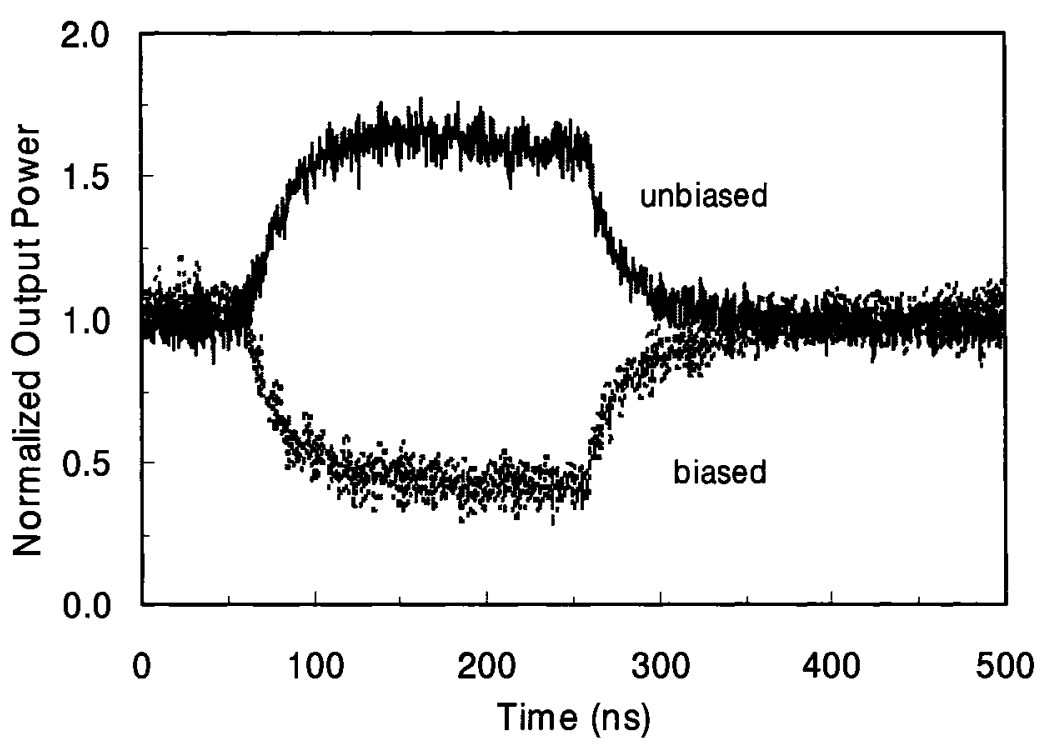

(a)

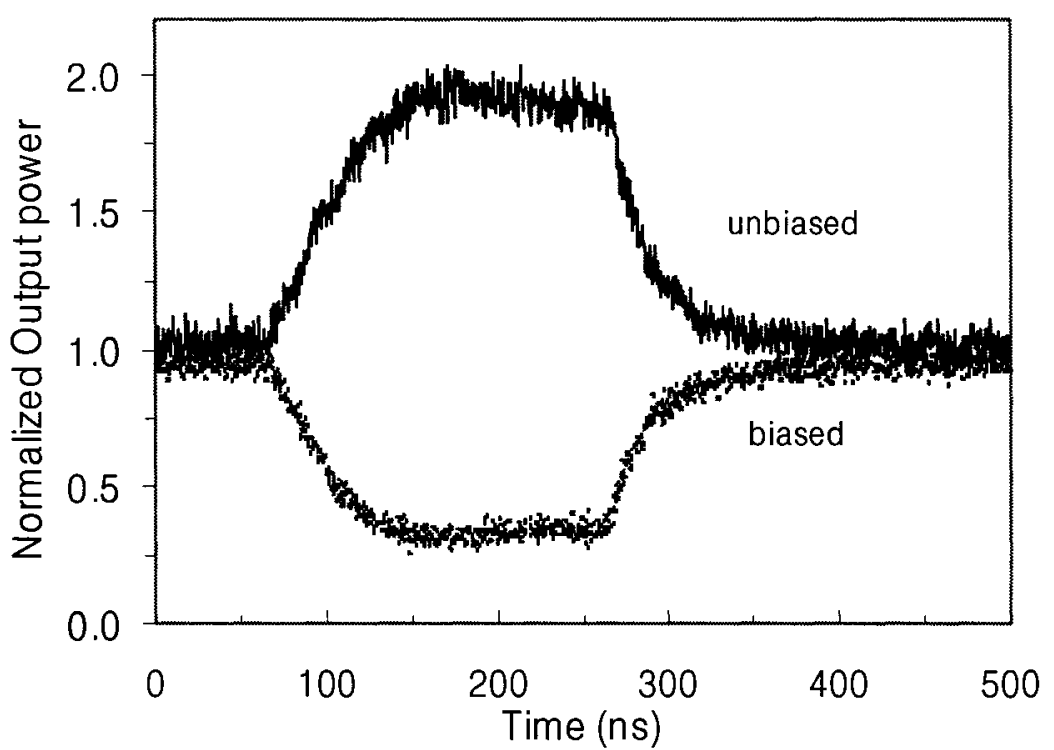

(b)

Figure 5-12 Experimental data from digital optical switch (DOS) devices on (a) MOCVD and (b) MBE wafers switched with $200 \mathrm{~ns}$ pulses of $5 \mathrm{~mA}$.

The noise in the optical output signal may be due to electrical noise from the optical detector, jitter in the sampling oscilloscope and movement of the input and output fibers causing fluctuations in optical coupling. The difference in noise level between the two arms of the same switch is due to the difference in absolute power coupled out 
of the biased and unbiased arms. The relative signal-to-noise ratios are consistent for the same device.

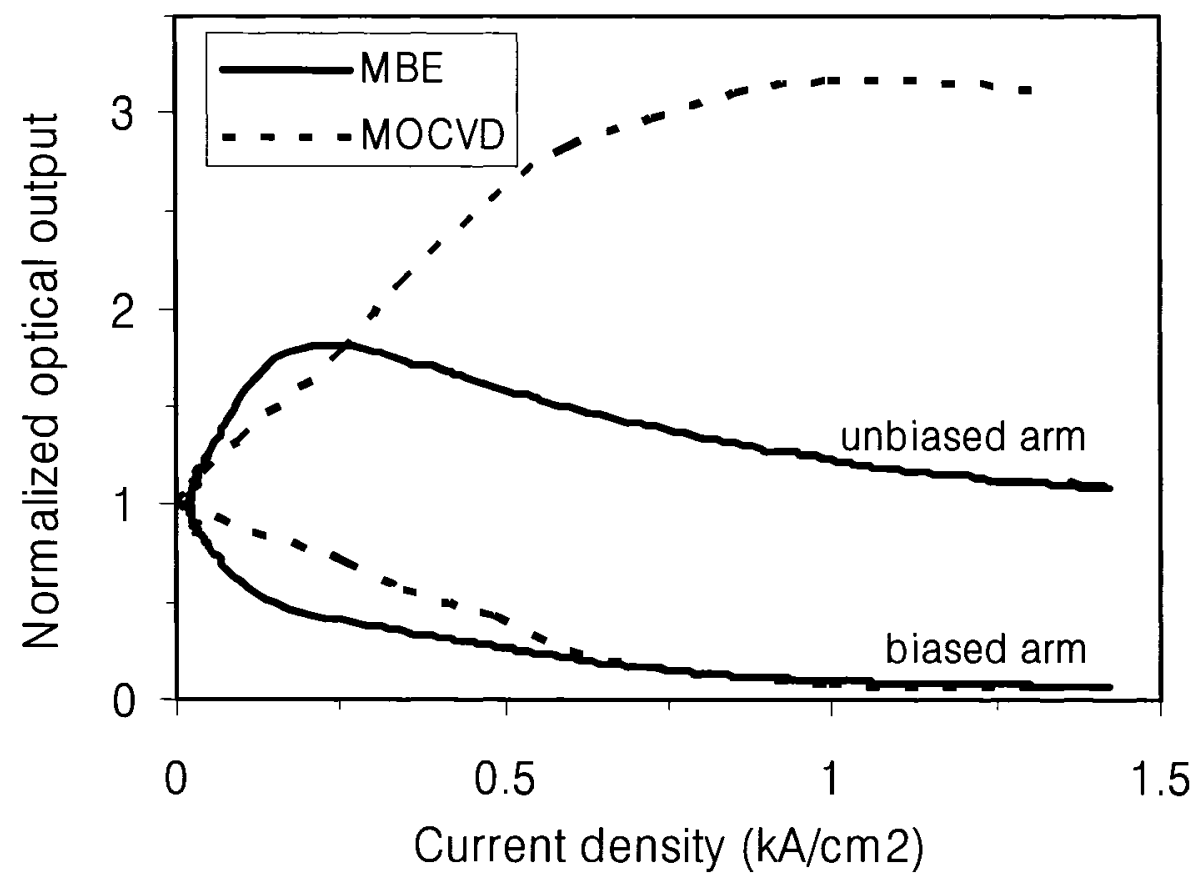

Figure 5-13 Experimental data from digital optical switch (DOS) devices on MBE and MOCVD wafers.

The optical output power as a function of applied current density for DOS devices on an MOCVD wafer is shown in Figure 5-13. Power in the unbiased arm reaches a peak at an applied current density of about $1 \mathrm{kA} / \mathrm{cm}^{2}$, corresponding to the point at which the induced index change $\Delta \mathrm{n}$ is effectively equal to effective index step $\delta n_{\text {eff }}$ and the effective waveguide configuration is a single bent waveguide as in Figure 3-2 (b). Also shown in Figure 5-13 are results for a matching DOS device on an MBE wafer. The $0.25 \mathrm{kA} / \mathrm{cm}^{2}$ required to achieve maximum output power in the unbiased arm is about four times less than the $1 \mathrm{kA} / \mathrm{cm}^{2}$ required for the DOS on MOCVD to attain peak power in the biased arm. This smaller current density is consistent with the MBE material having a longer carrier lifetime, and the ratio is similar to that of $d n / d j$ mentioned above. The longer lifetime allows a larger carrier density to accumulate, inducing a larger index change, for a given applied current density. However, the MBE switch achieves a smaller contrast ratio due perhaps to the broader refractive index profile that results from greater carrier diffusion. This was shown in Figure 4-6, where 
the carrier distributions at different current densities are presented to compare the index profiles for different carrier lifetimes at the carrier concentration corresponding to the switching peaks.

Thus we see evidence from switching speed, index change versus current density, and switching current that the MBE material has a three to four times longer lifetime than the MOCVD material. In operating the DOS devices by current injection, we face a tradeoff between switching speed, switching current or power, and potentially switching contrast. Nevertheless, the required driving current for the MOCVD devices is an order of magnitude lower than the $200 \mathrm{~mA}$ in our first reported devices [86]. The reduced current is a result of optimization of the InGaAsP alloy as well as a reduction in material defect density resulting in longer carrier lifetimes. Previous versions of the RW-DOS devices showed large thermal heating effects [87], but through material optimization, carrier injection effects became dominant.

Finally there are implementation differences between MOCVD and MBE growth. MOCVD, having a higher growth rate and being more cost efficient than MBE, is more commonly used in industry. MBE is operated in vacuum and so should achieve lower defect concentrations. However, since MOCVD is dominant in InP based optoelectronic epitaxy, the process is well-refined.

As mentioned previously, a comparison of switching performance could not be easily performed on the MOCVD grown wafers due to the difference in waveguide confinement between the three core alloys. Accordingly, the switching performance is now compared for the MBE grown wafers in which the layer structure has been designed so that waveguide confinement is consistent between the three alloys.

Figure 5-14 is a plot of the measured switch output power in each arm and contrast between the two arms of the DOS devices fabricated on MBE grown wafers. The $\mathrm{Q}=1.4 \mu \mathrm{m}$ device has a larger contrast than the $\mathrm{Q}=1.3 \mu \mathrm{m}$ device which has a larger contrast for the $\mathrm{Q}=1.2 \mu \mathrm{m}$ devices for the same applied current density. In comparison with MOCVD results, these are not attributable to differences in waveguide confinement and so confirm that improved switching performance may be achieved through the use of a smaller bandgap core alloy or material with longer carrier lifetime. 
The TM polarization shows a smaller contrast than the TE, as is expected from the larger waveguide effective index contrast. The contrast of the $\mathrm{Q}=1.2 \mu \mathrm{m}$ devices continues to increase to about $12 \mathrm{~dB}$ at $6 \mathrm{kA} / \mathrm{cm}^{2}$.

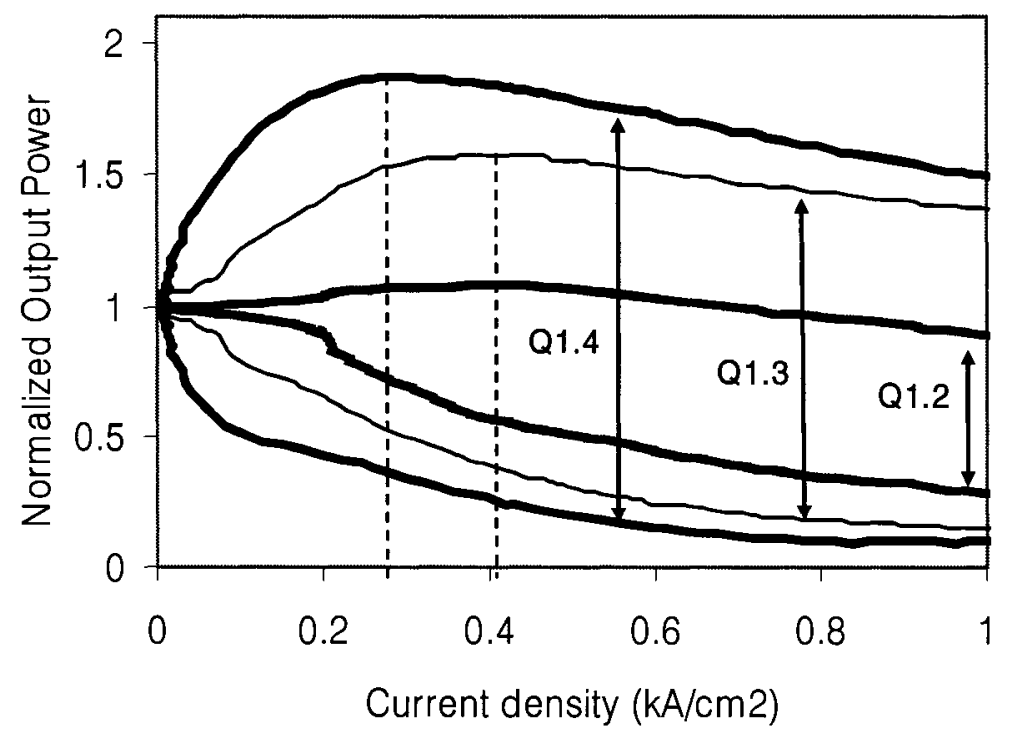

(a)

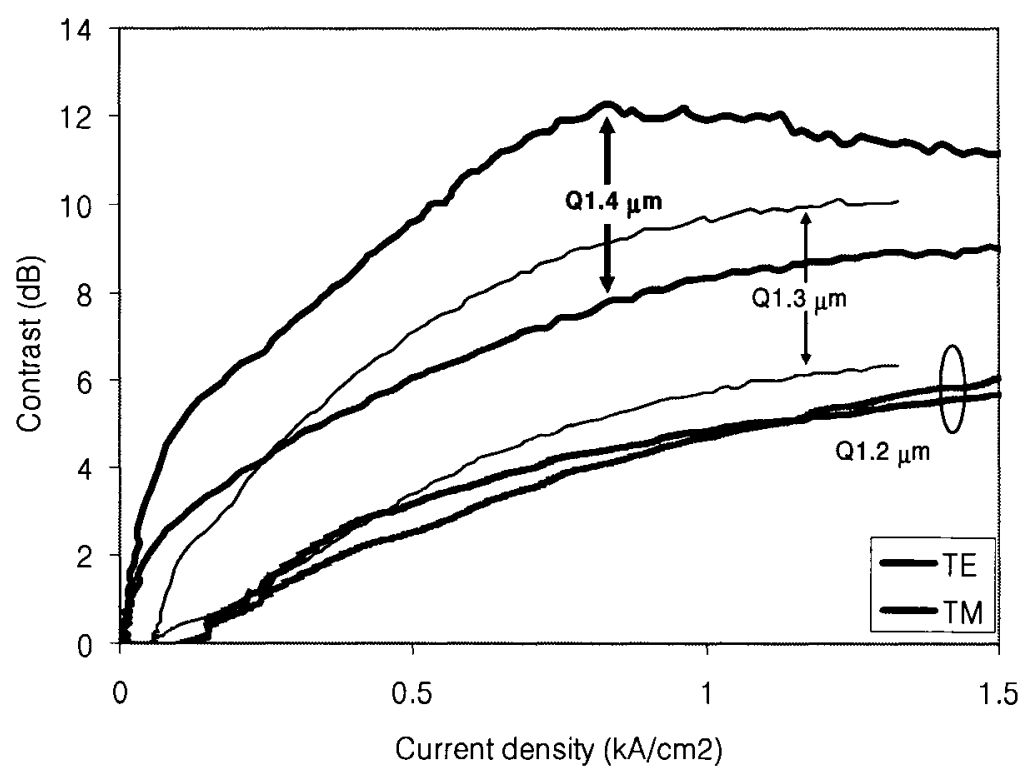

Figure 5-14 Experimental data comparing the (a) optical output in TE polarization and (b) contrast of $1.35^{\circ} \mathrm{DOS}$ devices with core alloys of $\mathrm{Q}=1.2 \mu \mathrm{m}, 1.3$ $\mu \mathrm{m}$, and $1.4 \mu \mathrm{m}$. 
A comparison of the switching performance with MZI measurements of index change helps to understand the performance of the switch. Figure 5-15 is a plot of the measured index change versus applied current density for each of the three MBE grown wafers. The effective index difference $\mathrm{n}_{\mathrm{TE}}$ between the ridge waveguide and slab region is $\sim 2.5 \times 10^{-3}$ and is indicated by the horizontal dashed line.

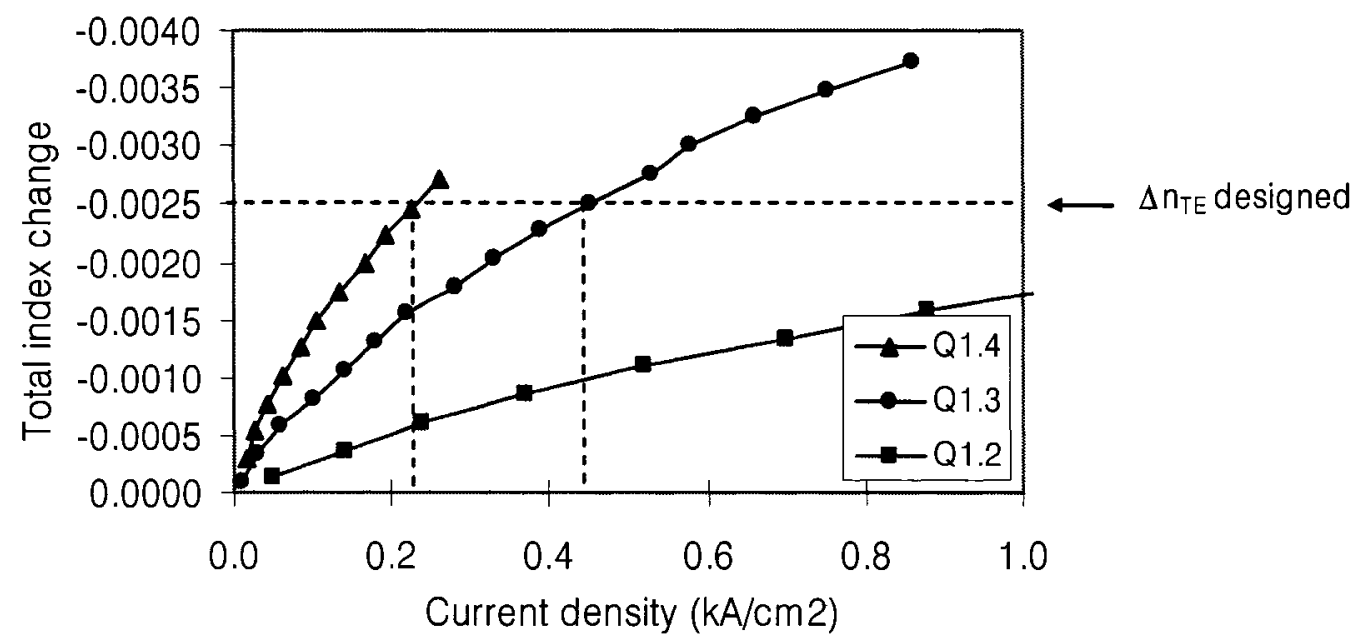

Figure 5-15 Experimental data in TE polarization of index change versus current density as calculated from MZ fringes.

The maximum output power from the unbiased arm of the $\mathrm{Q}=1.4 \mu \mathrm{m}$ device occurs at a current density of $\sim 0.25 \mathrm{kA} / \mathrm{cm}^{2}$ as indicated by a dashed line in Figure $5-14(\mathrm{a})$. This current density corresponds to an index change equal to $\delta n_{T E}$ as determined from the MZ interferometer measurements shown in Figure 5-15. This agreement in current density at these two points substantiates the model that when the refractive index of the biased arm is reduced to the effective index of the adjacent slab region, the effective waveguide configuration of the DOS device is an ideal single bent waveguide which transmits maximum power to the unbiased output arm. Likewise, the current density at maximum output for the $\mathrm{Q}=1.3 \mu \mathrm{m}$ device is about $0.4 \mathrm{kA} / \mathrm{cm}^{2}$, which is similar to the $0.45 \mathrm{kA} / \mathrm{cm}^{2}$ expected from the $\mathrm{MZ}$ measurements. For the $\mathrm{Q}=1.2 \mu \mathrm{m}$ device, there is very little power switched into the unbiased arm. Additionally, the MZ results for this quarternary show that the index change does not reach $\Delta \mathrm{n}_{\mathrm{TE}}$ until a current density of more than $2 \mathrm{kA} / \mathrm{cm}^{2}$. The increased absorption losses and carrier diffusion at this large current density may explain the poor switching performance using this quarternary. 


\subsection{Overdriving}

The response time of the DOS devices on the MOCVD wafers is approximately $20 \mathrm{~ns}$. However, since the switch response is digital rather than oscillatory, overdriving can be used to increase the leading edge rise time of the switched optical pulse [88]. Nevertheless, the fall time of the pulse will still be limited by carrier lifetime and diffusion. Therefore, to improve overall switching speed it is necessary to apply a reverse bias when the electrode is in the off state. The reverse bias rapidly sweeps out the carriers from the waveguide core after the electrical switching pulse turns off. For the MOCVD devices, by overdriving, rise and fall times have been reduced to the $7 \mathrm{~ns}$ resolution of our experimental setup.

The increase in response speed due to overdriving is illustrated in Figure 5-16 for RWDOS devices with $\mathrm{Q}=1.4 \mu \mathrm{m}$ alloy core and an applied current pulses of $50 \mathrm{~ns}$ duration. Figure 5-16 shows the power in the two output arms for $12 \mathrm{~mA}$ current pulses and zero reverse bias, and for overdriving with $22 \mathrm{~mA}$ current pulses and $-1.5 \mathrm{~V}$ reverse bias. The switching times of these DOS devices on MOCVD wafers are reduced from $\sim 20 \mathrm{~ns}$ down to less than $10 \mathrm{~ns}$ on both the leading and trailing edges. The switching times of the DOS devices on the MBE wafers are reduced from $\sim 40 \mathrm{~ns}$ down to less than $10 \mathrm{~ns}$ by overdriving from $5 \mathrm{~mA}$ to $9 \mathrm{~mA}$.

Another consequence of overdriving is increased index modulation, absorption and scattering of the biased arm due to the higher carrier densities generated by overdriving, which all serve to enhance contrast. In this way by overdriving to $22 \mathrm{~mA}, \mathrm{TM}$ polarization also achieves $25 \mathrm{~dB}$ contrast ratio, resulting in polarization independent switching contrast. Thus the polarization dependence of the waveguides results only in a polarization dependent loss that is due to the difference in power coupled into the unbiased arm. 


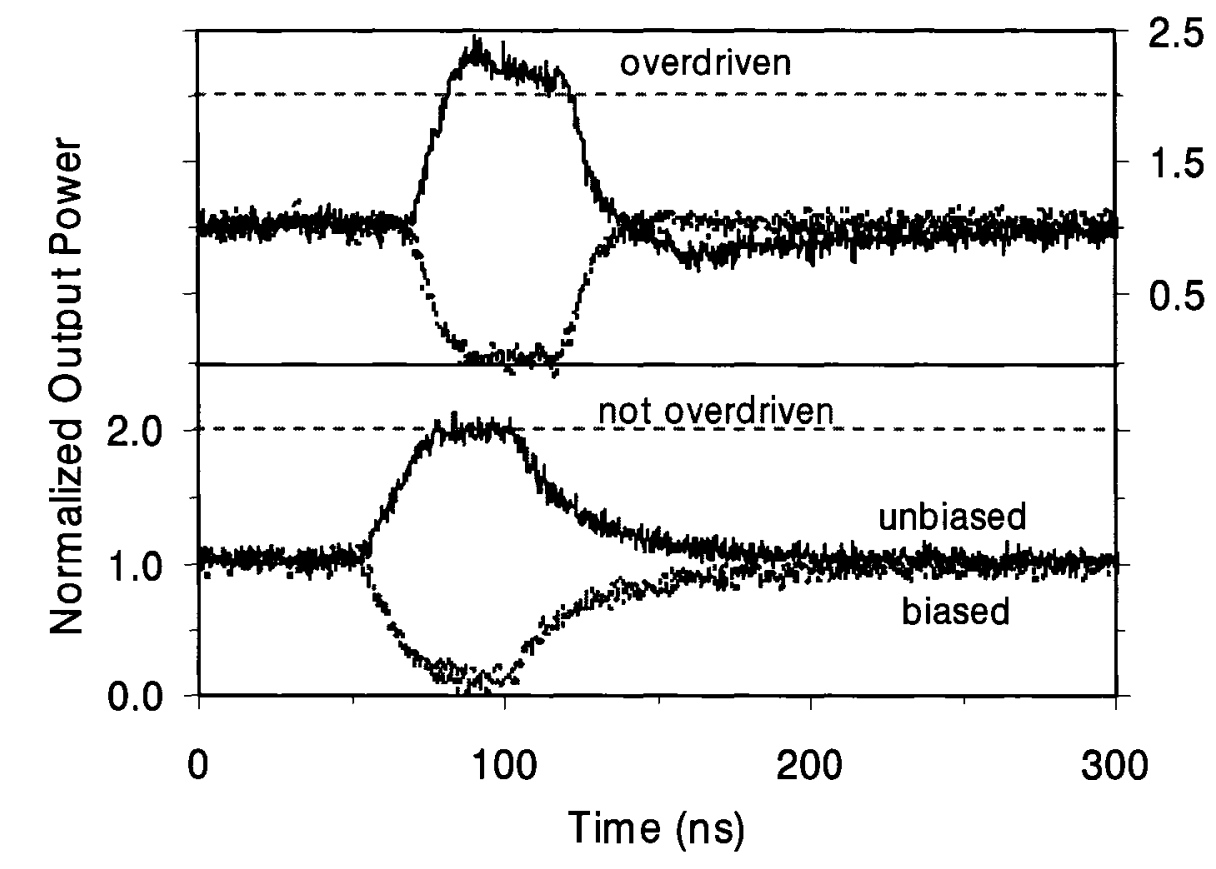

(a)

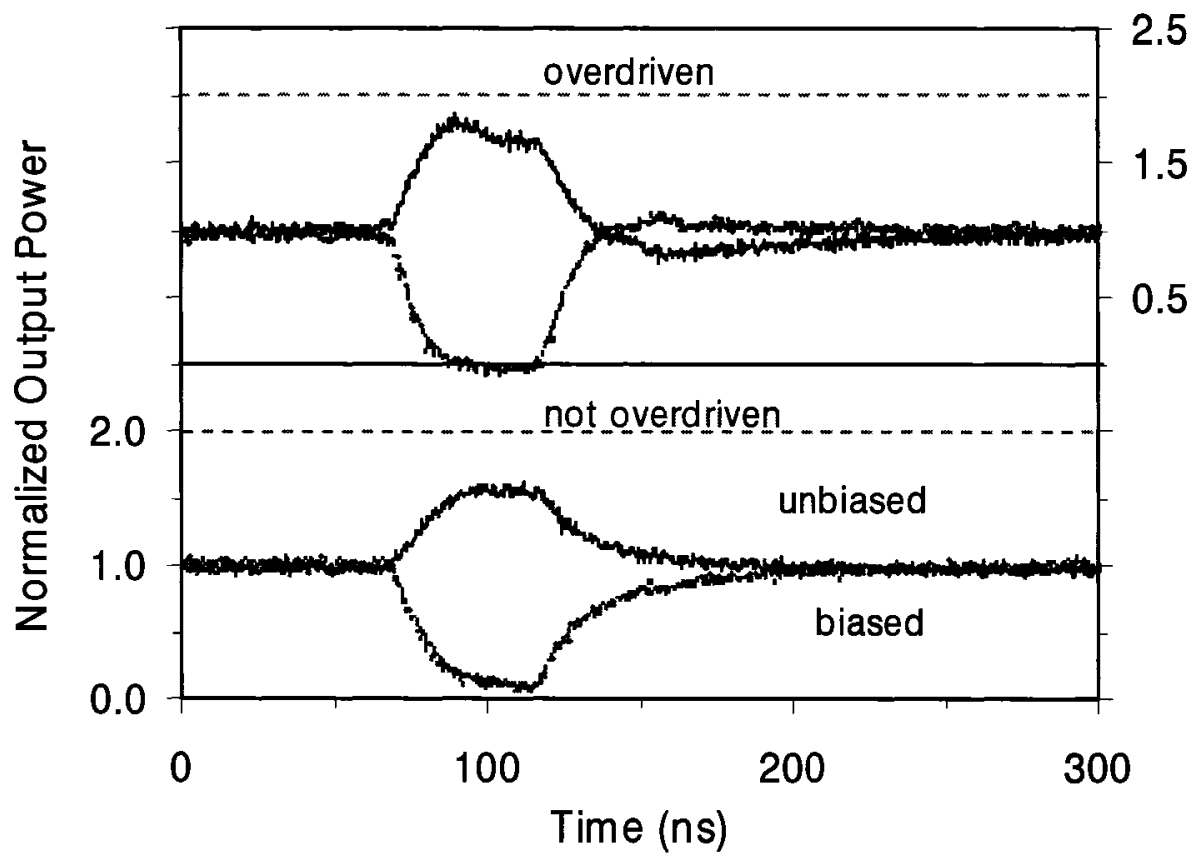

(b)

Figure 5-16 Experimental output from switched DOS comparing no overdrive $(12 \mathrm{~mA})$ and overdriving $(22 \mathrm{~mA}$ and reverse bias of $-1.5 \mathrm{~V})$ with a $50 \mathrm{~ns}$ pulse for (a) TE and (b) TM polarizations. The core alloy is InGaAsP with $\mathrm{Q}=1.4$ $\mu \mathrm{m}$. 
However, overdriving causes the falling edge of the unbiased arm to suffer an overshoot before returning to its initial level. The depth of the overshoot is dependent upon the current pulse width and height, and the recovery time is on the order of $100 \mathrm{~ns}$. This suggests a thermal gradient effect in which a steep temperature gradient is established very quickly and decays over a few hundred nanoseconds [87]. This temperature gradient results in a local positive refractive index gradient across the Y-junction.

\subsubsection{Discussion}

The choice of material bandgap determines device performance. Because wavelengths closer to the band edge undergo larger refractive index changes per applied current density, a DOS with a smaller bandgap core alloy requires less power for switching. Furthermore, the material lifetimes of undoped InGaAsP peak around the $\mathrm{Q}=1.4 \mu \mathrm{m}$ bandgap alloy [85], resulting in the largest carrier concentration per applied current for this quaternary. The $\lambda=1550 \mathrm{~nm}$ signal may suffer higher losses though, through increased absorption.

Better material quality also increases carrier lifetime, allowing larger carrier concentrations to accumulate for the same applied current. This reduces the current density, and therefore power, required for switching. However, longer lifetimes also result in longer switching times, given that response speeds of devices actuated by current injection are determined by carrier recombination rates. In addition, the increased carrier lifetime allows greater carrier diffusion, thereby reducing index gradients and decreasing switching performance. In our RW-DOS switch design, establishing a steplike index modulation profile is very important, because reconfiguring the light path relies on a strong local index gradient.

The effect of carrier diffusion on index profile was not included in the optical simulations used in this thesis. A more detailed analysis of a few selected cases indicates that including more realistic modal field and carrier distribution profiles can shift the experimentally determined index modulation by up to a factor of two. This may be in part responsible for the discrepancy between experimental and theoretical values of index change in Figure 4-9. However a full two dimensional simulation of both optical 
and dynamic electrical behaviour of the switch waveguide structure is well beyond the scope of this work.

\subsection{Quantum Well Implementation}

The RW-DOS devices with quantum well cores were tested under forward bias. Figure 5-17 shows the oscilloscope traces of biased and unbiased output for the $0.78^{\circ}$ DOS with $5 \mu \mathrm{m}$ waveguide width on wafer RQWa with an applied current pulse of about $25 \mathrm{~mA}$. A contrast ratio of better than $10 \mathrm{~dB}$ is achieved. Operation at the single $1600 \mathrm{~nm}$ wavelength introduces Fabry-Perot interference fringes at the leading and trailing edges, which prevents accurate measurement of the switch rise and fall times. Connectorization of the switch to fiber pigtails will prevent such interference fringes.

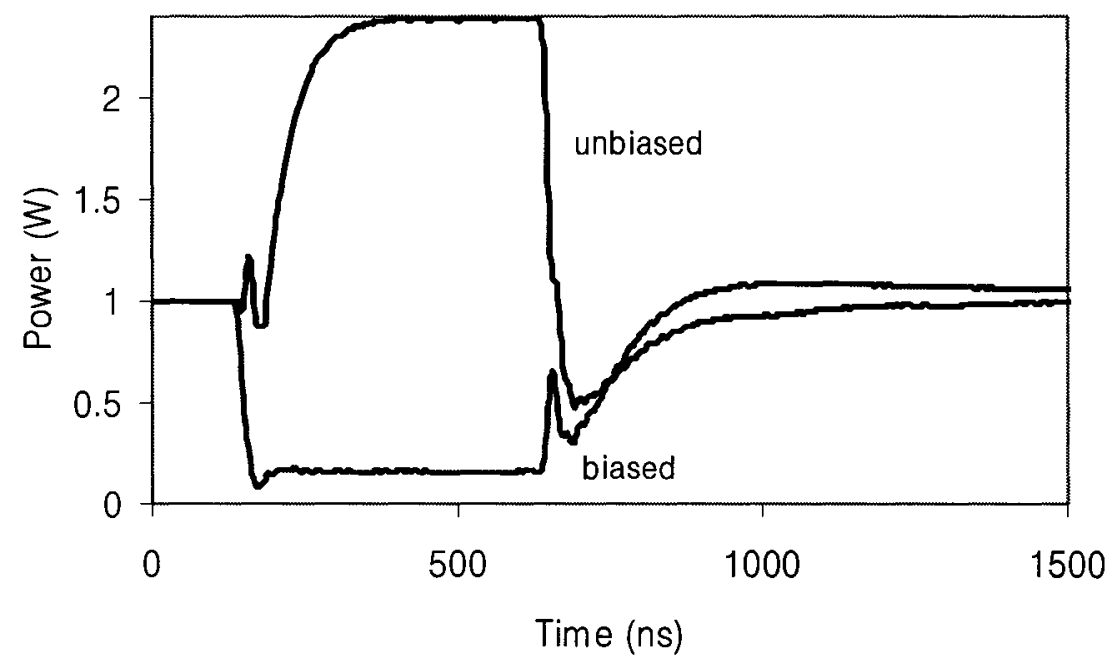

Figure 5-17 Oscilloscope trace of $0.78^{\circ}$ DOS on wafer RQWa in TE polarization.

Under reverse bias, the RW-DOS devices with quantum well cores were expected to achieve shorter switching times because of the faster electro-optical response. However, the optical performance of the switch changes under such operation because the electro-optical effect increases refractive index whereas carrier-injection decreases it. For the RW-DOS device, a positive induced index change under an electrode refracts 
light into the biased arm, while a negative induced index change guides light into the unbiased arm. In the former case, the refraction of light results in the existence of an optimal branching angle. Furthermore, there is potentially greater leakage into the "off" arm since unlike the case where the biased arm is effectively destroyed by a decrease in refractive index, a positive index change leaves the "off arm" with its original effective index and still capable of guiding. BPM calculations of the fabricated structures and for the case of positive $\Delta \mathrm{n}$ predicts switching for $|\Delta \mathrm{n}|>0.003$, limited to a switching contrast of about $30 \mathrm{~dB}$ as opposed to $\sim 40 \mathrm{~dB}$ for negative index changes.

As mentioned previously, the QW wafers were characterized with an L-band tunable laser because transmission was poor in the C-band. The applied voltage is measured by contacting the electrode with a second probe that is connected to a one megaohm input impedance in the oscilloscope. This minimizes the additional load and prevents it from drawing additional current.

Fabry-Perot fringe measurements on straight waveguides under reverse bias showed that an index change of $\Delta \mathrm{n} \sim 0.0002$ could be achieved with an applied negative voltage of $1 \mathrm{~V}$ for both of the RQW and with $5 \mathrm{~V}$ for the 3SQW. The RQW results are in agreement with previously published results that state an index change of about $1 \times 10^{-4} / \mathrm{V}[65]$.

The measured switching response for the biased and unbiased arms is shown in Figure 5-18. Light from a tunable laser source at $\lambda=1600 \mathrm{~nm}$ is coupled into the input waveguide. The light is directed into the biased arm, consistent with a positive index change expected for the QCSE effect in an InGaAsP RQW structure. As expected of electro-optical actuation, the response time is less than the 7 nanosecond resolution of the experimental system. A switching contrast ratio of better than $5 \mathrm{~dB}$ was achieved.

The index changes achieved at the maximum $-8 \mathrm{~V}$ applied bias induce an index change of only about -0.0016 , which is half that required in simulations to achieve full switching. Additionally, the poor contrast ratio may be a result of loss due to the red shift in the absorption spectrum at the large applied voltages. Maximum contrast for $\mathrm{TE}$ polarization is not reached until a reverse bias voltage of about $-7 \mathrm{~V}$ is applied, as 
seen in Figure 5-18 above. But even at a bias of $-2 \mathrm{~V}$ the spectrum is sufficiently shifted that signals at $1600 \mathrm{~nm}$ see a decrease in transmission, as seen Figure 4-12. Thus the contrast may be limited by the onset of absorption. Use of a longer wavelength, further from the bandedge, does not improve performance. At $1530 \mathrm{~nm}$, refractive index modifications are smaller by about 70 percent, as is switching contrast $(\sim 4 \mathrm{~dB})$.

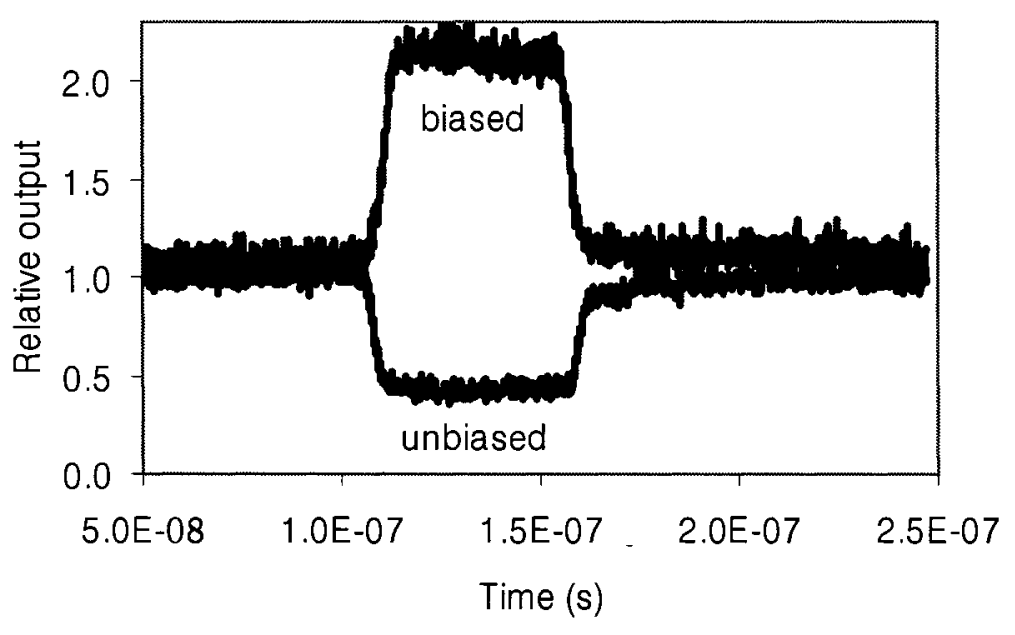

(a)

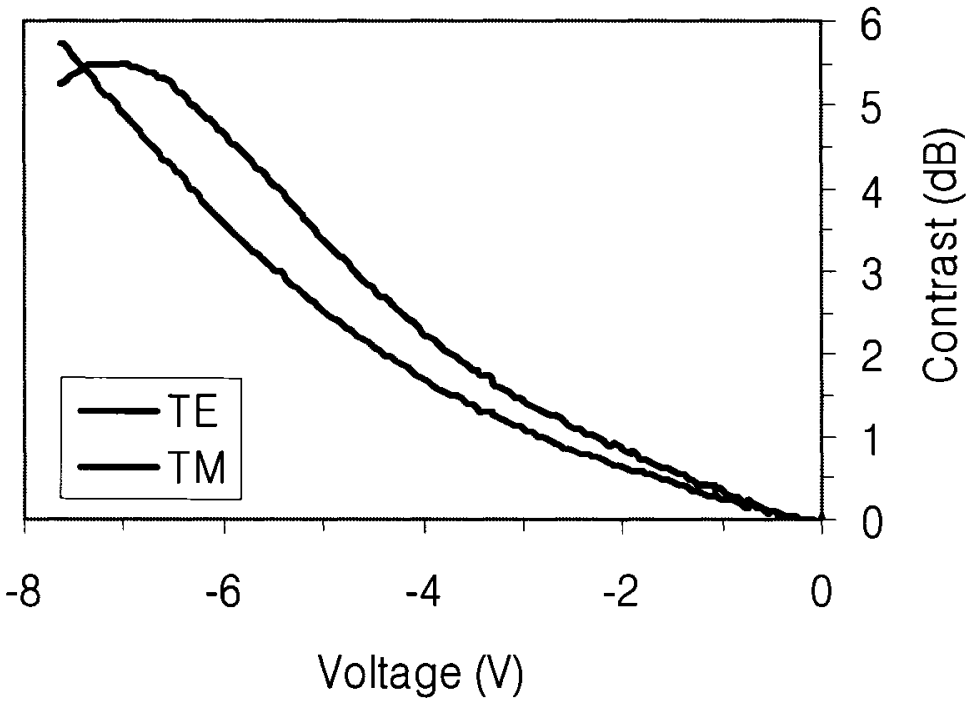

(b)

Figure 5-18 Experimental results from the RQWa wafer showing (a) optical output power versus time and (b) switching contrast versus applied reverse bias. 


\subsection{Summary}

Electrical isolation of contacts in a Y-junction DOS was studied both theoretically and experimentally. BPM calculations establish that the excess optical loss introduced by the trench is less than $0.5 \mathrm{~dB}$. Two sets of switches were demonstrated. In the first set, isolation by ion implantation resulted in measured switching contrasts of better than $20 \mathrm{~dB}$. In the second set, physical isolation was achieved with the trench, resulting in switching contrasts of less than $10 \mathrm{~dB}$. The reduced contrast is explained by the increased current spreading that occurs in the absence of implantation isolation, and underscores the importance of the shape of the index modulation in the operation of this switch design. In this DOS, establishing an accurate index modulation shape is dependent upon the limitations of lithography during fabrication, the extent of carrier diffusion as the carriers travel from the electrodes down to the core, and material parameters such as carrier mobility. Stress induced index modulation and leakage current may also play a part in the reduced contrast observed with the physical trench isolated DOS. Most importantly, electrical and experimental results establish that $\mathrm{O}^{+}$ implantation and trench isolation are effective in isolating electrodes with sub-micron spacing, increasing the gap resistivity by approximately three orders of magnitude.

Through characterization of carrier injection in $\mathrm{InGaAsP} / \mathrm{InP}$ waveguide devices, we have demonstrated performance optimization of a DOS switch with reconfigurable output waveguide arms. Switching contrast ratios of better than $25 \mathrm{~dB}$ in both polarizations have been demonstrated in our carrier injection based devices. We have also reduced switching current by an order of magnitude through optimization of wafer and device design. Further reduction in switching current, at the expense of switching speed, is possible through use of material with lower defect density. This tradeoff between current and speed is partially averted by overdriving the current pulses to enhance switching speed. In this way we have demonstrated switching times of less than $10 \mathrm{~ns}$, limited by our experimental setup. As well, electro-optical performance of the RW-DOS was demonstrated to reduce switching speed to below the response speed of the experimental setup. 
The performance of the optimized RW-DOS is sufficient for cascading of the devices, the results which are discussed in the next chapter. 


\section{Chapter 6}

\section{CHARACTERIZATION OF ADDITIONAL CARRIER INJECTION DEVICES}

\subsection{Bend-loss Attenuator}

Optical attenuators are ubiquitous in optical networks. They are used at the source side for pre-emphasis, as well as inline with amplifiers or add/drop multiplexers for channel equalization, and also at the detector side as termination points [89]. In most of these applications the modulation mechanism must be polarization independent and wavelength independent, thus excluding most commonly used electro-optical effect devices. The driving features in the development of attenuators are fast speed, low power consumption, and easy integration with other optical components.

Variable optical attenuators (VOAs) based on micro-electro-mechanical systems (MEMS) are difficult to integrate. Waveguide attenuators can generally be classified into two categories: those based on extinction, and those based on optical path switching. Switch-type structures such as Mach-Zehnder interferometers, directional couplers, and Y-branches [90] are often large because they require sufficient interference lengths to influence light propagation direction. As well, interferometric based devices are wavelength dependent. Extinction-based attenuators are generally straight or bent waveguides, and the optical signal is attenuated through absorption [91] or radiation loss due to reduced confinement [92]. Attenuators based on extinction are 
generally more compact than those based on switching and can make use of existing connection waveguides in a planar lightwave circuit (PLC).

Straight or bent waveguide attenuators have been proposed and successfully commercialized in polymer materials $[48,50]$ with actuation by the thermo-optical effect. However, polymers are sensitive to heat, and optical power, and are often birefringent as well. These issues, along with uncertain long-term stability have delayed the wide acceptance of polymer waveguide devices. Finally, response times for thermo-optical actuation are long, on the order of milliseconds [52].

The ideal material system would allow waveguide attenuators to be integrated into PLCs and even incorporated into existing connection waveguides to minimize device footprint. Fabrication on a III-V semiconductor is conducive to integration with many other optical components [93]. Potential actuation mechanisms in semiconductors include carrier injection, electroabsorption [91], and semiconductor optical amplifier (SOA) based modulation [94]. A preference for polarization and wavelength independence make electro-optical effect and SOA devices unattractive. Carrier injection is much faster than the thermo-optical effect and also wavelength independent and polarization independent. A straight waveguide modulator has been implemented in silicon using carrier injection to suppress the refractive index and modulate by "waveguide-vanishing" [92]. In polymers, several bent waveguide thermo-optical designs have been proposed that enhance modulation by adding bend loss [52, 54]. We propose that the introduction of a simple bend in a waveguide carrier injection modulator implemented in semiconductor will similarly enhance attenuation and reduce actuation power.

\subsubsection{Device design}

The proposed bend loss attenuator consists of a straight waveguide section at the input followed by an s-bend leading to a straight output section. An electrode is placed over part of the s-bend section as shown in Figure 6-1 (a). Note that the electrodes are only in electrical contact with the device where they overlap the waveguide ridges in the s-bend. 
(a)

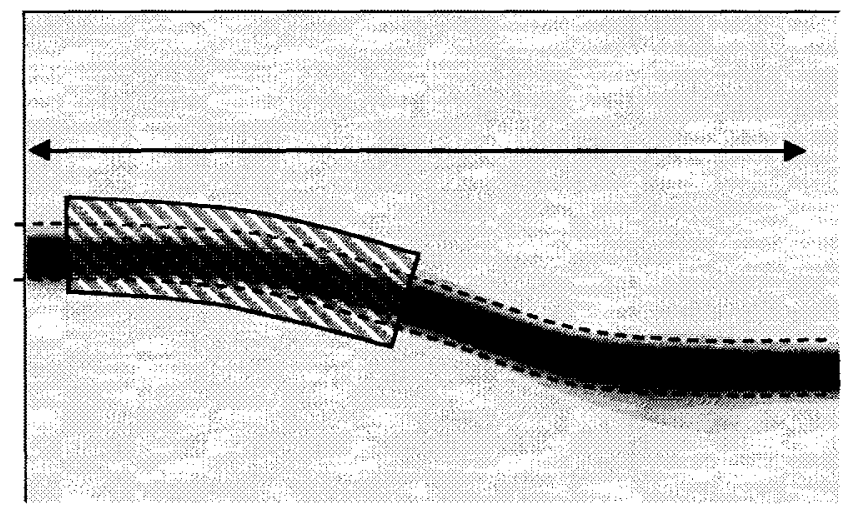

(b)

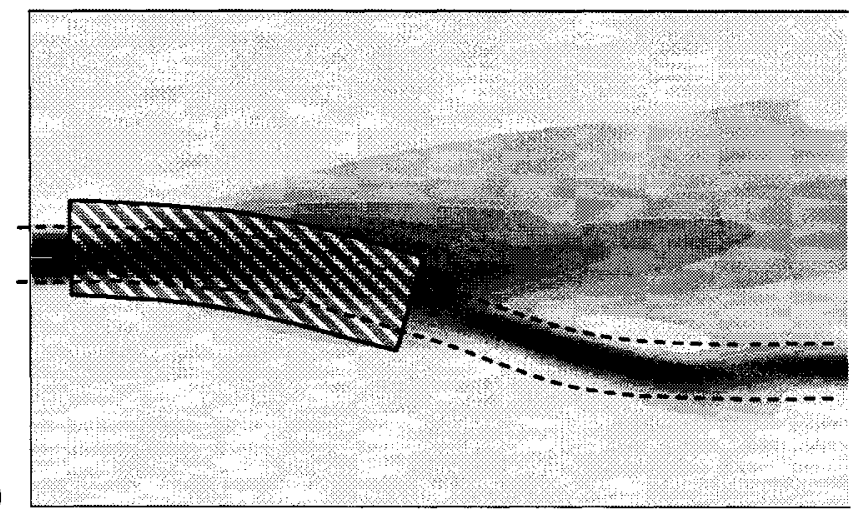

Figure 6-1 BPM simulation results of a bend loss attenuator with input at the left and output at the right showing (a) propagation through the unmodulated device, and (b) propagation with current applied to the electrode causing a negative refractive index change in the waveguide below.

The wafers were grown by metal-organic chemical vapour deposition (MOCVD) with structure described in Section 3.3. Specifically, the layer structure is an

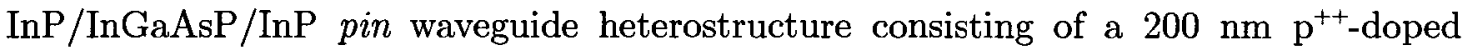
InGaAs top contact layer, a $600 \mathrm{~nm}$ p-doped $\mathrm{InP}$ layer, $300 \mathrm{~nm}$ of undoped $\mathrm{InP}$, the $600 \mathrm{~nm}$ undoped InGaAsP $(\mathrm{Q}=1.4 \mu \mathrm{m})$ waveguide core, another $300 \mathrm{~nm}$ undoped $\mathrm{InP}$ layer, $700 \mathrm{~nm}$ of $\mathrm{n}^{+}$-doped $\mathrm{InP}$, all grown on an $\mathrm{n}^{+}$-doped $\mathrm{InP}$ substrate which acts as a common electrode. The ridge waveguides, defined by e-beam lithograpy, were $5 \mu \mathrm{m}$ wide with a $600 \mathrm{~nm}$ etch depth. The s-bend section had a radius of curvature of 3.2 $\mathrm{cm}$, and the attenuator had an overall length of $2 \mathrm{~mm}$.

With no applied bias, the input power is guided through the bend. Under forward bias, carriers are injected into the core causing a negative index change [59] of about $8.6 \times 10^{-3}$ per $\mathrm{kA} / \mathrm{cm}^{2}[95]$. The reduced refractive index under the electrode results in 
reduced confinement of the waveguide mode, and hence increased loss through the bend as shown in Figure 6-1 (b). When the induced index change $\Delta \mathrm{n}$ is equal to the difference in effective index between the ridge waveguide and the adjacent slab waveguide region, the optical field will no longer see a waveguide and attenuation is at a maximum.

\subsubsection{Simulations}

The bend loss attenuator performance was simulated using two dimensional beam propagation method (BPM) calculations, using the effective index method as described previously with respect to the DOS devices. Attenuation is due to a combination of bend loss (Figure 6-2) and waveguide spoiling (Figure 6-3), which introduces a mode mismatch loss.

Loss due to the bend can be calculated as a succession of mode mismatch losses as the waveguide is displaced laterally through the bend. Figure 6-2 compares the loss for attenuators with bend radii of $30 \mathrm{~mm}, 20 \mathrm{~mm}$, and $10 \mathrm{~mm}$. For a given $\Delta \mathrm{n}$, the attenuation is stronger for a smaller bend radii.

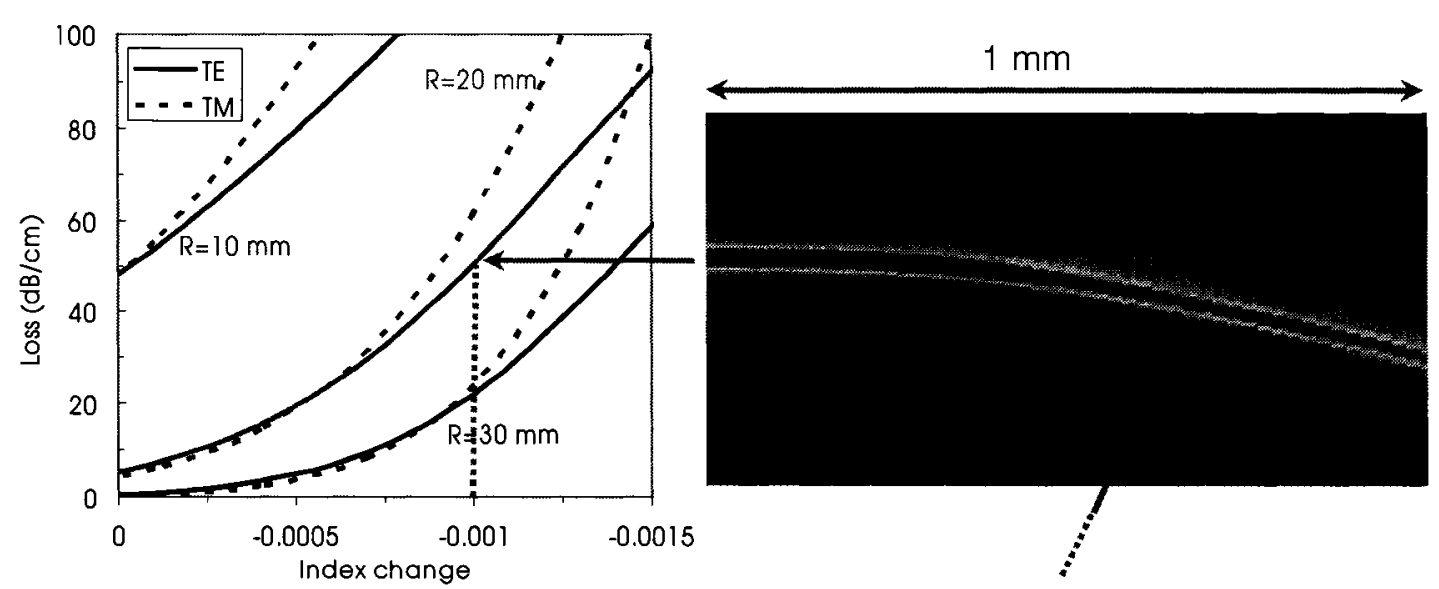

Figure 6-2 BPM results showing loss due to the bend structure for bends of different radii.

Waveguide spoiling is the destruction of the higher effective refractive index waveguiding region beneath the electrode. An example of this is shown in Figure 6-3 where BPM simulations show the effects of carrier injection into an $800 \mu \mathrm{m}$ electrode on straight and bent waveguides. 


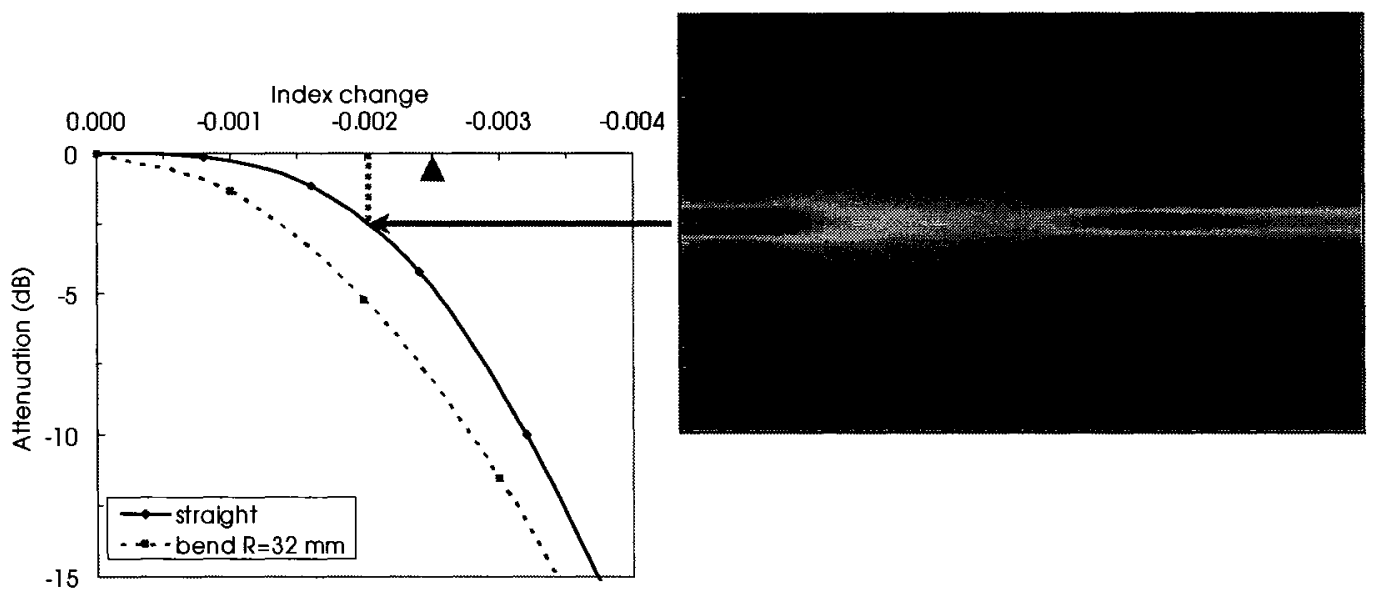

Figure 6-3 BPM results showing loss due to mode mismatch for straight and bent waveguide attenuators.

The sum of these two effects is simulated for a bend-loss attenuator with a chip length of $2 \mathrm{~mm}$ and an s-bend section with a radius of curvature of $32 \mathrm{~mm}$. Figure 6-4 shows the BPM results of output power versus index change for the attenuator with an electrode length of $800 \mu \mathrm{m}$ in comparison with a straight waveguide attenuator of the same overall length and same electrode length. The inclusion of the bend in the device increases the attenuation by up to $5 \mathrm{~dB}$ for the same induced index change. An attenuator with a bend would require about half the current density, and hence half the power, for attenuation to $3 \mathrm{~dB}$ than a straight waveguide.

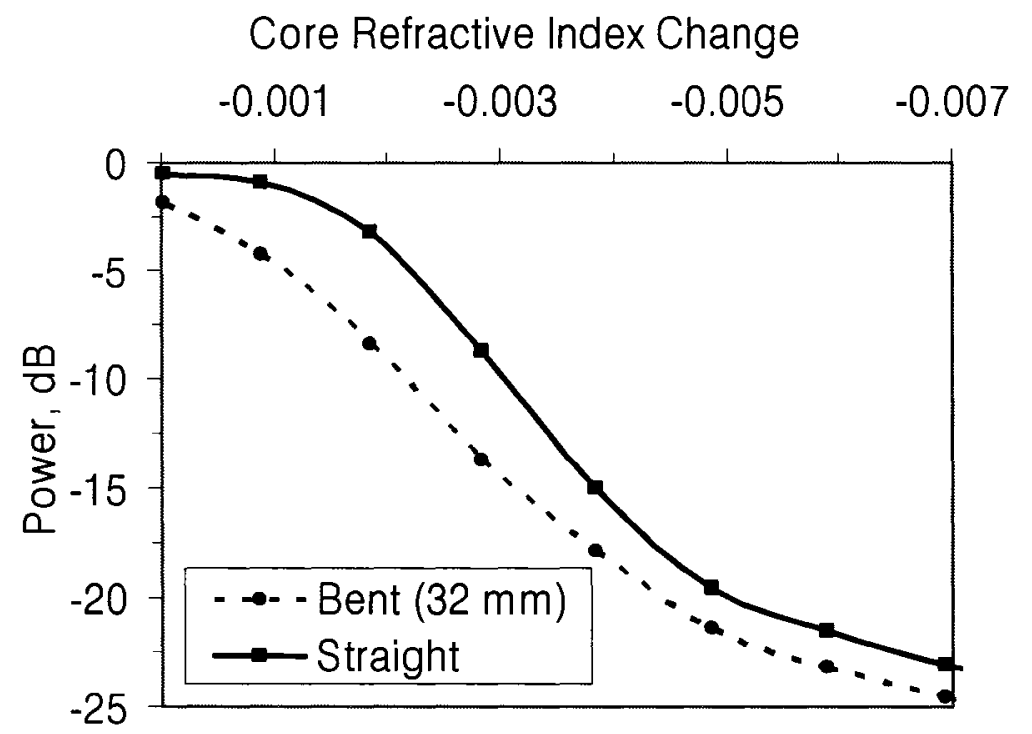

Figure 6-4 BPM results for straight and bent waveguide attenuators. The calculation is for TE polarization and includes carrier induced absorption losses. 


\subsubsection{Experimental results}

Optical characterization of the attenuators comprised coupling light from a broadband erbium doped fiber amplifier source $(\lambda=1530-1560 \mathrm{~nm})$ into the devices and collecting the output with a tapered fiber. The experimental setup was similar to that described in Section 4.1.1. The current was ramped from zero to $250 \mathrm{~mA}$ over several hundred nanoseconds, and the transmitted optical power was acquired using a fast photodetector and an oscilloscope.

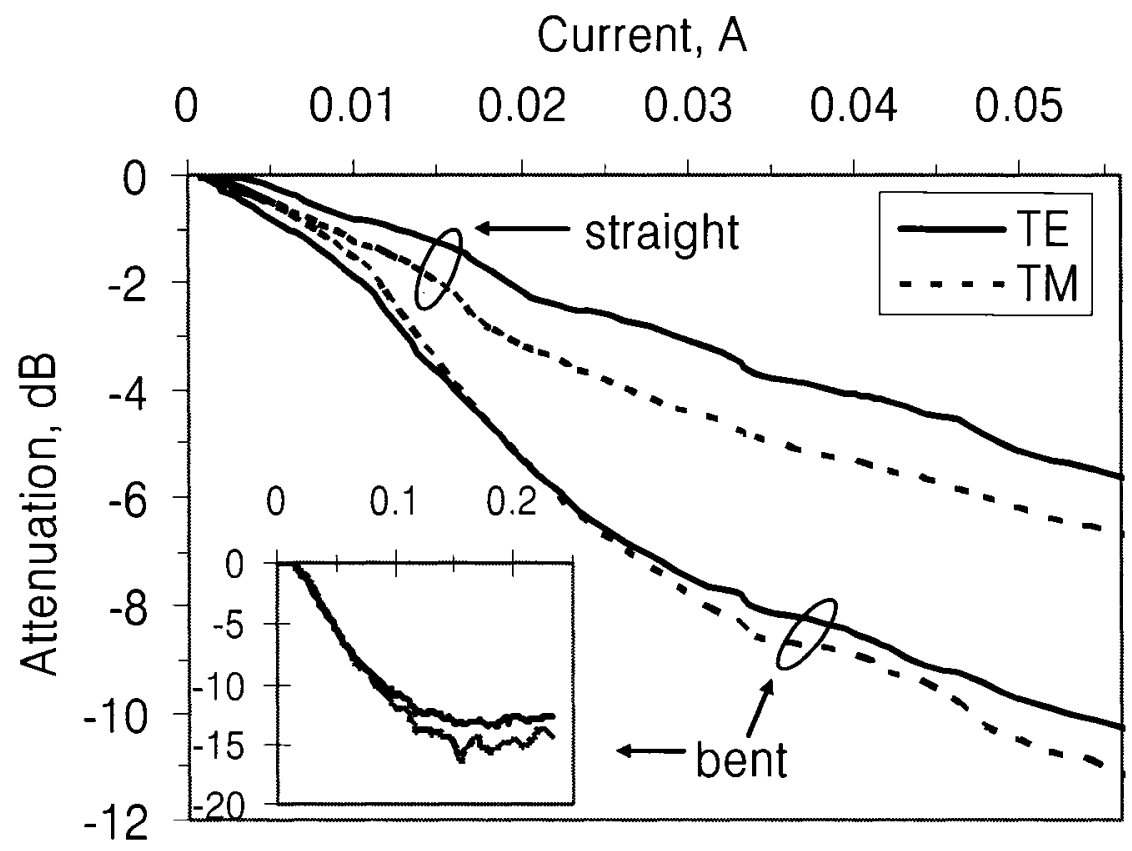

Figure 6-5 Experimental results for straight and bent waveguide attenuators.

The inset shows the bent waveguide data plotted over a larger range.

Experimental measurements of the attenuation versus current for bent and straight waveguide devices are shown in Figure 6-5. As with the BPM simulations, the inclusion of the bend in the device increased the attenuation by about $5 \mathrm{~dB}$ for the same applied current. An index modulation of -0.007 in the InGaAsP core corresponds to an applied current of about 0.055 A [95], so Figure 6-4 and Figure 6-5 can be directly compared. The 2D-BPM model predicts higher attenuation than is achieved experimentally, but both theoretical and experimental results show increased attenuation for the bent attenuator design. At high currents, the maximum measured attenuation is limited to $-15 \mathrm{~dB}$, as seen in the inset of Figure 6-5. This saturation 
effect is due to light that was lost in the active section coupling back into the output waveguide. Further modelling shows that this effect may be reduced, thus enhancing attenuation, by increasing the offset between the input and output waveguide positions. Finally, introduction of a bend in the active attenuator section eliminates most of the measured polarization dependent loss (PDL) of the straight waveguide attenuator.

The BPM results of output power versus index change for $32 \mathrm{~mm}$ radii bend loss attenuators with electrode lengths of $400 \mu \mathrm{m}, 600 \mu \mathrm{m}$, and $800 \mu \mathrm{m}$ are shown in Figure 6-6 (left) and the experimental measurements of the output power versus applied current density for the same devices are shown in Figure 6-6 (right). Both results show that the electrode length may be reduced to about $600 \mu \mathrm{m}$ without much degradation in attenuation.

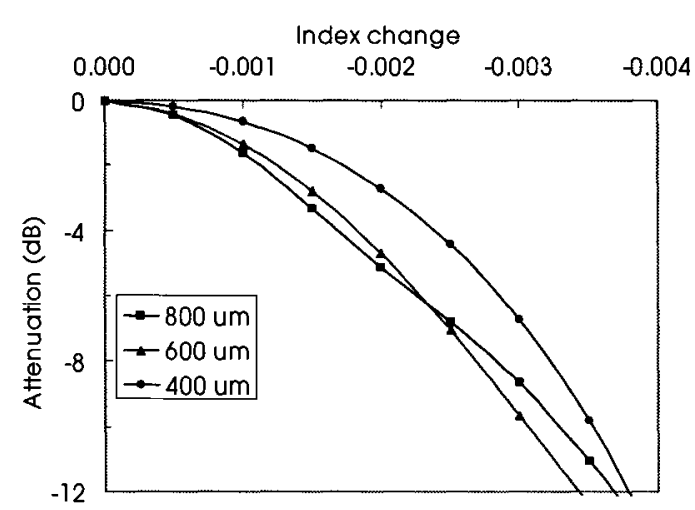

(a)

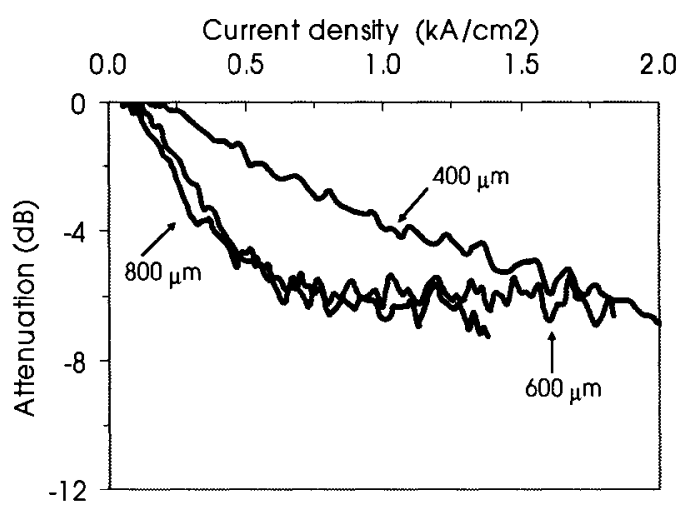

(b)

Figure 6-6 (a) BPM results for bend loss attenuators and (b) experimental results of the same attenuators for $\mathrm{TE}$ polarization.

Additionally, while the experimental data agrees well with theory at low index modulation, the measured attenuation saturates for high index modulation. That is, the BPM effective index model does not reproduce experiments at high index modulation. This may be because the refractive index change was modeled as constant across the width of the waveguide but is in reality a complex and time varying index profile when effects of heating and carrier diffusion are included. 


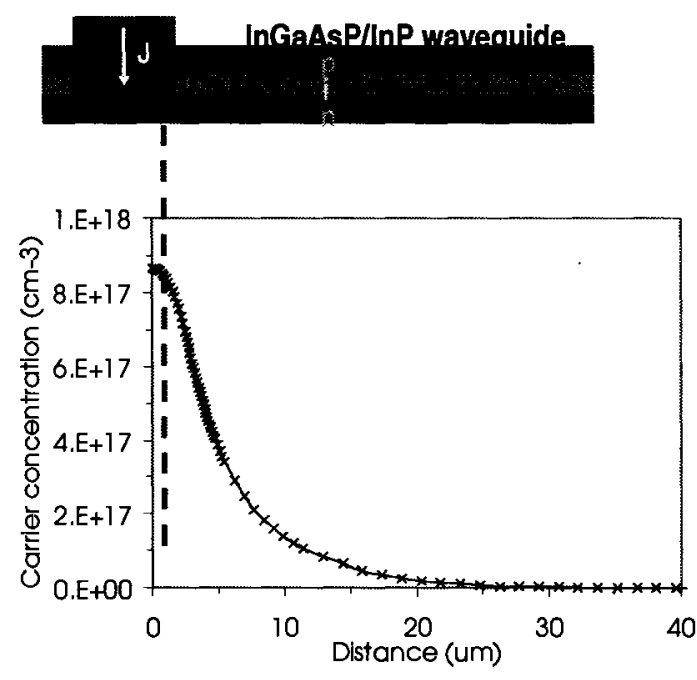

(a)

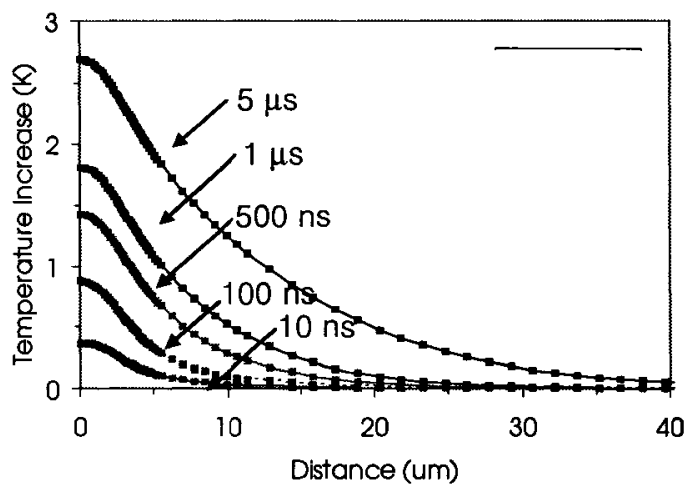

(b)

Figure 6-7 (a) Drift diffusion modeling of carrier concentration across the waveguide, and (b) the corresponding temperature increase due to the thermooptical effect.

Drift diffusion modeling with a $3 \mathrm{~ns}$ carrier time constant and $100 \mathrm{~mA}$ of applied current produces the carrier concentration profile shown in Figure 6-7 (left). Carrier diffusion results in a carrier concentration peak at the center of the waveguide that drops off to either side. Since the carrier induced refractive index change is proportional to carrier concentration $\left(\Delta \mathrm{n}_{\text {elec }} \sim-0.001 \times 10^{17} \times \mathrm{N}\right)$, a more accurate model of index change would follow the profile of carrier concentration. Furthermore, the refractive index change due to the thermo-optical effect is proportional to the change in temperature $\left(\Delta n_{\text {therm }} \sim 0.001 \times \Delta \mathrm{T}\right)$ and so would contribute the time-dependent index change profile shown in Figure 6-7 (b).

Although the 2D effective index models used here are not exact representations of the material structure, they can provide qualitative insight as to how the different contributions to index modification affect device performance. The carrier and thermo-optical effects both modify the effective refractive index $\mathrm{n}_{\text {eff }}$ resulting in the modified index: $\mathrm{n}_{\text {tot }}=\mathrm{n}_{\text {eff }}+\Delta \mathrm{n}_{\text {elec }}+\Delta \mathrm{n}_{\text {therm }}$, however they contribute opposite refractive index changes. Also, while the thermal gradient of 0.5 to 1 Kelvin is set up within several hundred nanoseconds, the carrier concentration is set up much more quickly and so applies at all times plotted in Figure 6-7. The modified index $\mathrm{n}_{\text {tot }}$ due to both effects is shown as a solid line in Figure 6-8 for bias currents of $10 \mathrm{~mA}, 50 \mathrm{~mA}$, and 
$100 \mathrm{~mA}$. The shaded grey area is the unmodified effective index profile, and index modified only by the carrier effect is shown as a dashed line. At low bias, the index profile is similar to the basic model of a constant index change across the width of the waveguide; but at high bias, the index profile in Figure 6-8 is very different from the basic model. In particular, due to carrier diffusion there exist regions of reduced index adjacent to the electrode, which serves to maintain the index step at the edge of the waveguide. Preliminary results with this reduced index region modeled as a $1 \mu \mathrm{m}$ wide constant refractive index depression shows that such an index profile maintains waveguide confinement and so may be the source of the experimentally observed saturation in attenuation. A buried channel waveguide would confine carriers laterally by trapping them in the InGaAsP well. This would significantly decrease carrier diffusion and allow larger attenuation.

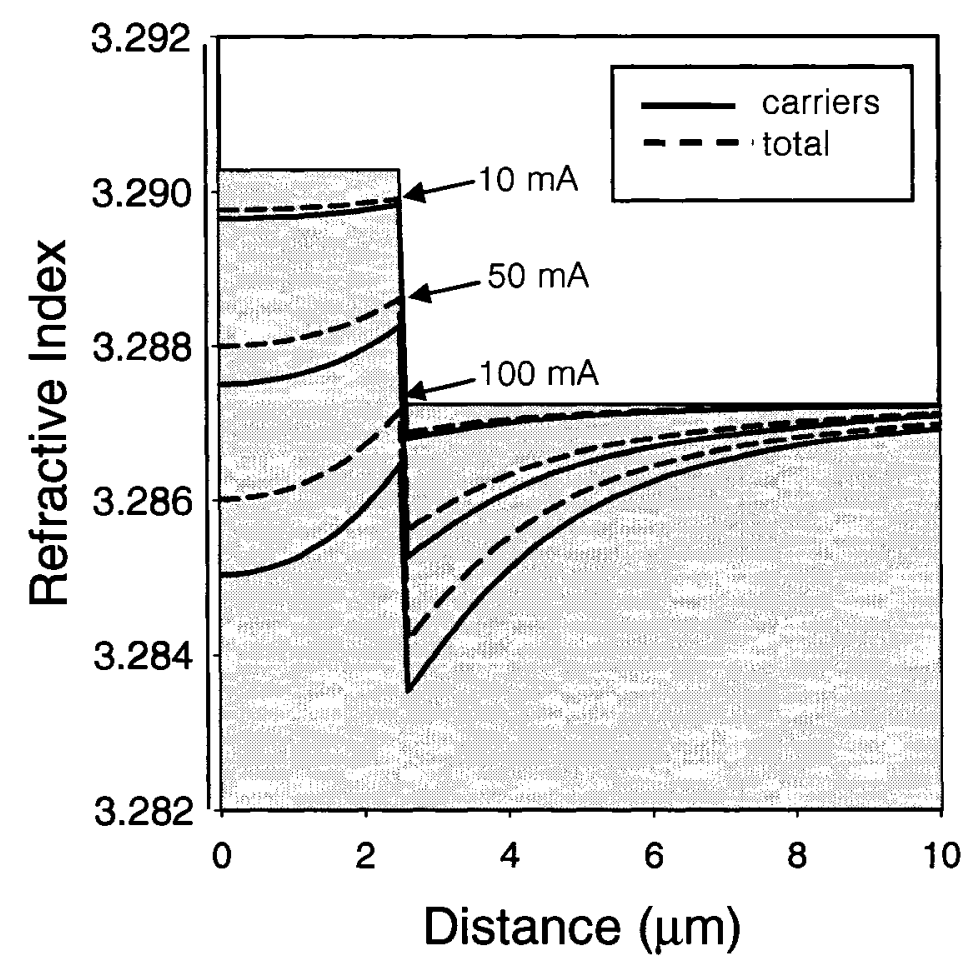

Figure 6-8 Modeling results showing effective refractive index profile due to carrier injection (solid line) and both carrier and thermal (dashed line) effects at applied currents of $10 \mathrm{~mA}, 50 \mathrm{~mA}$, and $100 \mathrm{~mA}$.

In general, theoretical modeling is in agreement with experimental results. For example, we observe that longer electrode lengths require less current density for 
attenuation. The best measured extinction ratio is approximately $15 \mathrm{~dB}$ as expected from modeling. We note however that the current densities required to achieve full modulation are ten times larger than expected from the Mach Zehnder interferometer measurements of carrier induced index change [96]. We suggest that the combined effects of current spreading, carrier diffusion and local heating modify the index profile in the vicinity of the waveguide. As a result, a larger modulation current is required than predicted by the BPM model, which assumes a sharp refractive index step between the ridge waveguide and lateral cladding. Current spreading and carrier diffusion will both broaden the carrier distribution and resulting index profile well beyond the nominal ridge edges. Under forward bias, a localized step like temperature distribution will occur under the waveguide ridge. This local temperature gradient is established within a few hundred nanoseconds [67]. In the case of the bend loss modulator, the resulting positive thermo-optic index change will counteract the carrier induced index suppression, and enhance the waveguide confinement. Thus the thermal gradient may be the cause of the larger current density required for modulation.

The measured bend loss attenuator time response along with the $100 \mathrm{~ns}$ applied pulse is shown in Figure 6-9. The actuation time of the optical pulse is less than $20 \mathrm{~ns}$, disregarding the electrical ringing from the measurement circuit. This is similar to speeds measured from switching devices fabricated on the same wafer, as described previously.

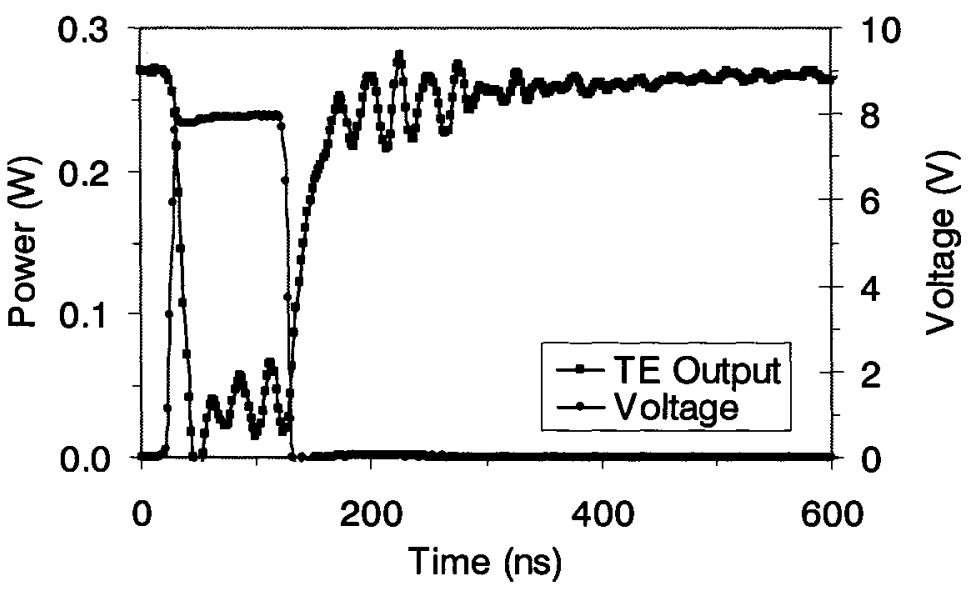

Figure 6-9 Experimental measurements of output power measured from fabricated bend loss attenuator and voltage from pulse generator versus time for an applied pulse length of $100 \mathrm{~ns}$. 


\subsection{Cascaded Switches}

This work was motivated by the demand for an integrable optical switch with an actuation speed on the order of one nanosecond, which would be suitable for use in an optical router operating at the IP or MPLS level. The indium phosphide (InP) material system is ideal for its source and detector functionalities, and carrier injection and electro-optical modulation were identified as actuation mechanisms satisfying nanoseconds speeds.

The $2 \times 2$ switches were designed according to a crosspoint design by Soref et al. [97] and comprised four DOS switches connected as shown in Figure 6-10. They were fabricated on the wafers described in Section 3.3 and tested with the experimental setup described in Section 4.1.1.

With the outer electrodes biased, the light at the two inputs take a path that crosses each other (arrows) to exit at the opposite outputs, i.e. cross configuration (Figure 6-10(a)). With the inner electrodes biased, the light at the two inputs will travel straight across to the output ports, i.e. bar configuration (Figure 6-10(b)).

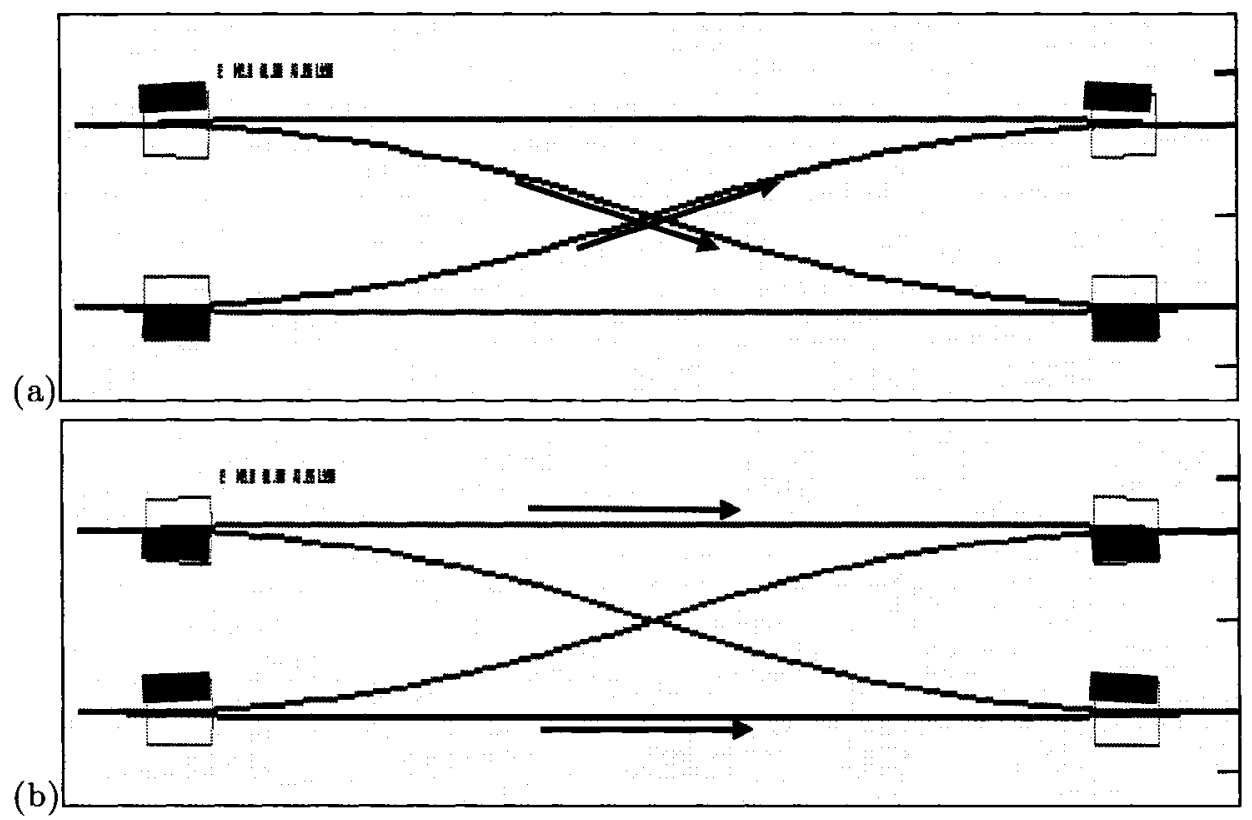

Figure 6-10 Illustration of $2 \times 2$ switch in (a) cross configuration and (b) bar configuration. The solid electrodes indicate (forward) biased electrodes. 
The constituent DOS devices had branching angles of $1.26^{\circ}$ and electrodes lengths of about $340 \mu \mathrm{m}$. The waveguides crossed at an angle of $12.5^{\circ}$ and have a simulated junction loss of $\sim 0.1 \mathrm{~dB}$. The total length of the $2 \times 2$ switch was $6 \mathrm{~mm}$. The ridge waveguides were $5 \mu \mathrm{m}$ wide and the layer structure was identical to the previously described RW-DOS devices. $2 \times 2$ switches were fabricated by both MOCVD and MBE on wafers with core alloys of $\mathrm{Q}=1.2 \mu \mathrm{m}, 1.3 \mu \mathrm{m}$, and $1.4 \mu \mathrm{m}$.

The experimental setup could accommodate only one input fiber and two probes so each optical path was characterized separately. To characterize each optical path, a current pulse was applied to the two appropriate electrodes along an optical path that would configure the $2 \times 2$ switch for cross or bar state. The current pulses for the two electrodes were generated by two pulse generators simultaneously triggered. The output current of each source was adjusted to optimize switching contrast. This required that the bias applied to the output electrode be about half that applied to the input electrode. This may have been due to non-idealities in the fabricated devices.

Figure 6-11 shows the output power for a $2 \times 2$ switch fabricated on an MBE-grown wafer with a $\mathrm{Q}=1.4 \mu \mathrm{m}$ core. The $2 \mathrm{x} 2$ switch was interrogated with TM-polarized light since the transmitted power in TE-polarization was very low. A $200 \mathrm{~ns}$ current pulse of $10 \mathrm{~mA}$ was applied at the input electrode and $5 \mathrm{~mA}$ at the output electrode. All optical path configurations result in switching contrasts of about 7-8 dB. Devices on MOCVD-grown wafers had comparable results. This poor performance in comparison with the single RW-DOS devices is not yet fully understood. Propogation losses are large, as evidenced by the undetectable transmission in TE polarization. Also, as seen in Figure 6-11, the small contrast is largely due to the high crosstalk in the "off" arm, suggesting a significant amount of stray light is coupled back into the output waveguides. This combination of small signal due to large propagation losses and large noise due to coupling of stray light may be the reason for the poor switching contrast observed in the $2 \times 2$ switches.

Cross configuration appears to result in slightly better contrast than bar configuration. The switching times of the trailing edge are about $40 \mathrm{~ns}$, consistent with previous measurements on individual DOS devices, while on the leading edge they are about 15 
ns. This shorter time suggests that the switch is overdriven in forward bias. A negative applied bias may reduce the switching times of the trailing edges.

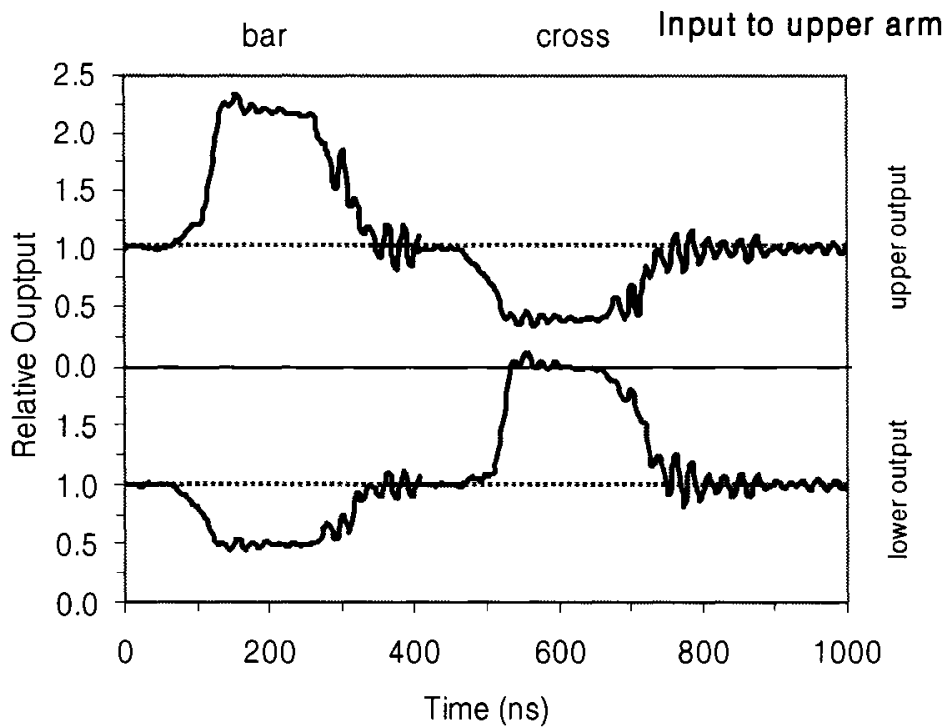

(a)

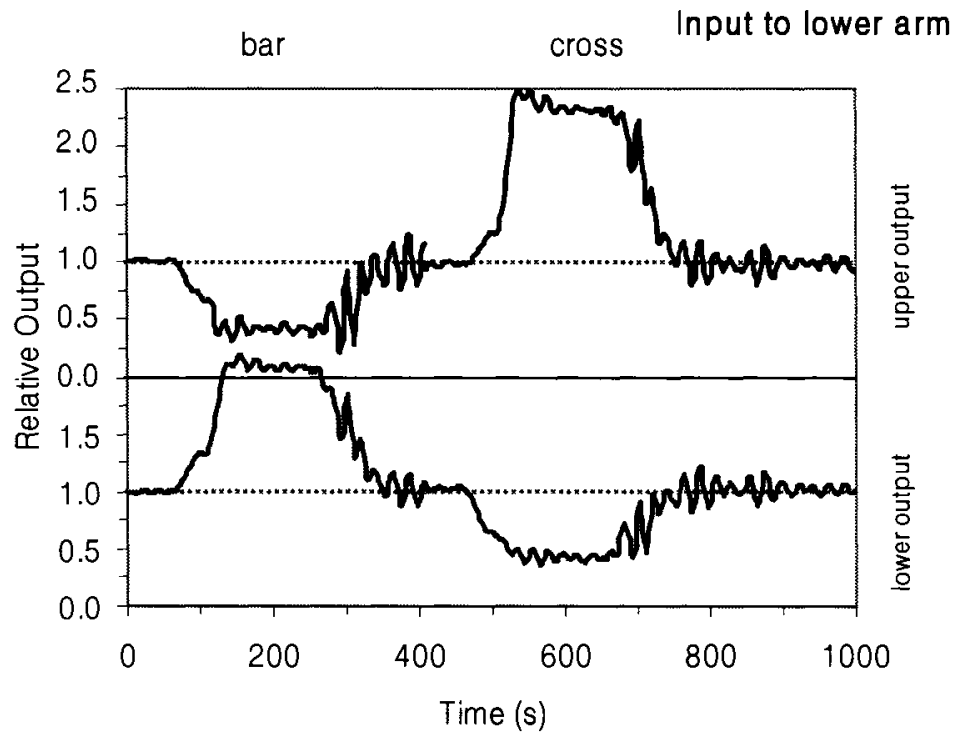

(b)

Figure 6-11 Plots of relative output power from ports of a $2 \times 2$ switch when signal is input to the (a) top arm and (b) bottom arm. Bar and cross configurations are indicated. 


\subsection{Summary}

We have fabricated a bend loss attenuator actuated by carrier injection, with significant improvements in performance over a straight waveguide attenuator. Modulation response times of less than 20 nanoseconds have been observed. Attenuation of $3 \mathrm{~dB}$ is achieved by an actuation current of $\sim 12 \mathrm{~mA}$, which is less than half the current required by a comparable straight waveguide attenuator. The measured polarization dependence of the attenuation is also largely eliminated by the addition of the waveguide bend. We have also integrated four discrete RW-DOS devices into a $2 \times 2$ crosspoint switch. Switching contrast ratios of 7 to $8 \mathrm{~dB}$ have been achieved with actuation currents of $10 \mathrm{~mA}$ and $5 \mathrm{~mA}$ on the input and output electrodes respectively.

Thus we have demonstrated carrier injection actuation in a suite of devices for use as building blocks, as well as initial integration of such devices into a larger system for the development of a burst switching based optical router. 


\section{Chapter 7}

\section{CONCLUSIONS AND FUTURE WORK}

We have presented the first experimental results of carrier-induced switching on a novel Y-splitter digital optical switch design, as well as modeling and measurements of a new bend-loss attenuator. Additionally, we have performed the most detailed study to date of carrier injection in indium phosphide as relates to refractive index modifications in general and the performance of our DOS and attenuator devices in particular. I now summarize the central results of our work, highlighting its significance in the context of the research field, and provide directions for future exploration.

\subsection{Summary and Conclusions}

\subsubsection{Study of Carrier Injection}

The central aim of this work has been the development of fast optical switching and attenuation devices, suitable for integration, based on novel digital optical switch and bend-loss designs, respectively. Actuation by carrier injection was the specific goal, in response to existing requirements for nanosecond actuation times and in view of the 
benefits of carrier injection. It is a timely study given the recent interest in burst and packet switching to emerge from Asia [5].

As such, a detailed study of carrier injection, including experimental comparison with theory and an investigation of material parameters, is vital to the proper design of carrier injection actuated devices. While there do exist several reports on carrierinjection dynamics in relation to laser performance, there are significantly fewer studies with respect to switching devices. This dearth of experimental data has instigated recent publications in this area by research groups such as ours investigating carrier-injection switching $[74,75]$. These studies demonstrate the importance of understanding carrier-injection dynamics to the development of optical switching devices.

The study of carrier injection we presented in Chapter 4 is the most comprehensive one published up to now. A comparison of different models of carrier injection induced index modification with the available experimental data, including our own, has provided support for the model of bandgap shrinkage according to Botteldooren and Baets [60]. Our experimental results agree with previously published values and theoretical modeling and as such present a good summary of the material property. We conclude from our study that the smaller bandgap material $(\mathrm{Q}=1.4 \mu \mathrm{m})$ may be used to reduce actuation power without significant detriment to device loss, and indeed may benefit switching contrast as well. Moreover, the study of carrier recombination provides insight into the tradeoffs between various aspects of switching performance, including not only switching speed and switching power, but also switching contrast, through the effects of carrier lifetime and diffusion on the refractive index profile. The demonstration of increasing switching speed through overdriving, however, provides a means of decoupling this parameter.

\subsubsection{Device Results}

I have presented results in Chapters 5 and 6 on optimization of the DOS device design, fabrication, and operation. The DOS devices are novel in their optical design, and this thesis has produced working devices. Improvements have been made to the layer structure, device design, and actuation mechanism to reduce switching power and 
actuation speed. The device is at the forefront of integrated optical switching research in terms of speed, size, and power requirements. Furthermore, we have successfully demonstrated the integration of four discrete switches into a $2 \times 2$ structure.

We have demonstrated switching contrast ratios of better than $25 \mathrm{~dB}$ in both polarizations. Driving power has been reduced by one order of magnitude over the course of this work, to less than $20 \mathrm{~mW}$ magnitude through optimization of wafer and device design. Further reduction in switching current, at the expense of switching speed, is possible through use of material with lower defect density. This tradeoff between current and speed is partially averted by overdriving the current pulses to enhance switching speed. In this way we have demonstrated switching times of less than $7 \mathrm{~ns}$, limited by our experimental setup.

The comparison of methods of electrical isolation has not previously been addressed and provides electrical as well as optical modeling and experimental results that may be applied to other switch designs. In particular, electrical results show that the etching of a trench is equally effective in electrical isolation and simpler than ion implantation in terms of fabrication. However, our switch design, being particularly sensitive to the shape of the index modulation region and therefore amount of carrier diffusion, precluded the use of the straight trench because of the decreased optical performance. Still, for switch designs that are not as sensitive to carrier diffusion, our results suggest that an etched trench is a viable option for electrical isolation.

Finally, a novel bend loss modulator design has been modeled, designed, and tested. This simple bent waveguide design may be implemented in existing connection waveguides and reduces power requirements compared to a straight waveguide design, both desirable characteristics for these ubiquitous devices. 


\subsection{Original Contributions}

The major original contributions of the current study consist of:

- a thorough study of the carrier injection mechanism in the InGaAsP/InP material system, that allows for the optimization of switch design, including:

- BPM, Menu, and Matlab simulation and design of Mach-Zehnder and Fabry-Perot interferometer test structures,

- layout of mask,

- participated in process development with Dr. Barrios, who fabricated the structures,

- testing and characterization of wafer material and devices,

- data analysis,

- Matlab simulation and modeling, building on the work of S. Abdalla, with the help of Dr. Janz and Dr. McKinnon, of carrier induced refractive index modifications and carrier lifetimes, and

- the experimental demonstration of optimized DOS and VOA devices using carrier injection and electro-optical switching mechanisms, including:

- BPM and Matlab simulation and design of wafer layer structures as well as RW-DOS devices (based on a design by Dr. Dêlage and Dr. He), bend loss modulators, and $2 \times 2$ switches,

- layout of mask,

- participated in process development with Dr. Barrios, who fabricated the structures, 
- testing and characterization of devices,

- data analysis.

A number of publications in refereed journals and conference proceedings resulted from this study:

S. Ng, S. Janz, W.R. McKinnon, P. Barrios, A. Delâge and B. A. Syrett, "Performance optimization of a reconfigurable waveguide digital optical switch on InGaAsP/InP: Design, Material, and Carrier Dynamics", submitted to IEEE Journal of Quantum Electronics..

S. Ng, S. Janz, W.R. McKinnon, P. Barrios, A. Delâge and B. A. Syrett, "Performance optimization of a reconfigurable waveguide digital optical switch on InGaAsP/InP", LEOS 2006, October/November 2006, WBB3.

S. Ng, S. Abdalla, B.A. Syrett, P. Barrios, W. R. McKinnon, A. Delâge, I. Golub, S. Janz, and J. Lapointe, "Electrical Isolation of Electrodes with Submicron Separation in a Digital Optical Switch", Journal of Vacuum Science 6 Technology A Vacuum, Surfaces, and Films, May/June 2006, 24(3), pp. 807-11.

S. Ng, S. Abdalla, P. Barrios, A. Delâge, S. Janz, W.R. McKinnon, B. A. Syrett, "Bend loss attenuator by carrier injection in InGaAsP/InP", Electronics Letters, November 2005, 41(24), pp. 1348-50.

S. Ng, S. Abdalla, B. A. Syrett, P. Barrios, A. Delâge, S. Janz, W.R. McKinnon, "Fast Bend Loss Attenuator Using Carrier Injection", OSA Integrated Photonics Research and Applications, April 2005, IWA5.

P. Barrios, S. Janz, A. Delâge, I. Golub, W. R. McKinnon, P. Poole, S. Abdalla, D. Celo, S. Ng, T.J. Smy, B. A. Syrett, "Digital optical switches with reconfigurable output waveguide branches in InP", Integrated Optical Devices, Nanostructures, and Displays, Oct 2004, Proc. SPIE 5622, pp. 751-4. 
S. Ng, S. Abdalla, P. Barrios, A. Delâge, S. Janz, W.R. McKinnon, P. Poole, B. A. Syrett, "Low current optical switching by carrier injection induced reconfigurable waveguiding", Photonic Applications in Telecommunications, Sensors, Software, and Lasers, Sept 2004, Proc. SPIE 5579, pp. 644-651.

S. Ng, S. Abdalla, B. A. Syrett, P. Barrios, A. Delâge, I. Golub, J.-J. He, S. Janz, W.R. McKinnon, P. Poole, "Improved digital optical switching using carrier injection induced reconfigurable waveguides", OSA Integrated Photonics Research Technical Digest, June 2004.

S. Abdalla, S. Ng, P. Barrios, D. Celo, S. El Mougy, A. Delâge, I. Golub, J.-J. He, S. Janz, W. R. McKinnon, P. Poole, S. Raymond, T.J. Smy, B. A. Syrett, "Optical switching in InGaAsP waveguides using localized index gradients", Journal of Vacuum Science \& Technology A Vacuum, Surfaces, and Films, May 2004, 22(3), pp. 796-9.

S. Abdalla, S. Ng, P. Barrios, D. Celo, A. Delâge, S. El Mougy, I. Golub, J.-J. He, S. Janz, W. R. McKinnon, P. Poole, S. Raymond, T.J. Smy, B. A. Syrett, "Carrier injection-based digital optical switch with reconfigurable output waveguide arms", IEEE Photonics Technology Letters, April 2004, 16(4), pp. 1038-40.

S. Janz, P. Barrios, A. Delâge, I. Golub, J.-J. He, W. R. McKinnon, P. Poole, S. Abdalla, D. Celo, S. El Mougy, S. Ng, B. A. Syrett, "Digital optical switch based on fast thermal gradient switching in InGaAsP waveguides", Integrated Photonics Research Trends in Optics and Photonics Series, Vol.91 2003, pp. 92-4.

\subsection{Directions of Future Research}

Research on the DOS design can be extended in several directions. One such direction is to focus on directing performance and packaging of the current design towards commercial applications. In terms of novel research, the development of the higher speed electro-optical quantum well designs requires significant redesign and experimen- 
tal validation; as would exploration of the thermo-optical gradient effect on silicon-oninsulator (SOI), leading to its application to switching.

\subsubsection{Improvements to InGaAsP/InP RW-DOS Devices}

Several improvements beg immediate attention for development of the existing switch device towards commercial applications. One is implementation of the device using buried waveguides, which may eliminate the birefringence that results from the asymmetry of the ridge waveguide design. Also, characterization of loss and contrast is currently hampered by issues of repeatability in fiber coupling and variation in operating conditions such as temperature, humidity, and vibration. Connectorization and proper packaging of the devices would improve performance, permit environmental testing, and allow more accurate characterization.

For the current DOS design with a bulk InGaAsP core, more detailed 3D BPM simulations would confirm many of the results that have been insinuated by the simple $2 \mathrm{D}$ effective index modeling thus far. First, a 3D BPM simulation could confirm the role of the difference in vertical confinement to the variation in switch performance between the three alloys. Second, including the full refractive index profile as determined from drift-diffusion models of carrier concentration distributions, could confirm the role of diffusion in degrading switching and attenuation performance.

For the DOS design with quantum well cores, experiments should be repeated with wafers whose band gaps are closer to specifications, to allow operation at $1.55 \mu \mathrm{m}$ in line with standard telecommunications wavelengths. More importantly, quantum well structures that allow the QCSE to induce negative changes in refractive index [98] should be explored to optimize DOS performance.

For both bulk and QW devices, the electrical contact pads should be redesigned for high speed operation. For example, high speed probes generally have at least one adjacent ground point in addition to the signal point to reduce signal distortions. Thus to use such probes, the devices must be redesigned with ground pads placed adjacent to each active pad. Additionally, the size and shape of the contact pads should be redesigned to reduce capacitance. 
Finally, a buried channel waveguide structure could be explored to reduce the polarization dependence that results from the ridge waveguide structure. The lateral energy step due to the heterojunction would also help to reduce lateral current spreading, thereby making the index profile steeper and potentially enhancing switching contrast. Such a buried structure would require regrowth or ion implantation to form buried guiding and current blocking structures as illustrated in Figure 7-1.

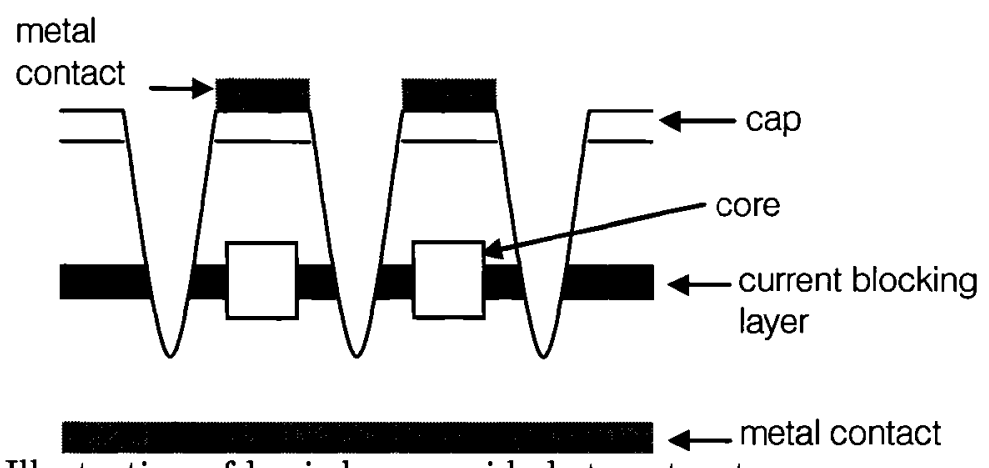

Figure 7-1 Illustration of buried waveguide-heterostructure.

\subsubsection{Optical Switches in SOI}

The thermal gradient switching observed in the original set of fabricated DOS can be applied to devices fabricated on silicon-on-insulator (SOI). SOI is a desirable material platform because it is a high index contrast material. This allows light to be confined to narrow waveguides and through tight bending radii, resulting in smaller device footprint. In addition, SOI is used for digital integrated circuits and so wafers are readily available and compatible with CMOS technology.

Switch devices exploiting the thermal gradient effect have been modeled with 2D BPM software. A mask has been prepared that includes a variety of test structures to characterize and optimize device designs for the thermal gradient effect. These structures include, for example, MZIs with one or two heaters that are laterally offset by varying distances from one or both interferometer arms, respectively. Such a structure will allow the lateral profile of the thermal gradient to be characterized so that the optimum heater position may be determined. I have also included DOS, MZ, DC and MMI switch designs. Other test structures designed by K. P. Yap include 
waveguide mode converters, Van de Pauw's structures to measure sheet resistance of potential heater and electrode metals, photoresist erosion monitoring structures, and trenches for etching of optical facets.

Thus characterization of devices on the current mask will allow a mapping of parameters such as the thermal gradient profile, from which optimized designs of switch and attenuator structures may be extracted. 


\section{Appendix}

\section{Calculation of Crystal Orientation}

InGaAsP is a cubic $\overline{4} 3 \mathrm{~m}$ crystal with $\mathrm{n}_{1}=\mathrm{n}_{2}=\mathrm{n}_{3}=\mathrm{n}$ and the following Pockel's coefficients:

$$
r_{i j k}=r_{I k}=\left[\begin{array}{ccc}
0 & 0 & 0 \\
0 & 0 & 0 \\
0 & 0 & 0 \\
r_{41} & 0 & 0 \\
0 & r_{41} & 0 \\
0 & 0 & r_{41}
\end{array}\right] \text { where } I=[i, j]=\left[\begin{array}{ccc}
1 & 6 & 5 \\
6 & 2 & 4 \\
5 & 4 & 3
\end{array}\right]
$$

The modified index ellipsoid describes refractive indices with an electric field:

$$
\sum_{i j} n_{i j}(\vec{E})=n_{i j}+\sum_{k} r_{i j k} E_{k}
$$

Wafers are grown with (100) surface normal. Waveguides are often oriented along the primary axis (011) to take advantage of the resultant "V" etch for grooves. Whereas structures positioned along the secondary axis (011) will be subjected to a "dovetail" etch.

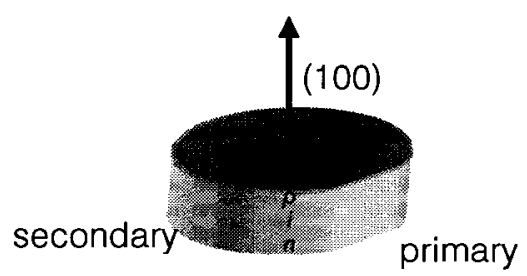

An electric field applied in the direction of the surface normal $\vec{E}=(E, 0,0)$ reverse biases the pin diode. For such a field, the only applicable non-zero terms are $\mathbf{r}_{321}$ and $\mathrm{r}_{231}=\mathrm{r}_{41}$. A rotation of the coordinate system to the primary $u_{1}$ and secondary $u_{2}$ axes results in the following index ellipsoid: 


$$
\frac{x_{1}^{2}}{n^{2}}+u_{1}^{2}\left(\frac{1}{n^{2}}+r_{41} E\right)+u_{2}^{2}\left(\frac{1}{n^{2}}-r_{41} E\right)=1
$$

Assuming a small electro-optically induced index change, the modified refractive indices along the primary and secondary axes are approximately $n^{2}\left(1-1 / 2 r_{41} E n^{2}\right)$ and $n^{2}\left(1+1 / 2 r_{41} E n^{2}\right)$ respectively. Thus for the electric field to increase refractive index, the waveguides must be oriented along the secondary axis. 


\section{References}

[1] G. I. Papadimitriou, C. Papazoglou, and A. S. Pomportsis, "Optical switching: switch fabrics, techniques, and architectures," Journal of Lightwave Technology, vol. 21, pp. 384-405, 2003.

[2] "Dell'Oro Reports on Optical," vol. 2006: Lightreading, 2005, pp. http://www.lightreading.com/document.asp?doc_id $=84551$.

[3] J. J. He and A. Delâge, "Digital Optical Switch," U. Patent, Ed., 2004.

[4] "The Future of Optical Switching: a Market Forecast, 2002-2006," vol. 2005, 2002, pp. http://www.cir-inc.com/resources/mag_detail.cfm?MID=53.

[5] S. Hardy, "Companies place their faith in optical packet switching," vol. 2006, 2005, pp.

http://lw.pennnet.com/articles/article_display.cfm?article_id=242492.

[6] R. Luijten, W. E. Denzel, R. R. Grzybowski, and R. Hemenway, "Optical interconnection networks: The OSMOSIS project," presented at Lasers and Electro-Optics Society, 2004. The 17th Annual Meeting of the IEEE, 2004.

[7] B. E. A. Saleh and M. C. Teich, Fundamentals of Photonics. New York: Wiley \& Sons, Inc., 1991.

[8] W. Yuan, S. Kim, H. R. Fetterman, W. H. Steier, R. Dinu, and J. Danliang, "Hybrid Integrated Electrooptic Polymeric Digital Optical Switches (DOS's) with Lower Loss," presented at Optical Fiber Communication Conference, 2006 and the 2006 National Fiber Optic Engineers Conference, Anaheim, California, 2006.

[9] Y. Tsay, B. Bendow, and S. S. Mitra, "Theory of the temperature derivative of the refractive index in transparent crystals," Physical Review B Solid State, vol. 8, pp. 2688-96, 1973.

[10] T. Nakagami and K. Nakatsuhara, "Optical routing switches incorporating ferroelectric liquid crystals for use in photonic networks (invited paper)," Proceedings of the SPIE The International Society for Optical Engineering, vol. 5741 , pp. 98-106, 2005.

[11] J. Xiao Qing, Y. Jian Yi, Z. Heng Zheng, C. Ke Jian, T. Yi, L. Xi Hua, and W. Ming Hua, "Photon-induced total-internal-reflection all-optical switches," IEEE Photonics Technology Letters, vol. 16, pp. 443-5, 2004. 
[12] "All-Optical Switching in Silicon by Carrier Injection," in 2003 Material Research at MIT, vol. 2005: MIT, 2003, pp. http://mpcweb.mit.edu/about_mpc/ar2003/kaertner.pdf.

[13] K. Campbell, A. Groisman, U. Levy, P. Lin, S. Mookherjea, D. Psaltis, and Y. Fainman, "A microfluidic 2x2 optical switch," Applied Physics Letters, vol. 85, pp. 6119-21, 2004.

[14] H. E. Horng, C. S. Chen, K. L. Fang, S. Y. Yang, J. J. Chieh, H. Chin Yih, and H. C. Yang, "Tunable optical switch using magnetic fluids," Applied Physics Letters, vol. 85, pp. 5592-4, 2004.

[15] Q. Li, P. W. E. Smith, B. J. Robinson, and D. A. Thompson, "An ultrafast alloptical asymmetric Fabry-Perot switch based on bulk Be-doped InGaAsP grown by He-plasma-assisted epitaxy," Optical and Quantum Electronics, vol. 33, pp. 1055-62, 2001.

[16] T. Yasuda, Y. Tsuji, and M. Koshiba, "Tunable light propagation in photonic Crystal coupler filled with liquid Crystal," IEEE Photonics Technology Letters, vol. 17, pp. 55-7, 2005.

[17] J. Chang Hyeon, Y. Youngjoo, C. Junghoon, K. Seong Hyok, and B. Jong Uk, "Electromagnetic 2x2 MEMS optical switch," IEEE Journal of Selected Topics in Quantum Electronics, vol. 10, pp. 545-50, 2004.

[18] M. Simard, Z. Khalid, and A. G. Kirk, "Digital optical space switch based on micromotor grating scanners," IEEE Photonics Technology Letters, vol. 18, pp. $313-15,2006$.

[19] R. Helkey, S. Adams, J. Bowers, T. Davis, O. Jerphagnon, V. Kaman, A. Keating, L. Bin, C. Pusarla, D. Xu, Y. Shifu, and Z. Xuezhe, "Design of large, MEMS-based photonic switches," Optics and Photonics News, vol. 13, pp. 40-3, 2002.

[20] "Optical Switches: Making Optical Networks a Brilliant Reality," vol. 2005, pp. http://www.iec.org/online/tutorials/opt_switch/topic04.html.

[21] D. T. Fuchs, C. R. Doerr, V. A. Aksyuk, M. E. Simon, L. W. Stulz, S. Chandrasekhar, L. L. Buhl, M. Cappuzzo, L. Gomez, A. Wong Foy, E. Laskowski, E. Chen, and R. Pafchek, "A hybrid MEMS-waveguide wavelength selective cross connect," IEEE Photonics Technology Letters, vol. 16, pp. 99-101, 2004.

[22] M. W. Pruessner, K. Amarnath, M. Datta, D. P. Kelly, S. Kanakaraju, H. Ping Tong, and R. Ghodssi, "InP-based optical waveguide MEMS switches with eva- 
nescent coupling mechanism," Journal of Microelectromechanical Systems, vol. 14, pp. 1070-81, 2005.

[23] R. Guerre, F. Fahrni, and P. Renaud, "Fast 10- mu s microelectromechanical optical switch inside a planar hollow waveguide (PHW)," Journal of Lightwave Technology, vol. 24, pp. 1486-98, 2006.

[24] T. Kanie, M. Katayama, T. Sano, H. Kohda, T. Sunaga, M. Shiozaki, M. Furukawa, H. Suganuma, K. Saitoh, and M. Nishimura, "Ultra-compact multichannel optical components based on PLC technologies," presented at OFC 2003 Optical Fiber Communication Conference and Exhibition. Atlanta, GA, USA. IEEE/Communications Soc. IEEE/Lasers \& Electro Opt. Soc. Opt. Soc. America. 2328 March 2003., 2003.

[25] J. G. Fenn, M. Mazilu, A. G. Iglesias, and A. Miller, "Ultrafast dynamics in semiconductor optical amplifiers with implications for interferometric switching," presented at Lasers and Electro-Optics Society, 2002. The 15th Annual Meeting of the IEEE, 2002.

[26] S. S. Agashe, K. T. Shiu, and S. R. Forrest, "Compact polarization-insensitive InGaAsP-InP 2 × 2 optical switch," IEEE Photonics Technology Letters, vol. 17, pp. 52-4, 2005.

[27] W. Fan, Y. Jianyi, C. Limei, J. Xiaoqing, and W. Minghua, "Optical switch based on multimode interference coupler," IEEE Photonics Technology Letters, vol. 18, pp. 421-3, 2006.

[28] T. Ogawa, N. Yamamoto, Y. Watanabe, K. Komori, M. Itoh, and T. Yatagai, "The design method of a photonic crystal directional coupler switch with short switching length and wide bandwidth," Proceedings of the SPIE The International Society for Optical Engineering, vol. 5733, pp. 377-85, 2005.

[29] R. Prasanth, J. E. M. Haverkort, and J. H. Wolter, "Compact polarizationindependent Mach-Zehnder space switch combining carrier depletion and the quantum confined Stark effect," IEEE Journal of Quantum Electronics, vol. 39, pp. 379-83, 2003.

[30] U. Fischer, B. Schuppert, and K. Petermann, "Optical waveguide switches in silicon based on Ge-indiffused waveguides," IEEE Photonics Technology Letters, vol. 6, pp. 978-80, 1994.

[31] K. Chong Siew and S. Shaari, "Polymer thermooptic switch for C-band based on multimode interference Mach-Zehnder interferometer," presented at 2004 IEEE International Conference on Semiconductor Electronics. Kuala Lumpur, Malaysia. 79 Dec. 2004., 2004. 
[32] X. Jinsong, Y. Jinzhong, W. Zhangtao, F. Zhongchao, and C. Shaowu, "Low power $2 \times 2$ thermo-optic SOI waveguide switch fabricated by anisotropy chemical etching," Optics Communications, vol. 232, pp. 223-8, 2004.

[33] H. Nakamura, Y. Sugimoto, K. Kanamoto, N. Ikeda, Y. Tanaka, Y. Nakamura, S. Ohkouchi, Y. Watanabe, K. Inoue, H. Ishikawa, and K. Asakawa, "Ultra-fast photonic crystal/quantum dot all-optical switch for future photonic networks," Optics Express. 27 Dec. 2004; 12(26), 2004.

[34] S. Boscolo and K. J. Blow, "Interferometric optical switch enhanced by a multiresonance ring resonator structure," Journal of Modern Optics, vol. 52, pp. 845-55, 2005.

[35] W. M. J. Green, R. K. Lee, G. A. DeRose, A. Scherer, and A. Yariv, "Hybrid InGaAsP-InP Mach-Zehnder racetrack resonator for thermooptic switching and coupling control," Optics Express. 7 March 2005; 13(5), 2005.

[36] V. R. Almeida, C. A. Barrios, R. R. Panepucci, M. Lipson, M. A. Foster, D. G. Ouzounov, and A. L. Gaeta, "All-optical switch on a silicon chip," presented at Conference on Lasers and Electro Optics (CLEO). San Francisco, CA, USA. APS. IEEE. Opt. Soc. of America. 1621 May 2004., 2004.

[37] C. A. Barrios, "High-performance all-optical silicon microswitch," Electronics Letters, vol. 40, pp. 862-3, 2004.

[38] V. Van, T. A. Ibrahim, K. Ritter, P. P. Absil, F. G. Johnson, R. Grover, J. Goldhar, and P. T. Ho, "All-optical nonlinear switching in GaAs-AlGaAs microring resonators," Photonics Technology Letters, IEEE, vol. 14, pp. 74, 2002.

[39] I. Kiyat, A. Aydinli, and N. Dagli, "Low-power thermooptical tuning of SOI resonator switch," IEEE Photonics Technology Letters, vol. 18, pp. 364-6, 2006.

[40] Y. Silberberg, P. Perlmutter, and J. E. Baran, "Digital optical switch," Applied Physics Letters, vol. 51, pp. 1230-1232, 1987.

[41] W. K. Burns, "Shaping the digital switch," IEEE Photonics Technology Letters, vol. 4 , pp. $861-3,1992$.

[42] H. Okayama and M. Kawahara, "Reduction of voltage-length product for Ybranch digital optical switch," Journal of Lightwave Technology, vol. 11, pp. $379-87,1993$. 
[43] N. Keil, H. H. Yao, and C. Zawadzki, "(2x2) digital optical switch realised by low cost polymer waveguide technology," Electronics Letters, vol. 32, pp. 1470-1, 1996.

[44] M. N. Khan, B. I. Miller, E. C. Burrows, and C. A. Burrus, "Crosstalk-, loss-, and length-reduced digital optical Y-branch switches using a double-etch waveguide structure," IEEE Photonics Technology Letters, vol. 11, pp. 1250-2, 1999.

[45] L. Baojun and C. Soo Jin, "2x2 optical waveguide switch with bow-tie electrode based on carrier-injection total internal reflection in SiGe alloy," IEEE Photonics Technology Letters, vol. 13, pp. 206-8, 2001.

[46] C. Sato, S. Iio, M. Suehiro, S. Nakajima, Y. Asano, S. Oka, T. Fujita, H. Kodaka, S. Kobayashi, K. Ikezawa, M. Wada, T. Yakihara, H. Matsuura, and A. Miura, "High-speed waveguide switches for optical routers and networks," presented at Optical Fiber Communication Conference, 2004., Anaheim, CA, 2004.

[47] A. Miura, H. Matsuura, M. Wada, T. Yakihara, H. Kodaka, and K. Ikezawa, "Photonic measurement and control technologies," in Yokogawa Technical Report English Edition, No. 41, vol. 2006, 2006, pp.

http://www.yokogawa.com/rd/pdf/TR/rd-tr-r00041-005.pdf.

[48] T. C. Kowalczyk, I. Finkelshtein, M. Kouchnir, Y. C. Lee, A. D. Nguyen, D. Vroom, and W. K. Bischel, "Polymeric variable optical attenuator array," presented at CLEO 2001. Technical Digest. Summaries of papers presented at the Conference on Lasers and Electro Optics. Postconference Technical Digest. Baltimore, MD, USA. IEEE/Lasers \& Electro Opt. Soc. OSA Opt. Soc. America. Quantum Electron. Division of the Eur. Phys. Soc. Opt. Soc. Japanese Quantum Electron. Joint Group. 611 May 2001., 2001.

[49] N. Young Ouk, L. Chul Hee, K. Jong Min, H. Wol Yon, W. Yong Hyub, L. Hyung Jong, H. Seon Gyu, and O. Min Cheol, "Polymer waveguide variable optical attenuator and its reliability," Optics Communications, vol. 242, pp. 533$40,2004$.

[50] G. Z. Xiao, Z. Zhiyi, and C. P. Grover, "A variable optical attenuator based on a straight polymer-silica hybrid channel waveguide," IEEE Photonics Technology Letters, vol. 16, pp. 2511-13, 2004.

[51] Y. Enami, M. Kawazu, A. K. Y. Jen, G. Meredith, and N. Peyghambarian, "Polarization-insensitive transition between sol-gel waveguide and electrooptic polymer and intensity modulation for all-optical networks," Journal of Lightwave Technology, vol. 21, pp. 2053-60, 2003. 
[52] G. J. Veldhuis, F. N. Krommendijk, and P. V. Lambeck, "Integrated optic intensity modulator based on a bent channel waveguide," Optics Communications, vol. 168, pp. 481-91, 1999.

[53] S. M. Garner and S. Caracci, "Variable optical attenuator for large-scale integration," IEEE Photonics Technology Letters, vol. 14, pp. 1560-2, 2002.

[54] S. Tomljenovic Hanic and W. Krolikowski, "New design for a variable optical attenuator based on a bent channel waveguide," Applied Physics B Lasers and Optics, vol. B77, pp. 19-23, 2003.

[55] R. Krahenbuhl, M. M. Howerton, J. Dubinger, and A. S. Greenblatt, "Performance and modeling of advanced Ti:LiNbO/sub 3/ digital optical switches," Journal of Lightwave Technology, vol. 20, pp. 92-9, 2002.

[56] A. Sneh, J. E. Zucker, B. I. Miller, and L. W. Stulz, "Polarization-insensitive InP-based MQW digital optical switch," IEEE Photonics Technology Letters, vol. 9, pp. 1589-91, 1997.

[57] Z. Ce Zhou, C. Ai Hua, E. K. Liu, and G. Z. Li, "Silicon-on-insulator asymmetric optical switch based on total internal reflection," IEEE Photonics Technology Letters, vol. 9, pp. 1113-15, 1997.

[58] J. P. Weber, "Optimization of the carrier-induced effective index change in InGaAsP waveguides-application to tunable Bragg filters," IEEE Journal of Quantum Electronics, vol. 30, pp. 1801-16, 1994.

[59] B. R. Bennett, R. A. Soref, and J. A. Del Alamo, "Carrier-induced change in refractive index of InP, GaAs and InGaAsP," IEEE Journal of Quantum Electronics, vol. 26, pp. 113-22, 1990.

[60] D. Botteldooren and R. Baets, "Influence of band-gap shrinkage on the carrierinduced refractive index change in InGaAsP," Applied Physics Letters, vol. 54, pp. 1989-91, 1989.

[61] C. H. Henry, R. A. Logan, and K. A. Bertness, "Spectral dependence of the change in refractive index due to carrier injection in GaAs lasers," Journal of Applied Physics, vol. 52, pp. 4457-4461, 1981.

[62] A. Lim and R. Gupta, "Blue-Shifting Optical Modulators," vol. 2005, pp. http://www.eng.hull.ac.uk/research/opto/research.html. 
[63] "Nanostructure Optoelectronics," vol. 2005, pp.

http://www.ece.gatech.edu/research/nanostructure_optoelectronics/overview.h tml.

[64] N. Yoshimoto, Y. Shibata, S. Oku, S. Kondo, and Y. Noguchi, "Design and demonstration of polarization-insensitive Mach-Zehnder switch using a latticematched InGaAlAs/InAlAs MQW and deep-etched high-mesa waveguide structure," Journal of Lightwave Technology, vol. 17, pp. 1662-8, 1999.

[65] H. Mohseni, H. An, Z. A. Shellenbarger, M. H. Kwakernaak, and J. H. Abeles, "Enhanced electro-optic effect in GaInAsP-InP three-step quantum wells," $A p$ plied Physics Letters, vol. 84, pp. 1823-5, 2004.

[66] J. E. Zucker, T. Y. Chang, M. Wegener, N. J. Sauer, K. L. Jones, and D. S. Chemla, "Large refractive index changes in tunable-electron-density InGaAs/InAlAs quantum wells," IEEE Photonics Technology Letters, vol. 2, pp. 29-31, 1990.

[67] S. Abdalla, S. Ng, D. Celo, S. El Mougy, T. Smy, B. Syrett, P. Barrios, A. Delage, I. Golub, S. Janz, R. McKinnon, P. Poole, and S. Raymond, "Optical switching in InGaAsP waveguides using localized index gradients," Journal of Vacuum Science and Technology A Vacuum, Surfaces, and Films, vol. 22, pp. 796-9, 2004.

[68] S. Ju Bin and R. Killey, "Measurement of absorption coefficient for quantum confined Stark effect InGaAsP/InP MQW and refractive index change," Journal of the Optical Society of Korea, vol. 5, pp. 123-30, 2001.

[69] P. Barrios, P. Poole, M. Buchanan, P. Chow-Chong, I. Golub, C. Lacelle, B. Syrett, S. Abdalla, and S. El Mougy, "InGaAsP digital optical switch on InP," Proceedings of the SPIE The International Society for Optical Engineering, vol. 4833, pp. 735-741, 2002.

[70] S. Ng, S. Abdalla, B. Syrett, P. Barrios, W. R. McKinnon, A. Delage, I. Golub, $\mathrm{S}$. Janz, and J. Lapointe, "Electrical isolation of electrodes with submicron separation in a digital optical switch," Journal of Vacuum Science and Technology A Vacuum, Surfaces, and Films, vol. 24, pp. 807-11, 2006.

[71] M. Sotoodeh, A. H. Khalid, and A. A. Rezazadeh, "Empirical low-field mobility model for III-V compounds applicable in device simulation codes," Journal of Applied Physics, vol. 87, pp. 2890-900, 2000.

[72] J. H. Angenent, M. Erman, J. M. Auger, and R. Gamonal, "Extremely low loss InP/GaInAsP rib waveguides," Electronics Letters, vol. 25, pp. 628-629, 1989. 
[73] G. P. Agrawal and N. K. Dutta, Semiconductor Lasers, 2nd ed. New York: Van Nostrand Reinhold, 1993.

[74] G. Schraud, G. Muller, L. Stoll, and U. Wolff, "Simple measurement of carrier induced refractive-index change in InGaAsP PIN ridge waveguide structures," Electronics Letters, vol. 27, pp. 297-8, 1991.

[75] M. Zegaoui, D. Decoster, J. Harari, J. P. Vilcot, F. Mollot, V. Magnin, and J. Chazelas, "Comparison between carried-induced optical index, loss variations and carrier lifetime in GaInAsP/InP heterostructures for $1.55 \mathrm{mu} \mathrm{m}$ DOS application," Electronics Letters, vol. 41, pp. 613-14, 2005.

[76] F. Urbach, "The Long-Wavelength Edge of Photographic Sensitivity and of the Electronic Absorption of Solids," Physical Review, vol. 92, pp. 1324, 1953.

[77] J. S. Weiner, D. S. Chemla, D. A. B. Miller, H. A. Haus, A. C. Gossard, W. Wiegmann, and C. A. Burrus, "Highly anisotropic optical properties of single quantum well waveguides," Applied Physics Letters, vol. 47, pp. 664-667, 1985.

[78] S. Jong In, M. Yamaguchi, P. Delansay, and M. Kitamura, "Refractive index and loss changes produced by current injection in InGaAs(P)-InGaAsP multiple quantum-well (MQW) waveguides," IEEE Journal of Selected Topics in Quantum Electronics, vol. 1, pp. 408-15, 1995.

[79] U. G. Akano, I. V. Mitchell, F. R. Shepherd, C. J. Miner, and R. Rousina, "Implant-damage isolation of InP and InGaAsP," Journal of Vacuum Science and Technology A Vacuum, Surfaces, and Films, vol. 11, pp. 1016-21, 1993.

[80] S. J. Pearton, "Ion implantation for isolation of III-V semiconductors," Material Science Reports, vol. 4, pp. 313-67, 1990.

[81] K. Blumenstock, J. Theisen, P. Pan, J. Dulak, A. Ticknor, and T. Sandwick, "Shallow trench isolation for ultra-large-scale integrated devices," Journal of Vacuum Science and Technology B Microelectronics and Nanometer Structures, vol. 12 , pp. 54-8, 1994.

[82] H. B. Pogge, "Trench isolation technology," presented at Proceedings of the 1990 Bipolar Circuits and Technology Meeting (Cat. No.90CH2890 2). Minneapolis, MN, USA. IEEE. 1718 Sept. 1990., 1990.

[83] J. A. Cavailles, M. Renaud, J. F. Vinchant, M. Erman, P. Svensson, and L. Thylen, "First digital optical switch based on InP/GaInAsP double heterostructure waveguides," Electronics Letters, vol. 27, pp. 699-700, 1991. 
[84] J. L. Egley and D. Chidambarrao, "Strain effects on device characteristics: implementation in drift-diffusion simulators," Solid State Electronics, vol. 36, pp. 1653-64, 1993.

[85] B. Sermage, J. L. Benchimol, and G. M. Cohen, "Carrier lifetime in p-doped InGaAs and InGaAsP," presented at Conference Proceedings. 1998 International Conference on Indium Phosphide and Related Materials. Tsukuba, Japan. Japan Soc. Appl. Phys. IEEE/Lasers \& Electro Opt. Soc. IEEE Electron Devices Soc. Univ. Tsukuba. IEICE of Japan. Optoelectron. Ind. \& Technol. Dev. Assoc. Res. \& Dev. Assoc. Res. \& Dev. Assoc. Future Electron Devices. 1115 May 1998., 1998.

[86] S. Abdalla, S. Ng, P. Barrios, D. Celo, A. Delage, S. El Mougy, I. Golub, J. J. He, S. Janz, R. McKinnon, P. Poole, S. Raymond, T. J. Smy, and B. Syrett, "Carrier injection-based digital optical switch with reconfigurable output waveguide arms," IEEE Photonics Technology Letters, vol. 16, pp. 1038-40, 2004.

[87] S. Janz, P. Barrios, A. Delage, I. Golub, J. J. He, W. R. McKinnon, P. Poole, S. Abdalla, D. Celo, S. El Mougy, S. Ng, T. J. Smy, and B. Syrett, "Digital optical switch based on fast thermal gradient switching in InGaAsP waveguides," presented at Integrated Photonics Research. USA. Optical Soc. Of America. 16 19 June 2003., 2003.

[88] M. Cada, G. Muller, A. Greil, L. Stoll, and U. Wolff, "Dynamic switching characteristics of a $4 \mathrm{x} 4 \mathrm{InP} / \mathrm{InGaAsP}$ matrix switch," Electronics Letters, vol. 28, pp. 2149-50, 1992.

[89] "Cisco ONS 15540 ESPx Planning Guide," 2004, pp. 5-4.

[90] Z. Sihua, Z. Daqing, Z. Bingna, and L. Wei, "Design and simulation of a polymeric variable optical attenuator," Proceedings of the SPIE The International Society for Optical Engineering, vol. 5279, pp. 101-6, 2004.

[91] Q. Z. Liu, X. S. Jiang, L. S. Yu, Z. F. Guan, P. K. L. Yu, and S. S. Lau, "A novel processing technique to fabricate planar InGaAsP/InP electroabsorption waveguide modulators," Journal of Electronic Materials, vol. 24, pp. 991-7, 1995.

[92] M. Iodice, G. Coppola, R. C. Zaccuri, and I. Rendina, "Waveguide-vanishingbased optical modulator in embedded all-silicon structure," Proceedings of the SPIE The International Society for Optical Engineering, vol. 5730, pp. 114-124, 2006. 
[93] V. I. Tolstikhin, A. Densmore, K. Pimenov, Y. Logvin, W. Fang, S. Laframboise, and S. Grabtchak, "Monolithically integrated optical channel monitor for DWDM transmission systems," Journal of Lightwave Technology, vol. 22, pp. 146-53, 2004.

[94] U. Koren, B. I. Miller, M. G. Young, T. L. Koch, R. M. Jopson, A. H. Gnavok, J. D. Evankow, and M. Chien, "High frequency modulation of strained layer multiple quantum well optical amplifiers," Electronics Letters, vol. 27, pp. 62-4, 1991.

[95] P. Barrios, S. Janz, A. Delage, I. Golub, R. McKinnon, P. Poole, S. Abdalla, D. Celo, S. Ng, T. Smy, and B. Syrett, "Digital optical switches with reconfigurable output waveguide branches in InP," Proceedings of the SPIE The International Society for Optical Engineering, vol. 5622, pp. 751-754, 2004.

[96] S. Ng, S. Abdalla, P. Barrios, A. Delage, I. Golub, S. Janz, R. McKinnon, P. Poole, and B. Syrett, "Low-current optical switching by carrier-injectioninduced reconfigurable waveguiding," Proceedings of the SPIE The International Society for Optical Engineering, vol. 5579, pp. 644-651, 2004.

[97] R. A. Soref and L. R. Schissler, "Crosstalk reduction in optical switching," Applied Optics, vol. 14, pp. 2559-2570, 1975.

[98] T. Arakawa, K. Tada, R. Iino, T. Suzuki, J. H. Noh, N. Haneji, and H. Feng, "Electrorefractive properties of modified five-layer asymmetric coupled quantum well (FACQW)," Microelectronics Journal, vol. 34, pp. 387-90, 2003. 